\title{
AFIP-1 Irradiation Summary Report
}

D. M. Perez

M. A. Lillo

G. S. Chang

G. A. Roth

N. E. Woolstenhulme

D. M. Wachs

May 2011

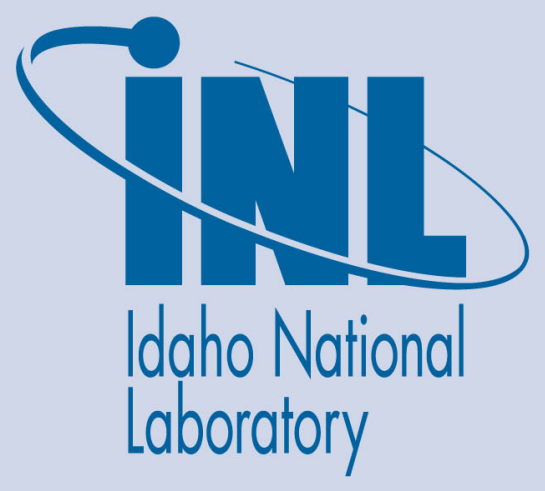

The INL is a U.S. Department of Energy National Laboratory operated by Battelle Energy Alliance 


\section{DISCLAIMER}

This information was prepared as an account of work sponsored by an agency of the U.S. Government. Neither the U.S. Government nor any agency thereof, nor any of their employees, makes any warranty, expressed or implied, or assumes any legal liability or responsibility for the accuracy, completeness, or usefulness, of any information, apparatus, product, or process disclosed, or represents that its use would not infringe privately owned rights. References herein to any specific commercial product, process, or service by trade name, trade mark, manufacturer, or otherwise, does not necessarily constitute or imply its endorsement, recommendation, or favoring by the U.S. Government or any agency thereof. The views and opinions of authors expressed herein do not necessarily state or reflect those of the U.S. Government or any agency thereof. 


\title{
AFIP-1 Irradiation Summary Report
}

\author{
D. M. Perez \\ M. A. Lillo \\ G. S. Chang \\ G. A. Roth
}

N. E. Woolstenhulme

D. M. Wachs

May 2011

Idaho National Laboratory Idaho Falls, Idaho 83415

http://www.inl.gov

\author{
Prepared for the
}

U.S. Department of Energy

Office of National Nuclear Security Administration

Under DOE Idaho Operations Office

Contract DE-AC07-05ID14517 


\section{AFIP-1 Irradiation Summary Report}

INL/EXT-11-22045

May 2011

Approved by:
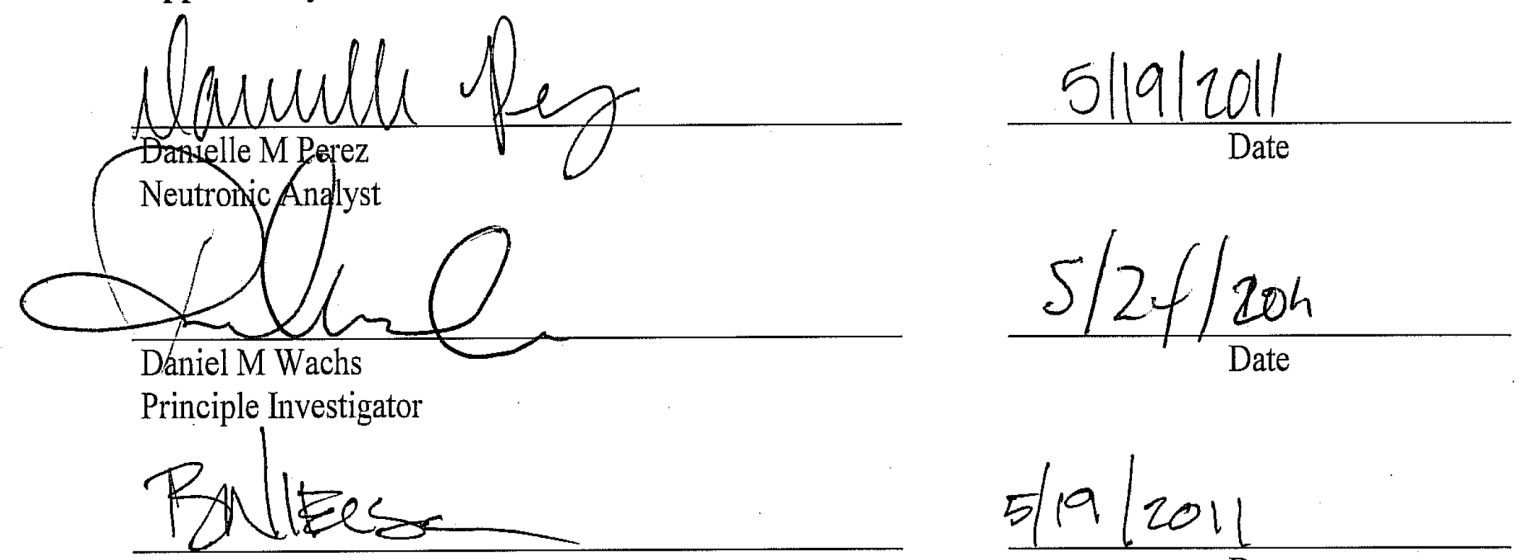

Bruce Nielson

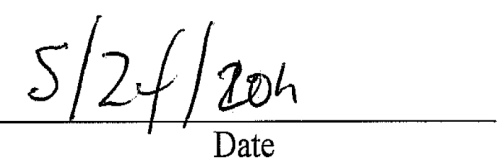

Experiment Manager

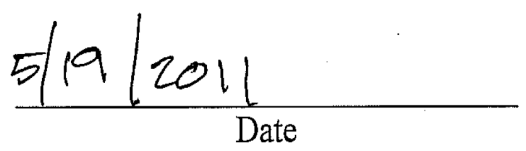




\section{SUMMARY}

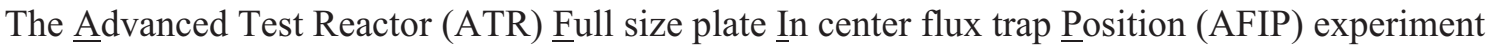
AFIP-1 was designed to demonstrate the performance of second-generation dispersion fuels at a prototypic scale with a length of 21.5 inches $(54.6 \mathrm{~cm})$, width of 2.25 inches $(5.75 \mathrm{~cm})$ and a thickness of 0.050 inch $(0.13 \mathrm{~cm})$. The experiment was fabricated using commercially standard practices at BWX Technology, Inc. (BWXT). The U-7Mo fuel particles were supplied by the Korean Atomic Energy

Research Institute (KAERI) using equipment intended for commercial supply. Two fuel plates were tested that incorporated two different matrix compositions, Al-2Si and Al-4043. ${ }^{1}$

The following report summarizes the life of the AFIP-1 experiment through end of irradiation, including a brief description of the safety analysis, as-run neutronic analysis results, hydraulic testing results, and thermal analysis results 


\section{CONTENTS}

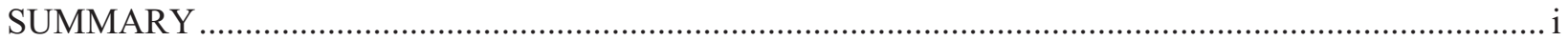

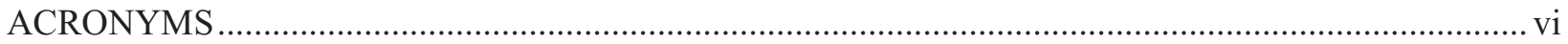

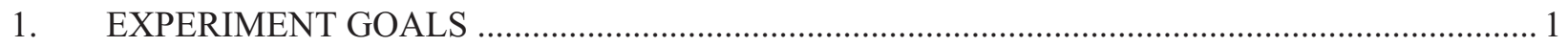

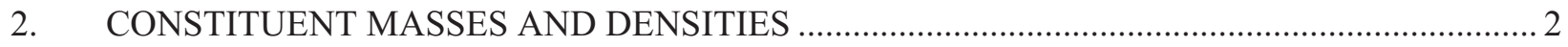

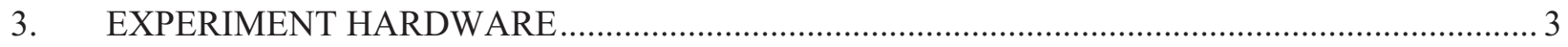

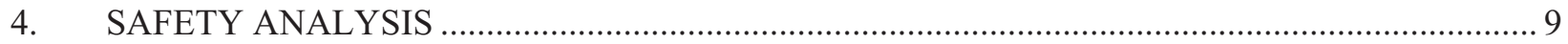

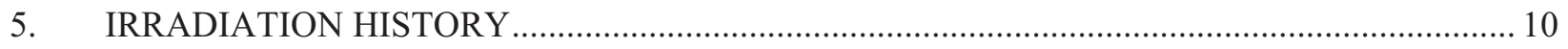

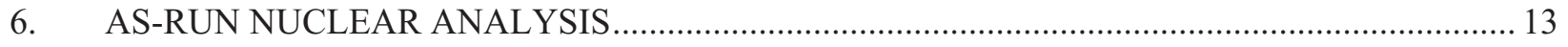

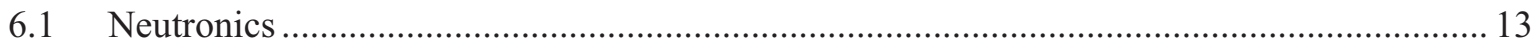

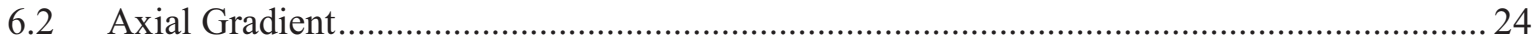

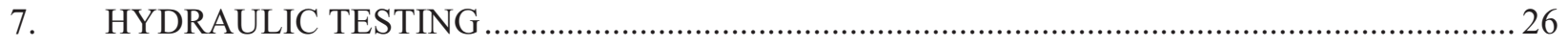

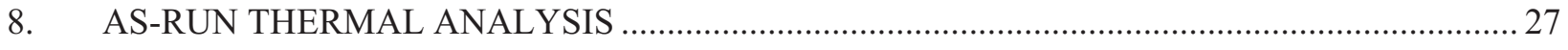

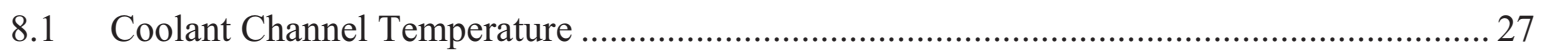

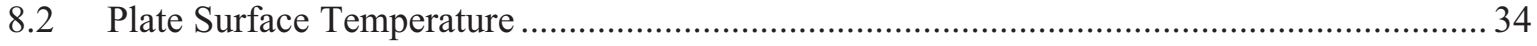

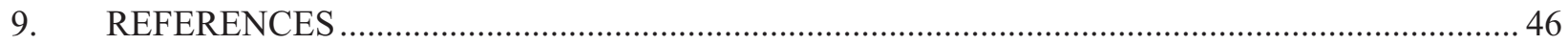

Appendix A Individual Plate Power and Fission Density Plots....................................................... 47

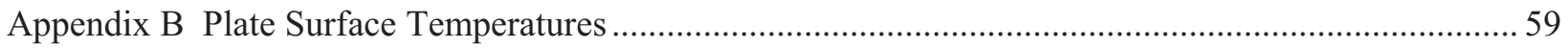

\section{FIGURES}

Figure 1. MCNP model X-Y view of the AFIP-1 test assembly ${ }^{3}$..................................................... 1

Figure 2. DWG 635793 ATR Full Size Plate in Center Flux Trap Position (AFIP) Test Train

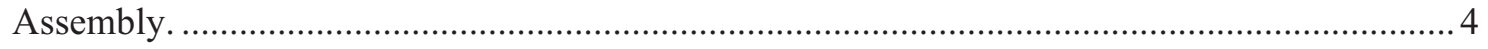

Figure 3. Isometric view of the AFIP-1 Test Train Assembly ............................................................. 5

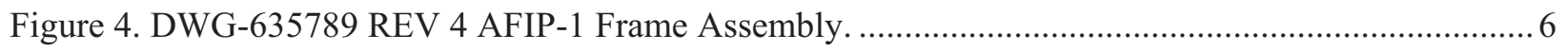

Figure 5. DWG-635788 REV 2 ATR Full Size Plate in Center Flux Trap Position Fuel Plate. ................. 7

Figure 6. Radial Cross Section of the AFIP Test Train Assembly. .......................................................... 8

Figure 7. Hourly lobe power history for ATR Cycle 142B............................................................ 11

Figure 8. Hourly lobe power history for ATR Cycle 143A............................................................. 11

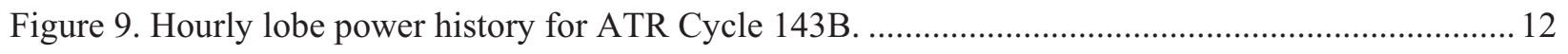

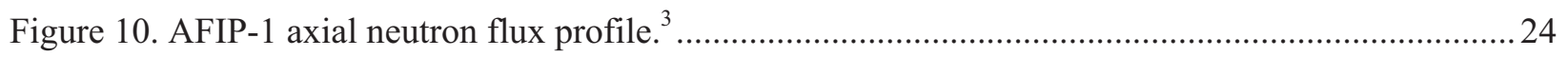


Figure 11. AFIP-1 fission density axial profile ${ }^{3}$.

Figure 12. Coolant channel temperatures as a function of location along the test assembly at BOC 142B (0.0 EFPD). NOTE: AFIP-2 was inserted into plate position A and BOC 142B.

Figure 13. Coolant channel temperatures as a function of location along the test assembly at MOC

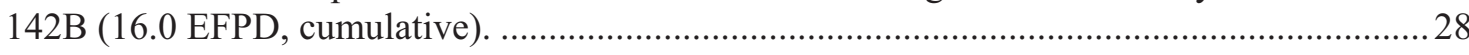

Figure 14. Coolant channel temperatures as a function of location along the test assembly at MOC

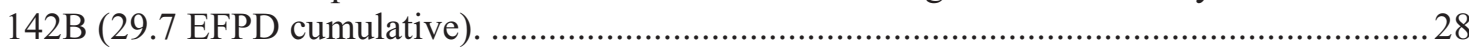

Figure 15. Coolant channel temperatures as a function of location along the test assembly at EOC 142B (52.0 EFPD cumulative).

Figure 16. Coolant channel temperatures as a function of location along the test assembly at BOC 143A (52.0 EFPD cumulative).

Figure 17. Coolant channel temperatures as a function of location along the test assembly at MOC 143A (70.0 EFPD cumulative).

Figure 18. Coolant channel temperatures as a function of location along the test assembly at MOC 143A (90.0 EFPD cumulative).

Figure 19. Coolant channel temperatures as a function of location along the test assembly at EOC 143A (100.9 EFPD cumulative).

Figure 20. Coolant channel temperatures as a function of location along the test assembly at BOC 143B (100.9 EFPD cumulative). NOTE: AFIP-3 was inserted into plate position A and BOC 143B.

Figure 21. Coolant channel temperatures as a function of location along the test assembly at MOC 143B (118.9 EFPD cumulative).

Figure 22. Coolant channel temperatures as a function of location along the test assembly at MOC 143B (138.9 EFPD cumulative). 32

Figure 23. Coolant channel temperatures as a function of location along the test assembly at EOC 143B (158.2 EFPD cumulative).

\section{TABLES}

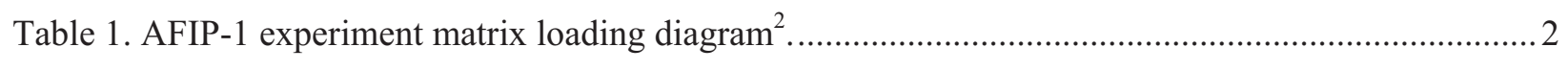

Table 2. AFIP-1 constituent masses ${ }^{4}$........................................................................................ 2

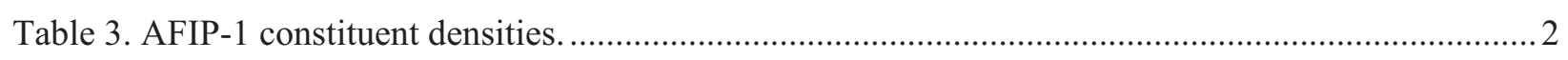

Table 4. AFIP-1 Irradiation Hardware Drawing List....................................................................... 3

Table 5. Summary table of the safety analyses done for the AFIP-1 experiment................................... 9

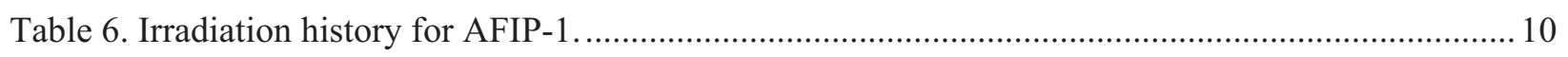

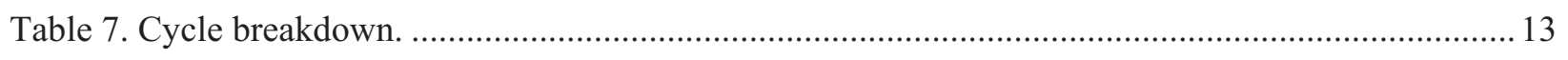

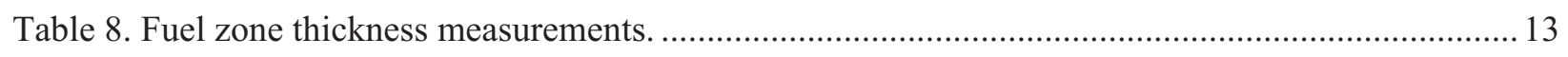

Table 9. End of cycle fission power density, surface heat flux, U-235 depletion and fission density for AFIP-1 experiment.

Table 10. Cycle 142B, MCNP-Calculated heat generation rate and neutron flux for AFIP-1 fuel. 0.0 EFPD (BOC) Center Lobe Power at 25.4 MW. ${ }^{3}$ 
Table 11. Cycle 142B, MCNP-Calculated HGR, Neutron Flux, Depletion, and Fission Density for AFIP-1 fuel, 16.0 EFPD (MOC1), Center Lobe Power at 25.4 MW (16.0 EFPD Cumulative). ${ }^{3}$

Table 12. Cycle 142B, MCNP-Calculated HGR, Neutron Flux, Depletion, and Fission Density for AFIP-1 fuel, 29.7 EFPD (MOC2), Center Lobe Power at 25.4 MW (29.7 EFPD Cumulative). ${ }^{3}$ 16

Table 13.Cycle 142B, MCNP-Calculated HGR, Neutron Flux, Depletion, and Fission Density for AFIP-1 fuel, 52.0 EFPD (EOC), Center Lobe Power at 25.4 MW (52.0 EFPD Cumulative). ${ }^{3}$ 16

Table 14. Cycle 143A, MCNP-Calculated HGR and Neutron Flux for AFIP-1 fuel, 0.0 EFPD (BOC), Center Lobe Power at 24.3 MW. ${ }^{3}$

Table 15. Cycle 143A, MCNP-Calculated HGR, Neutron Flux, Depletion, and Fission Density for AFIP-1 fuel, 18.0 EFPD (MOC1), Center Lobe Power at 24.3 MW (70.0 EFPD Cumulative). ${ }^{3}$

Table 16. Cycle 143A, MCNP-Calculated HGR, Neutron Flux, Depletion, and Fission Density for AFIP-1 fuel, 38.0 EFPD (MOC2), Center Lobe Power at 24.3 MW (90.0 EFPD Cumulative). ${ }^{3}$

Table 17. Cycle 143A, MCNP-Calculated HGR, Neutron Flux, Depletion, and Fission Density for AFIP-1 fuel, 48.9 EFPD (EOC), Center Lobe Power at 24.3 MW (100.9 EFPD Cumulative). ${ }^{3}$

Table 18. Cycle 143B, MCNP-Calculated HGR and Neutron Flux for AFIP-1 fuel, 0.0 EFPD (BOC), Center Lobe Power at 24.9 MW. ${ }^{3}$

Table 19. Cycle 143B, MCNP-Calculated HGR, Neutron Flux, Depletion, and Fission Density for AFIP-1 fuel, 18.0 EFPD (MOC1), Center Lobe Power at 24.9 MW (118.9 EFPD Cumulative). ${ }^{3}$

Table 20. Cycle 143B, MCNP-Calculated HGR, Neutron Flux, Depletion, and Fission Density for AFIP-1 fuel, 38.0 EFPD (MOC2), Center Lobe Power at 24.9 MW (138.9 EFPD Cumulative). ${ }^{3}$

Table 21. Cycle 143B, MCNP-Calculated HGR, Neutron Flux, Depletion, and Fission Density for AFIP-1 fuel, 57.3 EFPD (EOC), Center Lobe Power at 24.9 MW (158.2 EFPD Cumulative). ${ }^{3}$.

Table 22. Corrected fission power density (W/cc) and fission density (fissions/cc) for ATR Cycle 142B.

Table 23. Corrected fission power density (W/cc) and fission density (fissions/cc) for ATR Cycle $143 \mathrm{~A}$. 22

Table 24. Corrected fission power density (W/cc) and fission density (fissions/cc) for ATR Cycle 143B. 23

Table 25. AFIP irradiation vehicle flow conditions for each orifice configuration. ${ }^{6}$.................................26

Table 26. Temperature $\left({ }^{\circ} \mathrm{C}\right)$ map of the east side of plate 1T2 at EOC 142B (52.0 EFPD)...................... 34

Table 27. Temperature $\left({ }^{\circ} \mathrm{C}\right)$ map of the west side of plate $1 \mathrm{~T} 2$ at EOC 142B (52.0 EFPD).................... 35

Table 28. Temperature $\left({ }^{\circ} \mathrm{C}\right)$ map of the east side of plate 1B5 at EOC 142B (52.0 EFPD)..................... 36

Table 29. Temperature $\left({ }^{\circ} \mathrm{C}\right)$ map of the west side of plate $1 \mathrm{~B} 5$ at EOC 142B (52.0 EFPD).................... 37 
Table 30. Temperature $\left({ }^{\circ} \mathrm{C}\right)$ map of the east side of plate $1 \mathrm{~T} 2$ at EOC 143A (100.9 EFPD)................... 38

Table 31. Temperature $\left({ }^{\circ} \mathrm{C}\right)$ map of the west side of plate 1T2 at EOC 143A (100.9 EFPD)................... 39

Table 32. Temperature $\left({ }^{\circ} \mathrm{C}\right)$ map of the east side of plate 1B5 at EOC 143A (100.9 EFPD)..................40

Table 33. Temperature $\left({ }^{\circ} \mathrm{C}\right)$ map of the west side of plate 1B5 at EOC 143A (100.9 EFPD).................. 41

Table 34. Temperature $\left({ }^{\circ} \mathrm{C}\right)$ map of the east side of plate $1 \mathrm{~T} 2$ at EOC 143B (158.2 EFPD)...................42

Table 35. Temperature $\left({ }^{\circ} \mathrm{C}\right)$ map of the west side of plate 1T2 at EOC 143B (158.2 EFPD).................. 43

Table 36. Temperature $\left({ }^{\circ} \mathrm{C}\right)$ map of the east side of plate 1B5 at EOC 143B (158.2 EFPD)...................4 44

Table 37. Temperature $\left({ }^{\circ} \mathrm{C}\right)$ map of the west side of plate $1 \mathrm{~B} 5$ at EOC 143B (158.2 EFPD)..................45 


\section{ACRONYMS}

\begin{tabular}{ll} 
Al & Aluminum \\
ATR & Advanced Test Reactor \\
AFIP & ATR Full-size plate In center flux trap Position \\
BWXT & BWX Technologies, Inc. \\
CFT & Center Flux Trap \\
DAS & Data Acquisition System \\
DNBR & Departure from Nucleate Boiling Ratio \\
ECAR & Engineering Calculations and Analysis Report \\
EFPD & Effective Full Power Days \\
FD & Fuel Development \\
FIR & Flow Instability Ratio \\
GTRI & Global Threat Reduction Initiative \\
KAERI & Korean Atomic Energy Research Institute \\
MCNP & Monte Carlo N-Particle \\
Mo & Molybdenum \\
RERTR & Reduced Enrichment Research and Test Reactor \\
Si & Silicon \\
U & Uranium \\
U-Mo & Uranium-Molybdenum \\
\hline
\end{tabular}




\section{AFIP-1 Irradiation Summary Report \\ 1. EXPERIMENT GOALS}

In support of the Global Threat Reduction Initiative (GTRI) Fuel Development (FD) program (historically known as Reduced Enrichment for Research and Test Reactors (RERTR)), The Advanced Test Reactor (ATR) Full size plate In center flux trap Position (AFIP) experiment AFIP-1 was designed to demonstrate the performance of second-generation dispersion fuels at a prototypic scale with a length of 21.5 inches $(54.6 \mathrm{~cm})$, width of 2.25 inches $(5.75 \mathrm{~cm})$ and a thickness of 0.050 inch $(0.13 \mathrm{~cm})$. The experiment was fabricated using commercially standard practices at BWX Technology, Inc. (BWXT). The U-7Mo fuel particles were supplied by the Korean Atomic Energy Research Institute (KAERI) using equipment intended for commercial supply. Two fuel plates were tested that incorporated two different matrix compositions, Al-2Si and Al-4043. ${ }^{1}$

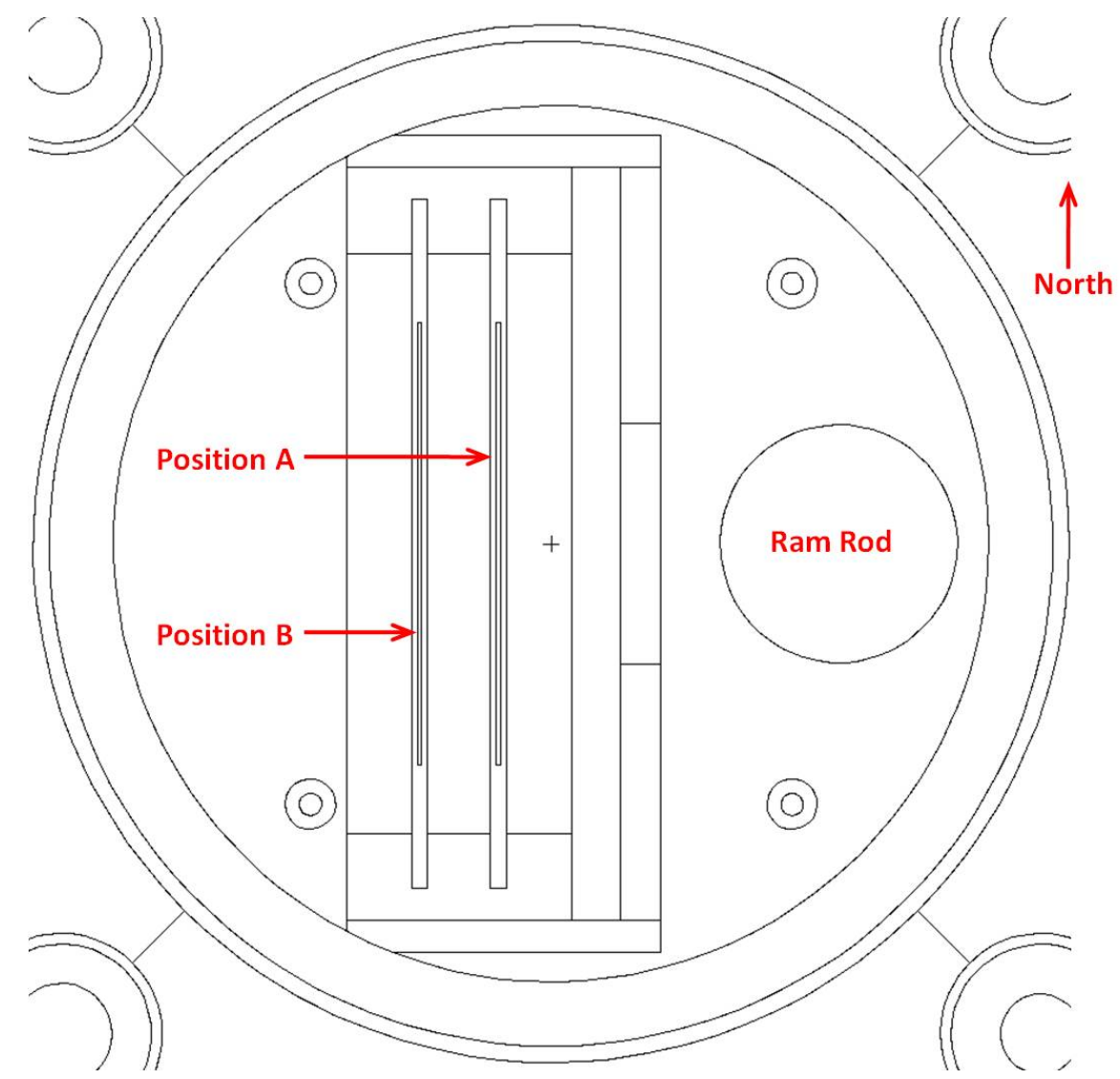

Figure 1. MCNP model X-Y view of the AFIP-1 test assembly ${ }^{3}$.

The AFIP-1 test assembly holds two full length (48 inch) frames, designated as A and B, a ramrod and four flux monitor wires. Frame A is located closest to the ramrod and frame B is on the outer (west) location in the assembly (see Figure 1). Each fuel plate is nominally 22.5 inches in length, 2.205 inches in width and 0.050 inches in thickness. The nominal fuel meat zone is 20.625 inches in length and 1.450 inches in width. The AFIP-1 experiment consisted of two fuel plates that were located in the B position of the test assembly with a plate on the top half of the frame and a plate on the bottom half. The experiment matrix is shown in Table 1. 
Table 1. AFIP-1 experiment matrix loading diagram ${ }^{2}$.

\begin{tabular}{|c|c|c|}
\hline $\begin{array}{c}\text { Assembly } \\
\text { Position }\end{array}$ & Plate ID & B-Position \\
\hline & & U-7Mo \\
Top & $1 \mathrm{~T} 2$ & Al-2Si \\
& & Roll \\
\hline & & U-7Mo \\
Bottom & 1B5 & Al-4043 \\
& & Roll \\
\hline
\end{tabular}

\section{CONSTITUENT MASSES AND DENSITIES}

The constituent masses and densities for plate 1T2 and 1B5 were obtained from the as-built data package ${ }^{4}$ plate summary sheets. Table 2 summarizes the constituent masses for both plates and Table 3 summarizes the constituent densities for both plates.

Table 2. AFIP-1 constituent masses ${ }^{4}$.

\begin{tabular}{|c|c|c|c|c|c|}
\hline $\begin{array}{c}\text { Fuel Plate } \\
\text { Location }\end{array}$ & $\begin{array}{c}\text { Fuel } \\
\text { Plate ID }\end{array}$ & $\begin{array}{c}\text { Total-U } \\
(\mathrm{g})\end{array}$ & $\begin{array}{c}\mathrm{U}-238 \\
(\mathrm{~g})\end{array}$ & $\begin{array}{c}\mathrm{U}-235 \\
(\mathrm{~g})\end{array}$ & $\begin{array}{c}\text { Mo } \\
(\mathrm{g})\end{array}$ \\
\hline Top & $1 \mathrm{~T} 2$ & 101.80 & 81.59 & 20.21 & 7.69 \\
\hline Bottom & $1 \mathrm{~B} 5$ & 102.25 & 81.95 & 20.30 & 7.72 \\
\hline
\end{tabular}

Table 3. AFIP-1 constituent densities.

\begin{tabular}{|c|c|c|c|c|c|c|}
\hline $\begin{array}{c}\text { Fuel Plate } \\
\text { Location }\end{array}$ & $\begin{array}{c}\text { Fuel Plate } \\
\text { ID }\end{array}$ & $\begin{array}{c}\text { Fuel Meat } \\
\text { Volume } \\
(\mathrm{cc})\end{array}$ & $\begin{array}{c}\text { Total-U } \\
(\mathrm{g} / \mathrm{cc})\end{array}$ & $\begin{array}{c}\text { U-238 } \\
(\mathrm{g} / \mathrm{cc})\end{array}$ & $\begin{array}{c}\mathrm{U}-235 \\
(\mathrm{~g} / \mathrm{cc})\end{array}$ & $\begin{array}{c}\text { Mo } \\
(\mathrm{g} / \mathrm{cc})\end{array}$ \\
\hline Top & 1T2 & 11.618 & 8.762 & 7.023 & 1.740 & 0.662 \\
\hline Bottom & 1B5 & 11.618 & 8.801 & 7.054 & 1.747 & 0.664 \\
\hline
\end{tabular}




\section{EXPERIMENT HARDWARE}

The experiment hardware list for AFIP-1 is that used for all of the four-plate AFIP experiments. The drawing numbers and titles are in Table 4.

Table 4. AFIP-1 Irradiation Hardware Drawing List.

\begin{tabular}{cl}
$\begin{array}{c}\text { Drawing } \\
\text { Number }\end{array}$ & \multicolumn{1}{c}{ Drawing Title } \\
\hline 635791 & $\begin{array}{l}\text { ATR Full Size Plate in Center Flux Trap Position (AFIP) Flux Monitor } \\
\text { Wire Holder Assembly Details }\end{array}$ \\
635792 & $\begin{array}{l}\text { ATR Full Size Plate in Center Flux Trap Position (AFIP) Plate Holder } \\
\text { Tube Assembly and Details }\end{array}$ \\
635793 & $\begin{array}{l}\text { ATR Full Size Plate in Center Flux Trap Position (AFIP) Test Train } \\
\text { Assembly } \\
\text { ATR Full Size Plate in Center Flux Trap Position (AFIP) Ramrod and } \\
635790\end{array}$ \\
636544 & $\begin{array}{l}\text { Ram Details } \\
\text { ATR Full Size Plate in Center Flux Trap Position (AFIP) Holder } \\
\text { Retriever Assembly and Details } \\
\text { RERTR ATR Full Size Plate in Center Flux Trap Position Fuel Plate }\end{array}$ \\
635789 & $\begin{array}{l}\text { Frame Assembly and Details } \\
\text { RERTR ATR Full Size Plate in Center Flux Trap Position Fuel Plate }\end{array}$ \\
\hline
\end{tabular}

The AFIP test train assembly as shown in Figure 2 shows the main components of the test assembly, which includes the flux monitor wire holders, the ram and ramrod, the frame assembly and the fuel plates. Figure 3 shows the test train assembly with the retriever attached to the top. The retriever is used to get the test train assembly out of the reactor. The flux monitor wire holders house the flux monitor wires. The ram is used to clamp onto the rails of the fuel plate frame assemblies to minimize vibrations and hold the fuel plate frame assemblies in place. The ramrod is the last component to be inserted; it is used to push the ram against the fuel plate frame assembly. The frame assembly holds the fuel plates into place and is shown in Figure 4. Figure 5 has the specific fuel plate dimensions and nominal fuel foil dimensions. Figure 6 is a radial cross section of the test train assembly and shows the locations of all the components. 


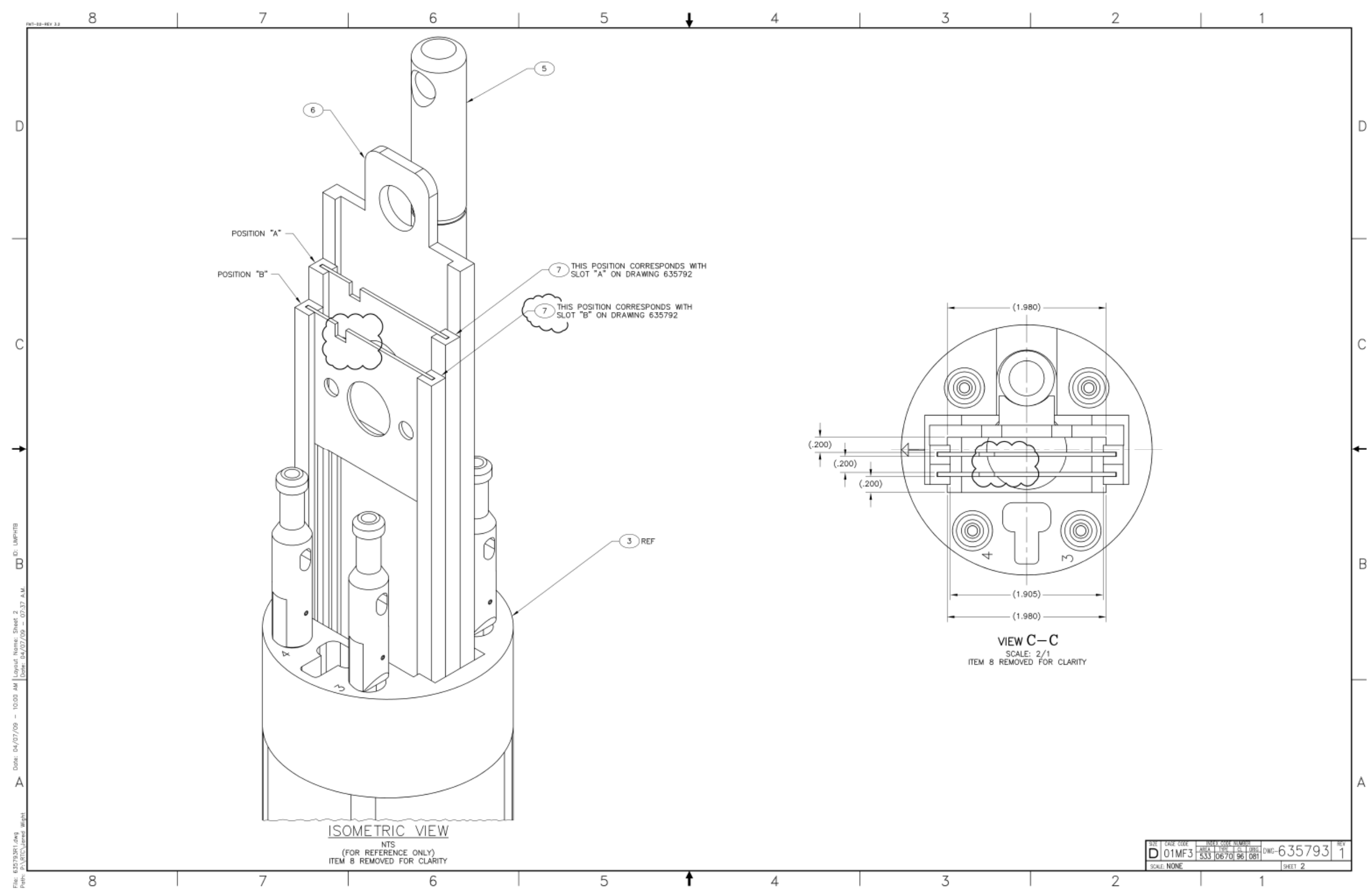

Figure 2. DWG 635793 ATR Full Size Plate in Center Flux Trap Position (AFIP) Test Train Assembly. 


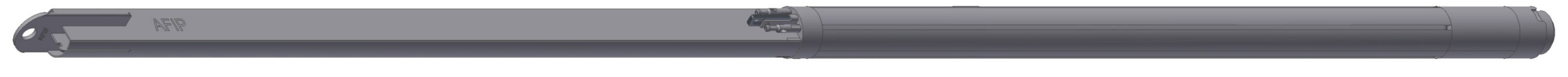

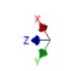

Figure 3. Isometric view of the AFIP-1 Test Train Assembly 


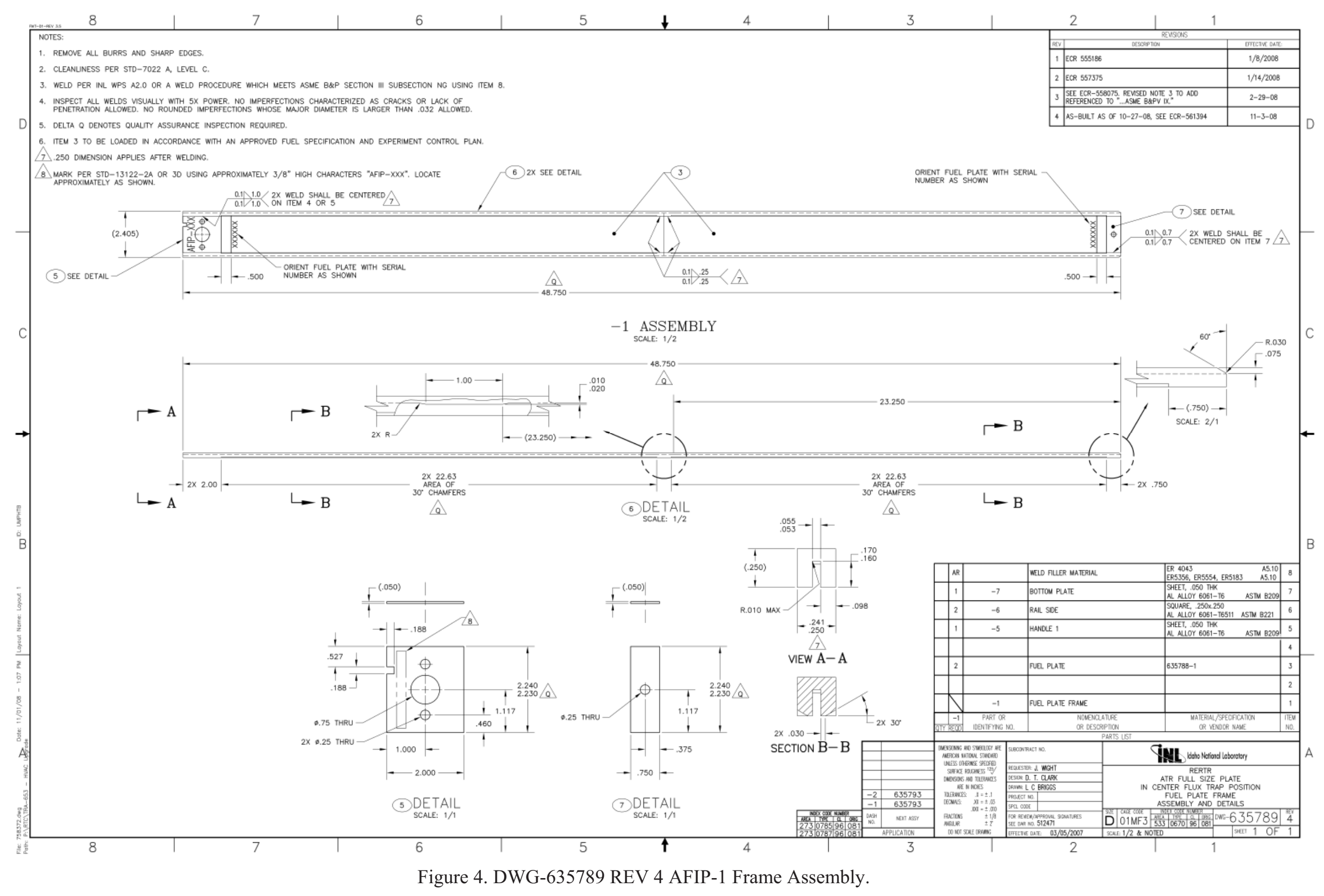




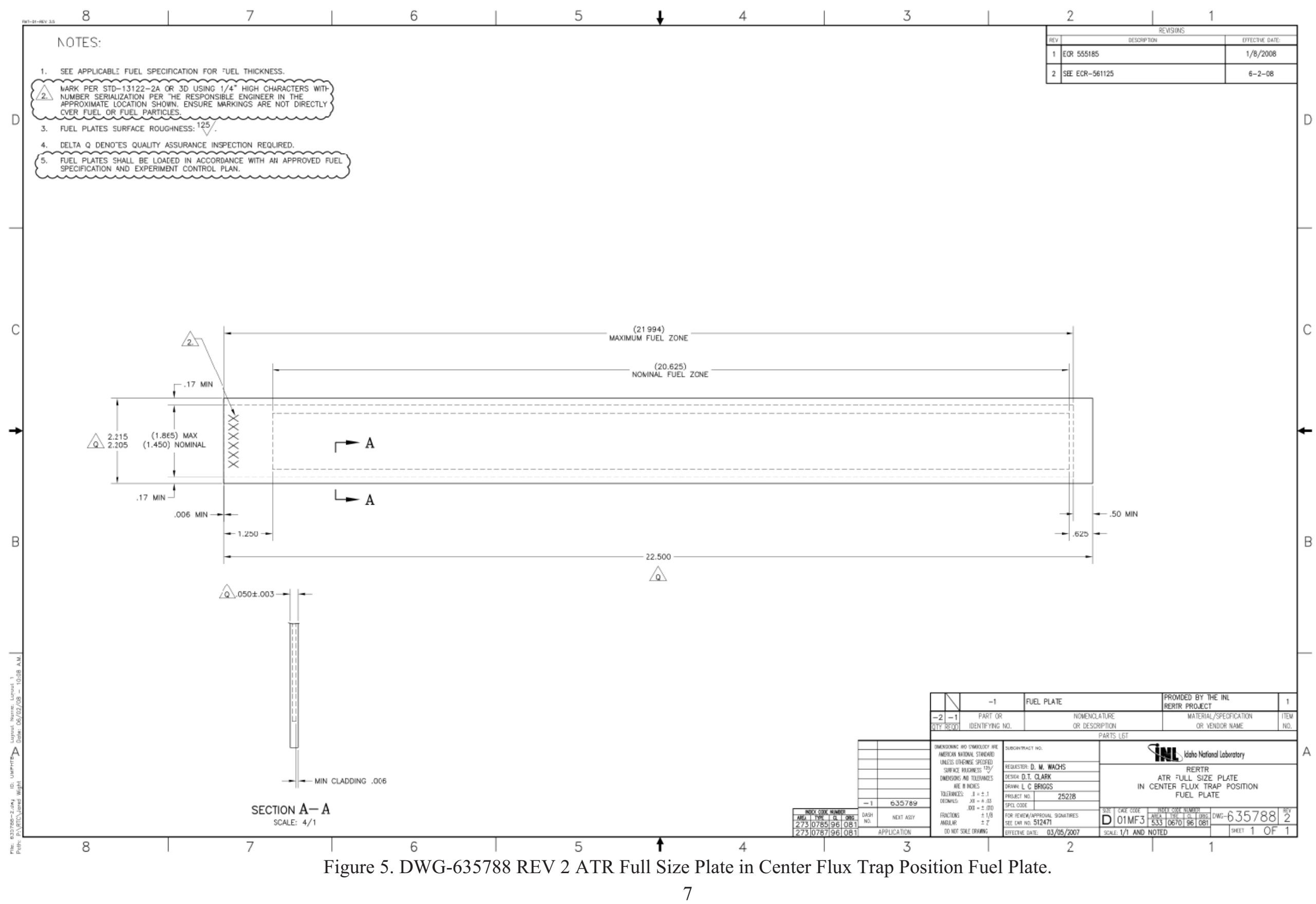




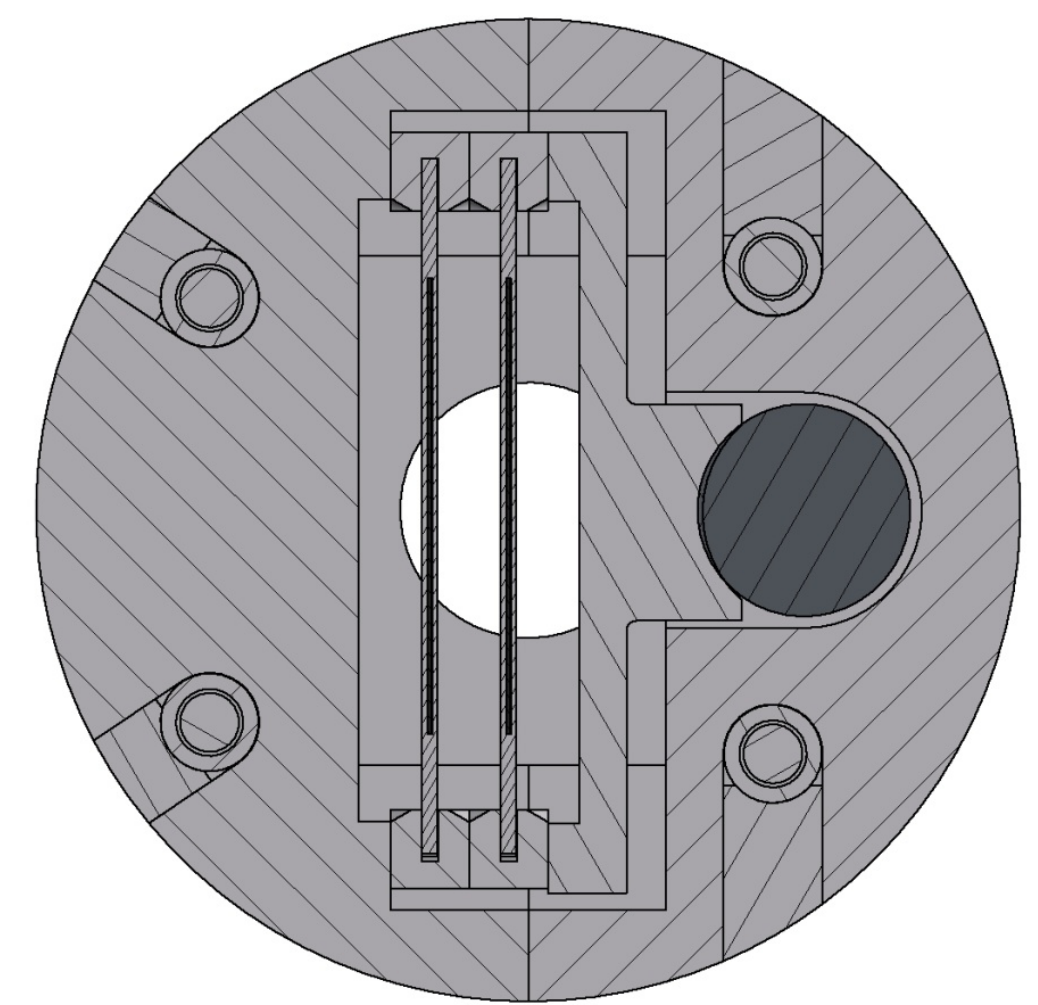

Figure 6. Radial Cross Section of the AFIP Test Train Assembly. 


\section{SAFETY ANALYSIS}

The safety analysis that was performed on the AFIP-1 experiment includes thermal/hydraulic analysis, physics analysis, and structural analysis. The following table (Table 5) summarizes the safety analyses performed on the AFIP-1 experiment.

Table 5. Summary table of the safety analyses done for the AFIP-1 experiment.

\begin{tabular}{|c|l|}
\hline ECAR Number & \multicolumn{1}{c|}{ Description } \\
\hline ECAR-233 & $\begin{array}{l}\text { AFIP Four Plate Bounding Case Neutronics Analysis for the } \\
\text { ATR Center Flux Trap Position }\end{array}$ \\
\hline ECAR-260 & $\begin{array}{l}\text { Results of Four Fuel Plate AFIP Reactivity Measurements in } \\
\text { the ATRC }\end{array}$ \\
\hline ECAR-59, Rev. 2 & $\begin{array}{l}\text { Temperature Reactivity Coefficients for the AFIP } \\
\text { Experiment in the Center Flux Trap of ATR }\end{array}$ \\
\hline ECAE-258 & Thermal Analysis of Four Plate AFIP Experiment \\
\hline EDF-8209 & $\begin{array}{l}\text { AFIP Plate Frame, Test Train, and Backup Analysis } \\
\text { (Structural) }\end{array}$ \\
\hline ECAR-121 & Total Flow Evaluations for AFIP and Backups \\
\hline ECAR-126 & RELAP Analysis for AFIP-2 Flow Restrictor \\
\hline ECAR-432 & $\begin{array}{l}\text { Four Plate AFIP Experiment SIPT }>1 / 2 \text { inch Pump Discharge } \\
\text { Break Reactivity Transient Analysis }\end{array}$ \\
\hline
\end{tabular}




\section{IRRADIATION HISTORY}

The AFIP-1 test assembly was irradiated during cycles 142B, 143A and 143B in the ATR Center Flux Trap (CFT). Cycle 142B ran for 52.0 effective full power days (EFPDs) with a center lobe power of 25.4 MW (total core power of 116.8 MW). Cycle 143A ran for 48.9 EFPDs at a center lobe power of 24.3 MW (total core power of 112.2 MW). Cycle 143B ran for 57.3 EFPDs at a center lobe power of 24.9 MW (total core power of 113.0 MW).

There were two mid-cycle SCRAMs during cycle 142B with durations of 3 days and 2 days from dates 7/6/2008 - 7/9/2008 and 8/5/2008 - 8/7/2008, respectively. There were two mid cycle SCRAMs during cycle 143A with durations of 20 days and 5 days, from dates 10/16/2008 - 11/5/2008 and $11 / 21 / 2008$ - 11/26/2008, respectively. There was one mid-cycle SCRAM during cycle 143B with a duration of 2 days, from dates 1/19/2009-1/21/2009. This information is summarized in Table 6 .

Table 6. Irradiation history for AFIP-1.

\begin{tabular}{|c|c|c|c|c|c|c|c|c|}
\hline $\begin{array}{c}\text { ATR } \\
\text { Cycle }\end{array}$ & $\begin{array}{c}\text { AFIP } \\
\text { Test ID }\end{array}$ & $\begin{array}{c}\text { AFIP } \\
\text { Frames }\end{array}$ & $\begin{array}{c}\text { Dates } \\
\text { Irradiated }\end{array}$ & $\begin{array}{c}\text { Cycle } \\
\text { EFPDs }\end{array}$ & $\begin{array}{c}\text { Mid-Cycle } \\
\text { Scram Decay } \\
\text { Days }\end{array}$ & $\begin{array}{c}\text { Post-Cycle } \\
\text { Decay } \\
\text { Days }\end{array}$ & $\begin{array}{c}\text { Center } \\
\text { Flux Trap } \\
\text { Power } \\
\text { (MW) }\end{array}$ & $\begin{array}{c}\text { Total } \\
\text { Core } \\
\text { Power } \\
\text { (MW) }\end{array}$ \\
\hline 142B & AFIP-1/2 & A,B & $\begin{array}{c}07 / 04 / 2008- \\
08 / 30 / 2008\end{array}$ & 52.0 & 5 & 25 & 25.4 & 116.8 \\
\hline 143A & AFIP-1 & B & $\begin{array}{c}09 / 24 / 2008- \\
12 / 06 / 2008\end{array}$ & 48.9 & 25 & 17 & 24.3 & 112.2 \\
\hline 143B & AFIP-1/3 & A,B & $\begin{array}{c}12 / 23 / 2008- \\
02 / 20 / 2009\end{array}$ & 57.3 & 2 & 21 & 24.9 & 113.0 \\
\hline
\end{tabular}

The power history for each cycle is obtained as an ATR Surveillance Report from the ATR Data Acquisition System (DAS). The plots of each lobe power on an hourly basis are shown in Figure 7, Figure 8 and Figure 9 for cycle 142B, 143A and 143B, respectively. 


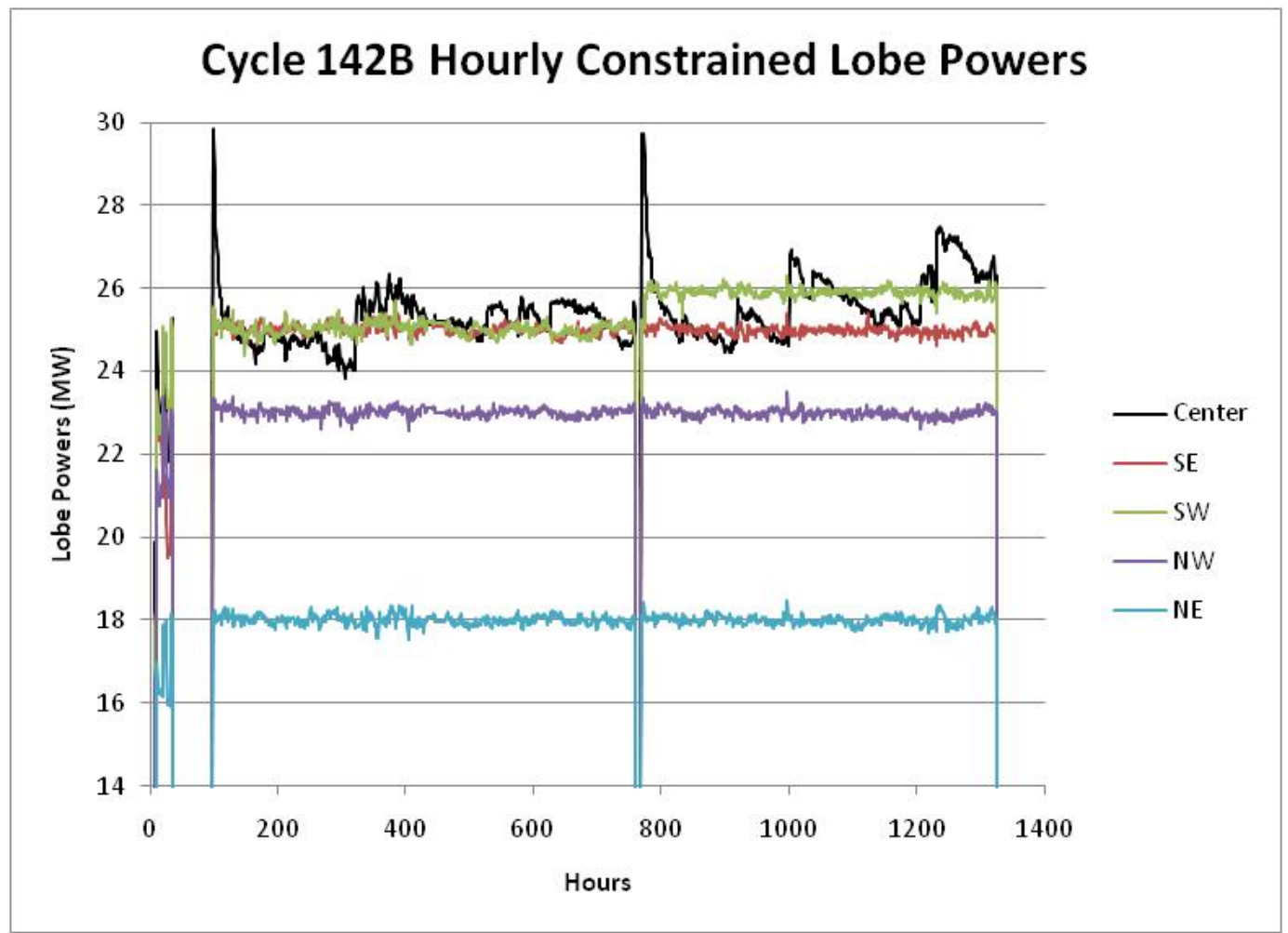

Figure 7. Hourly lobe power history for ATR Cycle 142B.

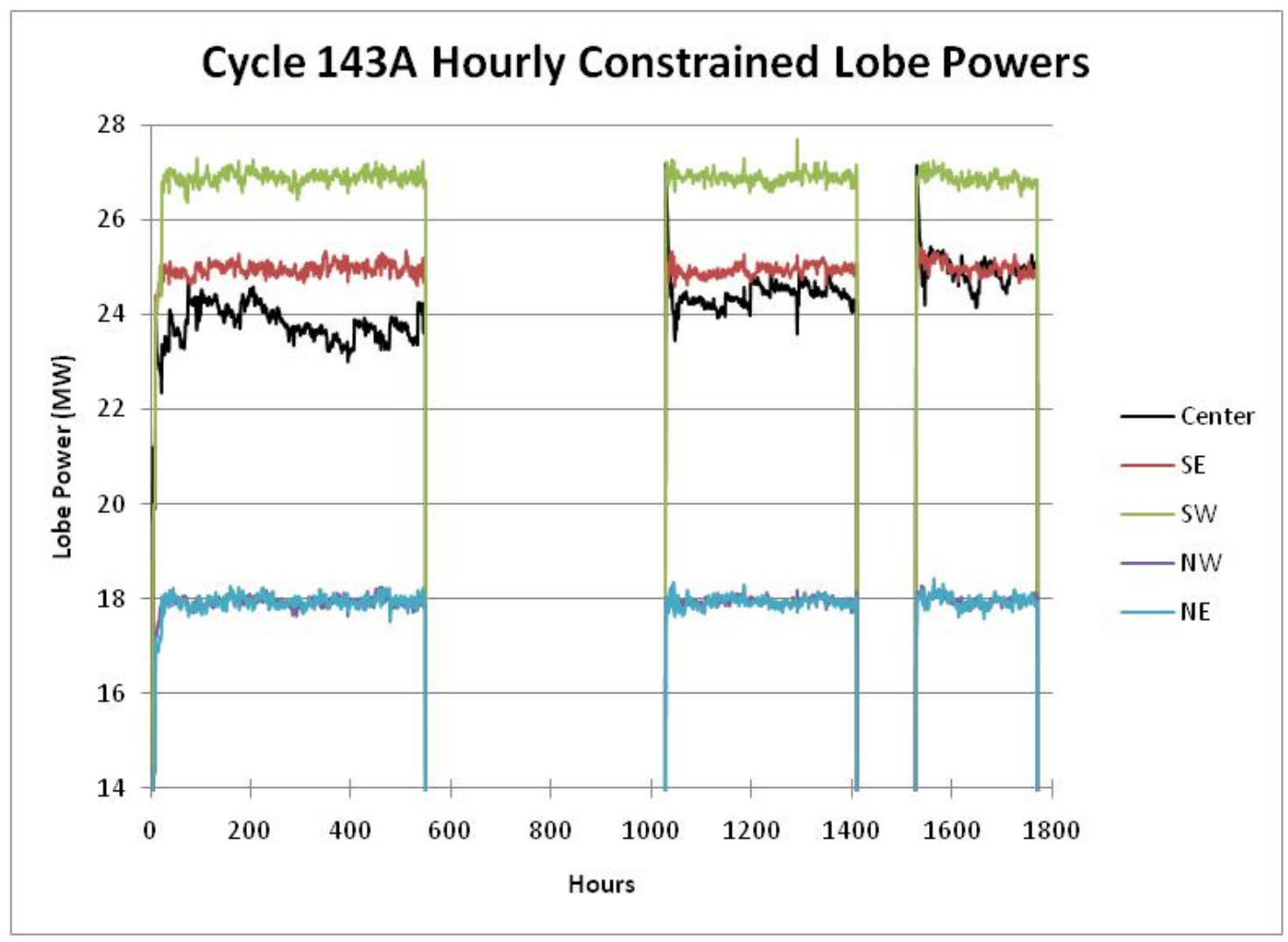

Figure 8. Hourly lobe power history for ATR Cycle 143A. 


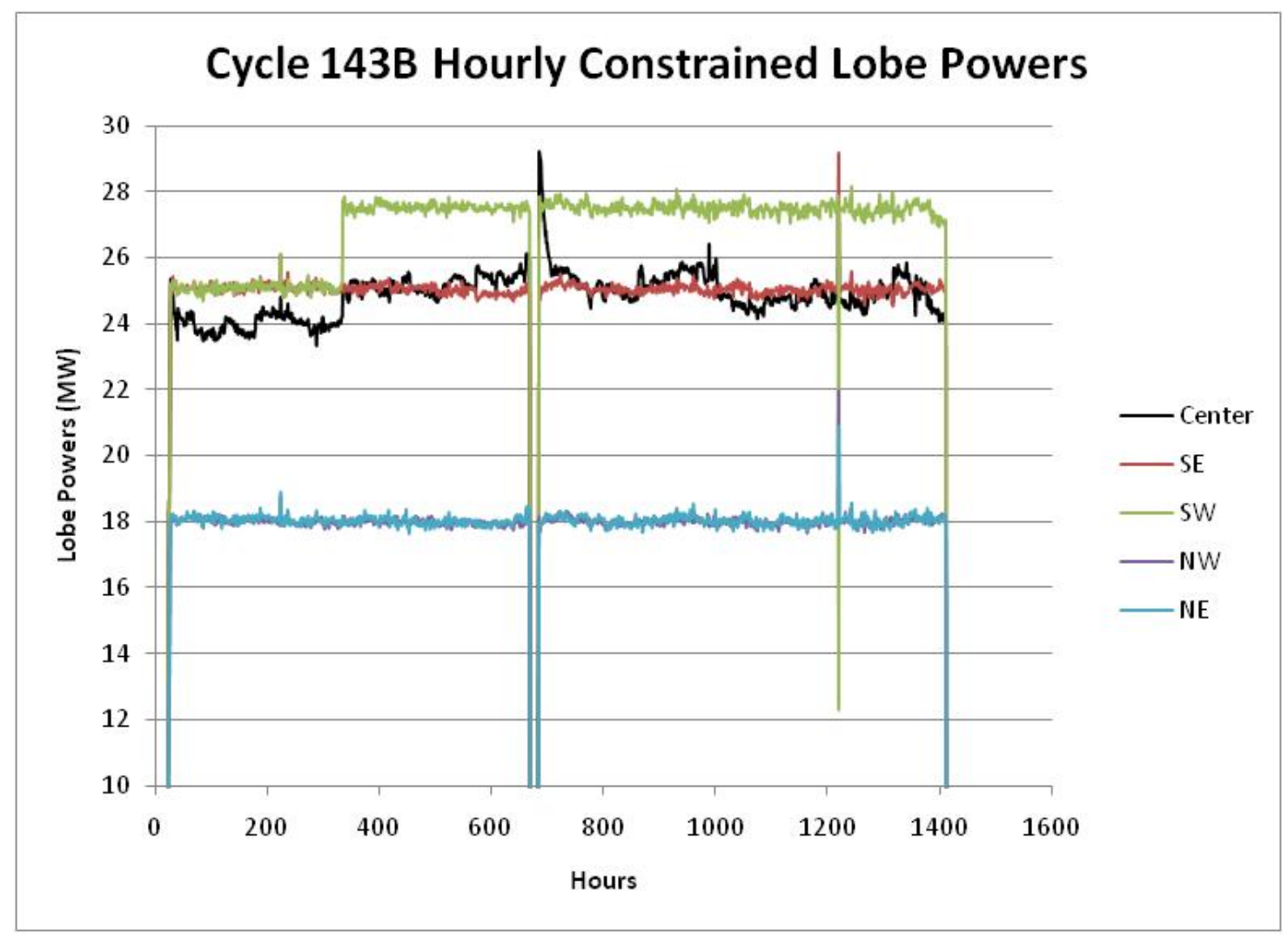

Figure 9. Hourly lobe power history for ATR Cycle 143B. 


\section{AS-RUN NUCLEAR ANALYSIS}

\subsection{Neutronics}

The as-run calculations were performed using the irradiation history in Table 6, the Monte Carlo NParticle (MCNP) code, ORIGEN2.2 and MCWO $^{3}$. The calculated as-run fission heat rates, fission densities, and as-run U-235 burnup results for the fueled plates reported have an uncertainty band $(1 \sigma)$ of $2.5 \%{ }^{3}$. The time intervals used to calculate the average plate power and burnup is shown in Table 7 .

Table 7. Cycle breakdown.

\begin{tabular}{cccc}
\hline $\begin{array}{c}\text { Time } \\
\text { Interval }\end{array}$ & $\begin{array}{c}142 \mathrm{~B} \\
\text { (days) }\end{array}$ & $\begin{array}{c}143 \mathrm{~A} \\
\text { (days) }\end{array}$ & $\begin{array}{c}143 \mathrm{~B} \\
\text { (days) }\end{array}$ \\
\hline BOC & 0.0 & 0.0 & 0.0 \\
MOC 1 & 16.0 & 18.0 & 18.0 \\
MOC 2 & 13.7 & 20.0 & 20.0 \\
EOC & 22.3 & 10.9 & 19.3 \\
\hline Total EFPDs & 52.0 & 48.9 & 57.3 \\
\hline \hline Cumulative & 52.0 & 100.9 & 158.2 \\
\hline
\end{tabular}

The MCNP-calculated neutronic results reported ${ }^{3}$ were calculated using the nominal fuel core thickness reported in the as-built data package ${ }^{4}$ as an "in process" measurement. The actual fuel core thickness measurements were calculated using density measurements and radiography ${ }^{5}$. To correct for the thickness change, the fission density (fissions/cc) and the fission power density (W/cc) were scaled to the thickness measurement ratio shown in Table 8.

Table 8. Fuel zone thickness measurements.

\begin{tabular}{|l|c|c|c|}
\hline Plate ID & $\begin{array}{c}\text { "In-Process" } \\
\text { Average Fuel Foil } \\
\text { Thickness }\end{array}$ & $\begin{array}{c}\text { Measured } \\
\text { Average Fuel Foil } \\
\text { Thickness }^{5} \text { (in) }\end{array}$ & $\begin{array}{c}\text { Thickness } \\
\text { Measurement Ratio }\end{array}$ \\
\hline 1T2 & 0.020 & 0.0221 & 0.905 \\
\hline 1B5 & 0.020 & 0.0227 & 0.881 \\
\hline
\end{tabular}

The MCNP-calculated end of cycle average plate power and burnup for cycles 142B, 143A and 143B are shown in Table 9. The MCNP-calculated plate power and burnup for the time intervals for each cycle are shown in Table 10 through Table 21. The corrected fission density (fissions/cc) and fission power density (W/cc) for each time interval are shown in Table 22 through Table 24 . The heat flux was not scaled since the surface area of the fuel meat was represented correctly in the MCNP model. The plots of the corrected power and fission density as a function of the ATR Cycle time interval are in Appendix A. 
Table 9. End of cycle fission power density, surface heat flux, U-235 depletion and fission density for AFIP-1 experiment.

\begin{tabular}{|c|c|c|c|c|c|c|c|c|c|c|c|c|c|}
\hline \multirow[b]{2}{*}{$\begin{array}{c}\text { Plate } \\
\text { ID }\end{array}$} & \multirow[b]{2}{*}{$\begin{array}{l}\text { MCNP Cell } \\
\text { Elevation }^{(a)} \\
\text { (inches) }^{(1)}\end{array}$} & \multicolumn{4}{|c|}{ EOC 142B } & \multicolumn{4}{|c|}{ EOC 143A } & \multicolumn{4}{|c|}{ EOC 143B } \\
\hline & & $\begin{array}{l}\text { Fission } \\
\text { Power } \\
\text { Density } \\
(\mathrm{W} / \mathrm{cc}) \\
\end{array}$ & $\begin{array}{c}\text { Surface } \\
\text { Heat Flux } \\
\left(\mathrm{W} / \mathrm{cm}^{2}\right)\end{array}$ & $\begin{array}{c}\% \\
\text { Depletion } \\
\text { U-235 } \\
(\%) \\
\end{array}$ & $\begin{array}{c}\text { Fission } \\
\text { Density } \\
\text { (fission/cc) }\end{array}$ & $\begin{array}{l}\text { Fission } \\
\text { Power } \\
\text { Density } \\
(\mathrm{W} / \mathrm{cc})\end{array}$ & $\begin{array}{c}\text { Surface } \\
\text { Heat Flux } \\
\left(\mathrm{W} / \mathrm{cm}^{2}\right)\end{array}$ & $\begin{array}{c}\% \\
\text { Depletion } \\
\text { U-235 } \\
(\%) \\
\end{array}$ & $\begin{array}{c}\text { Fission } \\
\text { Density } \\
\text { (fission/cc) }\end{array}$ & $\begin{array}{l}\text { Fission } \\
\text { Power } \\
\text { Density } \\
(\mathrm{W} / \mathrm{cc})\end{array}$ & $\begin{array}{l}\text { Surface } \\
\text { Heat Flux } \\
\left(\mathrm{W} / \mathrm{cm}^{2}\right)\end{array}$ & $\begin{array}{c}\% \\
\text { Depletion } \\
\text { U-235 } \\
(\%)\end{array}$ & $\begin{array}{c}\text { Fission } \\
\text { Density } \\
\text { (fission/cc) }\end{array}$ \\
\hline \multirow{10}{*}{$1 \mathrm{~T} 2$} & 20.82 & 5020.40 & 127.52 & $19.36 \%$ & $7.58 \mathrm{E}+20$ & 4495.47 & 114.18 & $34.88 \%$ & $1.39 \mathrm{E}+21$ & 3746.04 & 95.15 & $48.61 \%$ & $1.96 \mathrm{E}+21$ \\
\hline & 18.76 & 6209.56 & 157.72 & $24.11 \%$ & $9.55 \mathrm{E}+20$ & 5367.10 & 136.32 & $42.60 \%$ & $1.72 \mathrm{E}+21$ & 4327.45 & 109.92 & $57.76 \%$ & $2.38 \mathrm{E}+21$ \\
\hline & 16.70 & 7385.43 & 187.59 & $29.27 \%$ & $1.18 \mathrm{E}+21$ & 5897.26 & 149.79 & $49.62 \%$ & $2.04 \mathrm{E}+21$ & 4579.03 & 116.31 & $65.46 \%$ & $2.76 \mathrm{E}+21$ \\
\hline & 14.64 & 8293.53 & 210.66 & $33.11 \%$ & $1.35 \mathrm{E}+21$ & 6288.21 & 159.72 & $55.00 \%$ & $2.30 \mathrm{E}+21$ & 4869.80 & 123.69 & $71.27 \%$ & $3.07 \mathrm{E}+21$ \\
\hline & 12.58 & 9033.80 & 229.46 & $36.54 \%$ & $1.51 \mathrm{E}+21$ & 6473.07 & 164.42 & $59.67 \%$ & $2.53 \mathrm{E}+21$ & 4833.14 & 122.76 & $75.64 \%$ & $3.31 \mathrm{E}+21$ \\
\hline & 10.52 & 9456.05 & 240.18 & $38.85 \%$ & $1.61 \mathrm{E}+21$ & 6554.10 & 166.47 & $62.90 \%$ & $2.70 \mathrm{E}+21$ & 4961.85 & 126.03 & $79.00 \%$ & $3.51 \mathrm{E}+21$ \\
\hline & 8.46 & 9825.47 & 249.57 & $40.89 \%$ & $1.71 \mathrm{E}+21$ & 6597.78 & 167.58 & $65.32 \%$ & $2.82 \mathrm{E}+21$ & 4916.25 & 124.87 & $81.06 \%$ & $3.63 \mathrm{E}+21$ \\
\hline & 6.40 & 9967.63 & 253.18 & $42.19 \%$ & $1.77 \mathrm{E}+21$ & 6608.62 & 167.86 & $67.14 \%$ & $2.91 \mathrm{E}+21$ & 4877.30 & 123.88 & $82.67 \%$ & $3.73 \mathrm{E}+21$ \\
\hline & 4.34 & 10177.99 & 258.52 & $42.91 \%$ & $1.81 \mathrm{E}+21$ & 6607.82 & 167.84 & $68.12 \%$ & $2.97 \mathrm{E}+21$ & 4805.66 & 122.06 & $83.52 \%$ & $3.79 \mathrm{E}+21$ \\
\hline & 2.28 & 10365.69 & 263.29 & $44.44 \%$ & $1.88 \mathrm{E}+21$ & 6575.32 & 167.01 & $69.56 \%$ & $3.05 \mathrm{E}+21$ & 4806.01 & 122.07 & $84.75 \%$ & $3.87 \mathrm{E}+21$ \\
\hline \multirow{10}{*}{ 1B5 } & -2.28 & 10382.49 & 263.72 & $404.00 \%$ & $1.91 \mathrm{E}+21$ & 6492.39 & "164.91 & $70.03 \%$ & $3.07 \mathrm{E}+21$ & 47724.65 & " 120.01 & $84.91 \%$ & $3.88 \mathrm{E}+21$ \\
\hline & -4.34 & 10234.02 & 259.94 & $43.54 \%$ & $1.84 \mathrm{E}+21$ & 6584.56 & 167.25 & $68.71 \%$ & $3.00 \mathrm{E}+21$ & 4783.87 & 121.51 & $83.90 \%$ & $3.81 \mathrm{E}+21$ \\
\hline & -6.40 & 10237.54 & 260.03 & $43.41 \%$ & $1.83 \mathrm{E}+21$ & 6618.58 & 168.11 & $68.37 \%$ & $2.99 \mathrm{E}+21$ & 4882.71 & 124.02 & $83.56 \%$ & $3.80 \mathrm{E}+21$ \\
\hline & -8.46 & 10091.34 & 256.32 & $42.53 \%$ & $1.79 \mathrm{E}+21$ & 6586.40 & 167.29 & $67.16 \%$ & $2.92 \mathrm{E}+21$ & 4802.45 & 121.98 & $82.48 \%$ & $3.72 \mathrm{E}+21$ \\
\hline & -10.52 & 9717.88 & 246.83 & $40.96 \%$ & $1.71 \mathrm{E}+21$ & 6553.45 & 166.46 & $65.07 \%$ & $2.81 \mathrm{E}+21$ & 4830.98 & 122.71 & $80.59 \%$ & $3.61 \mathrm{E}+21$ \\
\hline & -12.58 & 9344.22 & 237.34 & $38.99 \%$ & $1.62 \mathrm{E}+21$ & 6462.22 & 164.14 & $62.49 \%$ & $2.67 \mathrm{E}+21$ & 4910.92 & 124.74 & $78.32 \%$ & $3.47 \mathrm{E}+21$ \\
\hline & -14.64 & 8833.71 & 224.38 & $36.00 \%$ & $1.48 \mathrm{E}+21$ & 6368.38 & 161.76 & $58.88 \%$ & $2.49 \mathrm{E}+21$ & 4775.40 & 121.30 & $74.88 \%$ & $3.26 \mathrm{E}+21$ \\
\hline & -16.70 & 8140.28 & 206.76 & $32.75 \%$ & $1.33 \mathrm{E}+21$ & 6015.40 & 152.79 & $54.10 \%$ & $2.26 \mathrm{E}+21$ & 4743.98 & 120.50 & $70.17 \%$ & $3.01 \mathrm{E}+21$ \\
\hline & -18.76 & 7182.94 & 182.45 & $28.51 \%$ & $1.15 \mathrm{E}+21$ & 5606.24 & 142.40 & $48.25 \%$ & $1.98 \mathrm{E}+21$ & 4480.04 & 113.79 & $64.02 \%$ & $2.69 \mathrm{E}+21$ \\
\hline & -20.82 & 6125.34 & 155.58 & $23.87 \%$ & $9.46 \mathrm{E}+20$ & 5062.08 & 128.58 & $41.21 \%$ & $1.66 \mathrm{E}+21$ & 4121.47 & 104.69 & $56.01 \%$ & $2.30 \mathrm{E}+21$ \\
\hline MAX & NA & 10382.49 & 263.72 & $2045.00 \%$ & $1.91 \mathrm{E}+21$ & 6618.58 & 168.11 & $7070.03 \%$ & $3.07 \mathrm{E}+21$ & "4961.85 & 126.03 & $84.91 \%$ & $3.88 \mathrm{E}+21$ \\
\hline MIN & NA & 5020.40 & 127.52 & $19.36 \%$ & $7.58 \mathrm{E}+20$ & 4495.47 & 114.18 & $34.88 \%$ & $1.39 \mathrm{E}+21$ & 3746.04 & 95.15 & $48.61 \%$ & $1.96 \mathrm{E}+21$ \\
\hline AVG & NA & 8801.27 & 223.55 & $36.36 \%$ & $1.51 \mathrm{E}+21$ & 6190.72 & 157.24 & $58.95 \%$ & $2.51 \mathrm{E}+21$ & 4688.95 & 119.10 & $74.43 \%$ & $3.28 \mathrm{E}+21$ \\
\hline
\end{tabular}

(a) Elevations for MCNP cells are reported at cell centroids relative to the modeled core axial midplane at 24.0 inches. 
Table 10. Cycle 142B, MCNP-Calculated heat generation rate and neutron flux for AFIP-1 fuel. 0.0 EFPD (BOC) Center Lobe Power at 25.4 MW. ${ }^{3}$

\begin{tabular}{|c|c|c|c|c|c|}
\hline $\begin{array}{c}\text { Plate } \\
\text { ID }\end{array}$ & Cell & $\begin{array}{l}\text { MCNP Cell } \\
\text { Elevation }^{(a)} \\
\text { (inches) }^{\text {ind }}\end{array}$ & $\begin{array}{c}\text { Fission } \\
\text { Power Density } \\
(\mathrm{W} / \mathrm{cc})\end{array}$ & $\begin{array}{l}\text { Surface } \\
\text { Heat Flux } \\
\left(\mathrm{W} / \mathrm{cm}^{2}\right)\end{array}$ & $\begin{array}{c}\text { Neutron } \\
\text { Flux } \\
\left(\mathrm{n} / \mathrm{cm}^{2} \mathrm{sec}\right) \\
\end{array}$ \\
\hline \multirow{10}{*}{$1 \mathrm{~T} 2$} & A-1 & 20.82 & 4548.21 & 115.52 & $4.58 \mathrm{E}+14$ \\
\hline & A-2 & 18.76 & 5768.14 & 146.51 & $6.03 \mathrm{E}+14$ \\
\hline & A-3 & 16.70 & 7223.07 & 183.47 & $7.54 \mathrm{E}+14$ \\
\hline & A-4 & 14.64 & 8306.64 & 210.99 & $8.63 E+14$ \\
\hline & A-5 & 12.58 & 9461.56 & 240.32 & $9.62 \mathrm{E}+14$ \\
\hline & A-6 & 10.52 & 10300.76 & 261.64 & $1.04 \mathrm{E}+15$ \\
\hline & A-7 & 8.46 & 11031.83 & 280.21 & $1.09 \mathrm{E}+15$ \\
\hline & A-8 & 6.40 & 11568.15 & 293.83 & $1.13 \mathrm{E}+15$ \\
\hline & A-9 & 4.34 & 11833.80 & 300.58 & $1.16 \mathrm{E}+15$ \\
\hline & A-10 & 2.28 & 12430.22 & 315.73 & $1.16 \mathrm{E}+15$ \\
\hline \multirow{13}{*}{$1 \mathrm{~B} 5$} & A-11 & -2.28 & 12845.87 & 326.29 & $1.17 \mathrm{E}+15$ \\
\hline & A-12 & -4.34 & 12176.86 & 309.29 & $1.17 \mathrm{E}+15$ \\
\hline & A-13 & -6.40 & 12034.41 & 305.67 & $1.15 \mathrm{E}+15$ \\
\hline & A-14 & -8.46 & 11798.81 & 299.69 & $1.13 \mathrm{E}+15$ \\
\hline & A-15 & -10.52 & 11276.69 & 286.43 & $1.10 \mathrm{E}+15$ \\
\hline & A-16 & -12.58 & 10480.78 & 266.21 & $1.03 \mathrm{E}+15$ \\
\hline & A-17 & -14.64 & 9454.55 & 240.15 & $9.46 \mathrm{E}+14$ \\
\hline & A-18 & -16.70 & 8348.28 & 212.05 & $8.53 E+14$ \\
\hline & A-19 & -18.76 & 7137.12 & 181.28 & $7.26 \mathrm{E}+14$ \\
\hline & A-20 & -20.82 & 5750.19 & 146.05 & $5.80 \mathrm{E}+14$ \\
\hline & MAX & NA & 12845.87 & 326.29 & $1.17 \mathrm{E}+15$ \\
\hline & MIN & NA & 4548.21 & 115.52 & $4.58 \mathrm{E}+14$ \\
\hline & AVG & NA & 9688.80 & 246.10 & $9.54 \mathrm{E}+14$ \\
\hline
\end{tabular}

(a) Elevations for MCNP cells are reported at cell centroids relative to the modeled core axial midplane at 24.0 inches.

Table 11. Cycle 142B, MCNP-Calculated HGR, Neutron Flux, Depletion, and Fission Density for AFIP-1 fuel, 16.0 EFPD (MOC1), Center Lobe Power at 25.4 MW (16.0 EFPD Cumulative). ${ }^{3}$

\begin{tabular}{|c|c|c|c|c|c|c|c|}
\hline $\begin{array}{c}\text { Plate } \\
\text { ID }\end{array}$ & Cell & $\begin{array}{c}\text { MCNP Cell } \\
\text { Elevation } \\
\text { (inches) }\end{array}$ & $\begin{array}{c}\text { Fission } \\
\text { Power Density } \\
\text { (W/cc) }\end{array}$ & $\begin{array}{c}\text { Surface } \\
\text { Heat Flux } \\
\left(\mathrm{W} / \mathrm{cm}^{2}\right)\end{array}$ & $\begin{array}{c}\text { Neutron } \\
\text { Flux } \\
\left(\mathrm{n} / \mathrm{cm}^{2} \text { sec) }\right.\end{array}$ & $\begin{array}{c}\text { \% Depletion } \\
\text { U-235 } \\
(\%)\end{array}$ & $\begin{array}{c}\text { Fission } \\
\text { Density } \\
\text { (fissions/cc) }\end{array}$ \\
\hline & A-1 & 20.82 & 4558.53 & 115.79 & $4.79 \mathrm{E}+14$ & $5.61 \%$ & $2.15 \mathrm{E}+20$ \\
& A-2 & 18.76 & 5785.33 & 146.95 & $6.23 \mathrm{E}+14$ & $7.07 \%$ & $2.72 \mathrm{E}+20$ \\
& A-3 & 16.70 & 7142.45 & 181.42 & $7.67 \mathrm{E}+14$ & $8.77 \%$ & $3.41 \mathrm{E}+20$ \\
& A-4 & 14.64 & 8216.92 & 208.71 & $8.77 \mathrm{E}+14$ & $10.03 \%$ & $3.92 \mathrm{E}+20$ \\
& A-5 & 12.58 & 9096.70 & 231.06 & $9.70 \mathrm{E}+14$ & $11.33 \%$ & $4.47 \mathrm{E}+20$ \\
& A-6 & 10.52 & 9756.12 & 247.81 & $1.04 \mathrm{E}+15$ & $12.27 \%$ & $4.86 \mathrm{E}+20$ \\
& A-7 & 8.46 & 10395.30 & 264.04 & $1.09 \mathrm{E}+15$ & $13.08 \%$ & $5.21 \mathrm{E}+20$ \\
& A-8 & 6.40 & 10805.19 & 274.45 & $1.11 \mathrm{E}+15$ & $13.66 \%$ & $5.46 \mathrm{E}+20$ \\
& A-9 & 4.34 & 10906.63 & 277.03 & $1.13 \mathrm{E}+15$ & $13.95 \%$ & $5.58 \mathrm{E}+20$ \\
& A-10 & 2.28 & 11401.44 & 289.60 & $1.14 \mathrm{E}+15$ & $14.58 \%$ & $5.87 \mathrm{E}+20$ \\
\hline \hline & A-11 & -2.28 & 11425.36 & 290.20 & $1.14 \mathrm{E}+15$ & $15.03 \%$ & $6.06 \mathrm{E}+20$ \\
& A-12 & -4.34 & 11076.80 & 281.35 & $1.13 \mathrm{E}+15$ & $14.31 \%$ & $5.75 \mathrm{E}+20$ \\
& A-13 & -6.40 & 11050.40 & 280.68 & $1.13 \mathrm{E}+15$ & $14.15 \%$ & $5.68 \mathrm{E}+20$ \\
& A-14 & -8.46 & 10682.59 & 271.34 & $1.10 \mathrm{E}+15$ & $13.89 \%$ & $5.57 \mathrm{E}+20$ \\
& A-15 & -10.52 & 10338.80 & 262.61 & $1.06 \mathrm{E}+15$ & $13.32 \%$ & $5.32 \mathrm{E}+20$ \\
& A-16 & -12.58 & 9808.05 & 249.12 & $1.02 \mathrm{E}+15$ & $12.47 \%$ & $4.95 \mathrm{E}+20$ \\
& A-17 & -14.64 & 8868.18 & 225.25 & $9.41 \mathrm{E}+14$ & $11.31 \%$ & $4.46 \mathrm{E}+20$ \\
& A-18 & -16.70 & 7987.86 & 202.89 & $8.58 \mathrm{E}+14$ & $10.07 \%$ & $3.94 \mathrm{E}+20$ \\
& A-19 & -18.76 & 6874.88 & 174.62 & $7.29 \mathrm{E}+14$ & $8.66 \%$ & $3.37 \mathrm{E}+20$ \\
& A-20 & -20.82 & 5723.37 & 145.37 & $5.87 \mathrm{E}+14$ & $7.04 \%$ & $2.71 \mathrm{E}+20$ \\
\hline \hline & MAX & NA & 11425.36 & 290.20 & $1.14 \mathrm{E}+15$ & $15.03 \%$ & $6.06 \mathrm{E}+20$ \\
\hline & MIN & NA & 4558.53 & 115.79 & $4.79 \mathrm{E}+14$ & $5.61 \%$ & $2.15 \mathrm{E}+20$ \\
\hline & AVG & NA & 9095.04 & 231.01 & $9.46 \mathrm{E}+14$ & $11.53 \%$ & $4.57 \mathrm{E}+20$ \\
\hline
\end{tabular}

(a) Elevations for MCNP cells are reported at cell centroids relative to the modeled core axial midplane at 24.0 inches. 
Table 12. Cycle 142B, MCNP-Calculated HGR, Neutron Flux, Depletion, and Fission Density for AFIP-1 fuel, 29.7 EFPD (MOC2), Center Lobe Power at 25.4 MW (29.7 EFPD Cumulative). ${ }^{3}$

\begin{tabular}{|c|c|c|c|c|c|c|c|}
\hline $\begin{array}{c}\text { Plate } \\
\text { ID }\end{array}$ & Cell & $\begin{array}{c}\text { MCNP Cell } \\
\text { Elevation } \\
\text { (inches) }\end{array}$ & $\begin{array}{c}\text { Fission } \\
\text { Power Density } \\
(\mathrm{W} / \mathrm{cc})\end{array}$ & $\begin{array}{c}\text { Surface } \\
\text { Heat Flux } \\
\left(\mathrm{W} / \mathrm{cm}^{2}\right)\end{array}$ & $\begin{array}{c}\text { Neutron } \\
\text { Flux } \\
\left(\mathrm{n} / \mathrm{cm}^{2} \mathrm{sec}\right)\end{array}$ & $\begin{array}{c}\% \text { Depletion } \\
\text { U-235 } \\
(\%)\end{array}$ & $\begin{array}{c}\text { Fission } \\
\text { Density } \\
\text { (fissions/cc) }\end{array}$ \\
\hline & A-1 & 20.82 & 5323.11 & 135.21 & $5.30 \mathrm{E}+14$ & $10.41 \%$ & $3.99 \mathrm{E}+20$ \\
& A-2 & 18.76 & 6658.21 & 169.12 & $6.91 \mathrm{E}+14$ & $13.10 \%$ & $5.07 \mathrm{E}+20$ \\
& A-3 & 16.70 & 8150.08 & 207.01 & $8.44 \mathrm{E}+14$ & $16.13 \%$ & $6.30 \mathrm{E}+20$ \\
& A-4 & 14.64 & 9290.40 & 235.98 & $9.60 \mathrm{E}+14$ & $18.39 \%$ & $7.25 \mathrm{E}+20$ \\
& A-5 & 12.58 & 10281.12 & 261.14 & $1.06 \mathrm{E}+15$ & $20.52 \%$ & $8.15 \mathrm{E}+20$ \\
& A-6 & 10.52 & 10880.57 & 276.37 & $1.12 \mathrm{E}+15$ & $22.07 \%$ & $8.81 \mathrm{E}+20$ \\
& A-7 & 8.46 & 11419.25 & 290.05 & $1.18 \mathrm{E}+15$ & $23.46 \%$ & $9.42 \mathrm{E}+20$ \\
& A-8 & 6.40 & 11692.42 & 296.99 & $1.20 \mathrm{E}+15$ & $24.43 \%$ & $9.83 \mathrm{E}+20$ \\
& A-9 & 4.34 & 11984.91 & 304.42 & $1.22 \mathrm{E}+15$ & $24.79 \%$ & $1.00 \mathrm{E}+21$ \\
& A-10 & 2.28 & 12369.96 & 314.20 & $1.23 \mathrm{E}+15$ & $25.89 \%$ & $1.05 \mathrm{E}+21$ \\
\hline \hline & A-11 & -2.28 & 12460.40 & 316.49 & $1.23 \mathrm{E}+15$ & $26.36 \%$ & $1.07 \mathrm{E}+21$ \\
& A-12 & -4.34 & 12093.30 & 307.17 & $1.22 \mathrm{E}+15$ & $25.30 \%$ & $1.02 \mathrm{E}+21$ \\
& A-13 & -6.40 & 12117.06 & 307.77 & $1.22 \mathrm{E}+15$ & $25.15 \%$ & $1.02 \mathrm{E}+21$ \\
& A-14 & -8.46 & 11867.27 & 301.43 & $1.20 \mathrm{E}+15$ & $24.56 \%$ & $9.89 \mathrm{E}+20$ \\
1B5 & A-15 & -10.52 & 11333.66 & 287.88 & $1.16 \mathrm{E}+15$ & $23.67 \%$ & $9.51 \mathrm{E}+20$ \\
& A-16 & -12.58 & 10785.64 & 273.96 & $1.11 \mathrm{E}+15$ & $22.34 \%$ & $8.92 \mathrm{E}+20$ \\
& A-17 & -14.64 & 10010.53 & 254.27 & $1.03 \mathrm{E}+15$ & $20.32 \%$ & $8.05 \mathrm{E}+20$ \\
& A-18 & -16.70 & 9143.13 & 232.24 & $9.29 \mathrm{E}+14$ & $18.21 \%$ & $7.17 \mathrm{E}+20$ \\
& A-19 & -18.76 & 7871.58 & 199.94 & $8.01 \mathrm{E}+14$ & $15.77 \%$ & $6.15 \mathrm{E}+20$ \\
& A-20 & -20.82 & 6568.68 & 166.84 & $6.57 \mathrm{E}+14$ & $13.01 \%$ & $5.03 \mathrm{E}+20$ \\
\hline \hline & MAX & NA & 12460.40 & 316.49 & $1.23 \mathrm{E}+15$ & $26.36 \%$ & $1.07 \mathrm{E}+21$ \\
\hline & MIN & NA & 5323.11 & 135.21 & $5.30 \mathrm{E}+14$ & $10.41 \%$ & $3.99 \mathrm{E}+20$ \\
\hline & AVG & NA & 10115.06 & 256.92 & $1.03 \mathrm{E}+15$ & $20.69 \%$ & $8.25 \mathrm{E}+20$ \\
\hline
\end{tabular}

(a) Elevations for MCNP cells are reported at cell centroids relative to the modeled core axial midplane at 24.0 inches.

Table 13.Cycle 142B, MCNP-Calculated HGR, Neutron Flux, Depletion, and Fission Density for AFIP-1 fuel, 52.0 EFPD (EOC), Center Lobe Power at 25.4 MW (52.0 EFPD Cumulative). ${ }^{3}$

\begin{tabular}{|c|c|c|c|c|c|c|c|}
\hline $\begin{array}{l}\text { Plate } \\
\text { ID }\end{array}$ & Cell & $\begin{array}{l}\text { MCNP Cell } \\
\text { Elevation }^{(a)} \\
\text { (inches) }^{\text {inches }}\end{array}$ & $\begin{array}{c}\text { Fission } \\
\text { Power Density } \\
(\mathrm{W} / \mathrm{cc})\end{array}$ & $\begin{array}{l}\text { Surface } \\
\text { Heat Flux } \\
\left(\mathrm{W} / \mathrm{cm}^{2}\right)\end{array}$ & $\begin{array}{c}\text { Neutron } \\
\text { Flux } \\
\left(\mathrm{n} / \mathrm{cm}^{2} \mathrm{sec}\right)\end{array}$ & $\begin{array}{c}\% \text { Depletion } \\
\text { U-235 } \\
(\%)\end{array}$ & $\begin{array}{c}\text { Fission } \\
\text { Density } \\
\text { (fissions/cc) }\end{array}$ \\
\hline \multirow{10}{*}{$1 \mathrm{~T} 2$} & A-1 & 20.82 & 5020.40 & 127.52 & $5.27 \mathrm{E}+14$ & $19.36 \%$ & $7.58 \mathrm{E}+20$ \\
\hline & A-2 & 18.76 & 6209.56 & 157.72 & $6.87 \mathrm{E}+14$ & $24.11 \%$ & $9.55 \mathrm{E}+20$ \\
\hline & A-3 & 16.70 & 7385.43 & 187.59 & $8.39 \mathrm{E}+14$ & $29.27 \%$ & $1.18 \mathrm{E}+21$ \\
\hline & A-4 & 14.64 & 8293.53 & 210.66 & $9.52 \mathrm{E}+14$ & $33.11 \%$ & $1.35 \mathrm{E}+21$ \\
\hline & A-5 & 12.58 & 9033.80 & 229.46 & $1.05 \mathrm{E}+15$ & $36.54 \%$ & $1.51 \mathrm{E}+21$ \\
\hline & A-6 & 10.52 & 9456.05 & 240.18 & $1.12 \mathrm{E}+15$ & $38.85 \%$ & $1.61 \mathrm{E}+21$ \\
\hline & A-7 & 8.46 & 9825.47 & 249.57 & $1.17 \mathrm{E}+15$ & $40.89 \%$ & $1.71 \mathrm{E}+21$ \\
\hline & A-8 & 6.40 & 9967.63 & 253.18 & $1.19 \mathrm{E}+15$ & $42.19 \%$ & $1.77 \mathrm{E}+21$ \\
\hline & A-9 & 4.34 & 10177.99 & 258.52 & $1.21 \mathrm{E}+15$ & $42.91 \%$ & $1.81 \mathrm{E}+21$ \\
\hline & A-10 & 2.28 & 10365.69 & 263.29 & $1.22 \mathrm{E}+15$ & $44.44 \%$ & $1.88 \mathrm{E}+21$ \\
\hline \multirow{13}{*}{ 1B5 } & A-11 & -2.28 & 10382.49 & 263.72 & $1.22 \mathrm{E}+15$ & $45.00 \%$ & $1.91 \mathrm{E}+21$ \\
\hline & A-12 & -4.34 & 10234.02 & 259.94 & $1.21 \mathrm{E}+15$ & $43.54 \%$ & $1.84 \mathrm{E}+21$ \\
\hline & A-13 & -6.40 & 10237.54 & 260.03 & $1.20 \mathrm{E}+15$ & $43.41 \%$ & $1.83 \mathrm{E}+21$ \\
\hline & A-14 & -8.46 & 10091.34 & 256.32 & $1.19 \mathrm{E}+15$ & $42.53 \%$ & $1.79 \mathrm{E}+21$ \\
\hline & A-15 & -10.52 & 9717.88 & 246.83 & $1.15 \mathrm{E}+15$ & $40.96 \%$ & $1.71 \mathrm{E}+21$ \\
\hline & A-16 & -12.58 & 9344.22 & 237.34 & $1.10 \mathrm{E}+15$ & $38.99 \%$ & $1.62 \mathrm{E}+21$ \\
\hline & A-17 & -14.64 & 8833.71 & 224.38 & $1.02 \mathrm{E}+15$ & $36.00 \%$ & $1.48 \mathrm{E}+21$ \\
\hline & A-18 & -16.70 & 8140.28 & 206.76 & $9.21 \mathrm{E}+14$ & $32.75 \%$ & $1.33 \mathrm{E}+21$ \\
\hline & A-19 & -18.76 & 7182.94 & 182.45 & $7.96 \mathrm{E}+14$ & $28.51 \%$ & $1.15 \mathrm{E}+21$ \\
\hline & A-20 & -20.82 & 6125.34 & 155.58 & $6.53 \mathrm{E}+14$ & $23.87 \%$ & $9.46 \mathrm{E}+20$ \\
\hline & MAX & NA & 10382.49 & 263.72 & $1.22 \mathrm{E}+15$ & $45.00 \%$ & $1.91 \mathrm{E}+21$ \\
\hline & MIN & NA & 5020.40 & 127.52 & $5.27 \mathrm{E}+14$ & $19.36 \%$ & $7.58 \mathrm{E}+20$ \\
\hline & AVG & NA & 8801.27 & 223.55 & $1.02 \mathrm{E}+15$ & $36.36 \%$ & $1.51 \mathrm{E}+21$ \\
\hline
\end{tabular}

(a) Elevations for MCNP cells are reported at cell centroids relative to the modeled core axial midplane at 24.0 inches. 
Table 14. Cycle 143A, MCNP-Calculated HGR and Neutron Flux for AFIP-1 fuel, 0.0 EFPD (BOC), Center Lobe Power at 24.3 MW. ${ }^{3}$

\begin{tabular}{|c|c|c|c|c|c|}
\hline $\begin{array}{c}\text { Plate } \\
\text { ID }\end{array}$ & Cell & $\begin{array}{c}\text { MCNP Cell } \\
\text { Elevation }^{(\text {a) }} \\
\text { (inches) }^{2}\end{array}$ & $\begin{array}{c}\text { Fission } \\
\text { Power Density } \\
(\mathrm{W} / \mathrm{cc})\end{array}$ & $\begin{array}{c}\text { Surface } \\
\text { Heat Flux } \\
\left(\mathrm{W} / \mathrm{cm}^{2}\right)\end{array}$ & $\begin{array}{c}\text { Neutron } \\
\text { Flux } \\
\left(\mathrm{n} / \mathrm{cm}^{2} \mathrm{sec}\right)\end{array}$ \\
\hline & A-1 & 20.82 & 3890.90 & 98.83 & $4.10 \mathrm{E}+14$ \\
& A-2 & 18.76 & 4863.94 & 123.54 & $5.31 \mathrm{E}+14$ \\
& A-3 & 16.70 & 5534.06 & 140.57 & $6.41 \mathrm{E}+14$ \\
& A-4 & 14.64 & 6229.52 & 158.23 & $7.40 \mathrm{E}+14$ \\
& A-5 & 12.58 & 6891.87 & 175.05 & $8.38 \mathrm{E}+14$ \\
& A-6 & 10.52 & 7354.62 & 186.81 & $9.14 \mathrm{E}+14$ \\
& A-7 & 8.46 & 7759.92 & 197.10 & $9.79 \mathrm{E}+14$ \\
& A-8 & 6.40 & 8088.70 & 205.45 & $1.02 \mathrm{E}+15$ \\
& A-9 & 4.34 & 8376.20 & 212.76 & $1.05 \mathrm{E}+15$ \\
& A-10 & 2.28 & 8448.91 & 214.60 & $1.08 \mathrm{E}+15$ \\
\hline \hline & A-11 & -2.28 & 8461.54 & 214.92 & $1.08 \mathrm{E}+15$ \\
& A-12 & -4.34 & 8459.08 & 214.86 & $1.08 \mathrm{E}+15$ \\
& A-13 & -6.40 & 8228.54 & 209.00 & $1.05 \mathrm{E}+15$ \\
& A-14 & -8.46 & 7950.58 & 201.94 & $1.02 \mathrm{E}+15$ \\
1B5 & A-15 & -10.52 & 7627.84 & 193.75 & $9.63 \mathrm{E}+14$ \\
& A-16 & -12.58 & 7278.26 & 184.87 & $9.03 \mathrm{E}+14$ \\
& A-17 & -14.64 & 6733.17 & 171.02 & $8.24 \mathrm{E}+14$ \\
& A-18 & -16.70 & 6126.26 & 155.61 & $7.28 \mathrm{E}+14$ \\
& A-19 & -18.76 & 5444.06 & 138.28 & $6.21 \mathrm{E}+14$ \\
& A-20 & -20.82 & 4496.59 & 114.21 & $4.99 \mathrm{E}+14$ \\
\hline \hline & MAX & NA & 8461.54 & 214.92 & $1.08 \mathrm{E}+15$ \\
\hline & MIN & NA & 3890.90 & 98.83 & $4.10 \mathrm{E}+14$ \\
\hline & AVG & NA & 6912.23 & 175.57 & $8.48 \mathrm{E}+14$ \\
\hline
\end{tabular}

(a) Elevations for MCNP cells are reported at cell centroids relative to the modeled core axial midplane at 24.0 inches.

Table 15. Cycle 143A, MCNP-Calculated HGR, Neutron Flux, Depletion, and Fission Density for AFIP-1 fuel, 18.0 EFPD (MOC1), Center Lobe Power at 24.3 MW (70.0 EFPD Cumulative). ${ }^{3}$

\begin{tabular}{|c|c|c|c|c|c|c|c|}
\hline $\begin{array}{c}\text { Plate } \\
\text { ID }\end{array}$ & Cell & $\begin{array}{c}\text { MCNP Cell } \\
\text { Elevation } \\
\text { (inches) }\end{array}$ & $\begin{array}{c}\text { Fission } \\
\text { Power Density } \\
(\mathrm{W} / \mathrm{cc})\end{array}$ & $\begin{array}{c}\text { Surface } \\
\text { Heat Flux } \\
\left(\mathrm{W} / \mathrm{cm}^{2}\right)\end{array}$ & $\begin{array}{c}\text { Neutron } \\
\text { Flux } \\
\left(\mathrm{n} / \mathrm{cm}^{2} \mathrm{sec}\right)\end{array}$ & $\begin{array}{c}\% \text { Depletion } \\
\text { U-235 } \\
(\%)\end{array}$ & $\begin{array}{c}\text { Fission } \\
\text { Density } \\
\text { (fissions/cc) }\end{array}$ \\
\hline & A-1 & 20.82 & 4554.58 & 115.69 & $4.77 \mathrm{E}+14$ & $24.52 \%$ & $9.63 \mathrm{E}+20$ \\
& A-2 & 18.76 & 5502.55 & 139.76 & $6.11 \mathrm{E}+14$ & $30.44 \%$ & $1.21 \mathrm{E}+21$ \\
& A-3 & 16.70 & 6197.77 & 157.42 & $7.22 \mathrm{E}+14$ & $36.32 \%$ & $1.47 \mathrm{E}+21$ \\
& A-4 & 14.64 & 6842.80 & 173.81 & $8.23 \mathrm{E}+14$ & $40.89 \%$ & $1.68 \mathrm{E}+21$ \\
1T2 & A-5 & 12.58 & 7318.39 & 185.89 & $9.09 \mathrm{E}+14$ & $45.02 \%$ & $1.87 \mathrm{E}+21$ \\
& A-6 & 10.52 & 7803.11 & 198.20 & $9.70 \mathrm{E}+14$ & $47.78 \%$ & $2.00 \mathrm{E}+21$ \\
& A-7 & 8.46 & 7917.72 & 201.11 & $1.02 \mathrm{E}+15$ & $50.20 \%$ & $2.12 \mathrm{E}+21$ \\
& A-8 & 6.40 & 8129.28 & 206.48 & $1.05 \mathrm{E}+15$ & $51.79 \%$ & $2.20 \mathrm{E}+21$ \\
& A-9 & 4.34 & 8219.88 & 208.78 & $1.07 \mathrm{E}+15$ & $52.80 \%$ & $2.25 \mathrm{E}+21$ \\
& A-10 & 2.28 & 8232.13 & 209.10 & $1.08 \mathrm{E}+15$ & $54.35 \%$ & $2.33 \mathrm{E}+21$ \\
\hline \hline & A-11 & -2.28 & 8216.41 & 208.70 & $1.08 \mathrm{E}+15$ & $54.93 \%$ & $2.35 \mathrm{E}+21$ \\
& A-12 & -4.34 & 8194.31 & 208.14 & $1.08 \mathrm{E}+15$ & $53.52 \%$ & $2.28 \mathrm{E}+21$ \\
& A-13 & -6.40 & 8255.07 & 209.68 & $1.06 \mathrm{E}+15$ & $53.10 \%$ & $2.26 \mathrm{E}+21$ \\
& A-14 & -8.46 & 8061.70 & 204.77 & $1.04 \mathrm{E}+15$ & $52.00 \%$ & $2.21 \mathrm{E}+21$ \\
1B5 & A-15 & -10.52 & 7828.39 & 198.84 & $1.00 \mathrm{E}+15$ & $50.13 \%$ & $2.12 \mathrm{E}+21$ \\
& A-16 & -12.58 & 7481.32 & 190.03 & $9.52 \mathrm{E}+14$ & $47.82 \%$ & $2.00 \mathrm{E}+21$ \\
& A-17 & -14.64 & 7231.20 & 183.67 & $8.92 \mathrm{E}+14$ & $44.30 \%$ & $1.83 \mathrm{E}+21$ \\
& A-18 & -16.70 & 6630.70 & 168.42 & $8.03 \mathrm{E}+14$ & $40.42 \%$ & $1.66 \mathrm{E}+21$ \\
& A-19 & -18.76 & 5981.95 & 151.94 & $6.96 \mathrm{E}+14$ & $35.46 \%$ & $1.43 \mathrm{E}+21$ \\
& A-20 & -20.82 & 5140.77 & 130.58 & $5.63 \mathrm{E}+14$ & $29.74 \%$ & $1.18 \mathrm{E}+21$ \\
\hline \hline & MAX & NA & 8255.07 & 209.68 & $1.08 \mathrm{E}+15$ & $54.93 \%$ & $2.35 \mathrm{E}+21$ \\
\hline & MIN & NA & 4554.58 & 115.69 & $4.77 \mathrm{E}+14$ & $24.52 \%$ & $9.63 \mathrm{E}+20$ \\
\hline & AVG & NA & 7187.00 & 182.55 & $8.95 \mathrm{E}+14$ & $44.78 \%$ & $1.87 \mathrm{E}+21$ \\
\hline
\end{tabular}

(a) Elevations for MCNP cells are reported at cell centroids relative to the modeled core axial midplane at 24.0 inches. 
Table 16. Cycle 143A, MCNP-Calculated HGR, Neutron Flux, Depletion, and Fission Density for AFIP-1 fuel, 38.0 EFPD (MOC2), Center Lobe Power at 24.3 MW (90.0 EFPD Cumulative). ${ }^{3}$

\begin{tabular}{|c|c|c|c|c|c|c|c|}
\hline $\begin{array}{c}\text { Plate } \\
\text { ID }\end{array}$ & Cell & $\begin{array}{c}\text { MCNP Cell } \\
\text { Elevation } \\
\text { (inches) }\end{array}$ & $\begin{array}{c}\text { Fission } \\
\text { Power Density } \\
\text { (W/cc) }\end{array}$ & $\begin{array}{c}\text { Surface } \\
\text { Heat Flux } \\
\left(\mathrm{W} / \mathrm{cm}^{2}\right)\end{array}$ & $\begin{array}{c}\text { Neutron } \\
\text { Flux } \\
\left(\mathrm{n} / \mathrm{cm}^{2} \mathrm{sec}\right)\end{array}$ & $\begin{array}{c}\text { \% Depletion } \\
\text { U-235 } \\
(\%)\end{array}$ & $\begin{array}{c}\text { Fission } \\
\text { Density } \\
\text { (fissions/cc) }\end{array}$ \\
\hline & A-1 & 20.82 & 4654.02 & 118.21 & $5.14 \mathrm{E}+14$ & $31.16 \%$ & $1.23 \mathrm{E}+21$ \\
& A-2 & 18.76 & 5628.14 & 142.95 & $6.57 \mathrm{E}+14$ & $38.22 \%$ & $1.54 \mathrm{E}+21$ \\
& A-3 & 16.70 & 6250.59 & 158.77 & $7.67 \mathrm{E}+14$ & $44.86 \%$ & $1.84 \mathrm{E}+21$ \\
& A-4 & 14.64 & 6707.86 & 170.38 & $8.60 \mathrm{E}+14$ & $50.07 \%$ & $2.08 \mathrm{E}+21$ \\
& A-5 & 12.58 & 7001.06 & 177.83 & $9.41 \mathrm{E}+14$ & $54.62 \%$ & $2.30 \mathrm{E}+21$ \\
& A-6 & 10.52 & 7139.92 & 181.35 & $9.90 \mathrm{E}+14$ & $57.83 \%$ & $2.46 \mathrm{E}+21$ \\
& A-7 & 8.46 & 7236.66 & 183.81 & $1.03 \mathrm{E}+15$ & $60.25 \%$ & $2.59 \mathrm{E}+21$ \\
& A-8 & 6.40 & 7293.12 & 185.25 & $1.05 \mathrm{E}+15$ & $62.05 \%$ & $2.68 \mathrm{E}+21$ \\
& A-9 & 4.34 & 7304.27 & 185.53 & $1.07 \mathrm{E}+15$ & $63.10 \%$ & $2.73 \mathrm{E}+21$ \\
& A-10 & 2.28 & 7276.95 & 184.83 & $1.07 \mathrm{E}+15$ & $64.56 \%$ & $2.81 \mathrm{E}+21$ \\
\hline \hline & A-11 & -2.28 & 7187.92 & 182.57 & $1.07 \mathrm{E}+15$ & $65.12 \%$ & $2.84 \mathrm{E}+21$ \\
& A-12 & -4.34 & 7257.87 & 184.35 & $1.07 \mathrm{E}+15$ & $63.75 \%$ & $2.77 \mathrm{E}+21$ \\
& A-13 & -6.40 & 7287.43 & 185.10 & $1.06 \mathrm{E}+15$ & $63.37 \%$ & $2.75 \mathrm{E}+21$ \\
& A-14 & -8.46 & 7222.47 & 183.45 & $1.05 \mathrm{E}+15$ & $62.16 \%$ & $2.68 \mathrm{E}+21$ \\
1B5 & A-15 & -10.52 & 7148.67 & 181.58 & $1.02 \mathrm{E}+15$ & $60.07 \%$ & $2.58 \mathrm{E}+21$ \\
& A-16 & -12.58 & 7010.51 & 178.07 & $9.77 \mathrm{E}+14$ & $57.49 \%$ & $2.44 \mathrm{E}+21$ \\
& A-17 & -14.64 & 6870.65 & 174.51 & $9.16 \mathrm{E}+14$ & $53.86 \%$ & $2.26 \mathrm{E}+21$ \\
& A-18 & -16.70 & 6411.44 & 162.85 & $8.27 \mathrm{E}+14$ & $49.35 \%$ & $2.05 \mathrm{E}+21$ \\
& A-19 & -18.76 & 5911.26 & 150.15 & $7.21 \mathrm{E}+14$ & $43.74 \%$ & $1.79 \mathrm{E}+21$ \\
& A-20 & -20.82 & 5292.08 & 134.42 & $5.92 \mathrm{E}+14$ & $37.06 \%$ & $1.49 \mathrm{E}+21$ \\
\hline \hline & MAX & NA & 7304.27 & 185.53 & $1.07 \mathrm{E}+15$ & $65.12 \%$ & $2.84 \mathrm{E}+21$ \\
\hline & MIN & NA & 4654.02 & 118.21 & $5.14 \mathrm{E}+14$ & $31.16 \%$ & $1.23 \mathrm{E}+21$ \\
\hline & AVG & NA & 6704.64 & 170.30 & $9.12 \mathrm{E}+14$ & $54.13 \%$ & $2.30 \mathrm{E}+21$ \\
\hline
\end{tabular}

(a) Elevations for MCNP cells are reported at cell centroids relative to the modeled core axial midplane at 24.0 inches.

Table 17. Cycle 143A, MCNP-Calculated HGR, Neutron Flux, Depletion, and Fission Density for AFIP-1 fuel, 48.9 EFPD (EOC), Center Lobe Power at 24.3 MW (100.9 EFPD Cumulative). ${ }^{3}$

\begin{tabular}{|c|c|c|c|c|c|c|c|}
\hline $\begin{array}{c}\text { Plate } \\
\text { ID }\end{array}$ & Cell & $\begin{array}{c}\text { MCNP Cell } \\
\text { Elevation } \\
\text { (inches) }\end{array}$ & $\begin{array}{c}\text { Fission } \\
\text { Power Density } \\
(\mathrm{W} / \mathrm{cc})\end{array}$ & $\begin{array}{c}\text { Surface } \\
\text { Heat Flux } \\
\left(\mathrm{W} / \mathrm{cm}^{2}\right)\end{array}$ & $\begin{array}{c}\text { Neutron } \\
\text { Flux } \\
\left(\mathrm{n} / \mathrm{cm}^{2} \mathrm{sec}\right)\end{array}$ & $\begin{array}{c}\% \text { Depletion } \\
\text { U-235 } \\
(\%)\end{array}$ & $\begin{array}{c}\text { Fission } \\
\text { Density } \\
\text { (fissions/cc) }\end{array}$ \\
\hline & A-1 & 20.82 & 4495.47 & 114.18 & $5.12 \mathrm{E}+14$ & $34.88 \%$ & $1.39 \mathrm{E}+21$ \\
& A-2 & 18.76 & 5367.10 & 136.32 & $6.53 \mathrm{E}+14$ & $42.60 \%$ & $1.72 \mathrm{E}+21$ \\
& A-3 & 16.70 & 5897.26 & 149.79 & $7.65 \mathrm{E}+14$ & $49.62 \%$ & $2.04 \mathrm{E}+21$ \\
& A-4 & 14.64 & 6288.21 & 159.72 & $8.59 \mathrm{E}+14$ & $55.00 \%$ & $2.30 \mathrm{E}+21$ \\
1T2 & A-5 & 12.58 & 6473.07 & 164.42 & $9.38 \mathrm{E}+14$ & $59.67 \%$ & $2.53 \mathrm{E}+21$ \\
& A-6 & 10.52 & 6554.10 & 166.47 & $9.87 \mathrm{E}+14$ & $62.90 \%$ & $2.70 \mathrm{E}+21$ \\
& A-7 & 8.46 & 6597.78 & 167.58 & $1.02 \mathrm{E}+15$ & $65.32 \%$ & $2.82 \mathrm{E}+21$ \\
& A-8 & 6.40 & 6608.62 & 167.86 & $1.04 \mathrm{E}+15$ & $67.14 \%$ & $2.91 \mathrm{E}+21$ \\
& A-9 & 4.34 & 6607.82 & 167.84 & $1.06 \mathrm{E}+15$ & $68.12 \%$ & $2.97 \mathrm{E}+21$ \\
& A-10 & 2.28 & 6575.32 & 167.01 & $1.06 \mathrm{E}+15$ & $69.56 \%$ & $3.05 \mathrm{E}+21$ \\
\hline \hline & A-11 & -2.28 & 6492.39 & 164.91 & $1.07 \mathrm{E}+15$ & $70.03 \%$ & $3.07 \mathrm{E}+21$ \\
& A-12 & -4.34 & 6584.56 & 167.25 & $1.06 \mathrm{E}+15$ & $68.71 \%$ & $3.00 \mathrm{E}+21$ \\
& A-13 & -6.40 & 6618.58 & 168.11 & $1.06 \mathrm{E}+15$ & $68.37 \%$ & $2.99 \mathrm{E}+21$ \\
& A-14 & -8.46 & 6586.40 & 167.29 & $1.04 \mathrm{E}+15$ & $67.16 \%$ & $2.92 \mathrm{E}+21$ \\
1B5 & A-15 & -10.52 & 6553.45 & 166.46 & $1.01 \mathrm{E}+15$ & $65.07 \%$ & $2.81 \mathrm{E}+21$ \\
& A-16 & -12.58 & 6462.22 & 164.14 & $9.74 \mathrm{E}+14$ & $62.49 \%$ & $2.67 \mathrm{E}+21$ \\
& A-17 & -14.64 & 6368.38 & 161.76 & $9.14 \mathrm{E}+14$ & $58.88 \%$ & $2.49 \mathrm{E}+21$ \\
& A-18 & -16.70 & 6015.40 & 152.79 & $8.25 \mathrm{E}+14$ & $54.10 \%$ & $2.26 \mathrm{E}+21$ \\
& A-19 & -18.76 & 5606.24 & 142.40 & $7.19 \mathrm{E}+14$ & $48.25 \%$ & $1.98 \mathrm{E}+21$ \\
& A-20 & -20.82 & 5062.08 & 128.58 & $5.90 \mathrm{E}+14$ & $41.21 \%$ & $1.66 \mathrm{E}+21$ \\
\hline \hline & MAX & NA & 6618.58 & 168.11 & $1.07 \mathrm{E}+15$ & $70.03 \%$ & $3.07 \mathrm{E}+21$ \\
\hline & MIN & NA & 4495.47 & 114.18 & $5.12 \mathrm{E}+14$ & $34.88 \%$ & $1.39 \mathrm{E}+21$ \\
\hline & AVG & NA & 6190.72 & 157.24 & $9.09 \mathrm{E}+14$ & $58.95 \%$ & $2.51 \mathrm{E}+21$ \\
\hline
\end{tabular}

(a) Elevations for MCNP cells are reported at cell centroids relative to the modeled core axial midplane at 24.0 inches. 
Table 18. Cycle 143B, MCNP-Calculated HGR and Neutron Flux for AFIP-1 fuel, 0.0 EFPD (BOC), Center Lobe Power at 24.9 MW. ${ }^{3}$

\begin{tabular}{|c|c|c|c|c|c|}
\hline $\begin{array}{c}\text { Plate } \\
\text { ID }\end{array}$ & Cell & $\begin{array}{c}\text { MCNP Cell } \\
\text { Elevation }^{(\text {a) }} \\
\text { (inches) }^{2}\end{array}$ & $\begin{array}{c}\text { Fission } \\
\text { Power Density } \\
(\mathrm{W} / \mathrm{cc})\end{array}$ & $\begin{array}{c}\text { Surface } \\
\text { Heat Flux } \\
\left(\mathrm{W} / \mathrm{cm}^{2}\right)\end{array}$ & $\begin{array}{c}\text { Neutron } \\
\text { Flux } \\
\left(\mathrm{n} / \mathrm{cm}^{2} \mathrm{sec}\right)\end{array}$ \\
\hline & A-1 & 20.82 & 3152.49 & 80.07 & $4.28 \mathrm{E}+14$ \\
& A-2 & 18.76 & 3635.94 & 92.35 & $5.53 \mathrm{E}+14$ \\
& A-3 & 16.70 & 4097.70 & 104.08 & $6.71 \mathrm{E}+14$ \\
& A-4 & 14.64 & 4483.23 & 113.87 & $7.79 \mathrm{E}+14$ \\
& A-5 & 12.58 & 4654.66 & 118.23 & $8.64 \mathrm{E}+14$ \\
& A-6 & 10.52 & 5006.52 & 127.17 & $9.50 \mathrm{E}+14$ \\
& A-7 & 8.46 & 4976.79 & 126.41 & $1.01 \mathrm{E}+15$ \\
& A-8 & 6.40 & 5112.12 & 129.85 & $1.05 \mathrm{E}+15$ \\
& A-9 & 4.34 & 5186.15 & 131.73 & $1.09 \mathrm{E}+15$ \\
& A-10 & 2.28 & 5288.18 & 134.32 & $1.10 \mathrm{E}+15$ \\
\hline \hline & A-11 & -2.28 & 5245.47 & 133.23 & $1.10 \mathrm{E}+15$ \\
& A-12 & -4.34 & 5214.73 & 132.45 & $1.10 \mathrm{E}+15$ \\
& A-13 & -6.40 & 5093.46 & 129.37 & $1.07 \mathrm{E}+15$ \\
& A-14 & -8.46 & 5066.70 & 128.69 & $1.04 \mathrm{E}+15$ \\
1B5 & A-15 & -10.52 & 4965.31 & 126.12 & $9.98 \mathrm{E}+14$ \\
& A-16 & -12.58 & 4916.91 & 124.89 & $9.36 \mathrm{E}+14$ \\
& A-17 & -14.64 & 4617.87 & 117.29 & $8.59 \mathrm{E}+14$ \\
& A-18 & -16.70 & 4403.45 & 111.85 & $7.67 \mathrm{E}+14$ \\
& A-19 & -18.76 & 4102.00 & 104.19 & $6.54 \mathrm{E}+14$ \\
& A-20 & -20.82 & 3608.31 & 91.65 & $5.22 \mathrm{E}+14$ \\
\hline \hline & MAX & NA & 5288.18 & 134.32 & $1.10 \mathrm{E}+15$ \\
\hline & MIN & NA & 3152.49 & 80.07 & $4.28 \mathrm{E}+14$ \\
\hline & AVG & NA & 4641.40 & 117.89 & $8.77 \mathrm{E}+14$ \\
\hline
\end{tabular}

(a) Elevations for MCNP cells are reported at cell centroids relative to the modeled core axial midplane at 24.0 inches.

Table 19. Cycle 143B, MCNP-Calculated HGR, Neutron Flux, Depletion, and Fission Density for AFIP-1 fuel, 18.0 EFPD (MOC1), Center Lobe Power at 24.9 MW (118.9 EFPD Cumulative). ${ }^{3}$

\begin{tabular}{|c|c|c|c|c|c|c|c|}
\hline $\begin{array}{c}\text { Plate } \\
\text { ID }\end{array}$ & Cell & $\begin{array}{c}\text { MCNP Cell } \\
\text { Elevation } \\
\text { (inches) }\end{array}$ & $\begin{array}{c}\text { Fission } \\
\text { Power Density } \\
(\mathrm{W} / \mathrm{cc})\end{array}$ & $\begin{array}{c}\text { Surface } \\
\text { Heat Flux } \\
\left(\mathrm{W} / \mathrm{cm}^{2}\right)\end{array}$ & $\begin{array}{c}\text { Neutron } \\
\text { Flux } \\
\left(\mathrm{n} / \mathrm{cm}^{2} \mathrm{sec}\right)\end{array}$ & $\begin{array}{c}\% \text { Depletion } \\
\text { U-235 } \\
(\%)\end{array}$ & $\begin{array}{c}\text { Fission } \\
\text { Density } \\
\text { (fissions/cc) }\end{array}$ \\
\hline & A-1 & 20.82 & 3511.48 & 89.19 & $4.84 \mathrm{E}+14$ & $38.85 \%$ & $1.55 \mathrm{E}+21$ \\
& A-2 & 18.76 & 4018.57 & 102.07 & $6.16 \mathrm{E}+14$ & $47.04 \%$ & $1.91 \mathrm{E}+21$ \\
& A-3 & 16.70 & 4401.84 & 111.81 & $7.30 \mathrm{E}+14$ & $54.44 \%$ & $2.25 \mathrm{E}+21$ \\
& A-4 & 14.64 & 4686.84 & 119.05 & $8.34 \mathrm{E}+14$ & $60.12 \%$ & $2.53 \mathrm{E}+21$ \\
1T2 & A-5 & 12.58 & 4709.78 & 119.63 & $9.05 \mathrm{E}+14$ & $64.87 \%$ & $2.77 \mathrm{E}+21$ \\
& A-6 & 10.52 & 4894.49 & 124.32 & $9.73 \mathrm{E}+14$ & $68.33 \%$ & $2.95 \mathrm{E}+21$ \\
& A-7 & 8.46 & 4913.92 & 124.81 & $1.02 \mathrm{E}+15$ & $70.64 \%$ & $3.08 \mathrm{E}+21$ \\
& A-8 & 6.40 & 4950.67 & 125.75 & $1.04 \mathrm{E}+15$ & $72.52 \%$ & $3.18 \mathrm{E}+21$ \\
& A-9 & 4.34 & 4980.95 & 126.52 & $1.07 \mathrm{E}+15$ & $73.55 \%$ & $3.24 \mathrm{E}+21$ \\
& A-10 & 2.28 & 4932.20 & 125.28 & $1.06 \mathrm{E}+15$ & $74.99 \%$ & $3.32 \mathrm{E}+21$ \\
\hline \hline & A-11 & -2.28 & 4832.84 & 122.75 & $1.07 \mathrm{E}+15$ & $75.39 \%$ & $3.34 \mathrm{E}+21$ \\
& A-12 & -4.34 & 4895.47 & 124.34 & $1.07 \mathrm{E}+15$ & $74.11 \%$ & $3.27 \mathrm{E}+21$ \\
& A-13 & -6.40 & 4881.41 & 123.99 & $1.06 \mathrm{E}+15$ & $73.67 \%$ & $3.25 \mathrm{E}+21$ \\
& A-14 & -8.46 & 4889.80 & 124.20 & $1.04 \mathrm{E}+15$ & $72.48 \%$ & $3.18 \mathrm{E}+21$ \\
1B5 & A-15 & -10.52 & 4860.54 & 123.46 & $1.00 \mathrm{E}+15$ & $70.37 \%$ & $3.07 \mathrm{E}+21$ \\
& A-16 & -12.58 & 4734.13 & 120.25 & $9.55 \mathrm{E}+14$ & $67.83 \%$ & $2.93 \mathrm{E}+21$ \\
& A-17 & -14.64 & 4735.99 & 120.29 & $8.90 \mathrm{E}+14$ & $64.09 \%$ & $2.72 \mathrm{E}+21$ \\
& A-18 & -16.70 & 4602.38 & 116.90 & $8.10 \mathrm{E}+14$ & $59.17 \%$ & $2.49 \mathrm{E}+21$ \\
& A-19 & -18.76 & 4340.38 & 110.25 & $7.04 \mathrm{E}+14$ & $53.12 \%$ & $2.19 \mathrm{E}+21$ \\
& A-20 & -20.82 & 3881.42 & 98.59 & $5.74 \mathrm{E}+14$ & $45.63 \%$ & $1.85 \mathrm{E}+21$ \\
\hline \hline & MAX & NA & 4980.95 & 126.52 & $1.07 \mathrm{E}+15$ & $75.39 \%$ & $3.34 \mathrm{E}+21$ \\
\hline & MIN & NA & 3511.48 & 89.19 & $4.84 \mathrm{E}+14$ & $38.85 \%$ & $1.55 \mathrm{E}+21$ \\
\hline & AVG & NA & 4632.76 & 117.67 & $8.96 \mathrm{E}+14$ & $64.06 \%$ & $2.75 \mathrm{E}+21$ \\
\hline
\end{tabular}

(a) Elevations for MCNP cells are reported at cell centroids relative to the modeled core axial midplane at 24.0 inches. 
Table 20. Cycle 143B, MCNP-Calculated HGR, Neutron Flux, Depletion, and Fission Density for AFIP-1 fuel, 38.0 EFPD (MOC2), Center Lobe Power at 24.9 MW (138.9 EFPD Cumulative). ${ }^{3}$

\begin{tabular}{|c|c|c|c|c|c|c|c|}
\hline $\begin{array}{c}\text { Plate } \\
\text { ID }\end{array}$ & Cell & $\begin{array}{c}\text { MCNP Cell } \\
\text { Elevation }^{(a)} \\
\text { (inches) }^{2}\end{array}$ & $\begin{array}{c}\text { Fission } \\
\text { Power Density } \\
(\mathrm{W} / \mathrm{cc})\end{array}$ & $\begin{array}{l}\text { Surface } \\
\text { Heat Flux } \\
\left(\mathrm{W} / \mathrm{cm}^{2}\right)\end{array}$ & $\begin{array}{c}\text { Neutron } \\
\text { Flux } \\
\left(\mathrm{n} / \mathrm{cm}^{2} \mathrm{sec}\right) \\
\end{array}$ & $\begin{array}{c}\text { \% Depletion } \\
\text { U-235 } \\
(\%)\end{array}$ & $\begin{array}{c}\text { Fission } \\
\text { Density } \\
\text { (fissions/cc) }\end{array}$ \\
\hline \multirow{10}{*}{$1 \mathrm{~T} 2$} & A-1 & 20.82 & 3642.74 & 92.53 & $5.09 \mathrm{E}+14$ & $43.72 \%$ & $1.75 \mathrm{E}+21$ \\
\hline & A-2 & 18.76 & 4187.51 & 106.36 & $6.48 \mathrm{E}+14$ & $52.40 \%$ & $2.14 \mathrm{E}+21$ \\
\hline & A-3 & 16.70 & 4404.04 & 111.86 & $7.67 \mathrm{E}+14$ & $60.07 \%$ & $2.51 \mathrm{E}+21$ \\
\hline & A-4 & 14.64 & 4638.06 & 117.81 & $8.62 \mathrm{E}+14$ & $65.88 \%$ & $2.81 \mathrm{E}+21$ \\
\hline & A-5 & 12.58 & 4582.66 & 116.40 & $9.31 \mathrm{E}+14$ & $70.50 \%$ & $3.05 \mathrm{E}+21$ \\
\hline & A-6 & 10.52 & 4673.34 & 118.70 & $9.89 \mathrm{E}+14$ & $73.98 \%$ & $3.24 \mathrm{E}+21$ \\
\hline & A-7 & 8.46 & 4634.67 & 117.72 & $1.03 \mathrm{E}+15$ & $76.20 \%$ & $3.37 \mathrm{E}+21$ \\
\hline & A-8 & 6.40 & 4561.74 & 115.87 & $1.05 \mathrm{E}+15$ & $78.01 \%$ & $3.47 \mathrm{E}+21$ \\
\hline & A-9 & 4.34 & 4493.33 & 114.13 & $1.06 \mathrm{E}+15$ & $79.01 \%$ & $3.53 \mathrm{E}+21$ \\
\hline & A-10 & 2.28 & 4517.96 & 114.76 & $1.06 \mathrm{E}+15$ & $80.31 \%$ & $3.61 \mathrm{E}+21$ \\
\hline \multirow{13}{*}{ 1B5 } & A-11 & -2.28 & 44418.80 & 112.24 & $1.06 \mathrm{E}+15$ & $80.59 \%$ & $3.62 \mathrm{E}+21$ \\
\hline & A-12 & -4.34 & 4461.50 & 113.32 & $1.06 \mathrm{E}+15$ & $79.44 \%$ & $3.56 \mathrm{E}+21$ \\
\hline & A-13 & -6.40 & 4538.84 & 115.29 & $1.06 \mathrm{E}+15$ & $79.00 \%$ & $3.54 \mathrm{E}+21$ \\
\hline & A-14 & -8.46 & 4487.38 & 113.98 & $1.04 \mathrm{E}+15$ & $77.89 \%$ & $3.47 \mathrm{E}+21$ \\
\hline & A-15 & -10.52 & 4528.31 & 115.02 & $1.01 \mathrm{E}+15$ & $75.84 \%$ & $3.35 \mathrm{E}+21$ \\
\hline & A-16 & -12.58 & 4618.70 & 117.31 & $9.72 \mathrm{E}+14$ & $73.33 \%$ & $3.20 \mathrm{E}+21$ \\
\hline & A-17 & -14.64 & 4514.57 & 114.67 & $9.10 \mathrm{E}+14$ & $69.78 \%$ & $3.00 \mathrm{E}+21$ \\
\hline & A-18 & -16.70 & 4517.01 & 114.73 & $8.35 \mathrm{E}+14$ & $64.89 \%$ & $2.75 \mathrm{E}+21$ \\
\hline & A-19 & -18.76 & 4310.05 & 109.48 & $7.27 \mathrm{E}+14$ & $58.73 \%$ & $2.45 \mathrm{E}+21$ \\
\hline & A-20 & -20.82 & 3988.12 & 101.30 & $5.96 \mathrm{E}+14$ & $50.85 \%$ & $2.07 \mathrm{E}+21$ \\
\hline & MAX & NA & 4673.34 & 118.70 & $1.06 \mathrm{E}+15$ & $80.59 \%$ & $3.62 \mathrm{E}+21$ \\
\hline & MIN & NA & 3642.74 & 92.53 & $5.09 \mathrm{E}+14$ & $43.72 \%$ & $1.75 \mathrm{E}+21$ \\
\hline & AVG & NA & 4435.97 & 112.67 & $9.09 \mathrm{E}+14$ & $69.52 \%$ & $3.02 \mathrm{E}+21$ \\
\hline
\end{tabular}

(a) Elevations for MCNP cells are reported at cell centroids relative to the modeled core axial midplane at 24.0 inches.

Table 21. Cycle 143B, MCNP-Calculated HGR, Neutron Flux, Depletion, and Fission Density for AFIP-1 fuel, 57.3 EFPD (EOC), Center Lobe Power at 24.9 MW (158.2 EFPD Cumulative). ${ }^{3}$

\begin{tabular}{|c|c|c|c|c|c|c|c|}
\hline $\begin{array}{c}\text { Plate } \\
\text { ID }\end{array}$ & Cell & $\begin{array}{c}\text { MCNP Cell } \\
\text { Elevation } \\
\text { (inches) }\end{array}$ & $\begin{array}{c}\text { Fission } \\
\text { Power Density } \\
(\text { W/cc) }\end{array}$ & $\begin{array}{c}\text { Surface } \\
\text { Heat Flux } \\
\left(\mathrm{W} / \mathrm{cm}^{2}\right)\end{array}$ & $\begin{array}{c}\text { Neutron } \\
\text { Flux } \\
\left(\mathrm{n} / \mathrm{cm}^{2} \mathrm{sec}\right)\end{array}$ & $\begin{array}{c}\% \text { Depletion } \\
\text { U-235 } \\
(\%)\end{array}$ & $\begin{array}{c}\text { Fission } \\
\text { Density } \\
\text { (fissions/cc) }\end{array}$ \\
\hline & A-1 & 20.82 & 3746.04 & 95.15 & $5.07 \mathrm{E}+14$ & $48.61 \%$ & $1.96 \mathrm{E}+21$ \\
& A-2 & 18.76 & 4327.45 & 109.92 & $6.46 \mathrm{E}+14$ & $57.76 \%$ & $2.38 \mathrm{E}+21$ \\
& A-3 & 16.70 & 4579.03 & 116.31 & $7.64 \mathrm{E}+14$ & $65.46 \%$ & $2.76 \mathrm{E}+21$ \\
& A-4 & 14.64 & 4869.80 & 123.69 & $8.59 \mathrm{E}+14$ & $71.27 \%$ & $3.07 \mathrm{E}+21$ \\
& A-5 & 12.58 & 4833.14 & 122.76 & $9.26 \mathrm{E}+14$ & $75.64 \%$ & $3.31 \mathrm{E}+21$ \\
& A-6 & 10.52 & 4961.85 & 126.03 & $9.86 \mathrm{E}+14$ & $79.00 \%$ & $3.51 \mathrm{E}+21$ \\
& A-7 & 8.46 & 4916.25 & 124.87 & $1.02 \mathrm{E}+15$ & $81.06 \%$ & $3.63 \mathrm{E}+21$ \\
& A-8 & 6.40 & 4877.30 & 123.88 & $1.04 \mathrm{E}+15$ & $82.67 \%$ & $3.73 \mathrm{E}+21$ \\
& A-9 & 4.34 & 4805.66 & 122.06 & $1.06 \mathrm{E}+15$ & $83.52 \%$ & $3.79 \mathrm{E}+21$ \\
& A-10 & 2.28 & 4806.01 & 122.07 & $1.06 \mathrm{E}+15$ & $84.75 \%$ & $3.87 \mathrm{E}+21$ \\
\hline \hline & A-11 & -2.28 & 4724.65 & 120.01 & $1.06 \mathrm{E}+15$ & $84.91 \%$ & $3.88 \mathrm{E}+21$ \\
& A-12 & -4.34 & 4783.87 & 121.51 & $1.06 \mathrm{E}+15$ & $83.90 \%$ & $3.81 \mathrm{E}+21$ \\
& A-13 & -6.40 & 4882.71 & 124.02 & $1.06 \mathrm{E}+15$ & $83.56 \%$ & $3.80 \mathrm{E}+21$ \\
& A-14 & -8.46 & 4802.45 & 121.98 & $1.03 \mathrm{E}+15$ & $82.48 \%$ & $3.72 \mathrm{E}+21$ \\
1B5 & A-15 & -10.52 & 4830.98 & 122.71 & $1.00 \mathrm{E}+15$ & $80.59 \%$ & $3.61 \mathrm{E}+21$ \\
& A-16 & -12.58 & 4910.92 & 124.74 & $9.69 \mathrm{E}+14$ & $78.32 \%$ & $3.47 \mathrm{E}+21$ \\
& A-17 & -14.64 & 4775.40 & 121.30 & $9.06 \mathrm{E}+14$ & $74.88 \%$ & $3.26 \mathrm{E}+21$ \\
& A-18 & -16.70 & 4743.98 & 120.50 & $8.33 \mathrm{E}+14$ & $70.17 \%$ & $3.01 \mathrm{E}+21$ \\
& A-19 & -18.76 & 4480.04 & 113.79 & $7.23 \mathrm{E}+14$ & $64.02 \%$ & $2.69 \mathrm{E}+21$ \\
& A-20 & -20.82 & 4121.47 & 104.69 & $5.94 \mathrm{E}+14$ & $56.01 \%$ & $2.30 \mathrm{E}+21$ \\
\hline \hline & MAX & NA & 4961.85 & 126.03 & $1.06 \mathrm{E}+15$ & $84.91 \%$ & $3.88 \mathrm{E}+21$ \\
\hline & MIN & NA & 3746.04 & 95.15 & $5.07 \mathrm{E}+14$ & $48.61 \%$ & $1.96 \mathrm{E}+21$ \\
\hline & AVG & NA & 4688.95 & 119.10 & $9.05 \mathrm{E}+14$ & $74.43 \%$ & $3.28 \mathrm{E}+21$ \\
\hline
\end{tabular}

(a) Elevations for MCNP cells are reported at cell centroids relative to the modeled core axial midplane at 24.0 inches. 
Table 22. Corrected fission power density (W/cc) and fission density (fissions/cc) for ATR Cycle 142B.

\begin{tabular}{|c|c|c|c|c|c|c|c|c|}
\hline \multirow[b]{2}{*}{$\begin{array}{c}\text { Plate } \\
\text { ID }\end{array}$} & \multirow[b]{2}{*}{ Cell } & \multirow{2}{*}{$\begin{array}{c}\text { BOC 142B } \\
\\
\text { Fission } \\
\text { Power Density } \\
(\mathrm{W} / \mathrm{cc})\end{array}$} & \multicolumn{2}{|c|}{ MOC1 142B } & \multicolumn{2}{|c|}{ MOC2 142B } & \multicolumn{2}{|c|}{ EOC 142B } \\
\hline & & & $\begin{array}{l}\text { Fission } \\
\text { Power } \\
\text { Density } \\
(\mathrm{W} / \mathrm{cc})\end{array}$ & $\begin{array}{c}\text { Fission } \\
\text { Density } \\
\text { (fissions/cc) }\end{array}$ & $\begin{array}{l}\text { Fission } \\
\text { Power } \\
\text { Density } \\
(\mathrm{W} / \mathrm{cc})\end{array}$ & $\begin{array}{c}\text { Fission } \\
\text { Density } \\
\text { (fissions/cc) }\end{array}$ & $\begin{array}{l}\text { Fission } \\
\text { Power } \\
\text { Density } \\
(\mathrm{W} / \mathrm{cc})\end{array}$ & $\begin{array}{c}\text { Fission } \\
\text { Density } \\
\text { (fissions/cc) }\end{array}$ \\
\hline \multirow{10}{*}{$1 \mathrm{~T} 2$} & A-1 & 4116.02 & 4125.37 & $1.94 \mathrm{E}+20$ & 4817.30 & $3.61 \mathrm{E}+20$ & 4543.35 & $6.86 \mathrm{E}+20$ \\
\hline & A-2 & 5220.03 & 5235.59 & $2.46 \mathrm{E}+20$ & 6025.53 & $4.58 \mathrm{E}+20$ & 5619.51 & $8.64 \mathrm{E}+20$ \\
\hline & A-3 & 6536.71 & 6463.76 & $3.08 \mathrm{E}+20$ & 7375.64 & $5.70 \mathrm{E}+20$ & 6683.64 & $1.07 \mathrm{E}+21$ \\
\hline & A-4 & 7517.32 & 7436.13 & $3.55 \mathrm{E}+20$ & 8407.60 & $6.56 \mathrm{E}+20$ & 7505.46 & $1.22 \mathrm{E}+21$ \\
\hline & A-5 & 8562.50 & 8232.31 & $4.04 \mathrm{E}+20$ & 9304.18 & $7.37 \mathrm{E}+20$ & 8175.38 & $1.36 \mathrm{E}+21$ \\
\hline & A-6 & 9321.95 & 8829.07 & $4.40 \mathrm{E}+20$ & 9846.67 & $7.97 \mathrm{E}+20$ & 8557.51 & $1.46 \mathrm{E}+21$ \\
\hline & A-7 & 9983.55 & 9407.51 & $4.71 \mathrm{E}+20$ & 10334.16 & $8.52 \mathrm{E}+20$ & 8891.83 & $1.55 \mathrm{E}+21$ \\
\hline & A-8 & 10468.92 & 9778.45 & $4.94 \mathrm{E}+20$ & 10581.38 & $8.90 \mathrm{E}+20$ & 9020.48 & $1.60 \mathrm{E}+21$ \\
\hline & A-9 & 10709.32 & 9870.26 & $5.05 \mathrm{E}+20$ & 10846.08 & $9.05 \mathrm{E}+20$ & 9210.85 & $1.64 \mathrm{E}+21$ \\
\hline & A-10 & 11249.07 & 10318.04 & $5.31 \mathrm{E}+20$ & 11194.54 & $9.49 \mathrm{E}+20$ & 9380.71 & $1.70 \mathrm{E}+21$ \\
\hline \multirow{10}{*}{ 1B5 } & A-11 & 11317.95 & 10066.39 & $5.34 \mathrm{E}+20$ & 10978.33 & $9.42 \mathrm{E}+20$ & 9147.57 & $1.68 \mathrm{E}+21$ \\
\hline & A-12 & 10728.51 & 9759.29 & $5.06 \mathrm{E}+20$ & 10654.89 & $9.01 \mathrm{E}+20$ & 9016.76 & $1.62 \mathrm{E}+21$ \\
\hline & A-13 & 10603.00 & 9736.03 & $5.00 \mathrm{E}+20$ & 10675.82 & $8.95 \mathrm{E}+20$ & 9019.86 & $1.61 \mathrm{E}+21$ \\
\hline & A-14 & 10395.43 & 9411.97 & $4.91 \mathrm{E}+20$ & 10455.75 & $8.72 \mathrm{E}+20$ & 8891.05 & $1.58 \mathrm{E}+21$ \\
\hline & A-15 & 9935.41 & 9109.07 & $4.69 \mathrm{E}+20$ & 9985.61 & $8.38 \mathrm{E}+20$ & 8562.01 & $1.51 \mathrm{E}+21$ \\
\hline & A-16 & 9234.17 & 8641.45 & $4.36 \mathrm{E}+20$ & 9502.76 & $7.86 \mathrm{E}+20$ & 8232.80 & $1.43 \mathrm{E}+21$ \\
\hline & A-17 & 8330.00 & 7813.37 & $3.93 \mathrm{E}+20$ & 8819.85 & $7.09 \mathrm{E}+20$ & 7783.00 & $1.30 \mathrm{E}+21$ \\
\hline & A-18 & 7355.31 & 7037.76 & $3.47 \mathrm{E}+20$ & 8055.62 & $6.32 \mathrm{E}+20$ & 7172.06 & $1.17 \mathrm{E}+21$ \\
\hline & A-19 & 6288.21 & 6057.16 & $2.97 \mathrm{E}+20$ & 6935.31 & $5.42 \mathrm{E}+20$ & 6328.58 & $1.01 \mathrm{E}+21$ \\
\hline & A-20 & 5066.25 & 5042.61 & $2.39 \mathrm{E}+20$ & 5787.39 & $4.43 \mathrm{E}+20$ & 5396.78 & $8.33 \mathrm{E}+20$ \\
\hline
\end{tabular}


Table 23. Corrected fission power density (W/cc) and fission density (fissions/cc) for ATR Cycle 143A.

\begin{tabular}{|c|c|c|c|c|c|c|c|c|}
\hline \multirow[b]{2}{*}{$\begin{array}{c}\text { Plate } \\
\text { ID }\end{array}$} & \multirow[b]{2}{*}{ Cell } & \multirow{2}{*}{$\begin{array}{c}\text { BOC 143A } \\
\\
\text { Fission } \\
\text { Power Density } \\
(\mathrm{W} / \mathrm{cc})\end{array}$} & \multicolumn{2}{|c|}{ MOC1 143A } & \multicolumn{2}{|c|}{ MOC2 143A } & \multicolumn{2}{|c|}{ EOC 143A } \\
\hline & & & $\begin{array}{l}\text { Fission } \\
\text { Power } \\
\text { Density } \\
(\mathrm{W} / \mathrm{cc})\end{array}$ & $\begin{array}{c}\text { Fission } \\
\text { Density } \\
\text { (fissions/cc) }\end{array}$ & $\begin{array}{l}\text { Fission } \\
\text { Power } \\
\text { Density } \\
(\mathrm{W} / \mathrm{cc})\end{array}$ & $\begin{array}{c}\text { Fission } \\
\text { Density } \\
\text { (fissions/cc) }\end{array}$ & $\begin{array}{l}\text { Fission } \\
\text { Power } \\
\text { Density } \\
(\mathrm{W} / \mathrm{cc})\end{array}$ & $\begin{array}{c}\text { Fission } \\
\text { Density } \\
\text { (fissions/cc) }\end{array}$ \\
\hline \multirow{10}{*}{$1 \mathrm{~T} 2$} & A-1 & 3521.18 & 4121.79 & $8.71 \mathrm{E}+20$ & 4211.79 & $1.12 \mathrm{E}+21$ & 4068.30 & $1.25 \mathrm{E}+21$ \\
\hline & A-2 & 4401.75 & 4979.68 & $1.10 \mathrm{E}+21$ & 5093.34 & $1.39 \mathrm{E}+21$ & 4857.10 & $1.56 \mathrm{E}+21$ \\
\hline & A-3 & 5008.20 & 5608.84 & $1.33 \mathrm{E}+21$ & 5656.64 & $1.66 \mathrm{E}+21$ & 5336.89 & $1.85 \mathrm{E}+21$ \\
\hline & A-4 & 5637.57 & 6192.58 & $1.52 \mathrm{E}+21$ & 6070.46 & $1.89 \mathrm{E}+21$ & 5690.69 & $2.08 \mathrm{E}+21$ \\
\hline & A-5 & 6236.98 & 6622.98 & $1.69 \mathrm{E}+21$ & 6335.80 & $2.08 \mathrm{E}+21$ & 5857.98 & $2.29 \mathrm{E}+21$ \\
\hline & A-6 & 6655.77 & 7061.64 & $1.81 \mathrm{E}+21$ & 6461.46 & $2.23 \mathrm{E}+21$ & 5931.31 & $2.44 \mathrm{E}+21$ \\
\hline & A-7 & 7022.55 & 7165.36 & $1.92 \mathrm{E}+21$ & 6549.02 & $2.34 \mathrm{E}+21$ & 5970.84 & $2.55 \mathrm{E}+21$ \\
\hline & A-8 & 7320.09 & 7356.82 & $1.99 \mathrm{E}+21$ & 6600.11 & $2.42 \mathrm{E}+21$ & 5980.65 & $2.64 \mathrm{E}+21$ \\
\hline & A-9 & 7580.27 & 7438.80 & $2.03 \mathrm{E}+21$ & 6610.20 & $2.47 \mathrm{E}+21$ & 5979.93 & $2.69 \mathrm{E}+21$ \\
\hline & A-10 & 7646.07 & 7449.89 & $2.10 \mathrm{E}+21$ & 6585.47 & $2.55 \mathrm{E}+21$ & 5950.52 & $2.76 \mathrm{E}+21$ \\
\hline \multirow{10}{*}{ 1B5 } & A-11 & 7455.11 & 7239.13 & $2.07 \mathrm{E}+21$ & 6332.97 & $2.50 \mathrm{E}+21$ & 5720.17 & $2.71 \mathrm{E}+21$ \\
\hline & A-12 & 7452.94 & 7219.66 & $2.01 \mathrm{E}+21$ & 6394.60 & $2.44 \mathrm{E}+21$ & 5801.37 & $2.65 \mathrm{E}+21$ \\
\hline & A-13 & 7249.81 & 7273.19 & $1.99 \mathrm{E}+21$ & 6420.64 & $2.43 \mathrm{E}+21$ & 5831.35 & $2.63 \mathrm{E}+21$ \\
\hline & A-14 & 7004.91 & 7102.82 & $1.94 \mathrm{E}+21$ & 6363.41 & $2.37 \mathrm{E}+21$ & 5803.00 & $2.57 \mathrm{E}+21$ \\
\hline & A-15 & 6720.57 & 6897.26 & $1.86 \mathrm{E}+21$ & 6298.39 & $2.27 \mathrm{E}+21$ & 5773.97 & $2.48 \mathrm{E}+21$ \\
\hline & A-16 & 6412.56 & 6591.47 & $1.76 \mathrm{E}+21$ & 6176.66 & $2.15 \mathrm{E}+21$ & 5693.59 & $2.35 \mathrm{E}+21$ \\
\hline & A-17 & 5932.31 & 6371.10 & $1.62 \mathrm{E}+21$ & 6053.43 & $1.99 \mathrm{E}+21$ & 5610.91 & $2.19 \mathrm{E}+21$ \\
\hline & A-18 & 5397.59 & 5842.02 & $1.46 \mathrm{E}+21$ & 5648.85 & $1.81 \mathrm{E}+21$ & 5299.91 & $1.99 \mathrm{E}+21$ \\
\hline & A-19 & 4796.53 & 5270.44 & $1.26 \mathrm{E}+21$ & 5208.16 & $1.57 \mathrm{E}+21$ & 4939.42 & $1.74 \mathrm{E}+21$ \\
\hline & A-20 & 3961.76 & 4529.31 & $1.04 \mathrm{E}+21$ & 4662.62 & $1.31 \mathrm{E}+21$ & 4459.98 & $1.46 \mathrm{E}+21$ \\
\hline
\end{tabular}


Table 24. Corrected fission power density (W/cc) and fission density (fissions/cc) for ATR Cycle 143B.

\begin{tabular}{|c|c|c|c|c|c|c|c|c|}
\hline \multirow[b]{2}{*}{$\begin{array}{l}\text { Plate } \\
\text { ID }\end{array}$} & \multirow[b]{2}{*}{ Cell } & \multirow{2}{*}{$\begin{array}{c}\text { BOC 143B } \\
\\
\text { Fission } \\
\text { Power Density } \\
(\mathrm{W} / \mathrm{cc})\end{array}$} & \multicolumn{2}{|c|}{ MOC1 143B } & \multicolumn{2}{|c|}{ MOC2 143B } & \multicolumn{2}{|c|}{ EOC 143B } \\
\hline & & & $\begin{array}{l}\text { Fission } \\
\text { Power } \\
\text { Density } \\
(\mathrm{W} / \mathrm{cc})\end{array}$ & $\begin{array}{c}\text { Fission } \\
\text { Density } \\
\text { (fissions/cc) }\end{array}$ & $\begin{array}{l}\text { Fission } \\
\text { Power } \\
\text { Density } \\
(\mathrm{W} / \mathrm{cc})\end{array}$ & $\begin{array}{c}\text { Fission } \\
\text { Density } \\
\text { (fissions/cc) }\end{array}$ & $\begin{array}{l}\text { Fission } \\
\text { Power } \\
\text { Density } \\
(\mathrm{W} / \mathrm{cc})\end{array}$ & $\begin{array}{c}\text { Fission } \\
\text { Density } \\
\text { (fissions/cc) }\end{array}$ \\
\hline \multirow{10}{*}{$1 \mathrm{~T} 2$} & A-1 & 2852.93 & 3177.81 & $1.40 \mathrm{E}+21$ & 3296.60 & $1.59 \mathrm{E}+21$ & 3390.08 & $1.78 \mathrm{E}+21$ \\
\hline & A-2 & 3290.45 & 3636.72 & $1.73 E+21$ & 3789.60 & $1.94 \mathrm{E}+21$ & 3916.24 & $2.16 \mathrm{E}+21$ \\
\hline & A-3 & 3708.32 & 3983.56 & $2.04 \mathrm{E}+21$ & 3985.56 & $2.27 \mathrm{E}+21$ & 4143.91 & $2.50 \mathrm{E}+21$ \\
\hline & A-4 & 4057.22 & 4241.49 & $2.29 \mathrm{E}+21$ & 4197.34 & $2.54 \mathrm{E}+21$ & 4407.06 & $2.78 \mathrm{E}+21$ \\
\hline & A-5 & 4212.37 & 4262.24 & $2.51 \mathrm{E}+21$ & 4147.20 & $2.76 \mathrm{E}+21$ & 4373.88 & $2.99 \mathrm{E}+21$ \\
\hline & A-6 & 4530.79 & 4429.41 & $2.67 \mathrm{E}+21$ & 4229.27 & $2.93 \mathrm{E}+21$ & 4490.36 & $3.17 \mathrm{E}+21$ \\
\hline & A-7 & 4503.88 & 4446.99 & $2.79 \mathrm{E}+21$ & 4194.27 & $3.05 \mathrm{E}+21$ & 4449.09 & $3.29 \mathrm{E}+21$ \\
\hline & A-8 & 4626.35 & 4480.25 & $2.88 \mathrm{E}+21$ & 4128.27 & $3.14 \mathrm{E}+21$ & 4413.84 & $3.37 \mathrm{E}+21$ \\
\hline & A-9 & 4693.35 & 4507.65 & $2.93 \mathrm{E}+21$ & 4066.36 & $3.19 \mathrm{E}+21$ & 4349.01 & $3.43 \mathrm{E}+21$ \\
\hline & A-10 & 4785.68 & 4463.53 & $3.01 \mathrm{E}+21$ & 4088.65 & $3.27 \mathrm{E}+21$ & 4349.33 & $3.50 \mathrm{E}+21$ \\
\hline \multirow{10}{*}{ 1B5 } & A-11 & 4621.56 & 4258.01 & $2.95 \mathrm{E}+21$ & 3893.21 & $3.19 \mathrm{E}+21$ & 4162.69 & $3.42 \mathrm{E}+21$ \\
\hline & A-12 & 4594.48 & 4313.19 & $2.88 \mathrm{E}+21$ & 3930.84 & $3.13 \mathrm{E}+21$ & 4214.86 & $3.36 \mathrm{E}+21$ \\
\hline & A-13 & 4487.63 & 4300.80 & $2.87 \mathrm{E}+21$ & 3998.98 & $3.12 \mathrm{E}+21$ & 4301.95 & $3.34 \mathrm{E}+21$ \\
\hline & A-14 & 4464.06 & 4308.20 & $2.80 \mathrm{E}+21$ & 3953.64 & $3.05 \mathrm{E}+21$ & 4231.23 & $3.28 \mathrm{E}+21$ \\
\hline & A-15 & 4374.73 & 4282.41 & $2.70 \mathrm{E}+21$ & 3989.70 & $2.95 \mathrm{E}+21$ & 4256.37 & $3.18 \mathrm{E}+21$ \\
\hline & A-16 & 4332.08 & 4171.04 & $2.58 \mathrm{E}+21$ & 4069.34 & $2.82 \mathrm{E}+21$ & 4326.80 & $3.05 \mathrm{E}+21$ \\
\hline & A-17 & 4068.60 & 4172.68 & $2.40 \mathrm{E}+21$ & 3977.59 & $2.64 \mathrm{E}+21$ & 4207.40 & $2.87 \mathrm{E}+21$ \\
\hline & A-18 & 3879.69 & 4054.96 & $2.19 \mathrm{E}+21$ & 3979.74 & $2.43 \mathrm{E}+21$ & 4179.72 & $2.65 \mathrm{E}+21$ \\
\hline & A-19 & 3614.10 & 3824.12 & $1.93 \mathrm{E}+21$ & 3797.40 & $2.15 \mathrm{E}+21$ & 3947.17 & $2.37 \mathrm{E}+21$ \\
\hline & A-20 & 3179.13 & 3419.75 & $1.63 \mathrm{E}+21$ & 3513.76 & $1.83 \mathrm{E}+21$ & 3631.25 & $2.03 \mathrm{E}+21$ \\
\hline
\end{tabular}




\subsection{Axial Gradient}

The axial gradient is represented by the neutron flux profile and the corrected fission density profile as shown in Figure 10 and Figure 11, respectively. Since the AFIP-1 experiment was irradiated in the CFT, the transverse (radial) profile is relatively flat across the plates and was not reported.

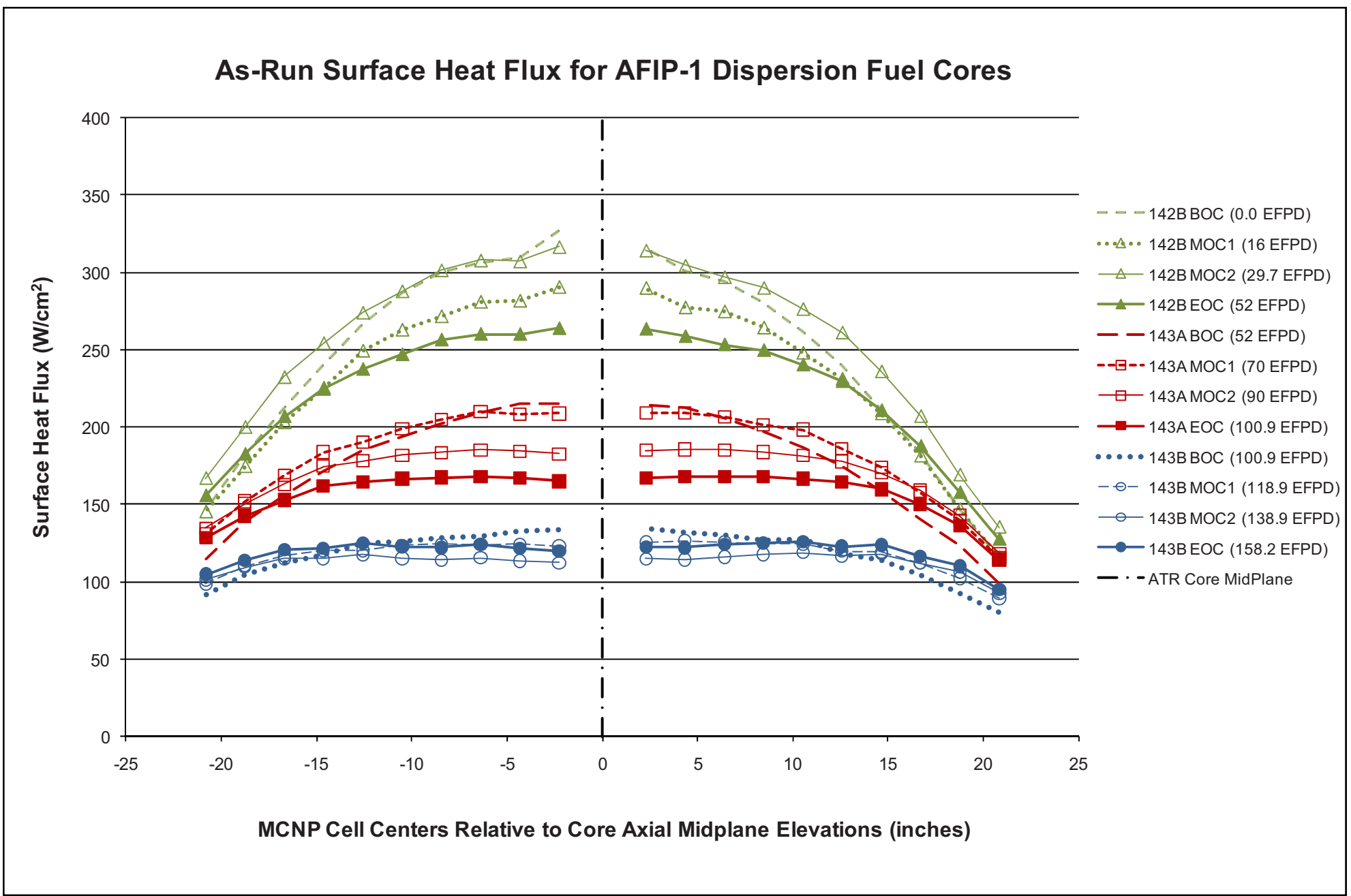

Figure 10. AFIP-1 axial neutron flux profile. 


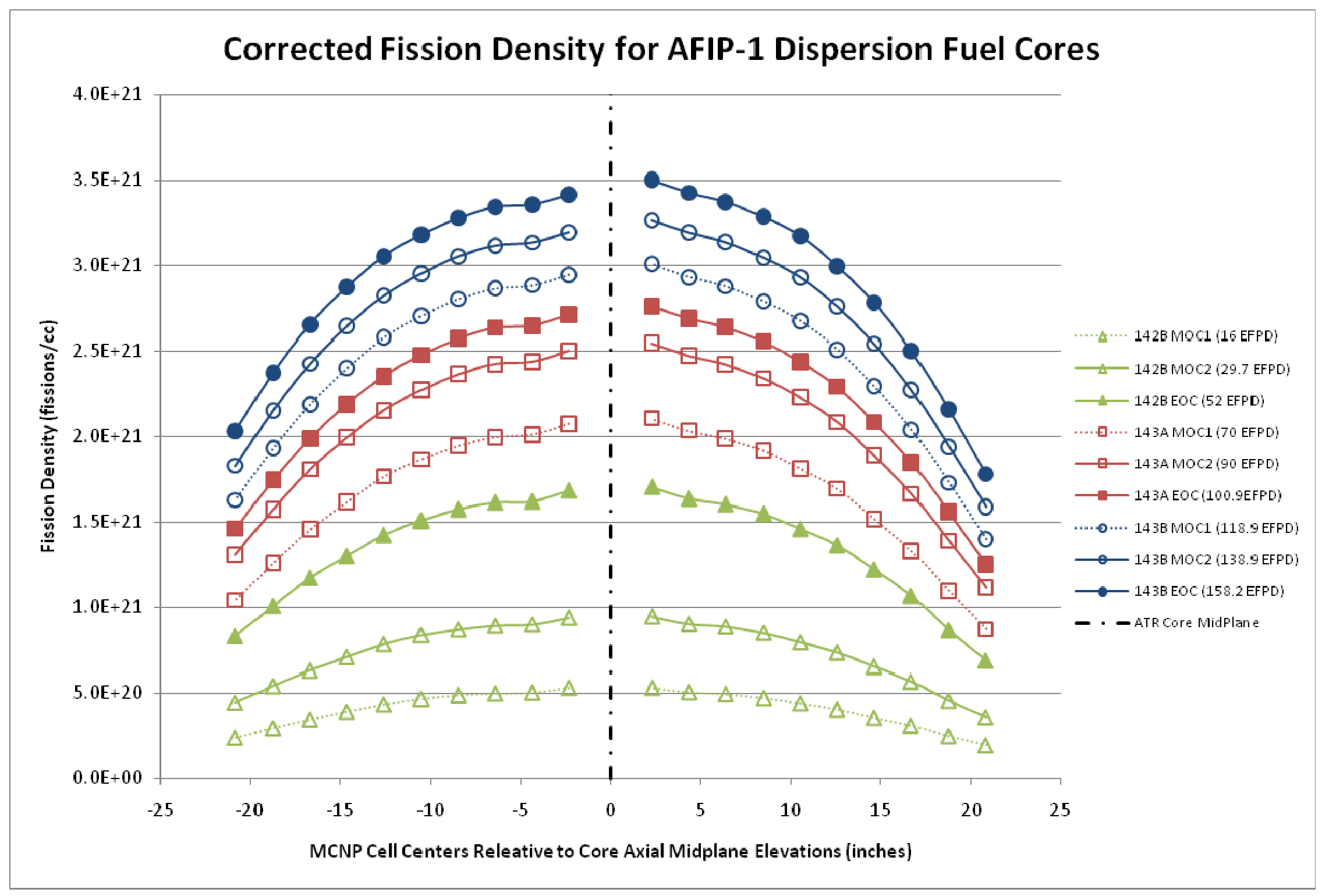

Figure 11. AFIP-1 fission density axial profile ${ }^{3}$. 


\section{HYDRAULIC TESTING}

Flow tests were performed to characterize the test assembly designed to irradiate full-size plates for the RERTR program in the ATR. The holder assembly design was used for several plate tests that were conducted in the CFT position ${ }^{6}$.

The test apparatus was designed and constructed to simulate the ATR CFT position geometry. The holder was fabricated such that the orifice plate on the bottom of the test train could be screwed on (rather than welded) to allow variation of the orifice diameter. The results of the flow tests were used to generate estimates of the coolant velocity and flow rate and are reported in Table $25^{6}$.

Table 25. AFIP irradiation vehicle flow conditions for each orifice configuration. ${ }^{6}$

\begin{tabular}{|c|c|c|c|c|}
\hline Configuration & $\begin{array}{c}\text { Total Internal } \\
\text { Loss } \\
\text { Coefficient }\end{array}$ & $\begin{array}{c}\text { Total Holder } \\
\text { Internal Flow } \\
(\mathrm{gpm})\end{array}$ & $\begin{array}{c}\text { Channel } \\
\text { Coolant Flow } \\
(\mathrm{gpm})\end{array}$ & $\begin{array}{c}\text { Channel } \\
\text { Coolant } \\
\text { Velocity }(\mathrm{m} / \mathrm{s})\end{array}$ \\
\hline $6 \mathrm{~mm}$ orifice & 0.1709 & 20.8 & 6.6 & 1.7 \\
\hline $7 \mathrm{~mm}$ orifice & 0.1203 & 24.8 & 7.9 & 2.0 \\
\hline $8 \mathrm{~mm}$ orifice & 0.0869 & 29.2 & 9.3 & 2.4 \\
\hline $8.1 \mathrm{~mm}$ orifice & 0.0861 & 29.3 & 9.3 & 2.4 \\
\hline $9 \mathrm{~mm}$ orifice & 0.0680 & 33.0 & 10.5 & 2.7 \\
\hline $10 \mathrm{~mm}$ orifice & 0.0568 & 36.1 & 11.5 & 3.0 \\
\hline Open (no orifice) & 0.00164 & 212.5 & 67.5 & 17.4 \\
\hline
\end{tabular}

Based on the results from the hydraulic testing, the orifice was sized to 0.8125 inches $(20.64 \mathrm{~mm})$ to provide a coolant channel velocity of approximately $34 \mathrm{ft} / \mathrm{s}$ for two pump operation ${ }^{7}$. 


\section{AS-RUN THERMAL ANALYSIS}

The thermal as-run analysis was performed using the as-built geometry, MCNP-calculated surface heat flux $\left(\mathrm{W} / \mathrm{cm}^{2}\right)$ and nominal coolant channel flow rate. ABAQUS ${ }^{8}$ was used to calculate the coolant channel temperatures and plate surface temperatures.

\subsection{Coolant Channel Temperature}

The coolant temperature ${ }^{9}$ was analyzed at the three flow channels in the test assembly. The left coolant channel is west of plate position B, the center coolant channel is in between plate positions A and $\mathrm{B}$, and the right coolant channel is in between plate position A and the Ram Rod (refer to Figure 1). For each cycle interval, the coolant temperature was plotted as a function of location along the test assembly with 0.0 inches being at the top of the assembly. These plots are show in Figure 12 through Figure 23 .

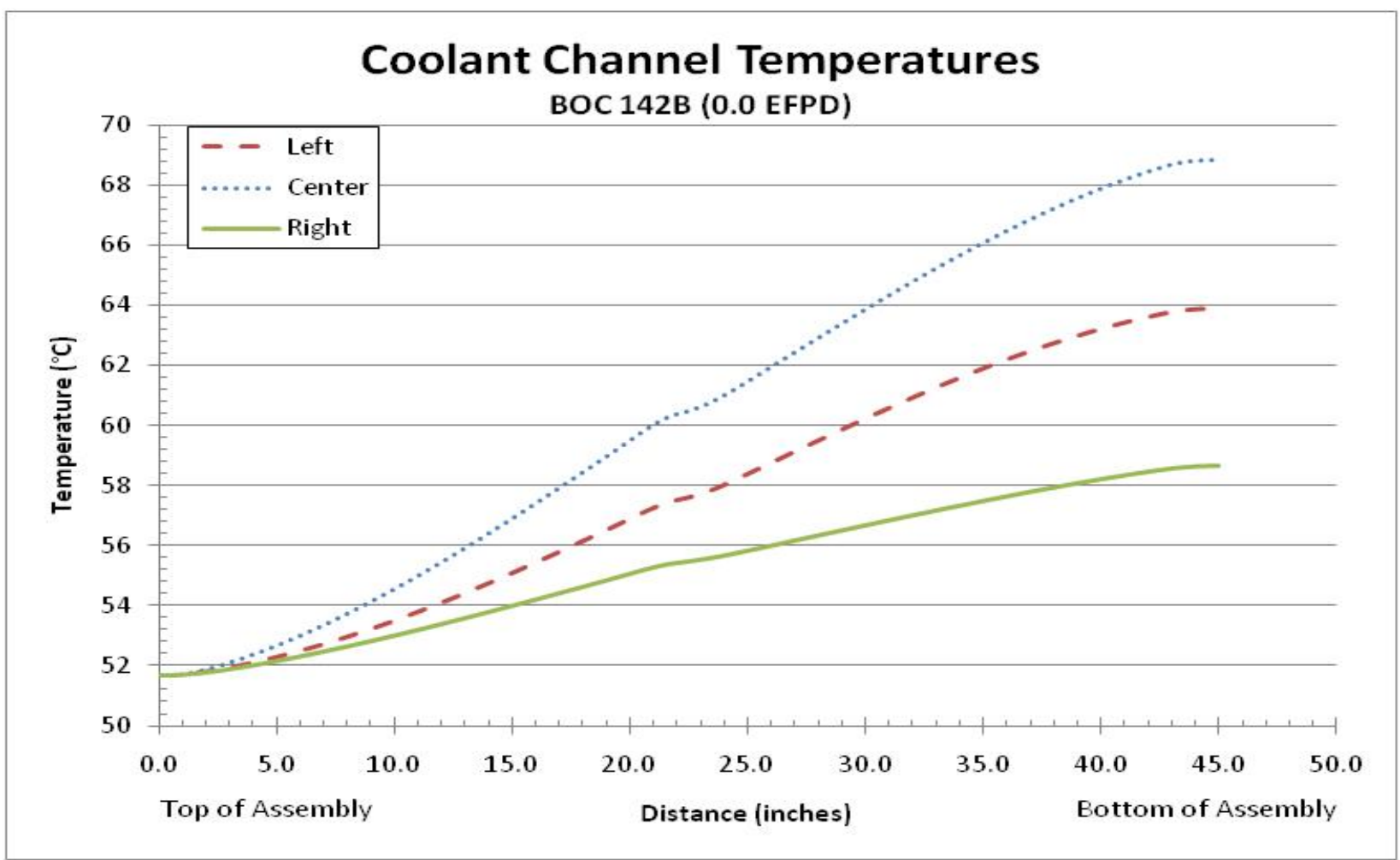

Figure 12. Coolant channel temperatures as a function of location along the test assembly at BOC 142B (0.0 EFPD). NOTE: AFIP-2 was inserted into plate position A and BOC 142B. 


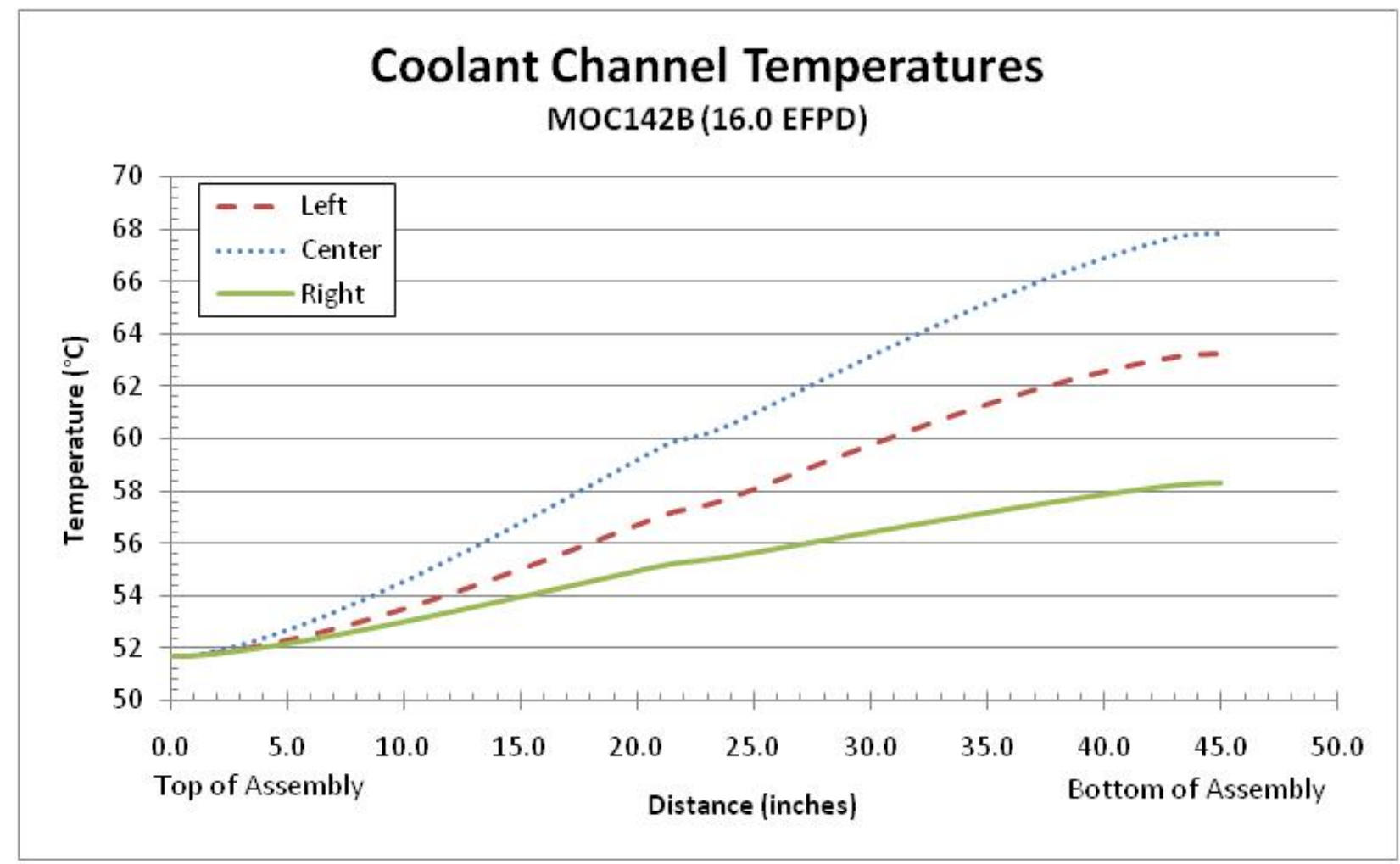

Figure 13. Coolant channel temperatures as a function of location along the test assembly at MOC 142B (16.0 EFPD, cumulative).

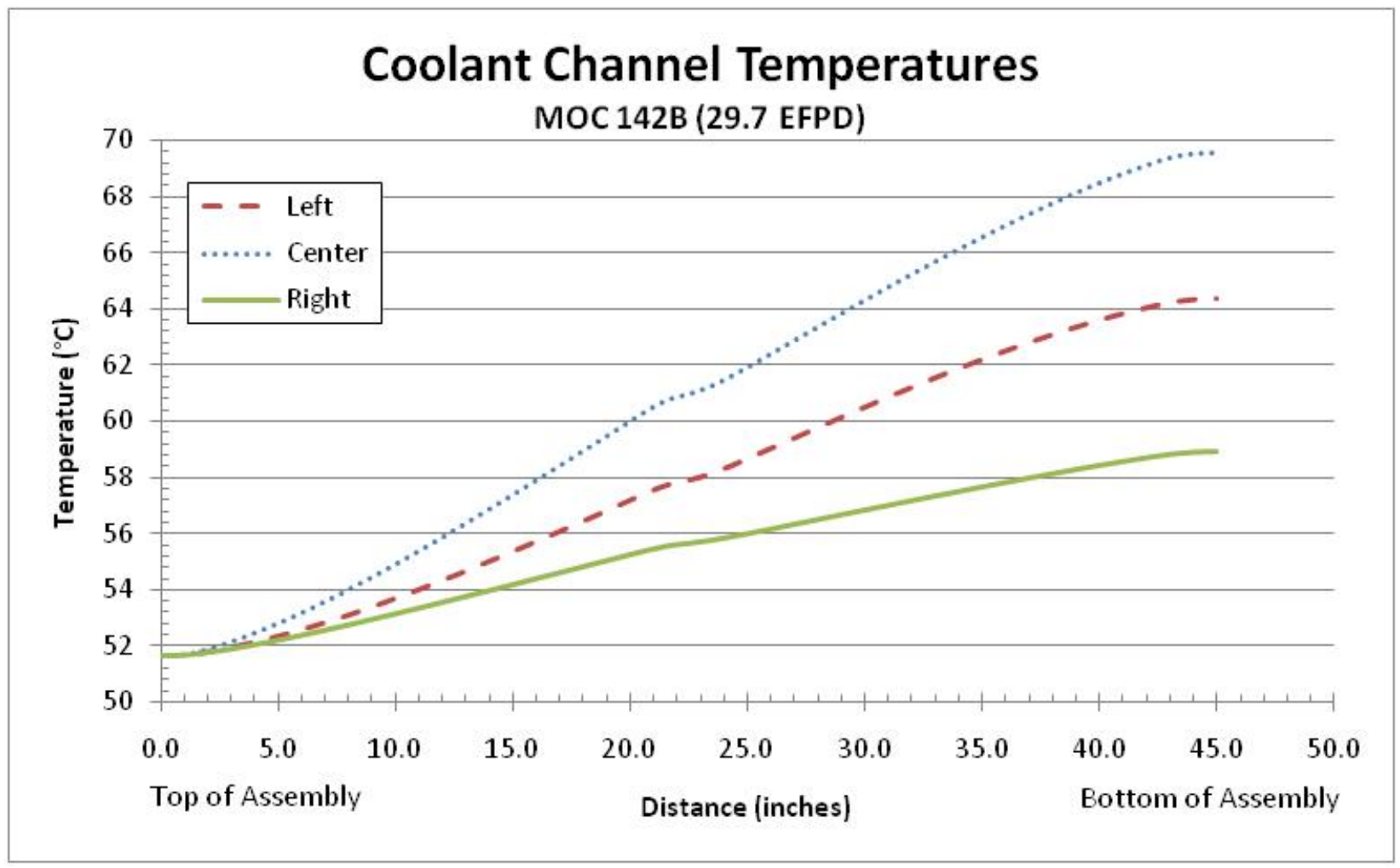

Figure 14. Coolant channel temperatures as a function of location along the test assembly at MOC 142B (29.7 EFPD cumulative). 


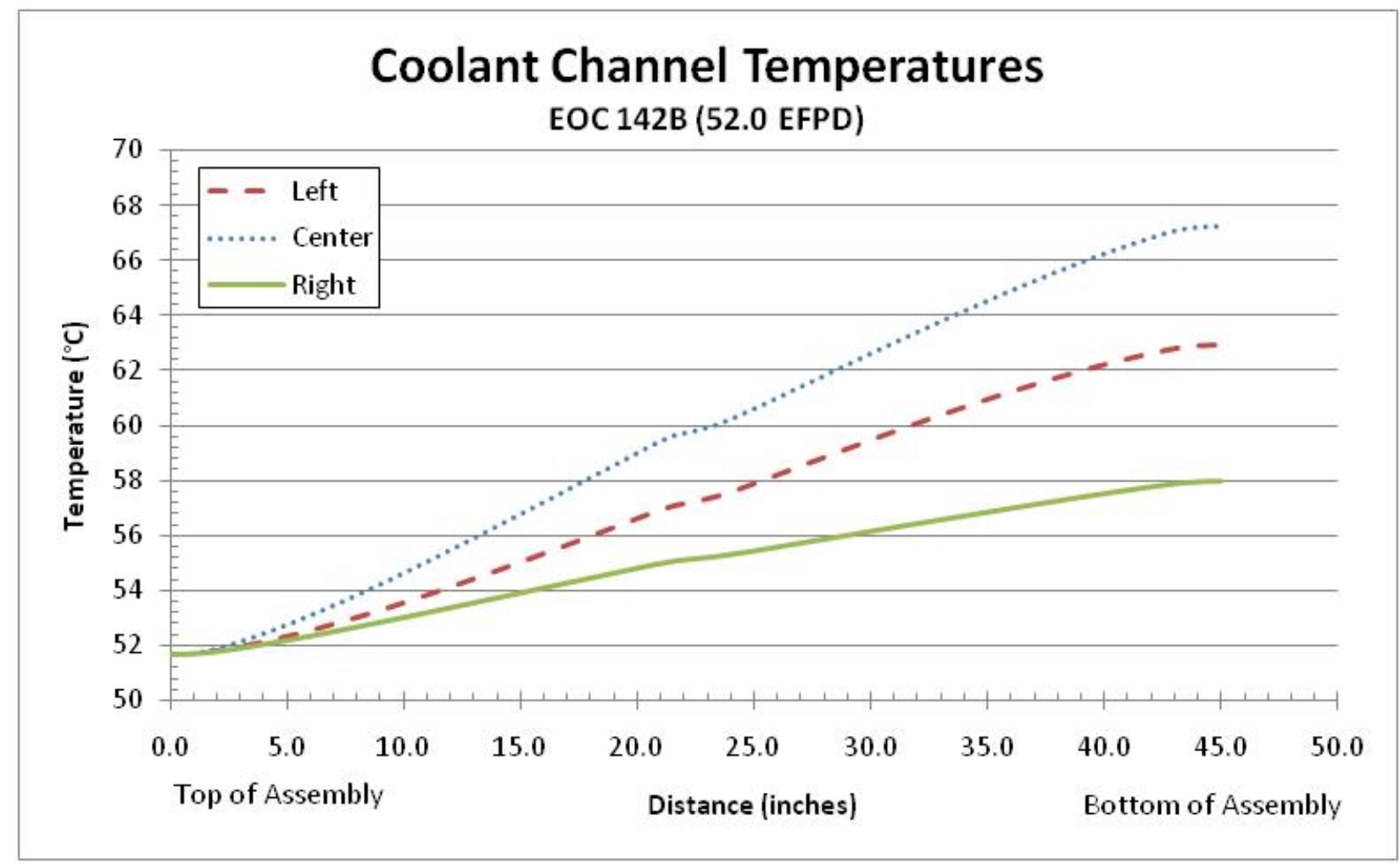

Figure 15. Coolant channel temperatures as a function of location along the test assembly at EOC 142B (52.0 EFPD cumulative).

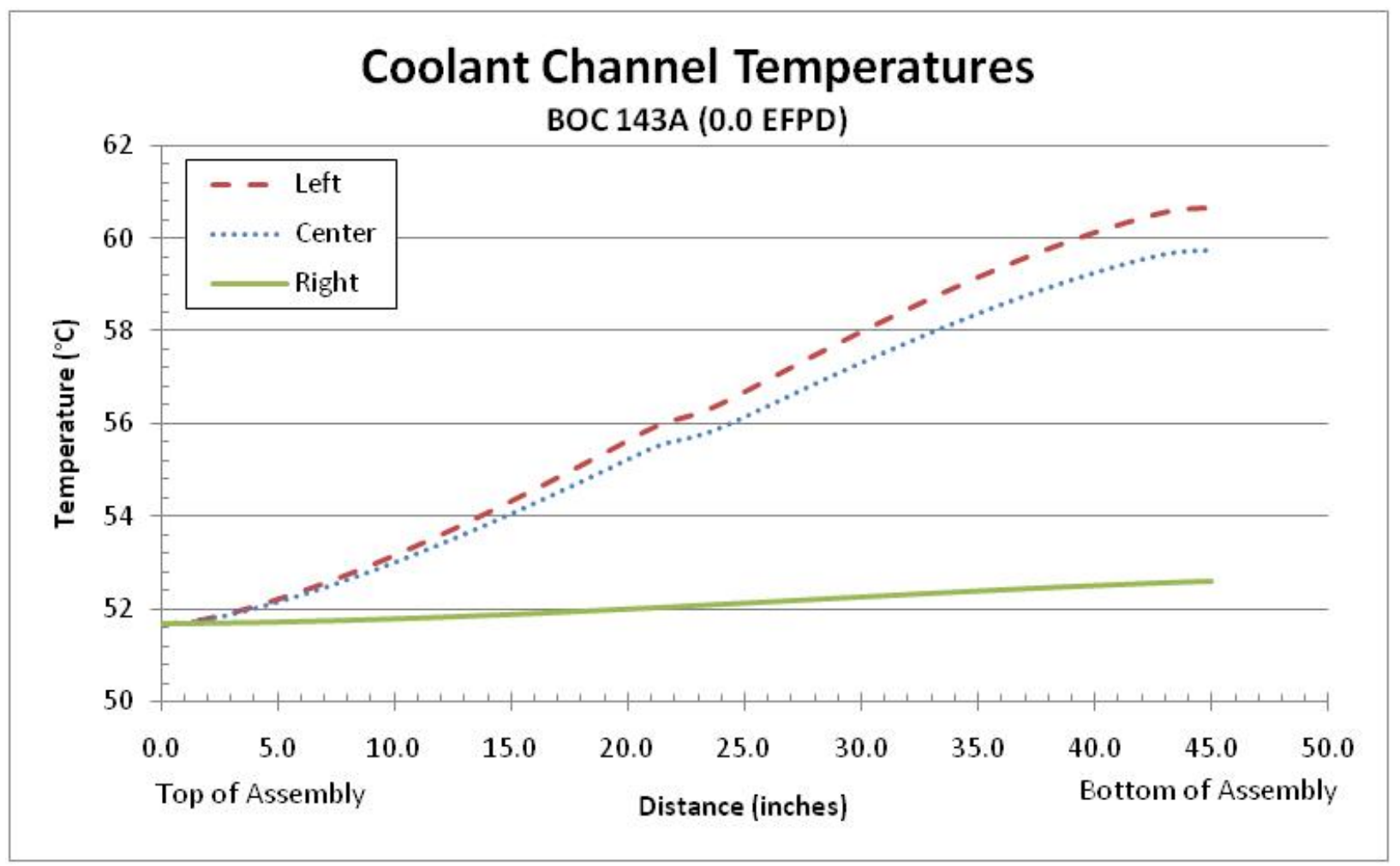

Figure 16. Coolant channel temperatures as a function of location along the test assembly at BOC 143A (52.0 EFPD cumulative). 


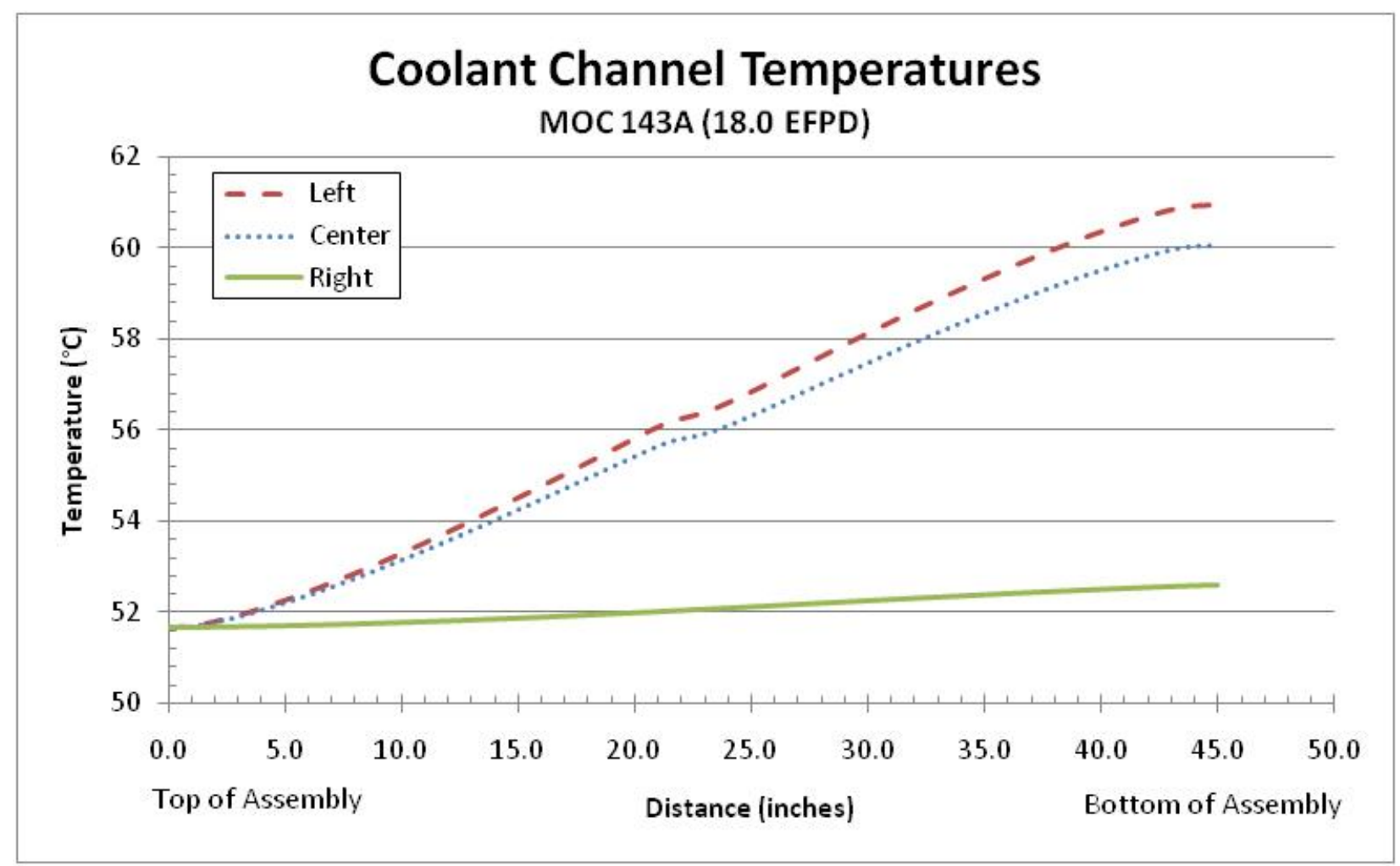

Figure 17. Coolant channel temperatures as a function of location along the test assembly at MOC 143A (70.0 EFPD cumulative).

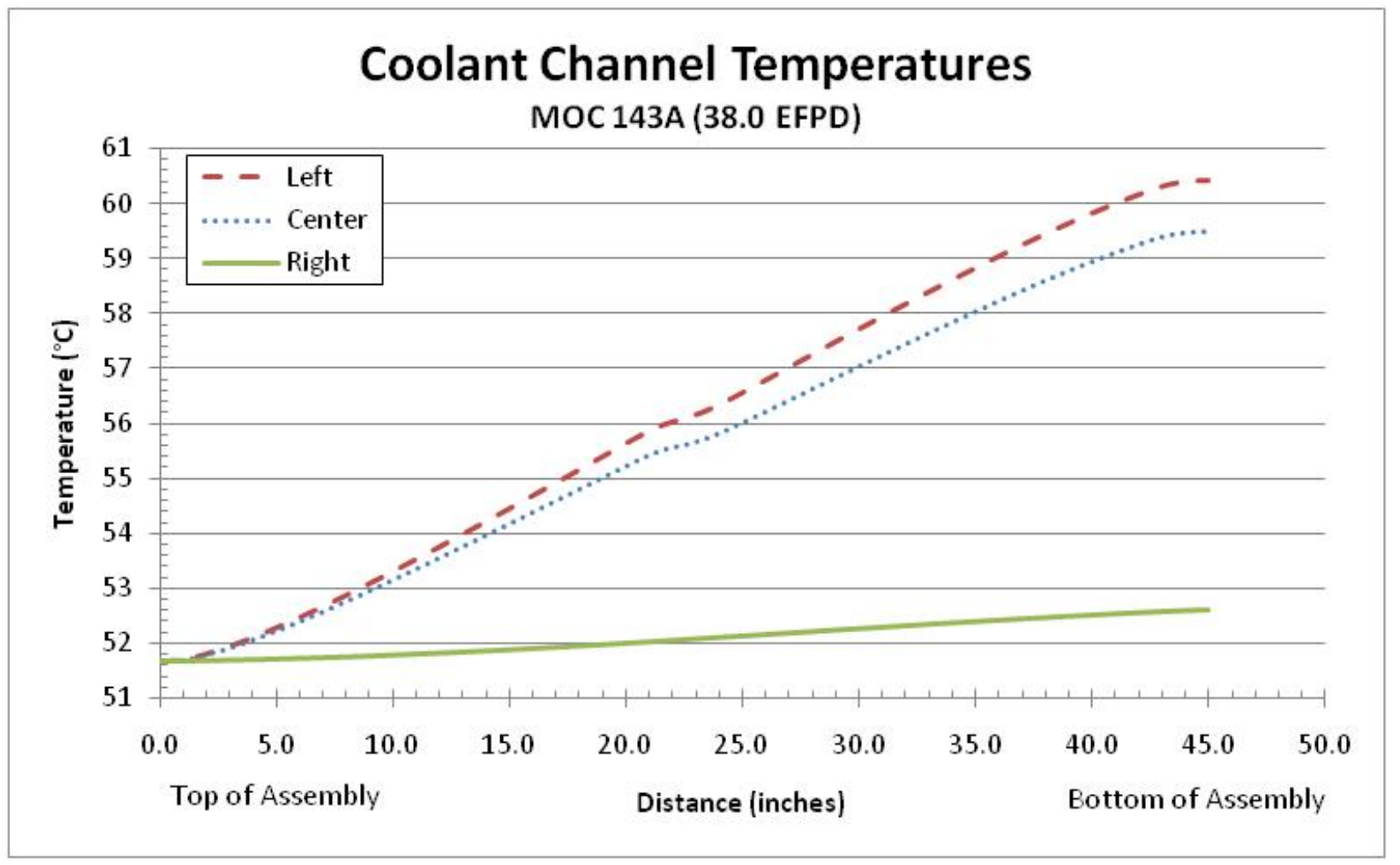

Figure 18. Coolant channel temperatures as a function of location along the test assembly at MOC 143A (90.0 EFPD cumulative). 


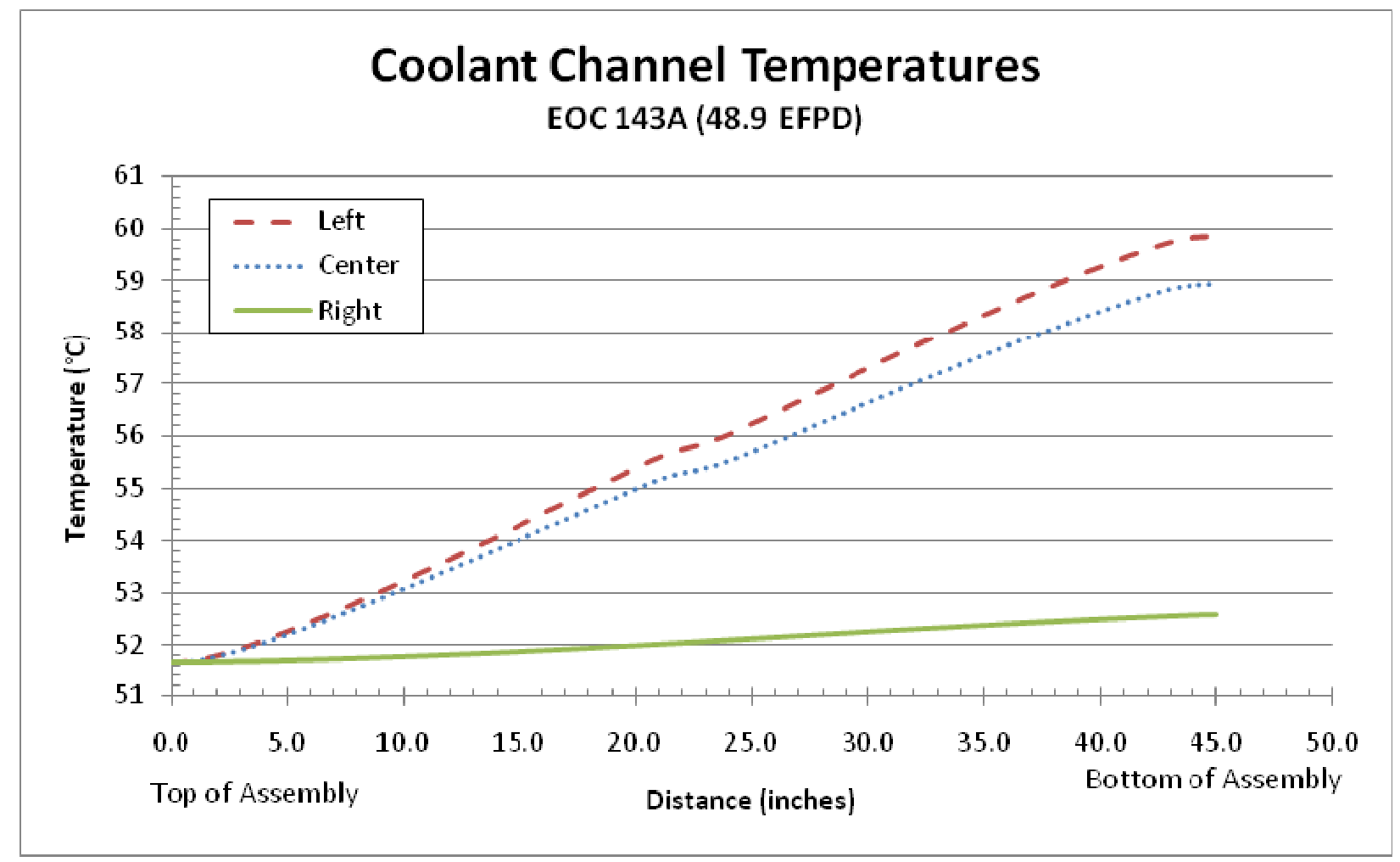

Figure 19. Coolant channel temperatures as a function of location along the test assembly at EOC 143A (100.9 EFPD cumulative).

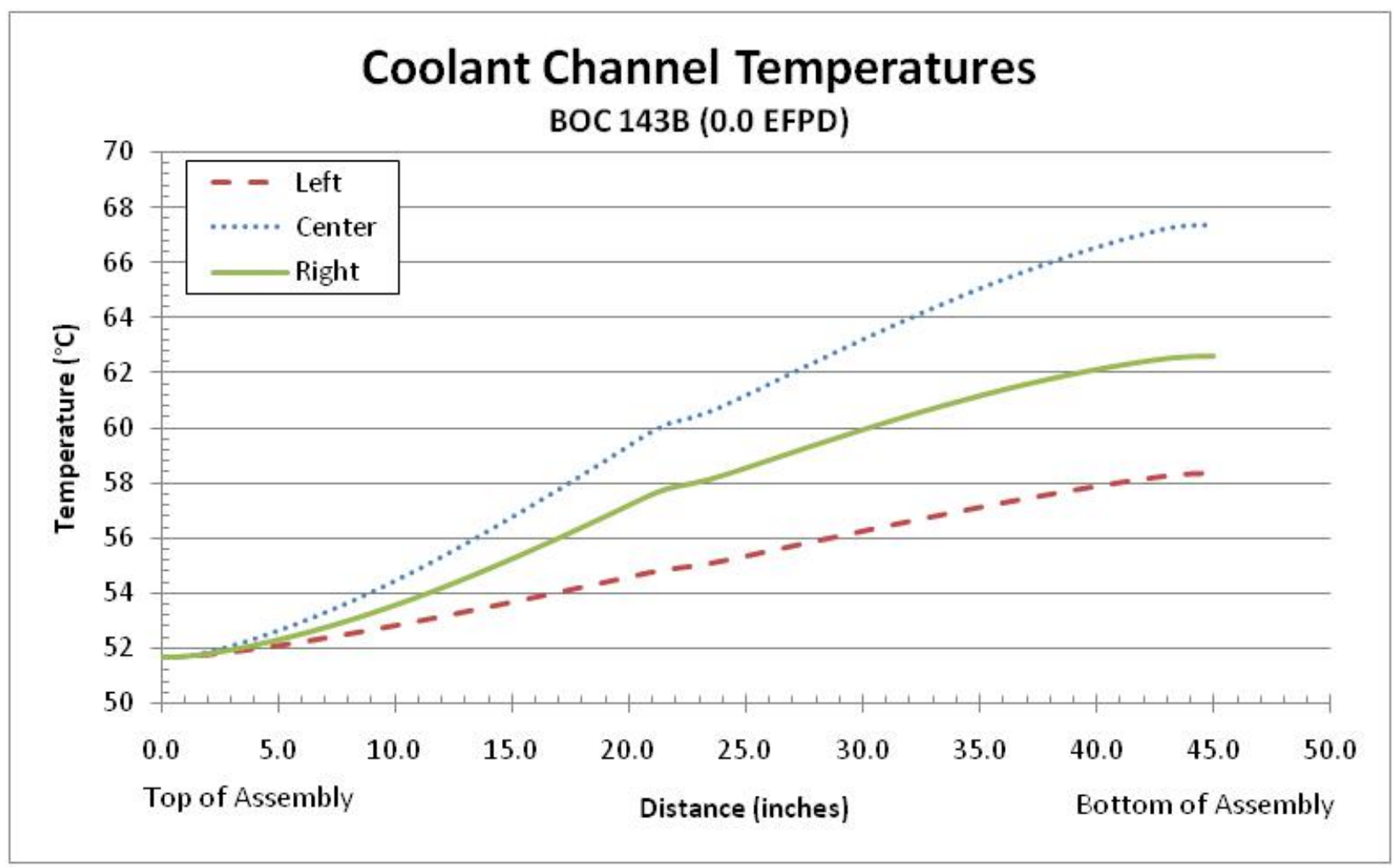

Figure 20. Coolant channel temperatures as a function of location along the test assembly at BOC 143B (100.9 EFPD cumulative). NOTE: AFIP-3 was inserted into plate position A and BOC 143B. 


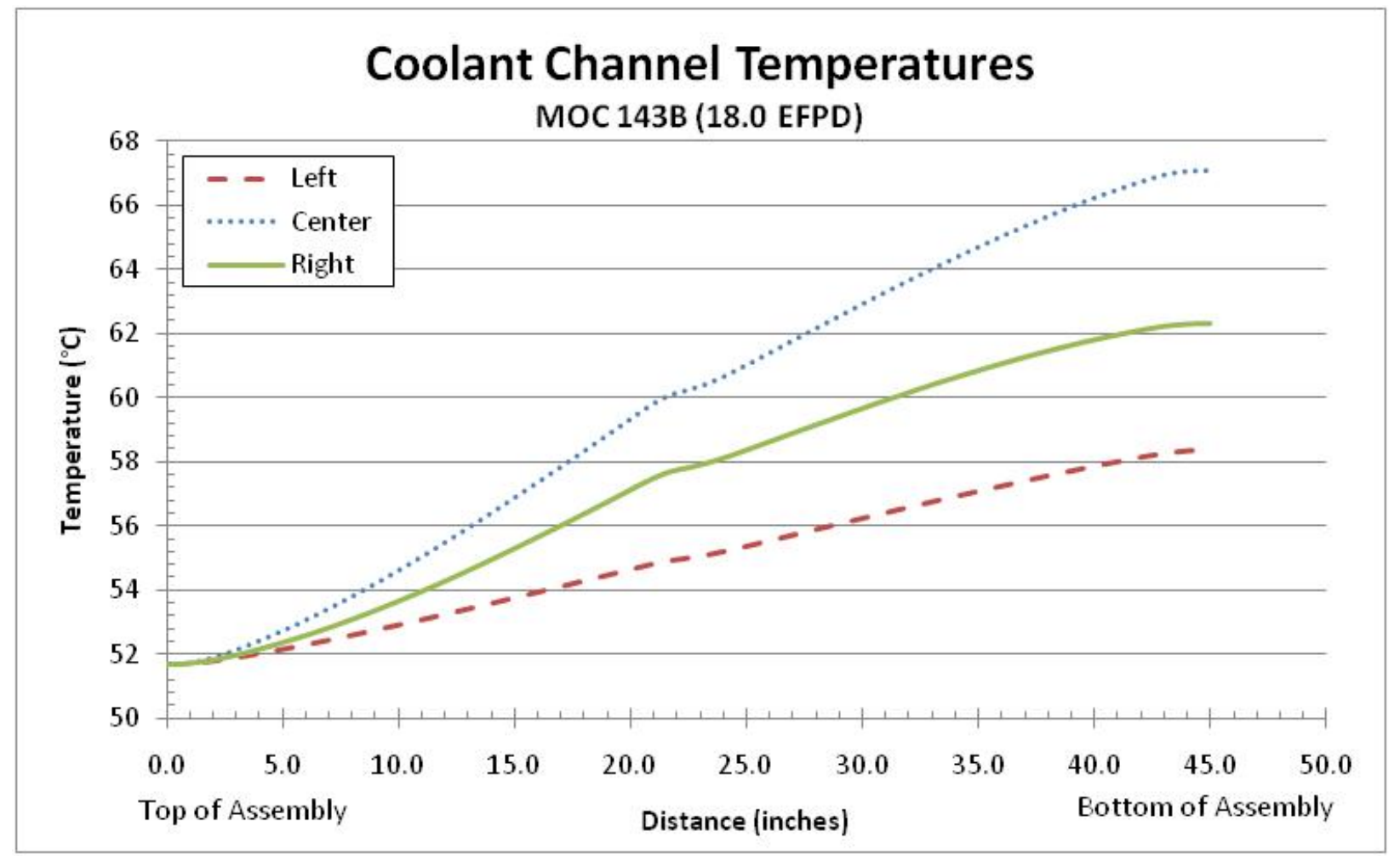

Figure 21. Coolant channel temperatures as a function of location along the test assembly at MOC 143B (118.9 EFPD cumulative).

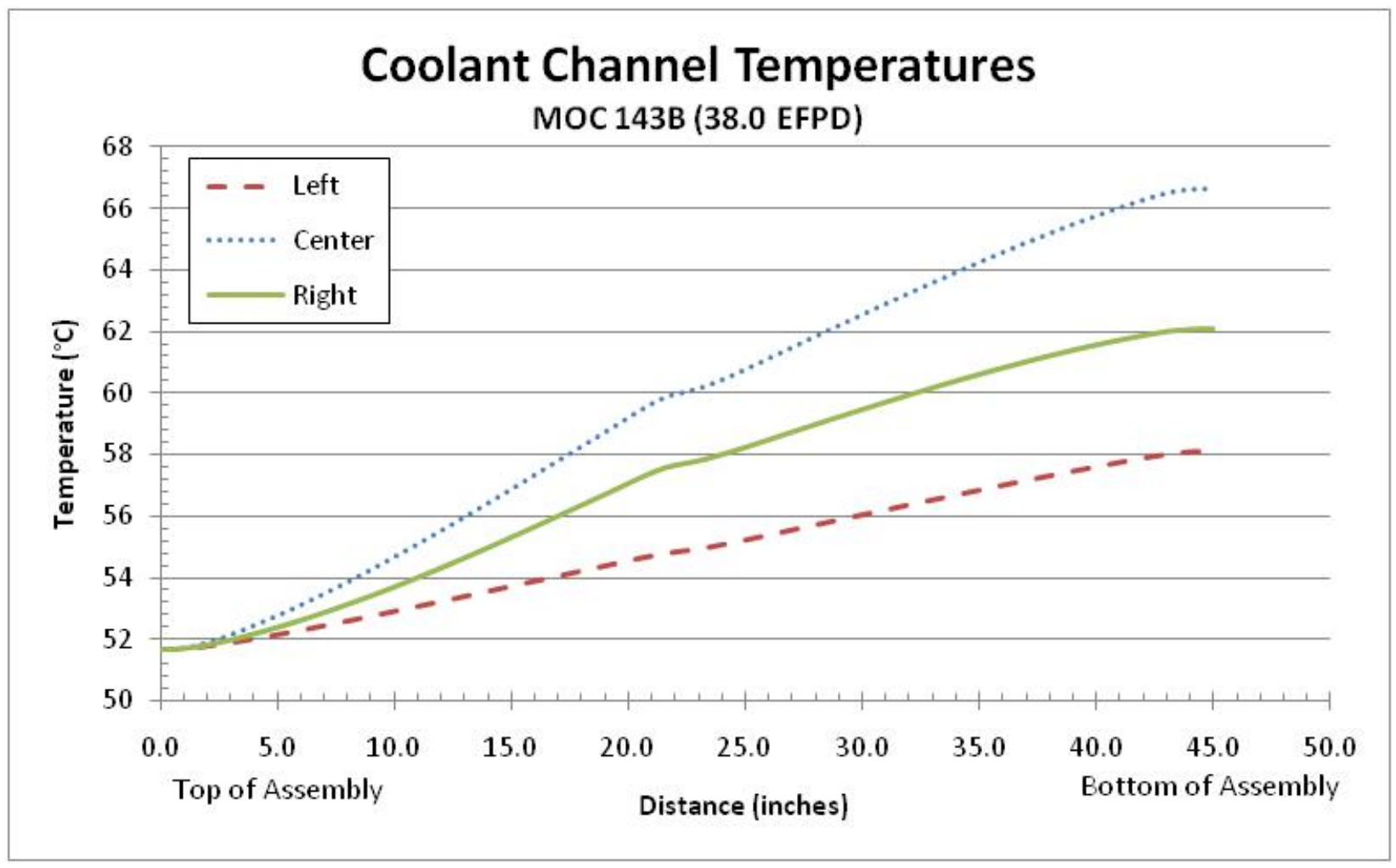

Figure 22. Coolant channel temperatures as a function of location along the test assembly at MOC 143B (138.9 EFPD cumulative). 


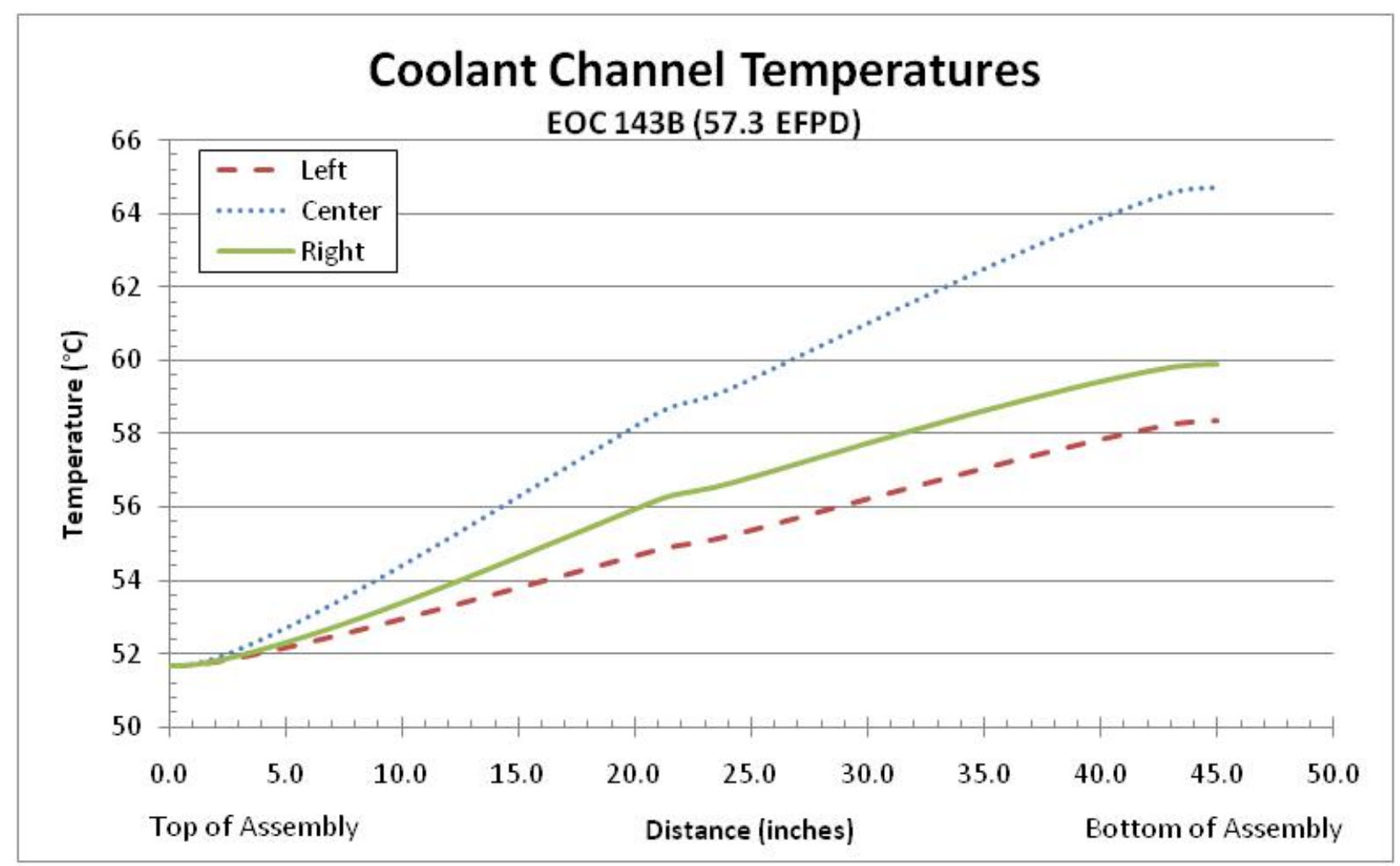

Figure 23. Coolant channel temperatures as a function of location along the test assembly at EOC 143B (158.2 EFPD cumulative). 


\subsection{Plate Surface Temperature}

The plate surface temperatures ${ }^{9}$ were analyzed at each time step for each side of the plate, with the east side of the plate facing the ram rod and west side of the plate facing plate position B. Table 26 through Table 37 tabulate the 2D map of the temperatures for each side of the plate at EOC for each cycle. The plate surface temperatures for each time step are tabulated in Appendix B.

Table 26. Temperature $\left({ }^{\circ} \mathrm{C}\right)$ map of the east side of plate 1T2 at EOC 142B (52.0 EFPD).

\begin{tabular}{|c||c|c|c|c|c|c|c|c|c|c|}
\hline \multirow{2}{*}{$\begin{array}{c}\text { Length of } \\
\text { Plate (in) }\end{array}$} & \multicolumn{10}{|c|}{ Width of Plate (inches) } \\
\hline & 0.25 & 0.50 & 0.75 & 1.00 & 1.25 & 1.50 & 1.75 & 2.00 & 2.24 \\
\hline 0.125 & 52.10 & 51.80 & 51.77 & 51.77 & 51.77 & 51.77 & 51.77 & 51.78 & 51.86 & 52.14 \\
\hline 1.00 & 53.51 & 52.46 & 52.57 & 52.58 & 52.59 & 52.59 & 52.58 & 52.52 & 52.47 & 53.36 \\
\hline 2.00 & 57.97 & 78.86 & 81.31 & 81.36 & 81.37 & 81.37 & 81.36 & 81.21 & 68.95 & 56.13 \\
\hline 3.00 & 58.44 & 79.14 & 81.62 & 81.67 & 81.68 & 81.68 & 81.67 & 81.50 & 69.26 & 56.43 \\
\hline 4.00 & 59.63 & 84.80 & 87.69 & 87.75 & 87.76 & 87.76 & 87.75 & 87.57 & 73.07 & 57.33 \\
\hline 5.00 & 60.00 & 85.04 & 87.93 & 88.00 & 88.01 & 88.01 & 87.99 & 87.81 & 73.32 & 57.58 \\
\hline 6.00 & 61.19 & 90.43 & 93.70 & 93.78 & 93.79 & 93.79 & 93.77 & 93.57 & 76.99 & 58.35 \\
\hline 7.00 & 61.54 & 90.68 & 93.95 & 94.03 & 94.05 & 94.04 & 94.02 & 93.81 & 77.26 & 58.61 \\
\hline 8.00 & 62.56 & 94.80 & 98.34 & 98.43 & 98.45 & 98.44 & 98.42 & 98.20 & 80.14 & 59.28 \\
\hline 9.00 & 62.90 & 95.05 & 98.60 & 98.70 & 98.72 & 98.71 & 98.68 & 98.45 & 80.60 & 60.52 \\
\hline 10.00 & 63.74 & 98.36 & 102.38 & 102.49 & 102.52 & 102.51 & 102.47 & 102.20 & 82.86 & 60.88 \\
\hline 11.00 & 64.08 & 98.66 & 102.69 & 102.81 & 102.84 & 102.83 & 102.78 & 102.50 & 83.06 & 60.69 \\
\hline 12.00 & 64.75 & 100.82 & 105.00 & 105.13 & 105.16 & 105.16 & 105.10 & 104.80 & 84.54 & 60.98 \\
\hline 13.00 & 65.08 & 101.13 & 105.31 & 105.45 & 105.48 & 105.48 & 105.41 & 105.11 & 84.85 & 61.34 \\
\hline 14.00 & 65.62 & 103.01 & 107.32 & 107.46 & 107.50 & 107.50 & 107.43 & 107.11 & 86.14 & 61.83 \\
\hline 15.00 & 65.95 & 103.32 & 107.64 & 107.79 & 107.82 & 107.82 & 107.75 & 107.42 & 86.42 & 62.02 \\
\hline 16.00 & 66.39 & 104.27 & 108.64 & 108.79 & 108.83 & 108.83 & 108.75 & 108.41 & 87.12 & 62.24 \\
\hline 17.00 & 66.69 & 104.58 & 108.95 & 109.11 & 109.16 & 109.15 & 109.06 & 108.72 & 87.53 & 63.37 \\
\hline 18.00 & 67.11 & 105.77 & 110.21 & 110.38 & 110.43 & 110.42 & 110.33 & 109.98 & 88.50 & 64.05 \\
\hline 19.00 & 67.42 & 106.09 & 110.53 & 110.71 & 110.75 & 110.75 & 110.65 & 110.29 & 88.68 & 64.10 \\
\hline 20.00 & 67.84 & 107.22 & 111.73 & 111.91 & 111.96 & 111.96 & 111.85 & 111.48 & 89.39 & 63.76 \\
\hline 21.00 & 68.09 & 107.50 & 112.01 & 112.20 & 112.25 & 112.25 & 112.14 & 111.76 & 89.64 & 63.73 \\
\hline 22.00 & 62.00 & 64.89 & 66.21 & 66.38 & 66.44 & 66.43 & 66.30 & 65.86 & 62.76 & 60.06 \\
\hline 22.50 & 60.00 & 59.28 & 59.46 & 59.60 & 59.66 & 59.65 & 59.52 & 59.30 & 59.27 & 59.62 \\
\hline
\end{tabular}


Table 27. Temperature $\left({ }^{\circ} \mathrm{C}\right)$ map of the west side of plate $1 \mathrm{~T} 2$ at EOC 142B (52.0 EFPD).

\begin{tabular}{|c|c|c|c|c|c|c|c|c|c|c|}
\hline \multirow{2}{*}{$\begin{array}{c}\text { Length of } \\
\text { Plate (in) }\end{array}$} & \multicolumn{10}{|c|}{ Width of Plate (inches) } \\
\hline & 0.05 & 0.25 & 0.50 & 0.75 & 1.00 & 1.25 & 1.50 & 1.75 & 2.00 & 2.24 \\
\hline 0.125 & 52.36 & 51.80 & 51.77 & 51.77 & 51.77 & 51.77 & 51.77 & 51.78 & 51.85 & 52.34 \\
\hline 1.00 & 53.87 & 52.46 & 52.57 & 52.58 & 52.58 & 52.58 & 52.58 & 52.52 & 52.47 & 53.62 \\
\hline 2.00 & 57.76 & 78.83 & 81.29 & 81.34 & 81.35 & 81.35 & 81.34 & 81.19 & 68.93 & 55.66 \\
\hline 3.00 & 58.27 & 79.09 & 81.56 & 81.61 & 81.62 & 81.62 & 81.61 & 81.45 & 69.22 & 55.77 \\
\hline 4.00 & 59.39 & 84.71 & 87.59 & 87.66 & 87.66 & 87.66 & 87.65 & 87.48 & 72.99 & 56.60 \\
\hline 5.00 & 59.79 & 84.90 & 87.79 & 87.86 & 87.86 & 87.86 & 87.85 & 87.67 & 73.20 & 56.77 \\
\hline 6.00 & 60.93 & 90.25 & 93.52 & 93.59 & 93.60 & 93.59 & 93.58 & 93.38 & 76.83 & 57.45 \\
\hline 7.00 & 61.35 & 90.48 & 93.74 & 93.82 & 93.83 & 93.83 & 93.81 & 93.60 & 77.07 & 57.76 \\
\hline 8.00 & 62.31 & 94.51 & 98.05 & 98.13 & 98.14 & 98.14 & 98.12 & 97.90 & 79.87 & 58.35 \\
\hline 9.00 & 62.68 & 94.71 & 98.25 & 98.33 & 98.34 & 98.34 & 98.32 & 98.10 & 80.28 & 60.57 \\
\hline 10.00 & 63.51 & 97.98 & 101.91 & 102.02 & 102.03 & 102.03 & 102.00 & 101.73 & 82.50 & 60.65 \\
\hline 11.00 & 63.87 & 98.19 & 102.16 & 102.27 & 102.28 & 102.28 & 102.24 & 101.97 & 82.64 & 59.90 \\
\hline 12.00 & 64.53 & 100.24 & 104.40 & 104.51 & 104.54 & 104.53 & 104.49 & 104.20 & 84.07 & 59.78 \\
\hline 13.00 & 64.87 & 100.48 & 104.65 & 104.77 & 104.79 & 104.79 & 104.74 & 104.44 & 84.34 & 60.08 \\
\hline 14.00 & 65.43 & 102.30 & 106.60 & 106.72 & 106.75 & 106.74 & 106.69 & 106.38 & 85.58 & 60.66 \\
\hline 15.00 & 65.76 & 102.55 & 106.85 & 106.98 & 107.01 & 107.00 & 106.94 & 106.63 & 85.80 & 60.91 \\
\hline 16.00 & 66.19 & 103.44 & 107.78 & 107.91 & 107.94 & 107.94 & 107.88 & 107.55 & 86.45 & 61.10 \\
\hline 17.00 & 66.50 & 103.68 & 108.03 & 108.17 & 108.20 & 108.19 & 108.13 & 107.80 & 86.80 & 63.14 \\
\hline 18.00 & 66.91 & 104.82 & 109.23 & 109.38 & 109.41 & 109.40 & 109.33 & 108.99 & 87.72 & 64.12 \\
\hline 19.00 & 67.22 & 105.07 & 109.49 & 109.64 & 109.67 & 109.67 & 109.59 & 109.24 & 87.86 & 64.00 \\
\hline 20.00 & 67.63 & 106.14 & 110.62 & 110.77 & 110.81 & 110.80 & 110.72 & 110.37 & 88.52 & 62.82 \\
\hline 21.00 & 67.85 & 106.37 & 110.84 & 111.00 & 111.04 & 111.03 & 110.95 & 110.59 & 88.72 & 62.37 \\
\hline 22.00 & 62.42 & 64.01 & 65.30 & 65.44 & 65.49 & 65.49 & 65.37 & 64.95 & 61.86 & 59.36 \\
\hline 22.50 & 60.75 & 58.36 & 58.51 & 58.62 & 58.68 & 58.67 & 58.55 & 58.35 & 58.32 & 60.58 \\
\hline
\end{tabular}


Table 28. Temperature $\left({ }^{\circ} \mathrm{C}\right)$ map of the east side of plate 1B5 at EOC 142B (52.0 EFPD).

\begin{tabular}{|c||c|c|c|c|c|c|c|c|c|c|}
\hline \multirow{2}{*}{$\begin{array}{l}\text { Length of } \\
\text { Plate (in) }\end{array}$} & \multicolumn{10}{|c|}{ Width of Plate (inches) } \\
\hline 22.625 & 60.10 & 59.34 & 59.53 & 59.67 & 59.74 & 59.73 & 59.59 & 59.37 & 59.29 & 59.44 \\
\hline 23.00 & 62.04 & 65.00 & 66.30 & 66.47 & 66.53 & 66.52 & 66.38 & 65.96 & 62.87 & 60.11 \\
\hline 24.00 & 68.29 & 108.08 & 112.56 & 112.76 & 112.81 & 112.81 & 112.69 & 112.32 & 90.15 & 63.94 \\
\hline 25.00 & 68.56 & 108.41 & 112.90 & 113.10 & 113.16 & 113.16 & 113.03 & 112.65 & 90.47 & 64.41 \\
\hline 26.00 & 68.73 & 108.14 & 112.60 & 112.80 & 112.86 & 112.86 & 112.73 & 112.35 & 90.51 & 65.31 \\
\hline 27.00 & 68.95 & 108.43 & 112.90 & 113.10 & 113.17 & 113.16 & 113.03 & 112.64 & 90.90 & 65.75 \\
\hline 28.00 & 69.16 & 108.73 & 113.20 & 113.41 & 113.47 & 113.47 & 113.33 & 112.94 & 91.04 & 65.57 \\
\hline 29.00 & 69.36 & 109.04 & 113.52 & 113.73 & 113.80 & 113.79 & 113.65 & 113.25 & 91.22 & 65.16 \\
\hline 30.00 & 69.46 & 108.71 & 113.15 & 113.36 & 113.43 & 113.43 & 113.28 & 112.88 & 91.09 & 65.07 \\
\hline 31.00 & 69.62 & 108.98 & 113.42 & 113.64 & 113.71 & 113.70 & 113.56 & 113.15 & 91.38 & 65.29 \\
\hline 32.00 & 69.54 & 107.70 & 112.03 & 112.25 & 112.32 & 112.31 & 112.16 & 111.75 & 90.63 & 65.18 \\
\hline 33.00 & 69.69 & 107.96 & 112.29 & 112.51 & 112.59 & 112.58 & 112.42 & 112.01 & 90.86 & 65.22 \\
\hline 34.00 & 69.61 & 106.70 & 110.90 & 111.13 & 111.21 & 111.20 & 111.04 & 110.62 & 90.13 & 65.46 \\
\hline 35.00 & 69.73 & 106.95 & 111.16 & 111.39 & 111.47 & 111.46 & 111.30 & 110.87 & 90.49 & 66.17 \\
\hline 36.00 & 69.52 & 105.06 & 109.10 & 109.33 & 109.41 & 109.40 & 109.24 & 108.81 & 89.47 & 66.45 \\
\hline 37.00 & 69.61 & 105.29 & 109.34 & 109.58 & 109.66 & 109.65 & 109.48 & 109.05 & 89.56 & 66.16 \\
\hline 38.00 & 69.26 & 102.62 & 106.44 & 106.68 & 106.76 & 106.75 & 106.57 & 106.15 & 87.82 & 65.35 \\
\hline 39.00 & 69.32 & 102.84 & 106.66 & 106.90 & 106.98 & 106.97 & 106.79 & 106.37 & 88.00 & 65.19 \\
\hline 40.00 & 68.75 & 98.95 & 102.44 & 102.68 & 102.77 & 102.76 & 102.57 & 102.15 & 85.63 & 64.94 \\
\hline 41.00 & 68.76 & 99.14 & 102.63 & 102.87 & 102.96 & 102.95 & 102.76 & 102.34 & 85.80 & 65.04 \\
\hline 42.00 & 68.06 & 94.74 & 97.84 & 98.08 & 98.17 & 98.16 & 97.97 & 97.55 & 83.04 & 64.40 \\
\hline 43.00 & 68.02 & 94.89 & 97.99 & 98.23 & 98.32 & 98.31 & 98.12 & 97.71 & 83.18 & 64.58 \\
\hline 44.00 & 63.63 & 66.04 & 66.62 & 66.85 & 66.95 & 66.93 & 66.71 & 66.33 & 65.34 & 62.21 \\
\hline 45.00 & 62.83 & 65.46 & 65.87 & 66.09 & 66.18 & 66.17 & 65.95 & 65.64 & 64.97 & 61.81 \\
\hline
\end{tabular}


Table 29. Temperature $\left({ }^{\circ} \mathrm{C}\right)$ map of the west side of plate $1 \mathrm{~B} 5$ at EOC $142 \mathrm{~B}(52.0 \mathrm{EFPD})$.

\begin{tabular}{|c||c|c|c|c|c|c|c|c|c|c|}
\hline \multirow{2}{*}{$\begin{array}{c}\text { Length of } \\
\text { Plate (in) }\end{array}$} & \multicolumn{10}{|c|}{ Width of Plate (inches) } \\
\hline 22.625 & 60.69 & 58.43 & 58.58 & 58.70 & 58.75 & 58.74 & 58.62 & 58.42 & 58.34 & 59.93 \\
\hline 23.00 & 62.52 & 64.09 & 65.36 & 65.51 & 65.56 & 65.55 & 65.43 & 65.02 & 61.95 & 59.45 \\
\hline 24.00 & 68.24 & 106.83 & 111.29 & 111.45 & 111.50 & 111.49 & 111.39 & 111.04 & 89.15 & 62.69 \\
\hline 25.00 & 68.50 & 107.13 & 111.59 & 111.76 & 111.80 & 111.80 & 111.69 & 111.33 & 89.43 & 63.54 \\
\hline 26.00 & 68.64 & 106.82 & 111.25 & 111.42 & 111.47 & 111.46 & 111.35 & 110.98 & 89.43 & 65.27 \\
\hline 27.00 & 68.82 & 107.07 & 111.50 & 111.68 & 111.73 & 111.72 & 111.61 & 111.23 & 89.78 & 65.89 \\
\hline 28.00 & 69.00 & 107.33 & 111.77 & 111.94 & 111.99 & 111.98 & 111.87 & 111.49 & 89.90 & 65.35 \\
\hline 29.00 & 69.18 & 107.59 & 112.03 & 112.21 & 112.26 & 112.25 & 112.13 & 111.75 & 90.03 & 64.20 \\
\hline 30.00 & 69.24 & 107.22 & 111.62 & 111.80 & 111.85 & 111.84 & 111.72 & 111.33 & 89.86 & 63.78 \\
\hline 31.00 & 69.36 & 107.45 & 111.85 & 112.03 & 112.09 & 112.07 & 111.95 & 111.55 & 90.09 & 63.91 \\
\hline 32.00 & 69.24 & 106.12 & 110.40 & 110.59 & 110.64 & 110.63 & 110.50 & 110.10 & 89.31 & 63.65 \\
\hline 33.00 & 69.35 & 106.36 & 110.64 & 110.83 & 110.89 & 110.88 & 110.74 & 110.34 & 89.50 & 63.81 \\
\hline 34.00 & 69.22 & 105.01 & 109.17 & 109.37 & 109.42 & 109.41 & 109.27 & 108.86 & 88.72 & 64.40 \\
\hline 35.00 & 69.29 & 105.21 & 109.38 & 109.58 & 109.64 & 109.62 & 109.48 & 109.07 & 89.01 & 65.79 \\
\hline 36.00 & 69.03 & 103.26 & 107.26 & 107.46 & 107.52 & 107.51 & 107.36 & 106.95 & 87.96 & 66.43 \\
\hline 37.00 & 69.07 & 103.46 & 107.46 & 107.66 & 107.72 & 107.71 & 107.55 & 107.14 & 88.01 & 65.78 \\
\hline 38.00 & 68.67 & 100.71 & 104.48 & 104.68 & 104.75 & 104.73 & 104.57 & 104.16 & 86.24 & 64.23 \\
\hline 39.00 & 68.67 & 100.88 & 104.66 & 104.86 & 104.92 & 104.91 & 104.75 & 104.34 & 86.38 & 63.71 \\
\hline 40.00 & 68.08 & 96.92 & 100.36 & 100.57 & 100.63 & 100.61 & 100.45 & 100.04 & 83.95 & 63.43 \\
\hline 41.00 & 68.02 & 97.06 & 100.50 & 100.71 & 100.77 & 100.75 & 100.59 & 100.18 & 84.08 & 63.63 \\
\hline 42.00 & 67.29 & 92.58 & 95.63 & 95.83 & 95.90 & 95.88 & 95.71 & 95.31 & 81.26 & 62.93 \\
\hline 43.00 & 67.18 & 92.69 & 95.74 & 95.94 & 96.01 & 95.99 & 95.82 & 95.42 & 81.36 & 63.42 \\
\hline 44.00 & 63.22 & 64.39 & 64.93 & 65.13 & 65.21 & 65.19 & 64.99 & 64.62 & 63.66 & 61.93 \\
\hline 45.00 & 62.47 & 63.81 & 64.17 & 64.36 & 64.44 & 64.41 & 64.21 & 63.92 & 63.27 & 61.71 \\
\hline
\end{tabular}


Table 30. Temperature $\left({ }^{\circ} \mathrm{C}\right)$ map of the east side of plate 1T2 at EOC 143A (100.9 EFPD).

\begin{tabular}{|c||c|c|c|c|c|c|c|c|c|c|}
\hline \multirow{2}{*}{$\begin{array}{c}\text { Length of } \\
\text { Plate (in) }\end{array}$} & \multicolumn{10}{|c|}{ Width of Plate (inches) } \\
\hline 0.125 & 52.08 & 51.79 & 51.77 & 51.77 & 51.77 & 51.77 & 51.77 & 51.77 & 51.85 & 52.12 \\
\hline 1.00 & 53.17 & 52.37 & 52.48 & 52.49 & 52.49 & 52.49 & 52.49 & 52.43 & 52.37 & 53.07 \\
\hline 2.00 & 56.33 & 76.26 & 78.55 & 78.59 & 78.60 & 78.60 & 78.59 & 78.45 & 67.12 & 54.88 \\
\hline 3.00 & 56.68 & 76.46 & 78.77 & 78.82 & 78.82 & 78.82 & 78.82 & 78.66 & 67.35 & 55.08 \\
\hline 4.00 & 57.48 & 80.71 & 83.34 & 83.39 & 83.40 & 83.39 & 83.39 & 83.22 & 70.17 & 55.70 \\
\hline 5.00 & 57.74 & 80.85 & 83.49 & 83.54 & 83.55 & 83.55 & 83.54 & 83.37 & 70.33 & 55.88 \\
\hline 6.00 & 58.34 & 83.42 & 86.24 & 86.30 & 86.31 & 86.31 & 86.30 & 86.12 & 72.06 & 56.26 \\
\hline 7.00 & 58.59 & 83.56 & 86.38 & 86.44 & 86.45 & 86.45 & 86.44 & 86.25 & 72.22 & 56.45 \\
\hline 8.00 & 59.13 & 85.48 & 88.43 & 88.50 & 88.50 & 88.50 & 88.49 & 88.30 & 73.55 & 56.81 \\
\hline 9.00 & 59.38 & 85.63 & 88.57 & 88.64 & 88.65 & 88.65 & 88.63 & 88.44 & 73.88 & 57.81 \\
\hline 10.00 & 59.74 & 86.59 & 89.59 & 89.66 & 89.67 & 89.67 & 89.65 & 89.45 & 74.50 & 57.90 \\
\hline 11.00 & 59.98 & 86.73 & 89.73 & 89.80 & 89.81 & 89.81 & 89.79 & 89.59 & 74.59 & 57.72 \\
\hline 12.00 & 60.30 & 87.25 & 90.27 & 90.34 & 90.36 & 90.36 & 90.33 & 90.13 & 75.00 & 57.81 \\
\hline 13.00 & 60.52 & 87.39 & 90.41 & 90.48 & 90.50 & 90.50 & 90.47 & 90.26 & 75.18 & 58.07 \\
\hline 14.00 & 60.77 & 87.72 & 90.75 & 90.83 & 90.84 & 90.84 & 90.81 & 90.60 & 75.46 & 58.34 \\
\hline 15.00 & 60.98 & 87.86 & 90.88 & 90.97 & 90.98 & 90.98 & 90.95 & 90.74 & 75.60 & 58.46 \\
\hline 16.00 & 61.22 & 88.06 & 91.09 & 91.17 & 91.19 & 91.19 & 91.15 & 90.94 & 75.80 & 58.57 \\
\hline 17.00 & 61.42 & 88.20 & 91.22 & 91.31 & 91.33 & 91.32 & 91.29 & 91.07 & 76.06 & 59.45 \\
\hline 18.00 & 61.61 & 88.34 & 91.36 & 91.44 & 91.46 & 91.46 & 91.42 & 91.21 & 76.32 & 59.89 \\
\hline 19.00 & 61.79 & 88.48 & 91.49 & 91.58 & 91.60 & 91.60 & 91.56 & 91.34 & 76.37 & 59.89 \\
\hline 20.00 & 61.97 & 88.49 & 91.49 & 91.58 & 91.60 & 91.60 & 91.56 & 91.34 & 76.35 & 59.53 \\
\hline 21.00 & 62.12 & 88.61 & 91.60 & 91.70 & 91.72 & 91.71 & 91.67 & 91.45 & 76.47 & 59.46 \\
\hline 22.00 & 58.70 & 59.73 & 60.54 & 60.62 & 60.65 & 60.64 & 60.58 & 60.32 & 58.40 & 57.53 \\
\hline 22.50 & 57.65 & 55.96 & 55.96 & 56.02 & 56.04 & 56.04 & 55.98 & 55.89 & 56.13 & 57.64 \\
\hline
\end{tabular}


Table 31. Temperature $\left({ }^{\circ} \mathrm{C}\right)$ map of the west side of plate 1T2 at EOC 143A (100.9 EFPD).

\begin{tabular}{|c||c|c|c|c|c|c|c|c|c|c|}
\hline \multirow{2}{*}{$\begin{array}{c}\text { Length of } \\
\text { Plate (in) }\end{array}$} & \multicolumn{10}{|c|}{ Width of Plate (inches) } \\
\hline 0.125 & 52.33 & 51.79 & 51.77 & 51.77 & 51.77 & 51.77 & 51.77 & 51.77 & 51.85 & 52.31 \\
\hline 1.00 & 53.65 & 52.38 & 52.48 & 52.49 & 52.49 & 52.49 & 52.49 & 52.44 & 52.37 & 53.40 \\
\hline 2.00 & 56.82 & 76.26 & 78.55 & 78.60 & 78.60 & 78.60 & 78.60 & 78.46 & 67.12 & 54.87 \\
\hline 3.00 & 57.24 & 76.48 & 78.78 & 78.83 & 78.83 & 78.83 & 78.83 & 78.67 & 67.35 & 54.93 \\
\hline 4.00 & 58.08 & 80.72 & 83.35 & 83.41 & 83.41 & 83.41 & 83.40 & 83.23 & 70.17 & 55.56 \\
\hline 5.00 & 58.40 & 80.88 & 83.51 & 83.56 & 83.57 & 83.57 & 83.56 & 83.38 & 70.33 & 55.68 \\
\hline 6.00 & 59.03 & 83.45 & 86.27 & 86.33 & 86.34 & 86.34 & 86.32 & 86.14 & 72.07 & 56.06 \\
\hline 7.00 & 59.34 & 83.60 & 86.42 & 86.48 & 86.49 & 86.49 & 86.47 & 86.28 & 72.23 & 56.29 \\
\hline 8.00 & 59.92 & 85.53 & 88.47 & 88.54 & 88.55 & 88.55 & 88.53 & 88.34 & 73.57 & 56.64 \\
\hline 9.00 & 60.21 & 85.68 & 88.62 & 88.69 & 88.70 & 88.70 & 88.68 & 88.48 & 73.89 & 58.47 \\
\hline 10.00 & 60.61 & 86.65 & 89.65 & 89.73 & 89.74 & 89.74 & 89.71 & 89.51 & 74.53 & 58.36 \\
\hline 11.00 & 60.88 & 86.80 & 89.80 & 89.88 & 89.89 & 89.89 & 89.86 & 89.65 & 74.62 & 57.74 \\
\hline 12.00 & 61.24 & 87.32 & 90.35 & 90.43 & 90.44 & 90.44 & 90.41 & 90.20 & 75.04 & 57.53 \\
\hline 13.00 & 61.49 & 87.48 & 90.50 & 90.58 & 90.59 & 90.59 & 90.56 & 90.34 & 75.23 & 57.75 \\
\hline 14.00 & 61.77 & 87.82 & 90.85 & 90.93 & 90.95 & 90.95 & 90.91 & 90.69 & 75.52 & 58.13 \\
\hline 15.00 & 62.01 & 87.97 & 91.00 & 91.08 & 91.10 & 91.10 & 91.06 & 90.83 & 75.67 & 58.30 \\
\hline 16.00 & 62.28 & 88.19 & 91.21 & 91.30 & 91.32 & 91.32 & 91.28 & 91.05 & 75.87 & 58.40 \\
\hline 17.00 & 62.50 & 88.34 & 91.36 & 91.45 & 91.47 & 91.47 & 91.42 & 91.19 & 76.14 & 60.02 \\
\hline 18.00 & 62.71 & 88.48 & 91.50 & 91.60 & 91.62 & 91.61 & 91.57 & 91.34 & 76.40 & 60.72 \\
\hline 19.00 & 62.91 & 88.63 & 91.65 & 91.75 & 91.77 & 91.76 & 91.71 & 91.48 & 76.47 & 60.60 \\
\hline 20.00 & 63.10 & 88.66 & 91.66 & 91.76 & 91.78 & 91.78 & 91.73 & 91.49 & 76.46 & 59.60 \\
\hline 21.00 & 63.25 & 88.79 & 91.79 & 91.89 & 91.91 & 91.91 & 91.85 & 91.61 & 76.59 & 59.21 \\
\hline 22.00 & 59.88 & 59.88 & 60.69 & 60.78 & 60.81 & 60.81 & 60.73 & 60.46 & 58.52 & 57.46 \\
\hline 22.50 & 58.89 & 56.12 & 56.13 & 56.19 & 56.22 & 56.21 & 56.14 & 56.03 & 56.24 & 58.80 \\
\hline
\end{tabular}


Table 32. Temperature $\left({ }^{\circ} \mathrm{C}\right)$ map of the east side of plate 1B5 at EOC 143A (100.9 EFPD).

\begin{tabular}{|c||c|c|c|c|c|c|c|c|c|c|}
\hline \multirow{2}{*}{$\begin{array}{l}\text { Length of } \\
\text { Plate (in) }\end{array}$} & 0.05 & 0.25 & 0.50 & 0.75 & 1.00 & 1.25 & 1.50 & 1.75 & 2.00 & 2.24 \\
\hline 22.625 & 57.69 & 56.00 & 56.00 & 56.06 & 56.09 & 56.08 & 56.02 & 55.93 & 56.13 & 57.47 \\
\hline 23.00 & 58.78 & 59.72 & 60.50 & 60.58 & 60.61 & 60.60 & 60.54 & 60.29 & 58.42 & 57.62 \\
\hline 24.00 & 62.40 & 88.45 & 91.39 & 91.48 & 91.51 & 91.50 & 91.46 & 91.24 & 76.48 & 59.76 \\
\hline 25.00 & 62.57 & 88.68 & 91.63 & 91.72 & 91.75 & 91.74 & 91.69 & 91.48 & 76.71 & 60.11 \\
\hline 26.00 & 62.75 & 89.19 & 92.17 & 92.27 & 92.29 & 92.29 & 92.24 & 92.02 & 77.21 & 60.82 \\
\hline 27.00 & 62.86 & 89.33 & 92.31 & 92.41 & 92.44 & 92.43 & 92.38 & 92.16 & 77.46 & 61.13 \\
\hline 28.00 & 62.99 & 89.62 & 92.61 & 92.71 & 92.73 & 92.73 & 92.67 & 92.45 & 77.59 & 60.96 \\
\hline 29.00 & 63.09 & 89.76 & 92.75 & 92.86 & 92.88 & 92.88 & 92.82 & 92.59 & 77.64 & 60.60 \\
\hline 30.00 & 63.16 & 89.75 & 92.74 & 92.84 & 92.86 & 92.86 & 92.80 & 92.57 & 77.68 & 60.51 \\
\hline 31.00 & 63.23 & 89.88 & 92.86 & 92.97 & 92.99 & 92.99 & 92.93 & 92.70 & 77.83 & 60.65 \\
\hline 32.00 & 63.28 & 89.87 & 92.84 & 92.94 & 92.97 & 92.97 & 92.91 & 92.68 & 77.86 & 60.63 \\
\hline 33.00 & 63.34 & 89.99 & 92.97 & 93.07 & 93.10 & 93.09 & 93.03 & 92.80 & 77.96 & 60.62 \\
\hline 34.00 & 63.33 & 89.73 & 92.68 & 92.79 & 92.82 & 92.81 & 92.75 & 92.51 & 77.84 & 60.83 \\
\hline 35.00 & 63.36 & 89.85 & 92.80 & 92.91 & 92.94 & 92.94 & 92.87 & 92.64 & 78.04 & 61.32 \\
\hline 36.00 & 63.33 & 89.57 & 92.49 & 92.60 & 92.63 & 92.62 & 92.56 & 92.32 & 77.99 & 61.60 \\
\hline 37.00 & 63.35 & 89.68 & 92.61 & 92.72 & 92.75 & 92.74 & 92.68 & 92.44 & 78.00 & 61.35 \\
\hline 38.00 & 63.12 & 88.27 & 91.08 & 91.20 & 91.23 & 91.22 & 91.15 & 90.91 & 77.02 & 60.76 \\
\hline 39.00 & 63.11 & 88.37 & 91.19 & 91.30 & 91.33 & 91.33 & 91.26 & 91.02 & 77.11 & 60.59 \\
\hline 40.00 & 62.84 & 86.68 & 89.36 & 89.47 & 89.50 & 89.50 & 89.43 & 89.19 & 76.04 & 60.48 \\
\hline 41.00 & 62.80 & 86.77 & 89.45 & 89.57 & 89.60 & 89.59 & 89.52 & 89.28 & 76.13 & 60.52 \\
\hline 42.00 & 62.40 & 84.44 & 86.94 & 87.06 & 87.09 & 87.08 & 87.01 & 86.77 & 74.61 & 60.12 \\
\hline 43.00 & 62.33 & 84.51 & 87.02 & 87.13 & 87.16 & 87.16 & 87.08 & 86.84 & 74.68 & 60.22 \\
\hline 44.00 & 59.12 & 59.67 & 60.01 & 60.12 & 60.16 & 60.15 & 60.05 & 59.83 & 59.29 & 58.57 \\
\hline 45.00 & 58.55 & 59.14 & 59.31 & 59.40 & 59.44 & 59.43 & 59.33 & 59.18 & 58.94 & 58.29 \\
\hline
\end{tabular}


Table 33. Temperature $\left({ }^{\circ} \mathrm{C}\right)$ map of the west side of plate 1B5 at EOC 143A (100.9 EFPD).

\begin{tabular}{|c|c|c|c|c|c|c|c|c|c|c|}
\hline \multirow{2}{*}{$\begin{array}{c}\text { Length of } \\
\text { Plate (in) }\end{array}$} & \multicolumn{10}{|c|}{ Width of Plate (inches) } \\
\hline & 0.05 & 0.25 & 0.50 & 0.75 & 1.00 & 1.25 & 1.50 & 1.75 & 2.00 & 2.24 \\
\hline 22.625 & 58.85 & 56.16 & 56.17 & 56.23 & 56.26 & 56.26 & 56.19 & 56.08 & 56.24 & 58.27 \\
\hline 23.00 & 59.97 & 59.88 & 60.67 & 60.76 & 60.78 & 60.78 & 60.70 & 60.44 & 58.55 & 57.55 \\
\hline 24.00 & 63.54 & 88.67 & 91.61 & 91.72 & 91.74 & 91.74 & 91.68 & 91.44 & 76.63 & 59.52 \\
\hline 25.00 & 63.73 & 88.91 & 91.87 & 91.97 & 91.99 & 91.99 & 91.93 & 91.69 & 76.87 & 60.19 \\
\hline 26.00 & 63.89 & 89.44 & 92.42 & 92.53 & 92.55 & 92.55 & 92.48 & 92.24 & 77.37 & 61.55 \\
\hline 27.00 & 64.00 & 89.59 & 92.58 & 92.68 & 92.71 & 92.70 & 92.64 & 92.39 & 77.62 & 61.98 \\
\hline 28.00 & 64.12 & 89.89 & 92.88 & 92.99 & 93.02 & 93.01 & 92.94 & 92.69 & 77.77 & 61.56 \\
\hline 29.00 & 64.21 & 90.05 & 93.04 & 93.15 & 93.18 & 93.18 & 93.10 & 92.85 & 77.84 & 60.65 \\
\hline 30.00 & 64.26 & 90.05 & 93.04 & 93.15 & 93.18 & 93.17 & 93.10 & 92.84 & 77.88 & 60.30 \\
\hline 31.00 & 64.32 & 90.19 & 93.18 & 93.29 & 93.32 & 93.31 & 93.24 & 92.98 & 78.04 & 60.38 \\
\hline 32.00 & 64.34 & 90.18 & 93.17 & 93.28 & 93.31 & 93.30 & 93.22 & 92.97 & 78.08 & 60.24 \\
\hline 33.00 & 64.38 & 90.32 & 93.30 & 93.42 & 93.45 & 93.44 & 93.36 & 93.10 & 78.19 & 60.26 \\
\hline 34.00 & 64.34 & 90.07 & 93.03 & 93.15 & 93.18 & 93.17 & 93.09 & 92.83 & 78.08 & 60.81 \\
\hline 35.00 & 64.35 & 90.20 & 93.16 & 93.28 & 93.31 & 93.31 & 93.22 & 92.96 & 78.29 & 61.83 \\
\hline 36.00 & 64.29 & 89.93 & 92.86 & 92.98 & 93.01 & 93.00 & 92.92 & 92.65 & 78.24 & 62.37 \\
\hline 37.00 & 64.27 & 90.05 & 92.99 & 93.11 & 93.14 & 93.13 & 93.04 & 92.78 & 78.26 & 61.84 \\
\hline 38.00 & 64.02 & 88.65 & 91.48 & 91.60 & 91.63 & 91.62 & 91.53 & 91.26 & 77.29 & 60.70 \\
\hline 39.00 & 63.97 & 88.76 & 91.59 & 91.72 & 91.75 & 91.74 & 91.65 & 91.37 & 77.39 & 60.26 \\
\hline 40.00 & 63.66 & 87.08 & 89.78 & 89.90 & 89.93 & 89.92 & 89.83 & 89.56 & 76.33 & 60.12 \\
\hline 41.00 & 63.58 & 87.17 & 89.88 & 90.00 & 90.04 & 90.02 & 89.93 & 89.65 & 76.42 & 60.24 \\
\hline 42.00 & 63.14 & 84.86 & 87.38 & 87.50 & 87.54 & 87.53 & 87.43 & 87.16 & 74.91 & 59.77 \\
\hline 43.00 & 63.01 & 84.94 & 87.46 & 87.58 & 87.62 & 87.61 & 87.51 & 87.23 & 74.98 & 60.11 \\
\hline 44.00 & 59.80 & 60.01 & 60.37 & 60.48 & 60.52 & 60.51 & 60.39 & 60.14 & 59.56 & 58.92 \\
\hline 45.00 & 59.19 & 59.48 & 59.66 & 59.76 & 59.81 & 59.79 & 59.67 & 59.50 & 59.21 & 58.71 \\
\hline
\end{tabular}


Table 34. Temperature $\left({ }^{\circ} \mathrm{C}\right)$ map of the east side of plate $1 \mathrm{~T} 2$ at EOC $143 \mathrm{~B}$ (158.2 EFPD).

\begin{tabular}{|c||c|c|c|c|c|c|c|c|c|c|}
\hline \multirow{2}{*}{$\begin{array}{c}\text { Length of } \\
\text { Plate (in) }\end{array}$} & \multicolumn{10}{|c|}{ Width of Plate (inches) } \\
\hline & 0.05 & 0.25 & 0.50 & 0.75 & 1.00 & 1.25 & 1.50 & 1.75 & 2.00 & 2.24 \\
\hline 0.125 & 52.09 & 51.80 & 51.77 & 51.77 & 51.77 & 51.77 & 51.77 & 51.77 & 51.85 & 52.13 \\
\hline 1.00 & 53.41 & 52.32 & 52.38 & 52.39 & 52.39 & 52.39 & 52.39 & 52.35 & 52.36 & 53.28 \\
\hline 2.00 & 57.25 & 72.64 & 74.58 & 74.62 & 74.63 & 74.62 & 74.62 & 74.49 & 64.96 & 55.72 \\
\hline 3.00 & 57.68 & 72.89 & 74.83 & 74.88 & 74.88 & 74.88 & 74.87 & 74.74 & 65.23 & 55.99 \\
\hline 4.00 & 58.56 & 75.93 & 78.10 & 78.16 & 78.16 & 78.16 & 78.15 & 78.00 & 67.29 & 56.71 \\
\hline 5.00 & 58.89 & 76.14 & 78.32 & 78.37 & 78.38 & 78.38 & 78.37 & 78.21 & 67.51 & 56.94 \\
\hline 6.00 & 59.52 & 77.57 & 79.84 & 79.90 & 79.91 & 79.91 & 79.89 & 79.72 & 68.52 & 57.38 \\
\hline 7.00 & 59.88 & 77.82 & 80.09 & 80.16 & 80.17 & 80.17 & 80.15 & 79.97 & 68.79 & 57.64 \\
\hline 8.00 & 60.52 & 79.43 & 81.81 & 81.88 & 81.90 & 81.89 & 81.86 & 81.68 & 69.93 & 58.11 \\
\hline 9.00 & 60.84 & 79.66 & 82.04 & 82.12 & 82.14 & 82.14 & 82.10 & 81.91 & 70.35 & 59.18 \\
\hline 10.00 & 61.22 & 79.73 & 82.09 & 82.18 & 82.20 & 82.19 & 82.15 & 81.96 & 70.43 & 59.31 \\
\hline 11.00 & 61.52 & 79.97 & 82.33 & 82.42 & 82.44 & 82.44 & 82.40 & 82.20 & 70.60 & 59.18 \\
\hline 12.00 & 62.02 & 80.85 & 83.26 & 83.35 & 83.38 & 83.37 & 83.32 & 83.12 & 71.28 & 59.40 \\
\hline 13.00 & 62.31 & 81.10 & 83.51 & 83.61 & 83.63 & 83.63 & 83.57 & 83.36 & 71.56 & 59.72 \\
\hline 14.00 & 62.63 & 81.15 & 83.53 & 83.63 & 83.66 & 83.66 & 83.60 & 83.38 & 71.70 & 60.06 \\
\hline 15.00 & 62.91 & 81.40 & 83.78 & 83.89 & 83.92 & 83.92 & 83.85 & 83.63 & 71.94 & 60.24 \\
\hline 16.00 & 63.20 & 81.51 & 83.87 & 83.98 & 84.02 & 84.02 & 83.94 & 83.72 & 72.11 & 60.38 \\
\hline 17.00 & 63.46 & 81.76 & 84.12 & 84.24 & 84.28 & 84.28 & 84.20 & 83.97 & 72.49 & 61.34 \\
\hline 18.00 & 63.67 & 81.69 & 84.02 & 84.14 & 84.18 & 84.18 & 84.10 & 83.87 & 72.63 & 61.79 \\
\hline 19.00 & 63.92 & 81.94 & 84.27 & 84.40 & 84.44 & 84.44 & 84.35 & 84.11 & 72.80 & 61.86 \\
\hline 20.00 & 64.22 & 82.22 & 84.56 & 84.69 & 84.73 & 84.73 & 84.64 & 84.40 & 72.99 & 61.58 \\
\hline 21.00 & 64.43 & 82.46 & 84.79 & 84.93 & 84.97 & 84.97 & 84.87 & 84.63 & 73.22 & 61.55 \\
\hline 22.00 & 60.55 & 60.63 & 61.23 & 61.36 & 61.41 & 61.40 & 61.29 & 61.04 & 59.68 & 59.07 \\
\hline 22.50 & 59.19 & 57.92 & 57.95 & 58.06 & 58.11 & 58.11 & 58.00 & 57.86 & 58.05 & 58.91 \\
\hline
\end{tabular}


Table 35. Temperature $\left({ }^{\circ} \mathrm{C}\right)$ map of the west side of plate 1T2 at EOC 143B (158.2 EFPD).

\begin{tabular}{|c||c|c|c|c|c|c|c|c|c|c|}
\hline \multirow{2}{*}{$\begin{array}{c}\text { Length of } \\
\text { Plate (in) }\end{array}$} & \multicolumn{10}{|c|}{ Width of Plate (inches) } \\
\hline & 0.05 & 0.25 & 0.50 & 0.75 & 1.00 & 1.25 & 1.50 & 1.75 & 2.00 & 2.24 \\
\hline 0.125 & 52.34 & 51.80 & 51.77 & 51.77 & 51.77 & 51.77 & 51.77 & 51.77 & 51.85 & 52.33 \\
\hline 1.00 & 53.74 & 52.32 & 52.38 & 52.39 & 52.39 & 52.39 & 52.39 & 52.34 & 52.36 & 53.52 \\
\hline 2.00 & 56.89 & 72.61 & 74.55 & 74.59 & 74.60 & 74.60 & 74.59 & 74.46 & 64.94 & 55.21 \\
\hline 3.00 & 57.34 & 72.82 & 74.76 & 74.80 & 74.81 & 74.81 & 74.80 & 74.66 & 65.18 & 55.31 \\
\hline 4.00 & 58.10 & 75.81 & 77.98 & 78.03 & 78.03 & 78.03 & 78.02 & 77.88 & 67.19 & 55.95 \\
\hline 5.00 & 58.45 & 75.96 & 78.13 & 78.18 & 78.19 & 78.19 & 78.18 & 78.02 & 67.36 & 56.09 \\
\hline 6.00 & 58.99 & 77.32 & 79.58 & 79.64 & 79.64 & 79.64 & 79.63 & 79.47 & 68.31 & 56.47 \\
\hline 7.00 & 59.36 & 77.49 & 79.75 & 79.81 & 79.82 & 79.81 & 79.80 & 79.63 & 68.51 & 56.74 \\
\hline 8.00 & 59.92 & 79.02 & 81.39 & 81.45 & 81.46 & 81.46 & 81.44 & 81.27 & 69.59 & 57.13 \\
\hline 9.00 & 60.24 & 79.18 & 81.54 & 81.60 & 81.61 & 81.61 & 81.59 & 81.42 & 69.93 & 59.03 \\
\hline 10.00 & 60.56 & 79.17 & 81.50 & 81.57 & 81.58 & 81.58 & 81.56 & 81.38 & 69.94 & 58.92 \\
\hline 11.00 & 60.86 & 79.32 & 81.66 & 81.72 & 81.74 & 81.73 & 81.71 & 81.53 & 70.04 & 58.32 \\
\hline 12.00 & 61.31 & 80.11 & 82.48 & 82.55 & 82.56 & 82.56 & 82.53 & 82.35 & 70.64 & 58.17 \\
\hline 13.00 & 61.60 & 80.27 & 82.64 & 82.71 & 82.72 & 82.72 & 82.69 & 82.50 & 70.84 & 58.43 \\
\hline 14.00 & 61.87 & 80.22 & 82.56 & 82.64 & 82.65 & 82.65 & 82.61 & 82.43 & 70.90 & 58.84 \\
\hline 15.00 & 62.14 & 80.38 & 82.72 & 82.79 & 82.81 & 82.81 & 82.77 & 82.58 & 71.06 & 59.04 \\
\hline 16.00 & 62.40 & 80.38 & 82.69 & 82.77 & 82.79 & 82.79 & 82.75 & 82.55 & 71.14 & 59.16 \\
\hline 17.00 & 62.65 & 80.54 & 82.85 & 82.93 & 82.95 & 82.94 & 82.90 & 82.71 & 71.43 & 60.86 \\
\hline 18.00 & 62.84 & 80.37 & 82.65 & 82.73 & 82.75 & 82.75 & 82.70 & 82.50 & 71.49 & 61.54 \\
\hline 19.00 & 63.06 & 80.52 & 82.80 & 82.88 & 82.90 & 82.90 & 82.85 & 82.65 & 71.58 & 61.48 \\
\hline 20.00 & 63.31 & 80.70 & 82.97 & 83.06 & 83.08 & 83.08 & 83.03 & 82.83 & 71.68 & 60.52 \\
\hline 21.00 & 63.49 & 80.85 & 83.11 & 83.20 & 83.22 & 83.22 & 83.16 & 82.96 & 71.83 & 60.14 \\
\hline 22.00 & 60.53 & 59.34 & 59.88 & 59.97 & 60.00 & 60.00 & 59.92 & 59.70 & 58.41 & 58.31 \\
\hline 22.50 & 59.57 & 56.58 & 56.56 & 56.62 & 56.66 & 56.65 & 56.57 & 56.48 & 56.72 & 59.61 \\
\hline
\end{tabular}


Table 36. Temperature $\left({ }^{\circ} \mathrm{C}\right)$ map of the east side of plate $1 \mathrm{~B} 5$ at EOC 143B (158.2 EFPD).

\begin{tabular}{|c||c|c|c|c|c|c|c|c|c|c|}
\hline \multirow{2}{*}{$\begin{array}{l}\text { Length of } \\
\text { Plate (in) }\end{array}$} & 0.05 & 0.25 & 0.50 & 0.75 & 1.00 & 1.25 & 1.50 & 1.75 & 2.00 & 2.24 \\
\hline 22.625 & 59.27 & 57.96 & 58.00 & 58.11 & 58.16 & 58.15 & 58.04 & 57.91 & 58.06 & 58.76 \\
\hline 23.00 & 60.47 & 60.70 & 61.29 & 61.42 & 61.47 & 61.46 & 61.35 & 61.10 & 59.76 & 59.03 \\
\hline 24.00 & 64.22 & 82.53 & 84.83 & 84.97 & 85.02 & 85.02 & 84.92 & 84.67 & 73.39 & 61.47 \\
\hline 25.00 & 64.44 & 82.81 & 85.12 & 85.26 & 85.31 & 85.31 & 85.21 & 84.96 & 73.67 & 61.86 \\
\hline 26.00 & 64.64 & 83.27 & 85.60 & 85.75 & 85.80 & 85.80 & 85.69 & 85.44 & 74.15 & 62.59 \\
\hline 27.00 & 64.81 & 83.49 & 85.82 & 85.97 & 86.02 & 86.02 & 85.91 & 85.65 & 74.46 & 62.91 \\
\hline 28.00 & 65.00 & 84.15 & 86.52 & 86.67 & 86.72 & 86.71 & 86.60 & 86.35 & 74.86 & 62.82 \\
\hline 29.00 & 65.15 & 84.38 & 86.75 & 86.90 & 86.96 & 86.95 & 86.84 & 86.58 & 74.99 & 62.50 \\
\hline 30.00 & 65.22 & 84.22 & 86.57 & 86.72 & 86.78 & 86.77 & 86.66 & 86.39 & 74.95 & 62.41 \\
\hline 31.00 & 65.33 & 84.42 & 86.77 & 86.93 & 86.99 & 86.98 & 86.86 & 86.60 & 75.17 & 62.58 \\
\hline 32.00 & 65.44 & 84.76 & 87.12 & 87.28 & 87.33 & 87.33 & 87.21 & 86.94 & 75.44 & 62.60 \\
\hline 33.00 & 65.55 & 84.98 & 87.35 & 87.51 & 87.57 & 87.56 & 87.44 & 87.17 & 75.63 & 62.65 \\
\hline 34.00 & 65.66 & 85.54 & 87.94 & 88.10 & 88.16 & 88.16 & 88.03 & 87.76 & 76.07 & 62.94 \\
\hline 35.00 & 65.74 & 85.74 & 88.14 & 88.31 & 88.36 & 88.36 & 88.23 & 87.96 & 76.34 & 63.45 \\
\hline 36.00 & 65.66 & 85.32 & 87.68 & 87.85 & 87.91 & 87.90 & 87.77 & 87.49 & 76.22 & 63.67 \\
\hline 37.00 & 65.72 & 85.51 & 87.87 & 88.04 & 88.10 & 88.10 & 87.96 & 87.68 & 76.30 & 63.47 \\
\hline 38.00 & 65.70 & 85.57 & 87.94 & 88.10 & 88.17 & 88.16 & 88.03 & 87.74 & 76.32 & 63.02 \\
\hline 39.00 & 65.73 & 85.75 & 88.12 & 88.29 & 88.35 & 88.35 & 88.21 & 87.93 & 76.47 & 62.88 \\
\hline 40.00 & 65.48 & 84.74 & 87.02 & 87.19 & 87.26 & 87.25 & 87.11 & 86.82 & 75.87 & 62.78 \\
\hline 41.00 & 65.48 & 84.90 & 87.19 & 87.36 & 87.42 & 87.42 & 87.28 & 86.99 & 76.03 & 62.84 \\
\hline 42.00 & 65.10 & 83.45 & 85.61 & 85.79 & 85.85 & 85.85 & 85.70 & 85.42 & 75.10 & 62.44 \\
\hline 43.00 & 65.05 & 83.59 & 85.75 & 85.93 & 85.99 & 85.99 & 85.84 & 85.55 & 75.22 & 62.56 \\
\hline 44.00 & 61.66 & 62.95 & 63.32 & 63.48 & 63.55 & 63.54 & 63.38 & 63.12 & 62.54 & 60.64 \\
\hline 45.00 & 61.00 & 62.54 & 62.78 & 62.93 & 63.00 & 62.99 & 62.83 & 62.63 & 62.25 & 60.25 \\
\hline
\end{tabular}


Table 37. Temperature $\left({ }^{\circ} \mathrm{C}\right)$ map of the west side of plate 1B5 at EOC 143B (158.2 EFPD).

\begin{tabular}{|c|c|c|c|c|c|c|c|c|c|c|}
\hline \multirow{2}{*}{$\begin{array}{c}\text { Length of } \\
\text { Plate (in) }\end{array}$} & \multicolumn{10}{|c|}{ Width of Plate (inches) } \\
\hline & 0.05 & 0.25 & 0.50 & 0.75 & 1.00 & 1.25 & 1.50 & 1.75 & 2.00 & 2.24 \\
\hline 22.625 & 59.55 & 56.62 & 56.59 & 56.66 & 56.70 & 56.69 & 56.61 & 56.52 & 56.73 & 59.09 \\
\hline 23.00 & 60.55 & 59.37 & 59.90 & 59.99 & 60.02 & 60.01 & 59.94 & 59.73 & 58.44 & 58.34 \\
\hline 24.00 & 63.59 & 80.74 & 82.97 & 83.06 & 83.08 & 83.07 & 83.02 & 82.82 & 71.85 & 60.25 \\
\hline 25.00 & 63.79 & 80.96 & 83.19 & 83.28 & 83.31 & 83.30 & 83.24 & 83.04 & 72.07 & 60.96 \\
\hline 26.00 & 63.95 & 81.37 & 83.62 & 83.71 & 83.73 & 83.73 & 83.67 & 83.47 & 72.50 & 62.32 \\
\hline 27.00 & 64.07 & 81.52 & 83.77 & 83.87 & 83.89 & 83.88 & 83.82 & 83.62 & 72.75 & 62.74 \\
\hline 28.00 & 64.24 & 82.12 & 84.41 & 84.50 & 84.53 & 84.52 & 84.46 & 84.25 & 73.10 & 62.39 \\
\hline 29.00 & 64.34 & 82.28 & 84.57 & 84.66 & 84.69 & 84.68 & 84.62 & 84.41 & 73.17 & 61.51 \\
\hline 30.00 & 64.36 & 82.06 & 84.32 & 84.41 & 84.44 & 84.43 & 84.36 & 84.15 & 73.07 & 61.15 \\
\hline 31.00 & 64.43 & 82.20 & 84.46 & 84.55 & 84.58 & 84.57 & 84.50 & 84.29 & 73.23 & 61.24 \\
\hline 32.00 & 64.50 & 82.46 & 84.74 & 84.83 & 84.86 & 84.85 & 84.78 & 84.57 & 73.45 & 61.14 \\
\hline 33.00 & 64.55 & 82.62 & 84.89 & 84.99 & 85.02 & 85.01 & 84.94 & 84.72 & 73.57 & 61.26 \\
\hline 34.00 & 64.63 & 83.12 & 85.43 & 85.52 & 85.55 & 85.54 & 85.47 & 85.25 & 73.95 & 61.82 \\
\hline 35.00 & 64.65 & 83.25 & 85.56 & 85.66 & 85.69 & 85.68 & 85.60 & 85.38 & 74.17 & 62.84 \\
\hline 36.00 & 64.54 & 82.76 & 85.03 & 85.13 & 85.15 & 85.14 & 85.07 & 84.85 & 73.99 & 63.30 \\
\hline 37.00 & 64.54 & 82.89 & 85.16 & 85.26 & 85.28 & 85.27 & 85.20 & 84.98 & 74.02 & 62.85 \\
\hline 38.00 & 64.49 & 82.88 & 85.15 & 85.25 & 85.28 & 85.26 & 85.19 & 84.96 & 73.97 & 61.84 \\
\hline 39.00 & 64.45 & 83.00 & 85.27 & 85.37 & 85.40 & 85.39 & 85.31 & 85.08 & 74.08 & 61.42 \\
\hline 40.00 & 64.21 & 81.91 & 84.09 & 84.19 & 84.22 & 84.21 & 84.13 & 83.90 & 73.42 & 61.30 \\
\hline 41.00 & 64.14 & 82.02 & 84.20 & 84.30 & 84.33 & 84.32 & 84.24 & 84.01 & 73.52 & 61.43 \\
\hline 42.00 & 63.77 & 80.48 & 82.54 & 82.64 & 82.67 & 82.65 & 82.57 & 82.34 & 72.52 & 61.00 \\
\hline 43.00 & 63.65 & 80.56 & 82.63 & 82.73 & 82.76 & 82.74 & 82.66 & 82.43 & 72.61 & 61.35 \\
\hline 44.00 & 60.73 & 60.60 & 60.89 & 61.00 & 61.04 & 61.02 & 60.91 & 60.70 & 60.21 & 60.16 \\
\hline 45.00 & 60.13 & 60.18 & 60.34 & 60.44 & 60.48 & 60.46 & 60.35 & 60.20 & 59.91 & 59.88 \\
\hline
\end{tabular}




\section{REFERENCES}

1. D.M. Wachs, “RERTR Fuel Development and Qualification Plan,” INL/EXT-05-01017, Revision 4, August 2009.

2. "Experiment Control Plan for the AFIP-1 Fuel Irradiation in the ATR," PLN-2630, April 2008.

3. M. A. Lillo, G. S. Chang. "As-Run Neutronic Analysis for the AFIP-1 Experiment Irradiated in the Center Flux Trap of the ATR, Cycles 142B, 143A, and 143B,” ECAR-1522. May 2011.

4. RERTR Project Personnel, "RERTR AFIP-1 Irradiation Experiment in the Advanced Test Reactor: As-Built Data Package,” 2770527, November 2008.

5. G. A. Moore, "Fuel Loading Calculations for AFIP-1 Irradiation Experiment,” ECAR-225, April 2008.

6. D.M. Wachs, "Hydraulic Testing of the AFIP Irradiation Vehicle for the ATR Center Flux Trap Position," ECAR-99. October 2007.

7. R. G. Ambrosek, "RELAP Analysis for AFIP-2 Flow Resistor,” ECAR-126, January 2008.

8. P. E. Murray, "Validation of ABAQUS Standard 6.7-3 Heat Transfer", ECAR-131, January 2008.

9. G. A. Roth, “AFIP-1 Thermal As-Run Analysis Results,” GAR-03-11, May 2011. 
Appendix A

\section{Individual Plate Power and Fission Density Plots}




\section{Appendix A \\ Power and Fission Density Plots}

A-1. Plate 1 T2

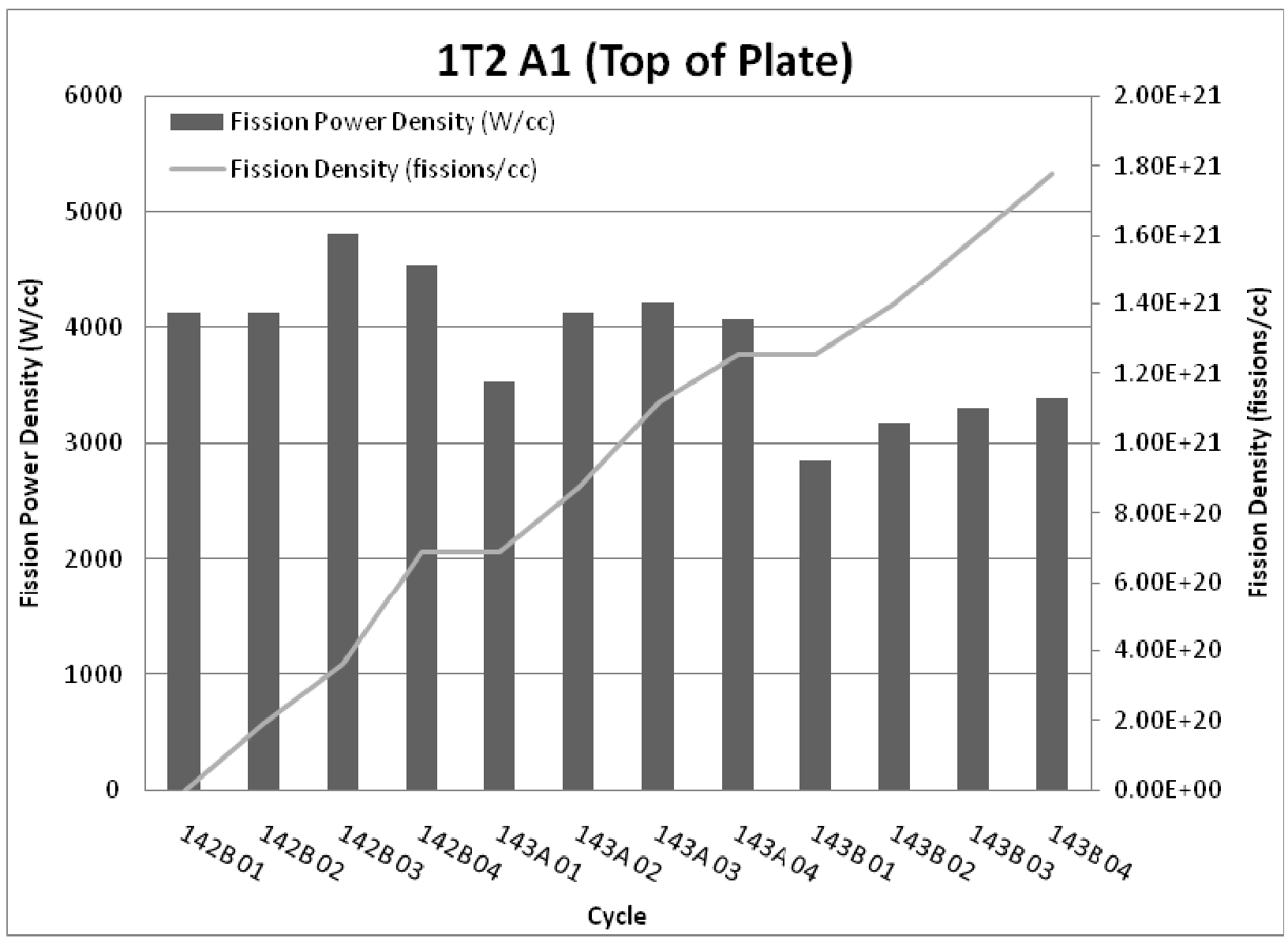



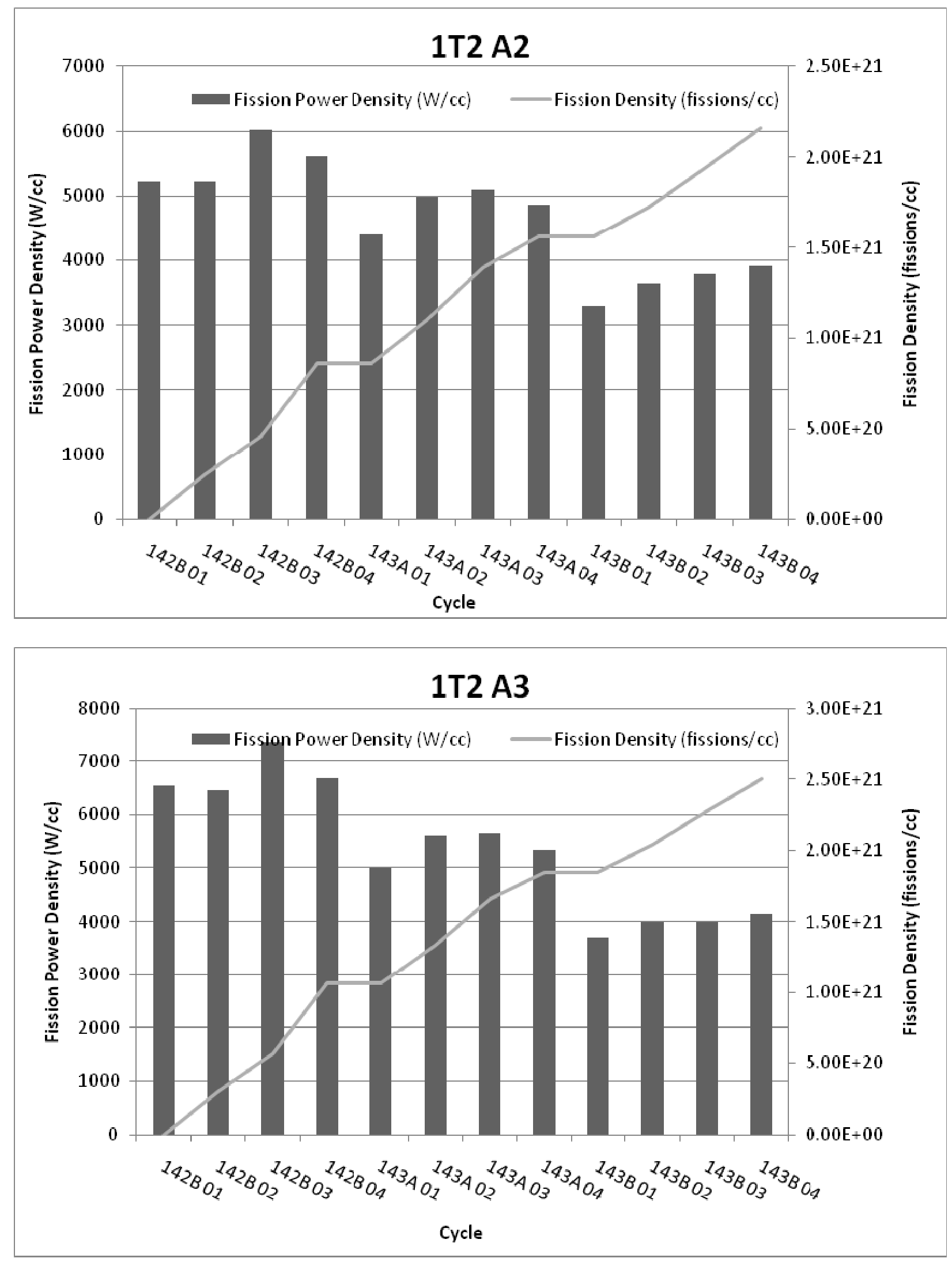

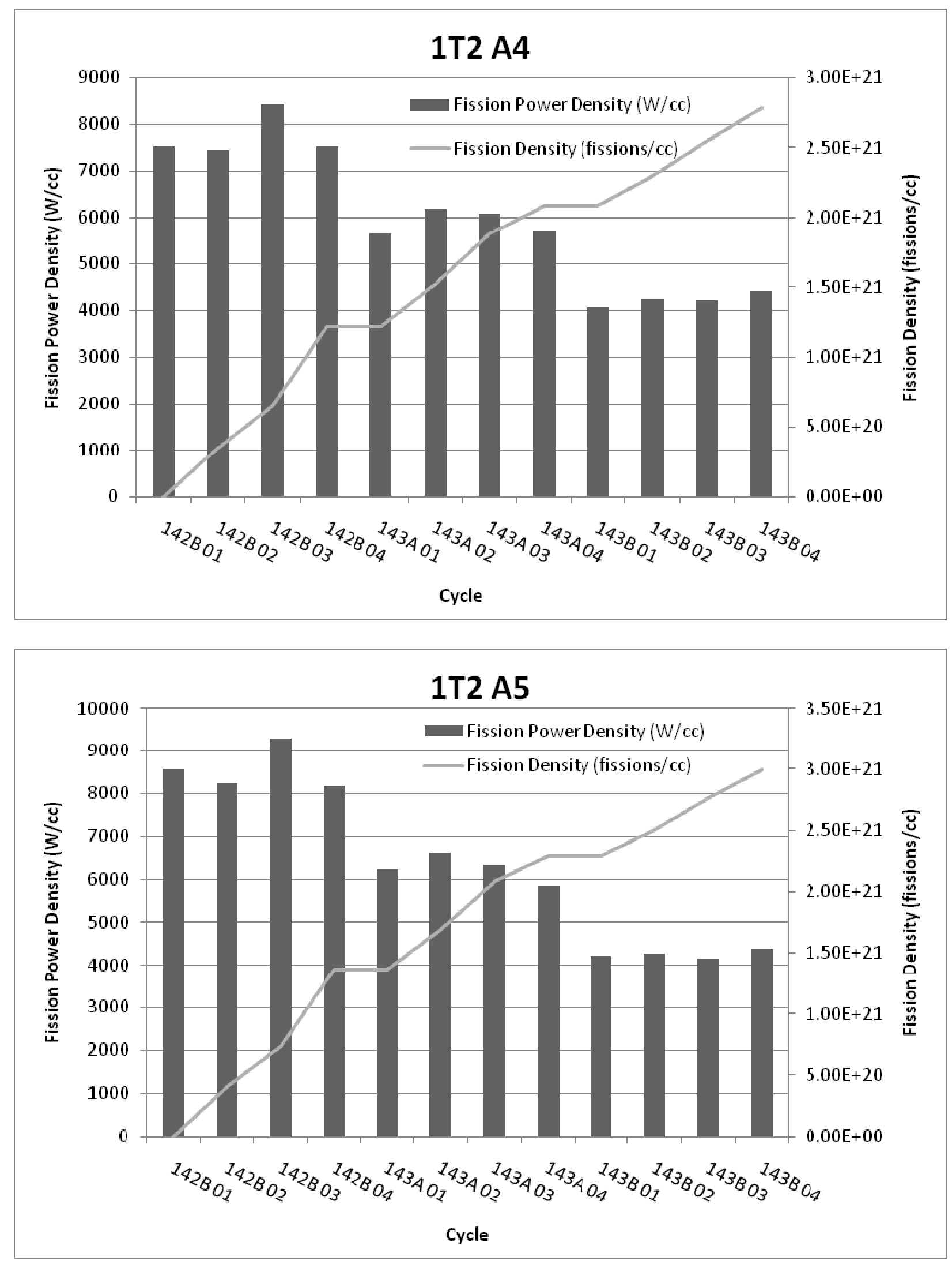

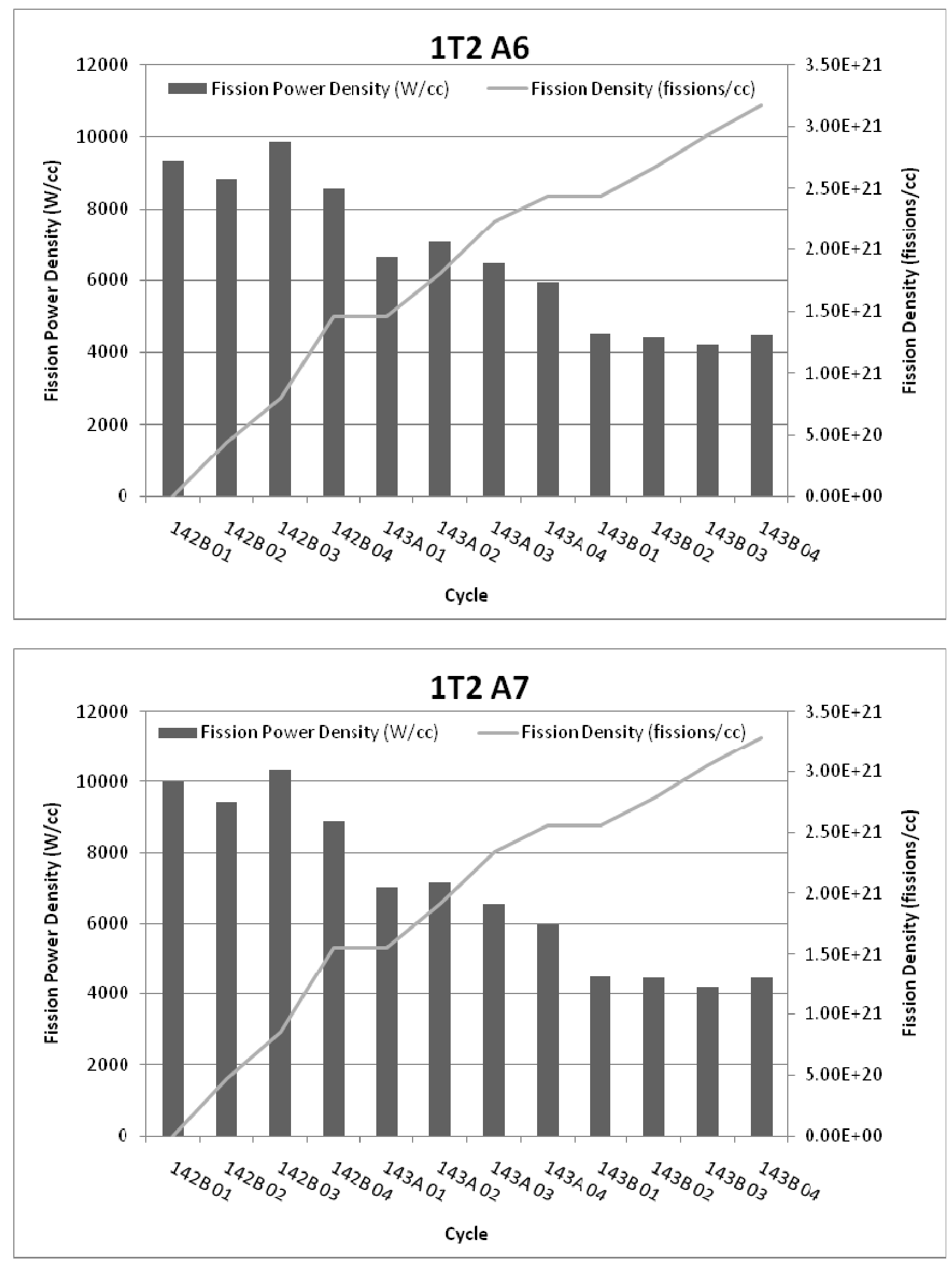

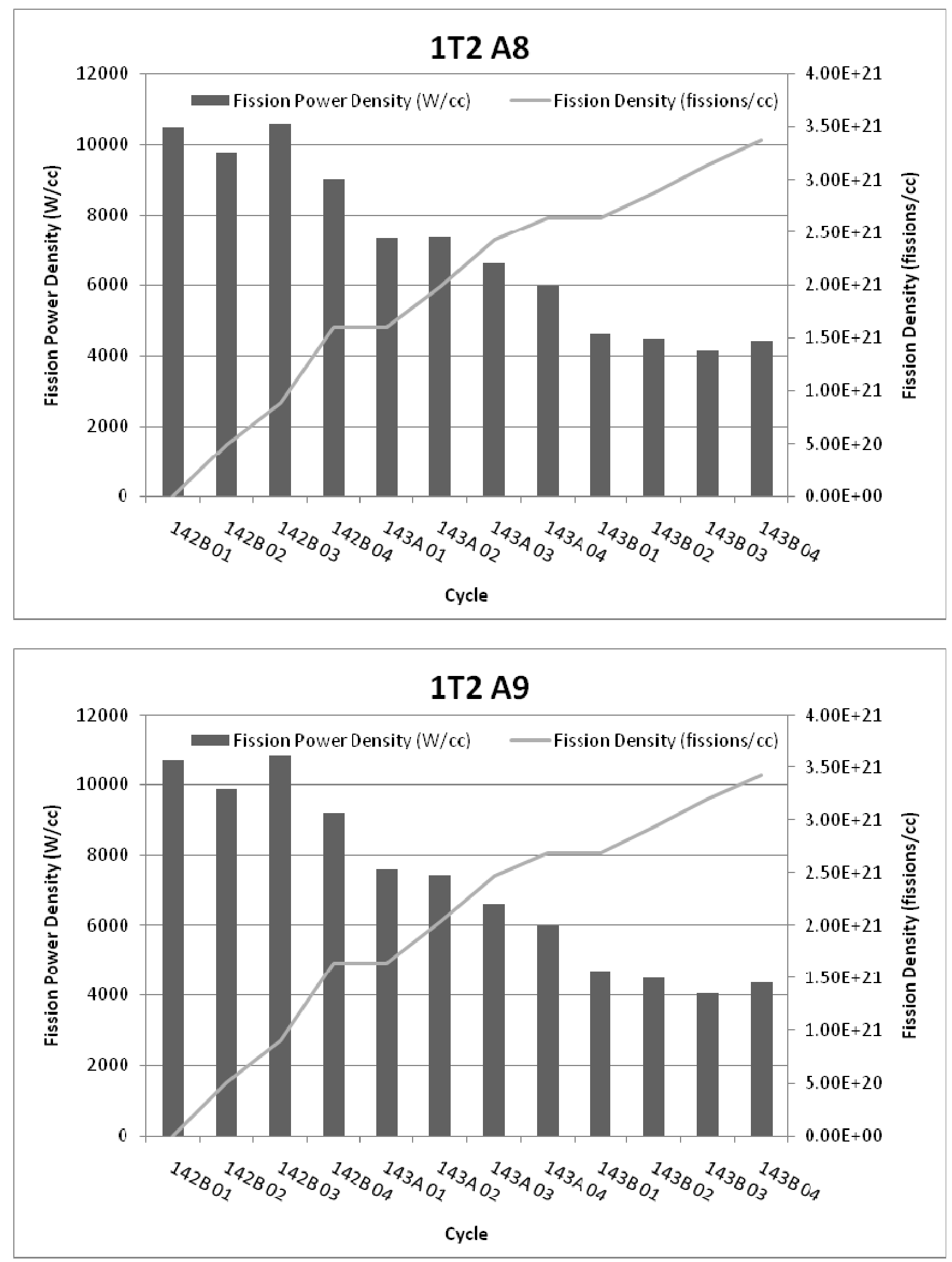


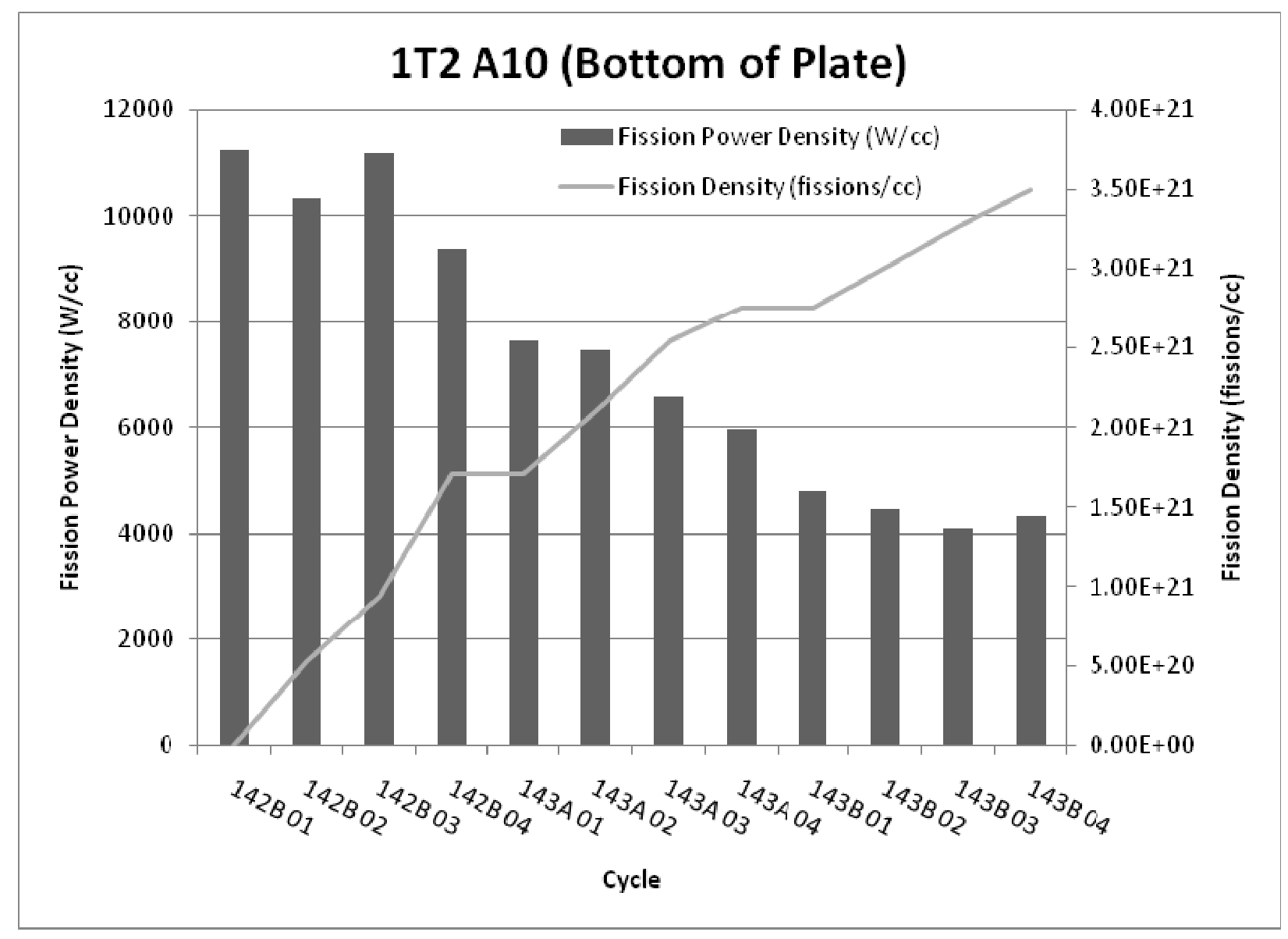




\section{A-2. Plate 1B5}
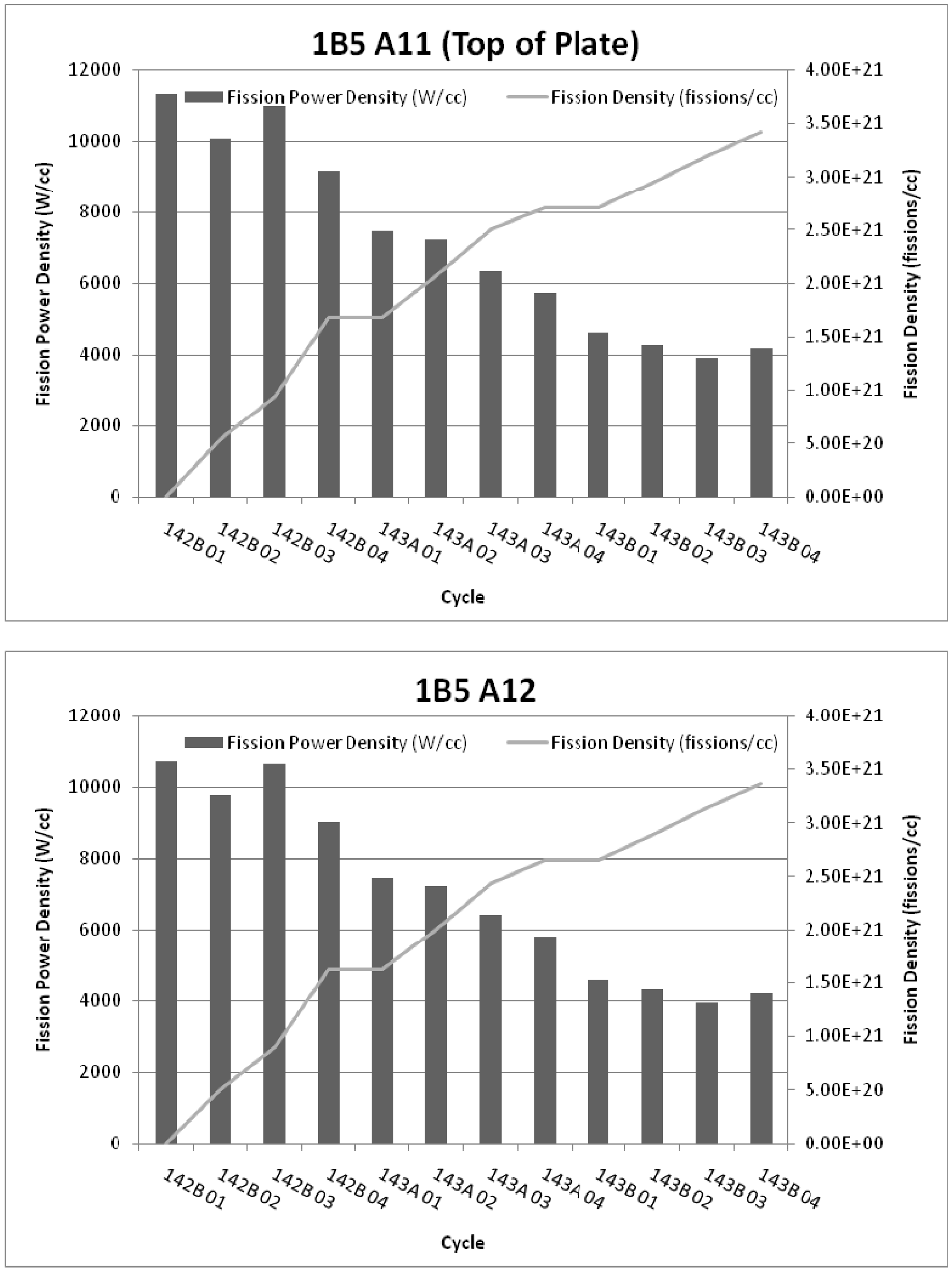

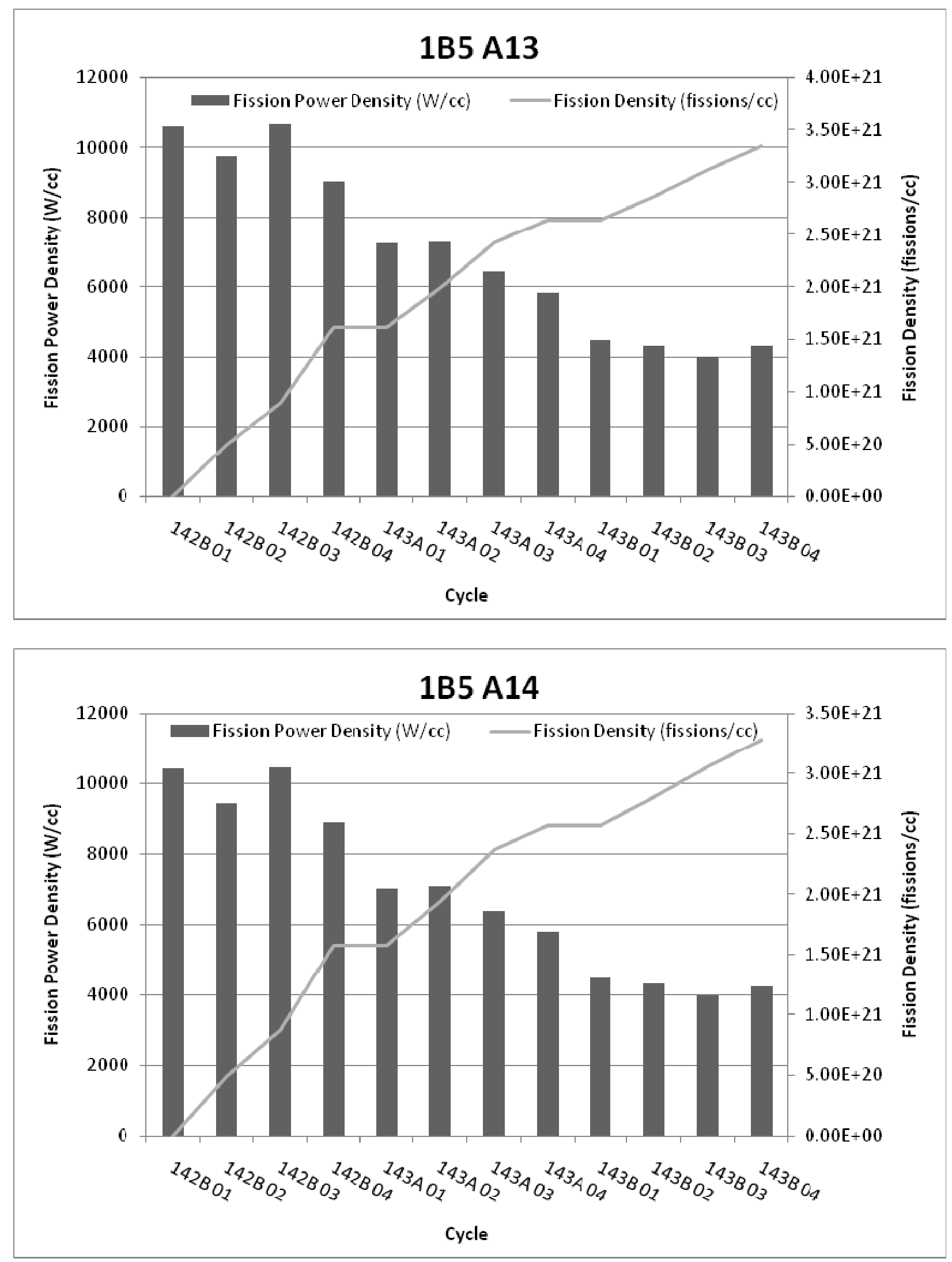

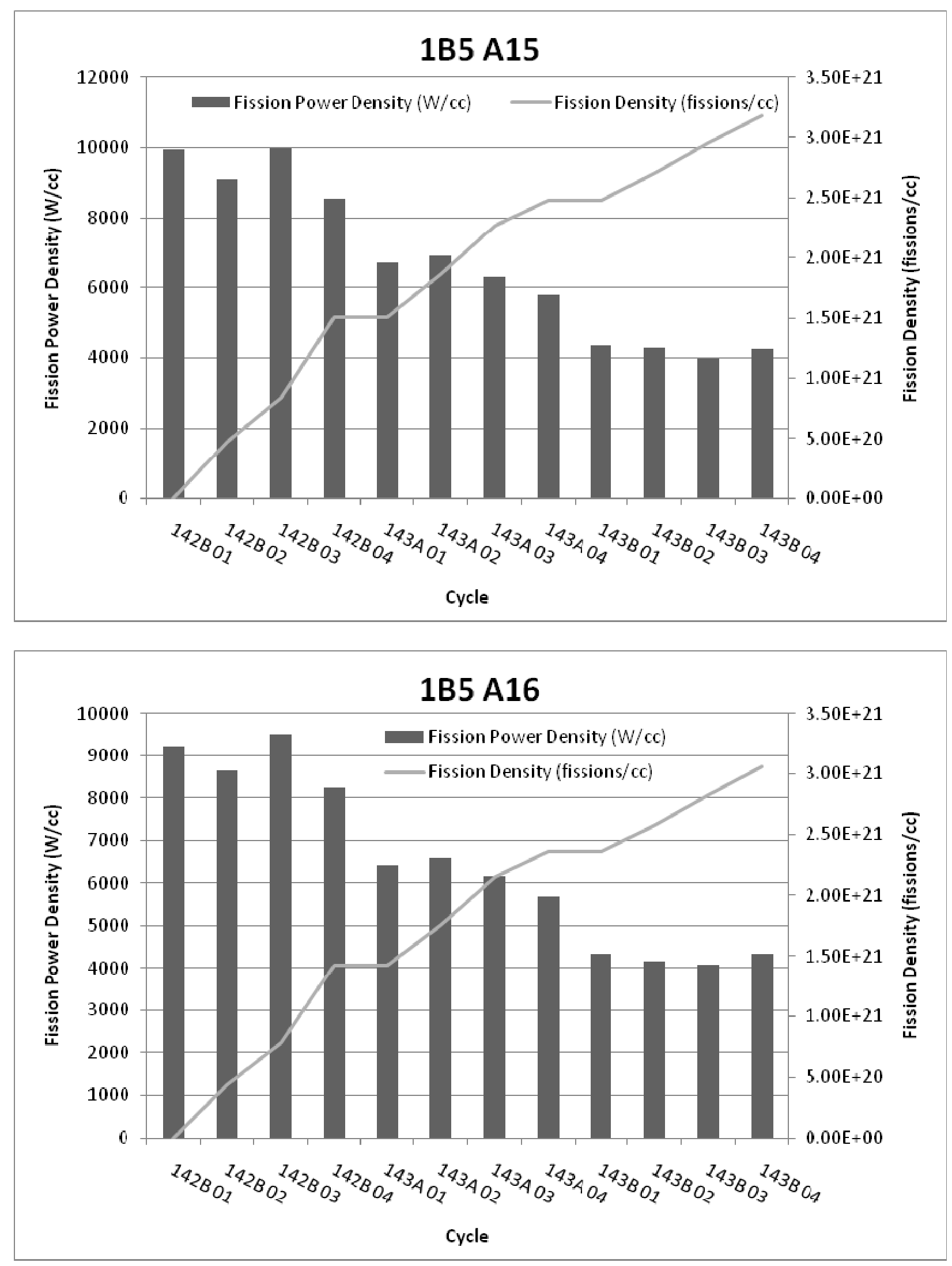

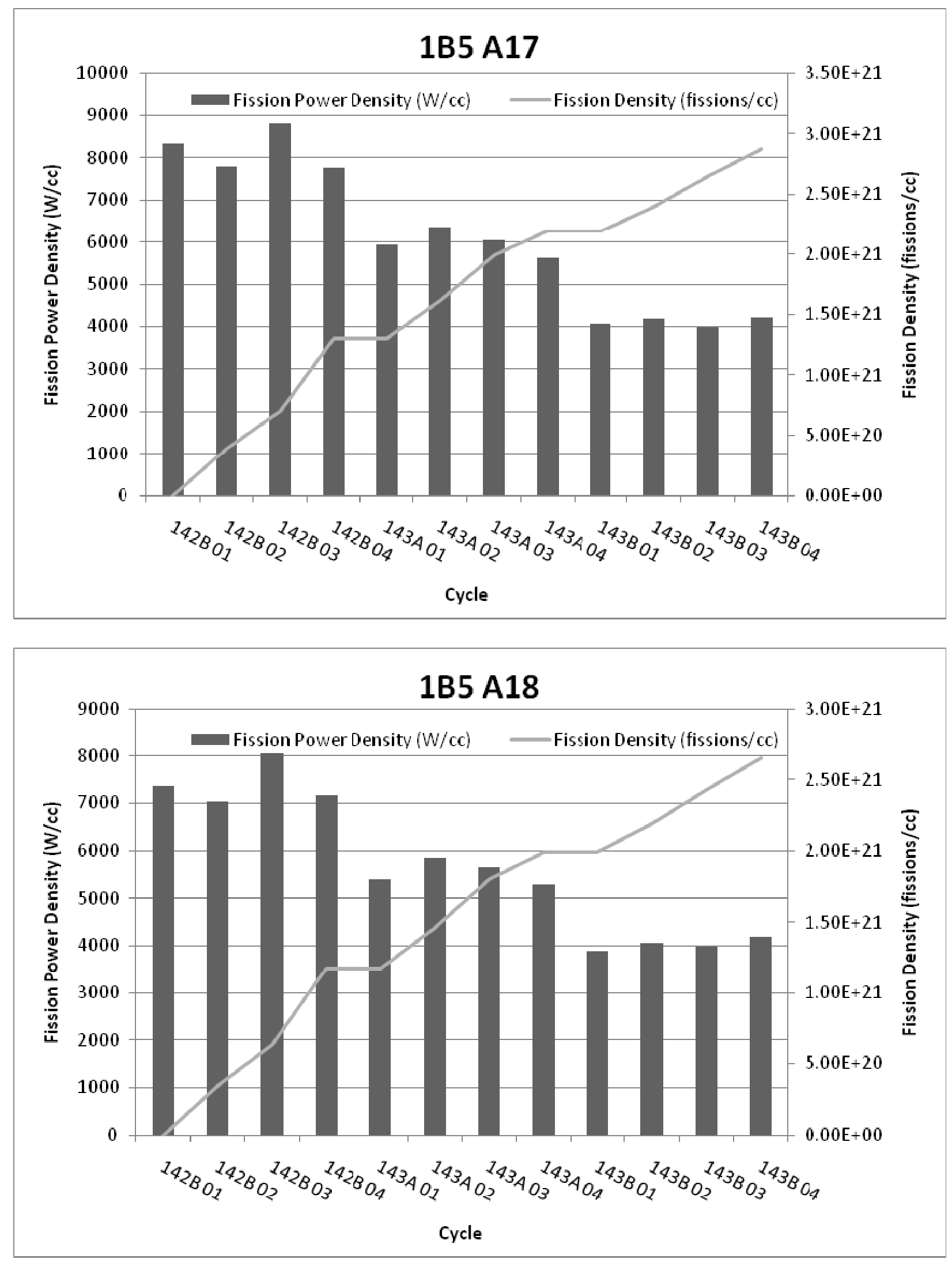

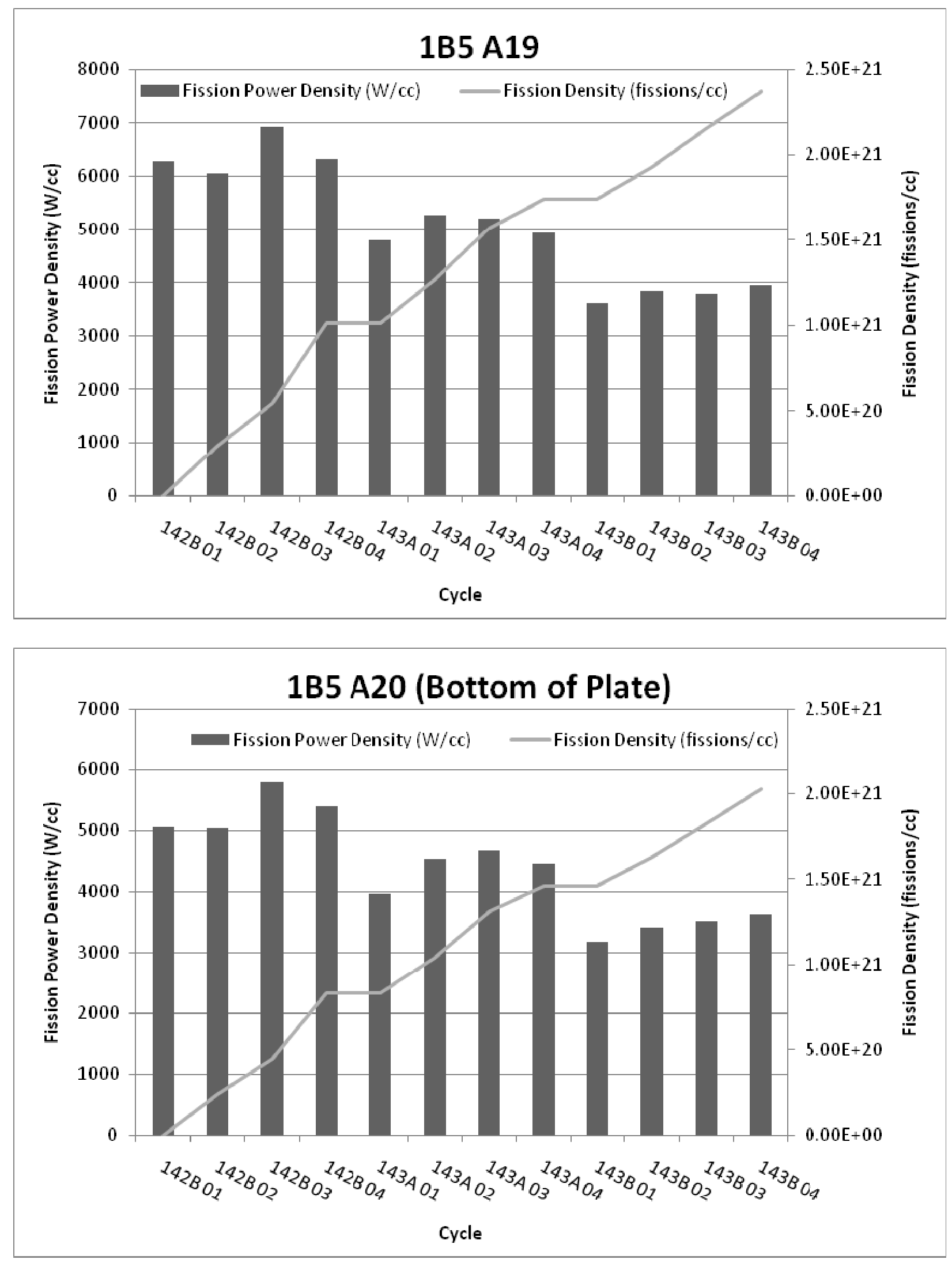
Appendix B

\section{Plate Surface Temperatures}




\section{Appendix B}

Table B - 1. Temperature $\left({ }^{\circ} \mathrm{C}\right)$ map of the east side of plate 1T2 at BOC 142B (0 EFPD).

\begin{tabular}{|c|c|c|c|c|c|c|c|c|c|c|}
\hline \multirow{2}{*}{$\begin{array}{l}\text { Length of } \\
\text { Plate (in) }\end{array}$} & \multicolumn{10}{|c|}{ Width of Plate (inches) } \\
\hline & 0.05 & 0.25 & 0.50 & 0.75 & 1.00 & 1.25 & 1.50 & 1.75 & 2.00 & 2.24 \\
\hline 0.125 & 52.10 & 51.80 & 51.77 & 51.77 & 51.77 & 51.77 & 51.77 & 51.78 & 51.86 & 52.14 \\
\hline 1.00 & 53.44 & 52.41 & 52.50 & 52.51 & 52.51 & 52.51 & 52.51 & 52.46 & 52.42 & 53.30 \\
\hline 2.00 & 57.50 & 76.58 & 78.86 & 78.91 & 78.91 & 78.91 & 78.90 & 78.76 & 67.46 & 55.81 \\
\hline 3.00 & 57.97 & 76.85 & 79.15 & 79.20 & 79.20 & 79.20 & 79.20 & 79.04 & 67.76 & 56.11 \\
\hline 4.00 & 59.23 & 82.74 & 85.48 & 85.54 & 85.54 & 85.54 & 85.53 & 85.36 & 71.69 & 57.06 \\
\hline 5.00 & 59.61 & 82.98 & 85.72 & 85.78 & 85.79 & 85.79 & 85.78 & 85.60 & 71.95 & 57.33 \\
\hline 6.00 & 61.02 & 89.65 & 92.86 & 92.94 & 92.95 & 92.94 & 92.93 & 92.73 & 76.44 & 58.23 \\
\hline 7.00 & 61.37 & 89.89 & 93.11 & 93.19 & 93.20 & 93.20 & 93.18 & 92.98 & 76.71 & 58.50 \\
\hline 8.00 & 62.56 & 94.79 & 98.33 & 98.42 & 98.44 & 98.44 & 98.41 & 98.19 & 80.12 & 59.29 \\
\hline 9.00 & 62.91 & 95.05 & 98.60 & 98.69 & 98.71 & 98.71 & 98.67 & 98.45 & 80.59 & 60.53 \\
\hline 10.00 & 64.09 & 100.15 & 104.32 & 104.43 & 104.46 & 104.46 & 104.41 & 104.14 & 84.00 & 61.11 \\
\hline 11.00 & 64.45 & 100.47 & 104.65 & 104.77 & 104.80 & 104.80 & 104.74 & 104.46 & 84.22 & 60.93 \\
\hline 12.00 & 65.46 & 104.41 & 108.87 & 109.01 & 109.04 & 109.03 & 108.98 & 108.67 & 86.85 & 61.45 \\
\hline 13.00 & 65.82 & 104.75 & 109.22 & 109.36 & 109.39 & 109.39 & 109.33 & 109.01 & 87.18 & 61.83 \\
\hline 14.00 & 66.67 & 108.15 & 112.85 & 113.00 & 113.03 & 113.03 & 112.96 & 112.63 & 89.45 & 62.52 \\
\hline 15.00 & 67.04 & 108.50 & 113.21 & 113.36 & 113.40 & 113.40 & 113.32 & 112.98 & 89.76 & 62.75 \\
\hline 16.00 & 67.77 & 111.10 & 115.98 & 116.15 & 116.19 & 116.18 & 116.10 & 115.75 & 91.52 & 63.15 \\
\hline 17.00 & 68.12 & 111.45 & 116.34 & 116.51 & 116.56 & 116.55 & 116.46 & 116.10 & 91.98 & 64.35 \\
\hline 18.00 & 68.62 & 112.89 & 117.86 & 118.04 & 118.08 & 118.08 & 117.99 & 117.61 & 93.12 & 65.12 \\
\hline 19.00 & 68.98 & 113.25 & 118.22 & 118.41 & 118.46 & 118.46 & 118.35 & 117.97 & 93.33 & 65.19 \\
\hline 20.00 & 69.71 & 116.05 & 121.21 & 121.40 & 121.45 & 121.45 & 121.34 & 120.95 & 95.17 & 65.01 \\
\hline 21.00 & 70.04 & 116.39 & 121.55 & 121.75 & 121.81 & 121.80 & 121.69 & 121.29 & 95.50 & 65.02 \\
\hline 22.00 & 62.78 & 66.48 & 68.03 & 68.22 & 68.28 & 68.28 & 68.13 & 67.64 & 63.97 & 60.63 \\
\hline 22.50 & 60.38 & 59.86 & 60.09 & 60.25 & 60.32 & 60.31 & 60.16 & 59.91 & 59.81 & 59.94 \\
\hline
\end{tabular}


Table B - 2.Temperature $\left({ }^{\circ} \mathrm{C}\right)$ map of the west side of plate 1T2 at BOC 142B (0 EFPD).

\begin{tabular}{|c||c|c|c|c|c|c|c|c|c|c|}
\hline \multirow{2}{*}{$\begin{array}{c}\text { Length of } \\
\text { Plate (in) }\end{array}$} & \multicolumn{10}{|c|}{ Width of Plate (inches) } \\
\hline & 0.05 & 0.25 & 0.50 & 0.75 & 1.00 & 1.25 & 1.50 & 1.75 & 2.00 & 2.24 \\
\hline 0.125 & 52.36 & 51.80 & 51.77 & 51.77 & 51.77 & 51.77 & 51.77 & 51.78 & 51.85 & 52.34 \\
\hline 1.00 & 53.81 & 52.40 & 52.50 & 52.51 & 52.51 & 52.51 & 52.51 & 52.45 & 52.42 & 53.57 \\
\hline 2.00 & 57.36 & 76.56 & 78.84 & 78.89 & 78.89 & 78.89 & 78.89 & 78.74 & 67.44 & 55.41 \\
\hline 3.00 & 57.87 & 76.80 & 79.10 & 79.15 & 79.15 & 79.15 & 79.15 & 78.99 & 67.72 & 55.53 \\
\hline 4.00 & 59.03 & 82.66 & 85.39 & 85.45 & 85.46 & 85.46 & 85.45 & 85.28 & 71.62 & 56.38 \\
\hline 5.00 & 59.44 & 82.86 & 85.59 & 85.65 & 85.66 & 85.66 & 85.65 & 85.47 & 71.84 & 56.56 \\
\hline 6.00 & 60.77 & 89.48 & 92.69 & 92.76 & 92.77 & 92.77 & 92.75 & 92.56 & 76.29 & 57.36 \\
\hline 7.00 & 61.20 & 89.71 & 92.92 & 92.99 & 93.00 & 93.00 & 92.98 & 92.78 & 76.54 & 57.67 \\
\hline 8.00 & 62.29 & 94.52 & 98.06 & 98.14 & 98.15 & 98.15 & 98.13 & 97.92 & 79.87 & 58.34 \\
\hline 9.00 & 62.67 & 94.73 & 98.26 & 98.35 & 98.36 & 98.36 & 98.33 & 98.12 & 80.28 & 60.57 \\
\hline 10.00 & 63.80 & 99.72 & 103.87 & 103.98 & 103.99 & 103.99 & 103.96 & 103.69 & 83.66 & 60.84 \\
\hline 11.00 & 64.18 & 99.97 & 104.13 & 104.24 & 104.26 & 104.26 & 104.22 & 103.94 & 83.82 & 60.09 \\
\hline 12.00 & 65.14 & 103.84 & 108.29 & 108.40 & 108.42 & 108.42 & 108.38 & 108.08 & 86.39 & 60.14 \\
\hline 13.00 & 65.50 & 104.11 & 108.56 & 108.68 & 108.71 & 108.70 & 108.65 & 108.35 & 86.67 & 60.46 \\
\hline 14.00 & 66.33 & 107.44 & 112.12 & 112.25 & 112.28 & 112.28 & 112.22 & 111.91 & 88.89 & 61.20 \\
\hline 15.00 & 66.69 & 107.72 & 112.41 & 112.54 & 112.57 & 112.57 & 112.51 & 112.18 & 89.14 & 61.48 \\
\hline 16.00 & 67.40 & 110.24 & 115.10 & 115.25 & 115.27 & 115.27 & 115.21 & 114.87 & 90.84 & 61.83 \\
\hline 17.00 & 67.75 & 110.52 & 115.39 & 115.54 & 115.57 & 115.56 & 115.49 & 115.14 & 91.23 & 63.98 \\
\hline 18.00 & 68.23 & 111.89 & 116.83 & 116.99 & 117.02 & 117.02 & 116.94 & 116.58 & 92.30 & 65.06 \\
\hline 19.00 & 68.57 & 112.17 & 117.12 & 117.28 & 117.32 & 117.31 & 117.23 & 116.86 & 92.47 & 64.94 \\
\hline 20.00 & 69.26 & 114.90 & 120.03 & 120.19 & 120.23 & 120.22 & 120.14 & 119.76 & 94.19 & 63.83 \\
\hline 21.00 & 69.54 & 115.17 & 120.30 & 120.47 & 120.51 & 120.50 & 120.41 & 120.03 & 94.44 & 63.39 \\
\hline 22.00 & 63.08 & 65.50 & 67.02 & 67.18 & 67.24 & 67.23 & 67.10 & 66.63 & 62.97 & 59.81 \\
\hline 22.50 & 61.10 & 58.84 & 59.03 & 59.16 & 59.22 & 59.20 & 59.07 & 58.85 & 58.75 & 60.90 \\
\hline
\end{tabular}


Table B - 3.Temperature $\left({ }^{\circ} \mathrm{C}\right)$ map of the east side of plate 1B5 at BOC 142B (0 EFPD).

\begin{tabular}{|c||c|r|r|r|r|r|r|r|r|c|}
\hline \multirow{2}{*}{$\begin{array}{l}\text { Length of } \\
\text { Plate (in) }\end{array}$} & \multicolumn{10}{|c|}{ Width of Plate (inches) } \\
\hline 22.625 & 60.50 & 59.94 & 60.18 & 60.34 & 60.41 & 60.40 & 60.25 & 60.00 & 59.83 & 59.77 \\
\hline 23.00 & 62.88 & 66.81 & 68.38 & 68.57 & 68.63 & 68.63 & 68.48 & 67.99 & 64.23 & 60.72 \\
\hline 24.00 & 70.51 & 118.64 & 123.91 & 124.12 & 124.18 & 124.18 & 124.05 & 123.65 & 97.21 & 65.40 \\
\hline 25.00 & 70.75 & 118.88 & 124.13 & 124.35 & 124.41 & 124.41 & 124.27 & 123.86 & 97.51 & 65.86 \\
\hline 26.00 & 70.68 & 116.77 & 121.86 & 122.08 & 122.15 & 122.14 & 122.01 & 121.59 & 96.36 & 66.67 \\
\hline 27.00 & 70.92 & 117.09 & 122.19 & 122.42 & 122.49 & 122.48 & 122.34 & 121.92 & 96.84 & 67.16 \\
\hline 28.00 & 71.07 & 116.85 & 121.91 & 122.14 & 122.21 & 122.21 & 122.06 & 121.63 & 96.64 & 66.92 \\
\hline 29.00 & 71.30 & 117.21 & 122.27 & 122.51 & 122.58 & 122.57 & 122.42 & 121.98 & 96.88 & 66.48 \\
\hline 30.00 & 71.38 & 116.58 & 121.58 & 121.82 & 121.90 & 121.89 & 121.73 & 121.29 & 96.57 & 66.37 \\
\hline 31.00 & 71.56 & 116.89 & 121.89 & 122.13 & 122.21 & 122.21 & 122.04 & 121.59 & 96.90 & 66.61 \\
\hline 32.00 & 71.41 & 115.10 & 119.95 & 120.20 & 120.28 & 120.27 & 120.10 & 119.65 & 95.82 & 66.46 \\
\hline 33.00 & 71.59 & 115.42 & 120.28 & 120.53 & 120.61 & 120.60 & 120.43 & 119.97 & 96.10 & 66.52 \\
\hline 34.00 & 71.24 & 112.49 & 117.10 & 117.35 & 117.44 & 117.43 & 117.25 & 116.79 & 94.28 & 66.60 \\
\hline 35.00 & 71.38 & 112.76 & 117.37 & 117.63 & 117.72 & 117.71 & 117.53 & 117.07 & 94.67 & 67.36 \\
\hline 36.00 & 70.83 & 108.81 & 113.10 & 113.36 & 113.45 & 113.44 & 113.25 & 112.79 & 92.30 & 67.45 \\
\hline 37.00 & 70.93 & 109.06 & 113.35 & 113.61 & 113.70 & 113.70 & 113.50 & 113.04 & 92.40 & 67.15 \\
\hline 38.00 & 70.28 & 104.71 & 108.63 & 108.90 & 108.99 & 108.98 & 108.78 & 108.32 & 89.55 & 66.11 \\
\hline 39.00 & 70.34 & 104.93 & 108.85 & 109.12 & 109.21 & 109.20 & 109.00 & 108.54 & 89.75 & 65.94 \\
\hline 40.00 & 69.59 & 99.99 & 103.49 & 103.76 & 103.86 & 103.85 & 103.64 & 103.18 & 86.65 & 65.58 \\
\hline 41.00 & 69.59 & 100.17 & 103.67 & 103.94 & 104.04 & 104.03 & 103.82 & 103.37 & 86.83 & 65.67 \\
\hline 42.00 & 68.63 & 94.33 & 97.33 & 97.60 & 97.70 & 97.69 & 97.47 & 97.02 & 83.15 & 64.86 \\
\hline 43.00 & 68.58 & 94.46 & 97.46 & 97.73 & 97.83 & 97.82 & 97.60 & 97.16 & 83.28 & 65.04 \\
\hline 44.00 & 64.46 & 67.35 & 67.97 & 68.23 & 68.35 & 68.33 & 68.08 & 67.66 & 66.58 & 62.86 \\
\hline 45.00 & 63.71 & 66.81 & 67.28 & 67.52 & 67.63 & 67.62 & 67.37 & 67.02 & 66.25 & 62.53 \\
\hline
\end{tabular}


Table B - 4. Temperature $\left({ }^{\circ} \mathrm{C}\right)$ map of the west side of plate 1B5 at BOC $142 \mathrm{~B}(0 \mathrm{EFPD})$.

\begin{tabular}{|c||c|c|c|r|r|r|r|r|r|r|}
\hline \multirow{2}{*}{$\begin{array}{c}\text { Length of } \\
\text { Plate (in) }\end{array}$} & \multicolumn{10}{|c|}{ Width of Plate (inches) } \\
\hline 22.625 & 61.02 & 58.91 & 59.12 & 59.25 & 59.31 & 59.29 & 59.16 & 58.93 & 58.78 & 60.21 \\
\hline 23.00 & 63.25 & 65.80 & 67.33 & 67.50 & 67.55 & 67.54 & 67.41 & 66.95 & 63.20 & 59.94 \\
\hline 24.00 & 70.22 & 117.29 & 122.52 & 122.70 & 122.74 & 122.74 & 122.63 & 122.25 & 95.96 & 63.86 \\
\hline 25.00 & 70.46 & 117.48 & 122.69 & 122.87 & 122.92 & 122.91 & 122.80 & 122.41 & 96.22 & 64.73 \\
\hline 26.00 & 70.38 & 115.30 & 120.36 & 120.55 & 120.59 & 120.59 & 120.47 & 120.07 & 95.02 & 66.49 \\
\hline 27.00 & 70.58 & 115.58 & 120.64 & 120.83 & 120.88 & 120.87 & 120.75 & 120.34 & 95.43 & 67.16 \\
\hline 28.00 & 70.70 & 115.28 & 120.30 & 120.49 & 120.55 & 120.54 & 120.41 & 120.00 & 95.20 & 66.53 \\
\hline 29.00 & 70.91 & 115.57 & 120.59 & 120.79 & 120.85 & 120.84 & 120.71 & 120.28 & 95.38 & 65.30 \\
\hline 30.00 & 70.94 & 114.89 & 119.85 & 120.05 & 120.10 & 120.09 & 119.96 & 119.53 & 95.01 & 64.83 \\
\hline 31.00 & 71.09 & 115.14 & 120.10 & 120.30 & 120.36 & 120.35 & 120.21 & 119.78 & 95.29 & 64.99 \\
\hline 32.00 & 70.90 & 113.28 & 118.09 & 118.30 & 118.36 & 118.34 & 118.20 & 117.76 & 94.15 & 64.67 \\
\hline 33.00 & 71.03 & 113.52 & 118.33 & 118.54 & 118.60 & 118.59 & 118.44 & 118.00 & 94.37 & 64.75 \\
\hline 34.00 & 70.66 & 110.54 & 115.10 & 115.31 & 115.38 & 115.37 & 115.21 & 114.76 & 92.50 & 65.34 \\
\hline 35.00 & 70.74 & 110.76 & 115.32 & 115.54 & 115.60 & 115.59 & 115.43 & 114.98 & 92.83 & 66.84 \\
\hline 36.00 & 70.17 & 106.72 & 110.96 & 111.18 & 111.25 & 111.23 & 111.06 & 110.62 & 90.43 & 67.35 \\
\hline 37.00 & 70.21 & 106.92 & 111.16 & 111.38 & 111.45 & 111.43 & 111.26 & 110.81 & 90.48 & 66.65 \\
\hline 38.00 & 69.55 & 102.48 & 106.35 & 106.57 & 106.64 & 106.62 & 106.45 & 106.00 & 87.63 & 64.86 \\
\hline 39.00 & 69.55 & 102.64 & 106.51 & 106.74 & 106.81 & 106.79 & 106.61 & 106.17 & 87.77 & 64.31 \\
\hline 40.00 & 68.80 & 97.62 & 101.07 & 101.29 & 101.36 & 101.35 & 101.16 & 100.72 & 84.67 & 63.94 \\
\hline 41.00 & 68.73 & 97.75 & 101.20 & 101.42 & 101.49 & 101.48 & 101.29 & 100.85 & 84.80 & 64.15 \\
\hline 42.00 & 67.78 & 91.81 & 94.75 & 94.97 & 95.04 & 95.02 & 94.83 & 94.40 & 81.06 & 63.30 \\
\hline 43.00 & 67.66 & 91.90 & 94.84 & 95.06 & 95.13 & 95.11 & 94.92 & 94.50 & 81.15 & 63.81 \\
\hline 44.00 & 63.95 & 65.44 & 66.01 & 66.24 & 66.33 & 66.31 & 66.08 & 65.68 & 64.64 & 62.52 \\
\hline 45.00 & 63.24 & 64.90 & 65.31 & 65.52 & 65.61 & 65.58 & 65.36 & 65.03 & 64.29 & 62.37 \\
\hline
\end{tabular}


Table B - 5. Temperature $\left({ }^{\circ} \mathrm{C}\right)$ map of the east side of plate 1T2 at MOC 142B (16 EFPD).

\begin{tabular}{|c||c|c|c|c|c|c|c|c|c|c|}
\hline \multirow{2}{*}{$\begin{array}{c}\text { Length of } \\
\text { Plate (in) }\end{array}$} & \multicolumn{10}{|c|}{ Width of Plate (inches) } \\
\hline & 0.05 & 0.25 & 0.50 & 0.75 & 1.00 & 1.25 & 1.50 & 1.75 & 2.00 & 2.24 \\
\hline 0.125 & 52.10 & 51.80 & 51.77 & 51.77 & 51.77 & 51.77 & 51.77 & 51.78 & 51.86 & 52.14 \\
\hline 1.00 & 53.44 & 52.41 & 52.50 & 52.52 & 52.52 & 52.52 & 52.51 & 52.46 & 52.43 & 53.31 \\
\hline 2.00 & 57.53 & 76.63 & 78.91 & 78.96 & 78.96 & 78.96 & 78.96 & 78.82 & 67.49 & 55.83 \\
\hline 3.00 & 58.00 & 76.91 & 79.20 & 79.26 & 79.26 & 79.26 & 79.25 & 79.10 & 67.80 & 56.13 \\
\hline 4.00 & 59.23 & 82.82 & 85.56 & 85.63 & 85.63 & 85.63 & 85.62 & 85.45 & 71.74 & 57.06 \\
\hline 5.00 & 59.61 & 83.06 & 85.80 & 85.87 & 85.88 & 85.88 & 85.86 & 85.68 & 72.00 & 57.33 \\
\hline 6.00 & 60.97 & 89.32 & 92.51 & 92.58 & 92.59 & 92.59 & 92.57 & 92.38 & 76.22 & 58.20 \\
\hline 7.00 & 61.37 & 89.60 & 92.79 & 92.87 & 92.89 & 92.89 & 92.86 & 92.66 & 76.53 & 58.49 \\
\hline 8.00 & 62.49 & 94.41 & 97.93 & 98.02 & 98.03 & 98.03 & 98.00 & 97.79 & 79.86 & 59.24 \\
\hline 9.00 & 62.83 & 94.67 & 98.19 & 98.28 & 98.30 & 98.30 & 98.26 & 98.04 & 80.33 & 60.47 \\
\hline 10.00 & 63.80 & 98.56 & 102.60 & 102.71 & 102.74 & 102.73 & 102.69 & 102.42 & 82.98 & 60.92 \\
\hline 11.00 & 64.15 & 98.87 & 102.92 & 103.04 & 103.06 & 103.06 & 103.01 & 102.73 & 83.18 & 60.74 \\
\hline 12.00 & 65.01 & 102.06 & 106.33 & 106.46 & 106.49 & 106.49 & 106.43 & 106.13 & 85.32 & 61.16 \\
\hline 13.00 & 65.35 & 102.38 & 106.66 & 106.80 & 106.83 & 106.83 & 106.77 & 106.46 & 85.65 & 61.53 \\
\hline 14.00 & 66.11 & 105.40 & 109.90 & 110.04 & 110.08 & 110.08 & 110.01 & 109.69 & 87.68 & 62.15 \\
\hline 15.00 & 66.46 & 105.74 & 110.24 & 110.39 & 110.43 & 110.43 & 110.35 & 110.02 & 87.97 & 62.36 \\
\hline 16.00 & 67.11 & 107.82 & 112.46 & 112.62 & 112.66 & 112.66 & 112.58 & 112.23 & 89.40 & 62.72 \\
\hline 17.00 & 67.44 & 108.16 & 112.80 & 112.97 & 113.01 & 113.01 & 112.92 & 112.57 & 89.83 & 63.89 \\
\hline 18.00 & 67.79 & 108.91 & 113.59 & 113.76 & 113.81 & 113.81 & 113.71 & 113.35 & 90.53 & 64.54 \\
\hline 19.00 & 68.11 & 109.24 & 113.92 & 114.10 & 114.15 & 114.15 & 114.05 & 113.68 & 90.73 & 64.58 \\
\hline 20.00 & 68.75 & 111.65 & 116.48 & 116.67 & 116.72 & 116.72 & 116.61 & 116.23 & 92.25 & 64.37 \\
\hline 21.00 & 69.06 & 111.96 & 116.80 & 117.00 & 117.05 & 117.05 & 116.94 & 116.55 & 92.54 & 64.37 \\
\hline 22.00 & 62.37 & 65.64 & 67.08 & 67.25 & 67.32 & 67.31 & 67.17 & 66.71 & 63.33 & 60.33 \\
\hline 22.50 & 60.16 & 59.52 & 59.72 & 59.87 & 59.93 & 59.93 & 59.78 & 59.56 & 59.49 & 59.75 \\
\hline
\end{tabular}


Table B - 6. Temperature $\left({ }^{\circ} \mathrm{C}\right)$ map of the west side of plate 1T2 at MOC 142B (16 EFPD).

\begin{tabular}{|c||c|c|c|c|c|c|c|c|c|c|}
\hline \multirow{2}{*}{$\begin{array}{c}\text { Length of } \\
\text { Plate (in) }\end{array}$} & \multicolumn{10}{|c|}{ Width of Plate (inches) } \\
\hline & 0.05 & 0.25 & 0.50 & 0.75 & 1.00 & 1.25 & 1.50 & 1.75 & 2.00 & 2.24 \\
\hline 0.125 & 52.36 & 51.80 & 51.77 & 51.77 & 51.77 & 51.77 & 51.77 & 51.78 & 51.85 & 52.34 \\
\hline 1.00 & 53.82 & 52.41 & 52.50 & 52.51 & 52.51 & 52.51 & 52.51 & 52.46 & 52.42 & 53.57 \\
\hline 2.00 & 57.38 & 76.61 & 78.90 & 78.94 & 78.95 & 78.95 & 78.94 & 78.80 & 67.48 & 55.42 \\
\hline 3.00 & 57.88 & 76.86 & 79.15 & 79.20 & 79.21 & 79.21 & 79.20 & 79.05 & 67.75 & 55.54 \\
\hline 4.00 & 59.04 & 82.74 & 85.48 & 85.54 & 85.54 & 85.54 & 85.53 & 85.36 & 71.67 & 56.39 \\
\hline 5.00 & 59.44 & 82.93 & 85.68 & 85.74 & 85.74 & 85.74 & 85.73 & 85.55 & 71.89 & 56.56 \\
\hline 6.00 & 60.72 & 89.15 & 92.34 & 92.40 & 92.41 & 92.41 & 92.40 & 92.21 & 76.07 & 57.33 \\
\hline 7.00 & 61.15 & 89.38 & 92.56 & 92.64 & 92.65 & 92.64 & 92.63 & 92.43 & 76.32 & 57.64 \\
\hline 8.00 & 62.23 & 94.14 & 97.65 & 97.73 & 97.74 & 97.74 & 97.72 & 97.51 & 79.61 & 58.30 \\
\hline 9.00 & 62.60 & 94.34 & 97.85 & 97.94 & 97.95 & 97.95 & 97.92 & 97.71 & 80.02 & 60.52 \\
\hline 10.00 & 63.54 & 98.19 & 102.15 & 102.25 & 102.27 & 102.27 & 102.23 & 101.97 & 82.63 & 60.67 \\
\hline 11.00 & 63.90 & 98.41 & 102.40 & 102.51 & 102.53 & 102.52 & 102.49 & 102.21 & 82.78 & 59.93 \\
\hline 12.00 & 64.73 & 101.48 & 105.75 & 105.86 & 105.88 & 105.88 & 105.83 & 105.55 & 84.87 & 59.91 \\
\hline 13.00 & 65.09 & 101.74 & 106.01 & 106.13 & 106.15 & 106.14 & 106.10 & 105.80 & 85.14 & 60.21 \\
\hline 14.00 & 65.83 & 104.70 & 109.18 & 109.31 & 109.33 & 109.33 & 109.28 & 108.97 & 87.12 & 60.91 \\
\hline 15.00 & 66.18 & 104.97 & 109.45 & 109.58 & 109.61 & 109.61 & 109.55 & 109.23 & 87.36 & 61.17 \\
\hline 16.00 & 66.81 & 106.98 & 111.60 & 111.73 & 111.76 & 111.76 & 111.70 & 111.37 & 88.73 & 61.47 \\
\hline 17.00 & 67.14 & 107.25 & 111.87 & 112.01 & 112.04 & 112.04 & 111.97 & 111.63 & 89.10 & 63.57 \\
\hline 18.00 & 67.49 & 107.93 & 112.59 & 112.73 & 112.77 & 112.76 & 112.69 & 112.34 & 89.74 & 64.54 \\
\hline 19.00 & 67.81 & 108.20 & 112.85 & 113.00 & 113.04 & 113.03 & 112.95 & 112.60 & 89.88 & 64.41 \\
\hline 20.00 & 68.41 & 110.53 & 115.34 & 115.50 & 115.53 & 115.53 & 115.44 & 115.08 & 91.35 & 63.30 \\
\hline 21.00 & 68.67 & 110.78 & 115.59 & 115.75 & 115.79 & 115.79 & 115.70 & 115.33 & 91.58 & 62.86 \\
\hline 22.00 & 62.72 & 64.71 & 66.11 & 66.27 & 66.32 & 66.31 & 66.19 & 65.75 & 62.38 & 59.57 \\
\hline 22.50 & 60.89 & 58.55 & 58.71 & 58.84 & 58.89 & 58.88 & 58.76 & 58.55 & 58.49 & 60.71 \\
\hline
\end{tabular}


Table B - 7. Temperature $\left({ }^{\circ} \mathrm{C}\right)$ map of the east side of plate 1B5 at MOC 142B (16 EFPD).

\begin{tabular}{|c||c|c|c|c|c|c|c|c|c|c|}
\hline \multirow{2}{*}{$\begin{array}{l}\text { Length of } \\
\text { Plate (in) }\end{array}$} & 0.05 & 0.25 & 0.50 & 0.75 & 1.00 & 1.25 & 1.50 & 1.75 & 2.00 & 2.24 \\
\hline 22.625 & 60.26 & 59.59 & 59.80 & 59.95 & 60.02 & 60.01 & 59.87 & 59.63 & 59.51 & 59.58 \\
\hline 23.00 & 62.40 & 65.76 & 67.18 & 67.36 & 67.42 & 67.41 & 67.27 & 66.82 & 63.45 & 60.38 \\
\hline 24.00 & 69.26 & 112.61 & 117.43 & 117.63 & 117.69 & 117.69 & 117.57 & 117.18 & 93.10 & 64.59 \\
\hline 25.00 & 69.52 & 112.90 & 117.73 & 117.94 & 118.00 & 117.99 & 117.87 & 117.47 & 93.44 & 65.05 \\
\hline 26.00 & 69.60 & 111.92 & 116.66 & 116.87 & 116.93 & 116.93 & 116.79 & 116.39 & 93.02 & 65.91 \\
\hline 27.00 & 69.82 & 112.22 & 116.97 & 117.19 & 117.25 & 117.25 & 117.11 & 116.70 & 93.47 & 66.38 \\
\hline 28.00 & 70.03 & 112.42 & 117.16 & 117.38 & 117.45 & 117.45 & 117.30 & 116.89 & 93.56 & 66.19 \\
\hline 29.00 & 70.24 & 112.75 & 117.49 & 117.72 & 117.79 & 117.78 & 117.64 & 117.22 & 93.77 & 65.76 \\
\hline 30.00 & 70.22 & 111.55 & 116.18 & 116.41 & 116.48 & 116.48 & 116.33 & 115.90 & 93.08 & 65.59 \\
\hline 31.00 & 70.39 & 111.83 & 116.47 & 116.70 & 116.78 & 116.77 & 116.61 & 116.19 & 93.38 & 65.82 \\
\hline 32.00 & 70.33 & 110.71 & 115.24 & 115.48 & 115.55 & 115.55 & 115.39 & 114.96 & 92.74 & 65.72 \\
\hline 33.00 & 70.49 & 111.01 & 115.55 & 115.79 & 115.87 & 115.86 & 115.70 & 115.26 & 93.00 & 65.78 \\
\hline 34.00 & 70.31 & 109.10 & 113.48 & 113.72 & 113.80 & 113.79 & 113.62 & 113.18 & 91.86 & 65.95 \\
\hline 35.00 & 70.43 & 109.36 & 113.74 & 113.98 & 114.07 & 114.06 & 113.88 & 113.45 & 92.22 & 66.68 \\
\hline 36.00 & 69.92 & 105.70 & 109.77 & 110.02 & 110.10 & 110.09 & 109.91 & 109.47 & 90.05 & 66.77 \\
\hline 37.00 & 70.01 & 105.94 & 110.01 & 110.26 & 110.34 & 110.34 & 110.15 & 109.71 & 90.14 & 66.47 \\
\hline 38.00 & 69.53 & 102.47 & 106.25 & 106.50 & 106.59 & 106.58 & 106.39 & 105.95 & 87.88 & 65.57 \\
\hline 39.00 & 69.58 & 102.69 & 106.46 & 106.72 & 106.81 & 106.80 & 106.60 & 106.17 & 88.07 & 65.40 \\
\hline 40.00 & 68.88 & 98.12 & 101.50 & 101.76 & 101.85 & 101.84 & 101.64 & 101.21 & 85.25 & 65.06 \\
\hline 41.00 & 68.87 & 98.29 & 101.68 & 101.93 & 102.03 & 102.02 & 101.82 & 101.39 & 85.41 & 65.15 \\
\hline 42.00 & 68.09 & 93.44 & 96.41 & 96.66 & 96.76 & 96.75 & 96.54 & 96.12 & 82.37 & 64.46 \\
\hline 43.00 & 68.05 & 93.58 & 96.55 & 96.80 & 96.89 & 96.88 & 96.68 & 96.26 & 82.50 & 64.64 \\
\hline 44.00 & 63.93 & 66.51 & 67.10 & 67.35 & 67.45 & 67.43 & 67.20 & 66.81 & 65.79 & 62.44 \\
\hline 45.00 & 63.17 & 65.98 & 66.40 & 66.63 & 66.73 & 66.72 & 66.49 & 66.16 & 65.46 & 62.09 \\
\hline
\end{tabular}


Table B - 8. Temperature $\left({ }^{\circ} \mathrm{C}\right)$ map of the west side of plate 1B5 at MOC 142B (16 EFPD).

\begin{tabular}{|c|c|c|c|c|c|c|c|c|c|c|}
\hline \multirow{2}{*}{$\begin{array}{l}\text { Length of } \\
\text { Plate (in) }\end{array}$} & \multicolumn{10}{|c|}{ Width of Plate (inches) } \\
\hline & 0.05 & 0.25 & 0.50 & 0.75 & 1.00 & 1.25 & 1.50 & 1.75 & 2.00 & 2.24 \\
\hline 22.625 & 60.82 & 58.62 & 58.79 & 58.92 & 58.97 & 58.96 & 58.83 & 58.62 & 58.51 & 60.04 \\
\hline 23.00 & 62.82 & 64.81 & 66.19 & 66.34 & 66.40 & 66.39 & 66.26 & 65.83 & 62.47 & 59.66 \\
\hline 24.00 & 69.08 & 111.30 & 116.10 & 116.26 & 116.31 & 116.30 & 116.20 & 115.84 & 92.04 & 63.20 \\
\hline 25.00 & 69.33 & 111.56 & 116.35 & 116.52 & 116.57 & 116.56 & 116.45 & 116.08 & 92.31 & 64.06 \\
\hline 26.00 & 69.39 & 110.52 & 115.22 & 115.40 & 115.45 & 115.44 & 115.33 & 114.94 & 91.85 & 65.80 \\
\hline 27.00 & 69.58 & 110.78 & 115.49 & 115.67 & 115.72 & 115.71 & 115.60 & 115.21 & 92.22 & 66.45 \\
\hline 28.00 & 69.76 & 110.93 & 115.63 & 115.82 & 115.87 & 115.86 & 115.74 & 115.34 & 92.27 & 65.88 \\
\hline 29.00 & 69.94 & 111.20 & 115.91 & 116.10 & 116.15 & 116.14 & 116.01 & 115.61 & 92.41 & 64.69 \\
\hline 30.00 & 69.88 & 109.94 & 114.54 & 114.73 & 114.78 & 114.77 & 114.64 & 114.24 & 91.68 & 64.19 \\
\hline 31.00 & 70.01 & 110.18 & 114.78 & 114.97 & 115.02 & 115.01 & 114.88 & 114.47 & 91.92 & 64.34 \\
\hline 32.00 & 69.92 & 109.00 & 113.49 & 113.69 & 113.74 & 113.73 & 113.60 & 113.18 & 91.24 & 64.07 \\
\hline 33.00 & 70.04 & 109.25 & 113.74 & 113.94 & 114.00 & 113.99 & 113.85 & 113.43 & 91.43 & 64.24 \\
\hline 34.00 & 69.82 & 107.27 & 111.60 & 111.80 & 111.86 & 111.85 & 111.70 & 111.28 & 90.26 & 64.80 \\
\hline 35.00 & 69.89 & 107.49 & 111.82 & 112.02 & 112.08 & 112.07 & 111.92 & 111.49 & 90.56 & 66.23 \\
\hline 36.00 & 69.35 & 103.75 & .77 & 107.98 & 04 & 108.02 & 107.87 & 10 & & 66.72 \\
\hline 37.00 & 69.39 & 103.93 & 107.96 & 108.17 & 108.23 & 108.22 & 108.06 & 107.63 & 88.42 & 66.04 \\
\hline 38.00 & 68.87 & 100.39 & 104.12 & 104.33 & 104.39 & 104.38 & 104.21 & 103.79 & 86.14 & 64.40 \\
\hline 39.00 & 68.86 & 100.56 & 104.29 & 104.49 & 104.56 & 104.54 & 104.38 & 103.96 & 86.28 & 63.87 \\
\hline 40.00 & 68.16 & 95.91 & 99.24 & 99.45 & 99.52 & 99.50 & 99.33 & 98.91 & 83.42 & 63.52 \\
\hline 41.00 & 68.09 & 96.04 & 99.37 & 99.58 & 99.65 & 99.63 & 99.46 & 99.04 & 83.54 & 63.72 \\
\hline 42.00 & 67.29 & 91.10 & 94.01 & 94.21 & 94.28 & 94.27 & 94.08 & 93.68 & 80.43 & 62.97 \\
\hline 43.00 & 67.18 & 91.20 & 94.10 & 94.31 & 94.38 & 94.36 & 94.18 & 93.78 & 80.53 & 63.47 \\
\hline 44.00 & 63.46 & 64.74 & 65.28 & 65.49 & 65.58 & 65.55 & 65.34 & 64.97 & 63.99 & 62.13 \\
\hline 45.00 & 62.75 & 64.19 & 64.57 & 64.77 & 64.85 & 64.83 & 64.62 & 64.31 & 63.64 & 61.95 \\
\hline
\end{tabular}


Table B - 9. Temperature $\left({ }^{\circ} \mathrm{C}\right)$ map of the east side of plate 1T2 at MOC 142B (29.7 EFPD).

\begin{tabular}{|c||c|c|c|c|c|c|c|c|c|c|}
\hline \multirow{2}{*}{$\begin{array}{c}\text { Length of } \\
\text { Plate (in) }\end{array}$} & \multicolumn{10}{|c|}{ Width of Plate (inches) } \\
\hline & 0.05 & 0.25 & 0.50 & 0.75 & 1.00 & 1.25 & 1.50 & 1.75 & 2.00 & 2.24 \\
\hline 0.125 & 52.10 & 51.80 & 51.77 & 51.77 & 51.77 & 51.77 & 51.77 & 51.78 & 51.86 & 52.14 \\
\hline 1.00 & 53.56 & 52.49 & 52.61 & 52.63 & 52.63 & 52.63 & 52.63 & 52.56 & 52.50 & 53.40 \\
\hline 2.00 & 58.26 & 80.29 & 82.86 & 82.91 & 82.92 & 82.92 & 82.91 & 82.76 & 69.90 & 56.32 \\
\hline 3.00 & 58.76 & 80.59 & 83.18 & 83.24 & 83.24 & 83.24 & 83.23 & 83.06 & 70.23 & 56.64 \\
\hline 4.00 & 60.07 & 86.84 & 89.88 & 89.95 & 89.95 & 89.95 & 89.94 & 89.76 & 74.44 & 57.63 \\
\hline 5.00 & 60.47 & 87.10 & 90.14 & 90.21 & 90.22 & 90.22 & 90.21 & 90.02 & 74.72 & 57.91 \\
\hline 6.00 & 61.91 & 93.76 & 97.26 & 97.34 & 97.35 & 97.35 & 97.33 & 97.13 & 79.27 & 58.83 \\
\hline 7.00 & 62.34 & 94.07 & 97.57 & 97.66 & 97.67 & 97.67 & 97.65 & 97.43 & 79.59 & 59.13 \\
\hline 8.00 & 63.50 & 99.04 & 103.14 & 103.25 & 103.27 & 103.27 & 103.23 & 102.97 & 83.07 & 59.91 \\
\hline 9.00 & 63.87 & 99.36 & 103.47 & 103.59 & 103.61 & 103.61 & 103.57 & 103.29 & 83.57 & 61.21 \\
\hline 10.00 & 64.92 & 103.92 & 108.38 & 108.50 & 108.53 & 108.53 & 108.48 & 108.18 & 86.49 & 61.70 \\
\hline 11.00 & 65.29 & 104.26 & 108.72 & 108.86 & 108.88 & 108.88 & 108.83 & 108.52 & 86.72 & 61.50 \\
\hline 12.00 & 66.12 & 107.16 & 111.82 & 111.96 & 111.99 & 111.99 & 111.93 & 111.61 & 88.68 & 61.90 \\
\hline 13.00 & 66.48 & 107.51 & 112.17 & 112.32 & 112.36 & 112.36 & 112.29 & 111.96 & 89.03 & 62.28 \\
\hline 14.00 & 67.16 & 110.08 & 114.92 & 115.08 & 115.12 & 115.11 & 115.04 & 114.69 & 90.77 & 62.86 \\
\hline 15.00 & 67.53 & 110.44 & 115.28 & 115.45 & 115.49 & 115.48 & 115.40 & 115.05 & 91.08 & 63.08 \\
\hline 16.00 & 68.10 & 111.95 & 116.88 & 117.06 & 117.10 & 117.10 & 117.01 & 116.64 & 92.15 & 63.39 \\
\hline 17.00 & 68.44 & 112.30 & 117.24 & 117.42 & 117.47 & 117.46 & 117.37 & 116.99 & 92.60 & 64.60 \\
\hline 18.00 & 68.93 & 113.84 & 118.87 & 119.06 & 119.11 & 119.10 & 119.00 & 118.62 & 93.81 & 65.36 \\
\hline 19.00 & 69.28 & 114.20 & 119.23 & 119.43 & 119.48 & 119.48 & 119.37 & 118.97 & 94.03 & 65.41 \\
\hline 20.00 & 69.87 & 116.15 & 121.30 & 121.50 & 121.56 & 121.55 & 121.44 & 121.03 & 95.34 & 65.13 \\
\hline 21.00 & 70.18 & 116.48 & 121.63 & 121.84 & 121.90 & 121.90 & 121.77 & 121.36 & 95.67 & 65.12 \\
\hline 22.00 & 63.00 & 66.83 & 68.39 & 68.58 & 68.65 & 68.65 & 68.49 & 67.99 & 64.31 & 60.79 \\
\hline 22.50 & 60.63 & 60.27 & 60.51 & 60.68 & 60.75 & 60.74 & 60.59 & 60.33 & 60.18 & 60.14 \\
\hline
\end{tabular}


Table B - 10. Temperature $\left({ }^{\circ} \mathrm{C}\right)$ map of the west side of plate 1T2 at MOC 142B (29.7 EFPD).

\begin{tabular}{|c|c|c|c|c|c|c|c|c|c|c|}
\hline \multirow{2}{*}{$\begin{array}{l}\text { Length of } \\
\text { Plate (in) }\end{array}$} & \multicolumn{10}{|c|}{ Width of Plate (inches) } \\
\hline & 0.05 & 0.25 & 0.50 & 0.75 & 1.00 & 1.25 & 1.50 & 1.75 & 2.00 & 2.24 \\
\hline 0.125 & 52.36 & 51.80 & 51.77 & 51.77 & 51.77 & 51.77 & 51.77 & 51.78 & 51.86 & 52.34 \\
\hline 1.00 & 53.91 & 52.49 & 52.61 & 52.63 & 52.63 & 52.63 & 52.62 & 52.56 & 52.49 & 53.65 \\
\hline 2.00 & 58.01 & 80.27 & 82.84 & 82.89 & 82.89 & 82.89 & 82.89 & 82.74 & 69.88 & 55.81 \\
\hline 3.00 & 58.54 & 80.53 & 83.12 & 83.18 & 83.18 & 83.18 & 83.17 & 83.00 & 70.18 & 55.94 \\
\hline 4.00 & 59.77 & 86.74 & 89.78 & 89.84 & 89.85 & 89.85 & 89.84 & 89.66 & 74.36 & 56.84 \\
\hline 5.00 & 60.19 & 86.95 & 89.99 & 90.06 & 90.07 & 90.07 & 90.05 & 89.87 & 74.59 & 57.02 \\
\hline 6.00 & 61.56 & 93.57 & 97.06 & 97.14 & 97.15 & 97.14 & 97.13 & 96.93 & 79.09 & 57.83 \\
\hline 7.00 & 62.00 & 93.81 & 97.31 & 97.39 & 97.40 & 97.40 & 97.38 & 97.17 & 79.36 & 58.15 \\
\hline 8.00 & 63.12 & 98.73 & 102.77 & 102.87 & 102.88 & 102.88 & 102.85 & 102.59 & 82.78 & 58.84 \\
\hline 9.00 & 63.52 & 98.95 & 103.03 & 103.13 & 103.15 & 103.15 & 103.12 & 102.85 & 83.21 & 61.18 \\
\hline 10.00 & 64.54 & 103.42 & 107.87 & 107.98 & 108.00 & 107.99 & 107.96 & 107.67 & 86.10 & 61.35 \\
\hline 11.00 & 64.93 & 103.69 & 10 & 108.25 & 10 & 10 & 108.23 & 107.93 & 86 & 60.56 \\
\hline 12.00 & 65.72 & 106.51 & 111.15 & 111.28 & 111.30 & 111.30 & 111.25 & 110.94 & 88.16 & 60.50 \\
\hline 13.00 & 66.09 & 106.78 & 111.43 & 111.56 & 111.59 & 111.58 & 111.53 & 111.21 & 88.45 & 60.82 \\
\hline 14.00 & 66.78 & 109.28 & 114.11 & 114.24 & 114.27 & 114.27 & 114.21 & 113.88 & 90.14 & 61.49 \\
\hline 15.00 & 67.15 & 109.57 & 114.39 & 114.53 & 114.56 & 114.56 & 114.50 & 114.15 & 90.39 & 61.76 \\
\hline 16.00 & 67.69 & 111.00 & 115.91 & 116.06 & 116.09 & 116.09 & 116.02 & 115.66 & 91.39 & 62.02 \\
\hline 17.00 & 68.04 & 111.28 & 116.19 & 116.34 & 116.38 & 116.37 & 116.30 & 115.94 & 91.78 & 64.20 \\
\hline 18.00 & 68.52 & 112.74 & 117.75 & 117.91 & 117.95 & 117.94 & 117.86 & 117.49 & 92.92 & 65.29 \\
\hline 19.00 & 68.86 & 113.03 & 118.04 & 118.20 & 118.24 & 118.24 & 118.15 & 117.77 & 93.08 & 65.16 \\
\hline 20.00 & 69.42 & 114.90 & 120.02 & 120.20 & 120.24 & 120.23 & 120.14 & 119.75 & 94.26 & 63.95 \\
\hline 21.00 & 69.68 & 115.16 & 120.29 & 120.46 & 120.51 & 120.50 & 120.40 & 120.01 & 94.51 & 63.48 \\
\hline 22.00 & 63.28 & 65.78 & 67.31 & 67.48 & 67.54 & 67.53 & 67.39 & 66.91 & 63.24 & 59.94 \\
\hline 22.50 & 61.31 & 59.17 & 59.38 & 59.52 & 59.58 & 59.57 & 59.43 & 59.19 & 59.06 & 61.09 \\
\hline
\end{tabular}


Table B - 11. Temperature $\left({ }^{\circ} \mathrm{C}\right)$ map of the east side of plate 1B5 at MOC 142B (29.7 EFPD).

\begin{tabular}{|c||c|c|c|c|c|c|c|c|c|c|}
\hline \multirow{2}{*}{$\begin{array}{l}\text { Length of } \\
\text { Plate (in) }\end{array}$} & \multicolumn{10}{|c|}{ Width of Plate (inches) } \\
\hline 22.625 & 60.74 & 60.34 & 60.60 & 60.77 & 60.84 & 60.83 & 60.67 & 60.41 & 60.21 & 59.96 \\
\hline 23.00 & 63.04 & 66.99 & 68.53 & 68.73 & 68.80 & 68.79 & 68.64 & 68.15 & 64.46 & 60.85 \\
\hline 24.00 & 70.44 & 117.41 & 122.57 & 122.79 & 122.85 & 122.85 & 122.72 & 122.31 & 96.49 & 65.38 \\
\hline 25.00 & 70.72 & 117.74 & 122.89 & 123.12 & 123.18 & 123.18 & 123.04 & 122.62 & 96.86 & 65.87 \\
\hline 26.00 & 70.82 & 116.73 & 121.80 & 122.03 & 122.10 & 122.09 & 121.95 & 121.52 & 96.44 & 66.79 \\
\hline 27.00 & 71.07 & 117.06 & 122.14 & 122.37 & 122.44 & 122.44 & 122.29 & 121.86 & 96.92 & 67.30 \\
\hline 28.00 & 71.33 & 117.48 & 122.57 & 122.80 & 122.88 & 122.87 & 122.72 & 122.28 & 97.16 & 67.11 \\
\hline 29.00 & 71.57 & 117.84 & 122.93 & 123.17 & 123.25 & 123.24 & 123.08 & 122.64 & 97.40 & 66.67 \\
\hline 30.00 & 71.65 & 117.16 & 122.19 & 122.43 & 122.51 & 122.50 & 122.34 & 121.89 & 97.06 & 66.56 \\
\hline 31.00 & 71.83 & 117.47 & 122.49 & 122.74 & 122.82 & 122.82 & 122.65 & 122.19 & 97.39 & 66.81 \\
\hline 32.00 & 71.67 & 115.64 & 120.51 & 120.76 & 120.84 & 120.84 & 120.66 & 120.20 & 96.28 & 66.65 \\
\hline 33.00 & 71.85 & 115.93 & 120.81 & 121.06 & 121.15 & 121.14 & 120.96 & 120.50 & 96.55 & 66.70 \\
\hline 34.00 & 71.69 & 114.05 & 118.76 & 119.02 & 119.11 & 119.10 & 118.92 & 118.45 & 95.42 & 66.91 \\
\hline 35.00 & 71.84 & 114.34 & 119.05 & 119.31 & 119.40 & 119.39 & 119.20 & 118.73 & 95.82 & 67.70 \\
\hline 36.00 & 71.47 & 111.46 & 115.93 & 116.19 & 116.29 & 116.28 & 116.08 & 115.61 & 94.16 & 67.92 \\
\hline 37.00 & 71.59 & 111.72 & 116.20 & 116.47 & 116.56 & 116.55 & 116.35 & 115.88 & 94.27 & 67.61 \\
\hline 38.00 & 71.14 & 108.44 & 112.64 & 112.91 & 113.01 & 113.00 & 112.79 & 112.32 & 92.11 & 66.69 \\
\hline 39.00 & 71.21 & 108.68 & 112.88 & 113.15 & 113.25 & 113.24 & 113.03 & 112.56 & 92.33 & 66.52 \\
\hline 40.00 & 70.46 & 103.59 & 107.37 & 107.64 & 107.74 & 107.73 & 107.52 & 107.04 & 89.13 & 66.17 \\
\hline 41.00 & 70.47 & 103.79 & 107.56 & 107.84 & 107.94 & 107.93 & 107.71 & 107.24 & 89.33 & 66.28 \\
\hline 42.00 & 69.61 & 98.44 & 101.75 & 102.03 & 102.14 & 102.13 & 101.90 & 101.44 & 85.90 & 65.52 \\
\hline 43.00 & 69.58 & 98.59 & 101.91 & 102.19 & 102.29 & 102.28 & 102.06 & 101.59 & 86.06 & 65.72 \\
\hline 44.00 & 64.94 & 68.02 & 68.69 & 68.97 & 69.08 & 69.06 & 68.80 & 68.36 & 67.20 & 63.24 \\
\hline 45.00 & 64.10 & 67.42 & 67.91 & 68.17 & 68.28 & 68.27 & 68.01 & 67.64 & 66.82 & 62.85 \\
\hline
\end{tabular}


Table B - 12. Temperature $\left({ }^{\circ} \mathrm{C}\right)$ map of the west side of plate 1B5 at MOC 142B (29.7 EFPD).

\begin{tabular}{|c||c|r|r|r|r|r|r|r|r|r|}
\hline \multirow{2}{*}{$\begin{array}{c}\text { Length of } \\
\text { Plate (in) }\end{array}$} & \multicolumn{10}{|c|}{ Width of Plate (inches) } \\
\hline 22.625 & 61.24 & 59.24 & 59.46 & 59.60 & 59.67 & 59.65 & 59.51 & 59.27 & 59.08 & 60.39 \\
\hline 23.00 & 63.40 & 65.91 & 67.42 & 67.59 & 67.65 & 67.64 & 67.50 & 67.03 & 63.36 & 60.04 \\
\hline 24.00 & 70.16 & 115.97 & 121.09 & 121.28 & 121.32 & 121.32 & 121.21 & 120.82 & 95.17 & 63.85 \\
\hline 25.00 & 70.44 & 116.25 & 121.36 & 121.55 & 121.60 & 121.59 & 121.48 & 121.08 & 95.48 & 64.75 \\
\hline 26.00 & 70.51 & 115.19 & 120.22 & 120.41 & 120.46 & 120.45 & 120.33 & 119.92 & 95.02 & 66.60 \\
\hline 27.00 & 70.72 & 115.47 & 120.50 & 120.70 & 120.75 & 120.74 & 120.62 & 120.20 & 95.44 & 67.30 \\
\hline 28.00 & 70.94 & 115.83 & 120.88 & 121.08 & 121.13 & 121.12 & 120.99 & 120.57 & 95.65 & 66.71 \\
\hline 29.00 & 71.15 & 116.13 & 121.18 & 121.38 & 121.44 & 121.43 & 121.29 & 120.86 & 95.83 & 65.47 \\
\hline 30.00 & 71.19 & 115.40 & 120.37 & 120.58 & 120.64 & 120.63 & 120.49 & 120.05 & 95.43 & 65.00 \\
\hline 31.00 & 71.34 & 115.65 & 120.63 & 120.84 & 120.90 & 120.89 & 120.74 & 120.30 & 95.71 & 65.16 \\
\hline 32.00 & 71.14 & 113.75 & 118.57 & 118.79 & 118.85 & 118.84 & 118.69 & 118.24 & 94.55 & 64.83 \\
\hline 33.00 & 71.28 & 114.02 & 118.84 & 119.06 & 119.12 & 119.11 & 118.95 & 118.51 & 94.78 & 65.02 \\
\hline 34.00 & 71.08 & 112.03 & 116.69 & 116.91 & 116.98 & 116.96 & 116.80 & 116.35 & 93.57 & 65.61 \\
\hline 35.00 & 71.17 & 112.26 & 116.92 & 117.14 & 117.21 & 117.20 & 117.03 & 116.58 & 93.92 & 67.15 \\
\hline 36.00 & 70.77 & 109.31 & 113.73 & 113.95 & 114.02 & 114.00 & 113.83 & 113.38 & 92.19 & 67.80 \\
\hline 37.00 & 70.82 & 109.52 & 113.94 & 114.16 & 114.24 & 114.22 & 114.05 & 113.59 & 92.26 & 67.08 \\
\hline 38.00 & 70.34 & 106.15 & 110.29 & 110.52 & 110.59 & 110.57 & 110.39 & 109.94 & 90.05 & 65.35 \\
\hline 39.00 & 70.35 & 106.33 & 110.47 & 110.70 & 110.78 & 110.76 & 110.58 & 110.12 & 90.21 & 64.80 \\
\hline 40.00 & 69.58 & 101.15 & 104.87 & 105.10 & 105.17 & 105.15 & 104.96 & 104.51 & 87.02 & 64.43 \\
\hline 41.00 & 69.53 & 101.30 & 105.01 & 105.24 & 105.32 & 105.30 & 105.11 & 104.66 & 87.16 & 64.65 \\
\hline 42.00 & 68.66 & 95.84 & 99.09 & 99.32 & 99.40 & 99.38 & 99.18 & 98.74 & 83.72 & 63.84 \\
\hline 43.00 & 68.56 & 95.95 & 99.20 & 99.43 & 99.51 & 99.49 & 99.29 & 98.85 & 83.84 & 64.39 \\
\hline 44.00 & 64.38 & 66.02 & 66.64 & 66.88 & 66.97 & 66.95 & 66.71 & 66.29 & 65.16 & 62.87 \\
\hline 45.00 & 63.59 & 65.41 & 65.85 & 66.07 & 66.16 & 66.14 & 65.90 & 65.56 & 64.77 & 62.67 \\
\hline
\end{tabular}


Table B - 13. Temperature $\left({ }^{\circ} \mathrm{C}\right)$ map of the east side of plate $1 \mathrm{~T} 2$ at EOC $142 \mathrm{~B}(52.0 \mathrm{EFPD})$.

\begin{tabular}{|c|c|c|c|c|c|c|c|c|c|c|}
\hline \multirow{2}{*}{$\begin{array}{c}\text { Length of } \\
\text { Plate (in) }\end{array}$} & \multicolumn{10}{|c|}{ Width of Plate (inches) } \\
\hline & 0.05 & 0.25 & 0.50 & 0.75 & 1.00 & 1.25 & 1.50 & 1.75 & 2.00 & 2.24 \\
\hline 0.125 & 52.10 & 51.80 & 51.77 & 51.77 & 51.77 & 51.77 & 51.77 & 51.78 & 51.86 & 52.14 \\
\hline 1.00 & 53.51 & 52.46 & 52.57 & 52.58 & 52.59 & 52.59 & 52.58 & 52.52 & 52.47 & 53.36 \\
\hline 2.00 & 57.97 & 78.86 & 81.31 & 81.36 & 81.37 & 81.37 & 81.36 & 81.21 & 68.95 & 56.13 \\
\hline 3.00 & 58.44 & 79.14 & 81.62 & 81.67 & 81.68 & 81.68 & 81.67 & 81.50 & 69.26 & 56.43 \\
\hline 4.00 & 59.63 & 84.80 & 87.69 & 87.75 & 87.76 & 87.76 & 87.75 & 87.57 & 73.07 & 57.33 \\
\hline 5.00 & 60.00 & 85.04 & 87.93 & 88.00 & 88.01 & 88.01 & 87.99 & 87.81 & 73.32 & 57.58 \\
\hline 6.00 & 61.19 & 90.43 & 93.70 & 93.78 & 93.79 & 93.79 & 93.77 & 93.57 & 76.99 & 58.35 \\
\hline 7.00 & 61.54 & 90.68 & 93.95 & 94.03 & 94.05 & 94.04 & 94.02 & 93.81 & 77.26 & 58.61 \\
\hline 8.00 & 62.56 & 94.80 & 98.34 & 98.43 & 98.45 & 98.44 & 98.42 & 98.20 & 80.14 & 59.28 \\
\hline 9.00 & 62.90 & 95.05 & 98.60 & 98.70 & 98.72 & 98.71 & 98.68 & 98.45 & 80.60 & 60.52 \\
\hline 10.00 & 63.74 & 98.36 & 102.38 & 102.49 & 102.52 & 102.51 & 102.47 & 102.20 & 82.86 & 60.88 \\
\hline 11.00 & 64.08 & 98.66 & 102.69 & 102.81 & 102.84 & 102.83 & 102.78 & 102.50 & 83.06 & 60.69 \\
\hline 12.00 & 64.75 & 100.82 & 105.00 & 105.13 & 105.16 & 105.16 & 105.10 & 104.80 & 84.54 & 60.98 \\
\hline 13.00 & 65.08 & 101.13 & 105.31 & 105.45 & 105.48 & 105.48 & 105.41 & 105.11 & 84.85 & 61.34 \\
\hline 14.00 & 65.62 & 103.01 & 107.32 & 107.46 & 107.50 & 107.50 & 107.43 & 107.11 & 86.14 & 61.83 \\
\hline 15.00 & 65.95 & 103.32 & 107.64 & 107.79 & 107.82 & 107.82 & 107.75 & 107.42 & 86.42 & 62.02 \\
\hline 16.00 & 66.39 & 104.27 & 108.64 & 108.79 & 108.83 & 108.83 & 108.75 & 108.41 & 87.12 & 62.24 \\
\hline 17.00 & 66.69 & 104.58 & 108.95 & 109.11 & 109.16 & 109.15 & 109.06 & 108.72 & 87.53 & 63.37 \\
\hline 18.00 & 67.11 & 105.77 & 110.21 & 110.38 & 110.43 & 110.42 & 110.33 & 109.98 & 88.50 & 64.05 \\
\hline 19.00 & 67.42 & 106.09 & 110.53 & 110.71 & 110.75 & 110.75 & 110.65 & 110.29 & 88.68 & 64.10 \\
\hline 20.00 & 67.84 & 107.22 & 111.73 & 111.91 & 111.96 & 111.96 & 111.85 & 111.48 & 89.39 & 63.76 \\
\hline 21.00 & 68.09 & 107.50 & 112.01 & 112.20 & 112.25 & 112.25 & 112.14 & 111.76 & 89.64 & 63.73 \\
\hline 22.00 & 62.00 & 64.89 & 66.21 & 66.38 & 66.44 & 66.43 & 66.30 & 65.86 & 62.76 & 60.06 \\
\hline 22.50 & 60.00 & 59.28 & 59.46 & 59.60 & 59.66 & 59.65 & 59.52 & 59.30 & 59.27 & 59.62 \\
\hline
\end{tabular}


Table B - 14. Temperature $\left({ }^{\circ} \mathrm{C}\right)$ map of the west side of plate 1T2 at EOC 142B (52.0 EFPD).

\begin{tabular}{|c||c|c|c|c|c|c|c|c|c|c|}
\hline \multirow{2}{*}{$\begin{array}{c}\text { Length of } \\
\text { Plate (in) }\end{array}$} & \multicolumn{10}{|c|}{ Width of Plate (inches) } \\
\hline & 0.05 & 0.25 & 0.50 & 0.75 & 1.00 & 1.25 & 1.50 & 1.75 & 2.00 & 2.24 \\
\hline 0.125 & 52.36 & 51.80 & 51.77 & 51.77 & 51.77 & 51.77 & 51.77 & 51.78 & 51.85 & 52.34 \\
\hline 1.00 & 53.87 & 52.46 & 52.57 & 52.58 & 52.58 & 52.58 & 52.58 & 52.52 & 52.47 & 53.62 \\
\hline 2.00 & 57.76 & 78.83 & 81.29 & 81.34 & 81.35 & 81.35 & 81.34 & 81.19 & 68.93 & 55.66 \\
\hline 3.00 & 58.27 & 79.09 & 81.56 & 81.61 & 81.62 & 81.62 & 81.61 & 81.45 & 69.22 & 55.77 \\
\hline 4.00 & 59.39 & 84.71 & 87.59 & 87.66 & 87.66 & 87.66 & 87.65 & 87.48 & 72.99 & 56.60 \\
\hline 5.00 & 59.79 & 84.90 & 87.79 & 87.86 & 87.86 & 87.86 & 87.85 & 87.67 & 73.20 & 56.77 \\
\hline 6.00 & 60.93 & 90.25 & 93.52 & 93.59 & 93.60 & 93.59 & 93.58 & 93.38 & 76.83 & 57.45 \\
\hline 7.00 & 61.35 & 90.48 & 93.74 & 93.82 & 93.83 & 93.83 & 93.81 & 93.60 & 77.07 & 57.76 \\
\hline 8.00 & 62.31 & 94.51 & 98.05 & 98.13 & 98.14 & 98.14 & 98.12 & 97.90 & 79.87 & 58.35 \\
\hline 9.00 & 62.68 & 94.71 & 98.25 & 98.33 & 98.34 & 98.34 & 98.32 & 98.10 & 80.28 & 60.57 \\
\hline 10.00 & 63.51 & 97.98 & 101.91 & 102.02 & 102.03 & 102.03 & 102.00 & 101.73 & 82.50 & 60.65 \\
\hline 11.00 & 63.87 & 98.19 & 102.16 & 102.27 & 102.28 & 102.28 & 102.24 & 101.97 & 82.64 & 59.90 \\
\hline 12.00 & 64.53 & 100.24 & 104.40 & 104.51 & 104.54 & 104.53 & 104.49 & 104.20 & 84.07 & 59.78 \\
\hline 13.00 & 64.87 & 100.48 & 104.65 & 104.77 & 104.79 & 104.79 & 104.74 & 104.44 & 84.34 & 60.08 \\
\hline 14.00 & 65.43 & 102.30 & 106.60 & 106.72 & 106.75 & 106.74 & 106.69 & 106.38 & 85.58 & 60.66 \\
\hline 15.00 & 65.76 & 102.55 & 106.85 & 106.98 & 107.01 & 107.00 & 106.94 & 106.63 & 85.80 & 60.91 \\
\hline 16.00 & 66.19 & 103.44 & 107.78 & 107.91 & 107.94 & 107.94 & 107.88 & 107.55 & 86.45 & 61.10 \\
\hline 17.00 & 66.50 & 103.68 & 108.03 & 108.17 & 108.20 & 108.19 & 108.13 & 107.80 & 86.80 & 63.14 \\
\hline 18.00 & 66.91 & 104.82 & 109.23 & 109.38 & 109.41 & 109.40 & 109.33 & 108.99 & 87.72 & 64.12 \\
\hline 19.00 & 67.22 & 105.07 & 109.49 & 109.64 & 109.67 & 109.67 & 109.59 & 109.24 & 87.86 & 64.00 \\
\hline 20.00 & 67.63 & 106.14 & 110.62 & 110.77 & 110.81 & 110.80 & 110.72 & 110.37 & 88.52 & 62.82 \\
\hline 21.00 & 67.85 & 106.37 & 110.84 & 111.00 & 111.04 & 111.03 & 110.95 & 110.59 & 88.72 & 62.37 \\
\hline 22.00 & 62.42 & 64.01 & 65.30 & 65.44 & 65.49 & 65.49 & 65.37 & 64.95 & 61.86 & 59.36 \\
\hline 22.50 & 60.75 & 58.36 & 58.51 & 58.62 & 58.68 & 58.67 & 58.55 & 58.35 & 58.32 & 60.58 \\
\hline
\end{tabular}


Table B - 15. Temperature $\left({ }^{\circ} \mathrm{C}\right)$ map of the east side of plate 1B5 at EOC 142B (52.0 EFPD).

\begin{tabular}{|c||c|c|c|c|c|c|c|c|c|c|}
\hline \multirow{2}{*}{$\begin{array}{l}\text { Length of } \\
\text { Plate (in) }\end{array}$} & \multicolumn{10}{|c|}{ Width of Plate (inches) } \\
\hline 22.625 & 60.69 & 58.43 & 58.58 & 58.70 & 58.75 & 58.74 & 58.62 & 58.42 & 58.34 & 59.93 \\
\hline 23.00 & 62.52 & 64.09 & 65.36 & 65.51 & 65.56 & 65.55 & 65.43 & 65.02 & 61.95 & 59.45 \\
\hline 24.00 & 68.24 & 106.83 & 111.29 & 111.45 & 111.50 & 111.49 & 111.39 & 111.04 & 89.15 & 62.69 \\
\hline 25.00 & 68.50 & 107.13 & 111.59 & 111.76 & 111.80 & 111.80 & 111.69 & 111.33 & 89.43 & 63.54 \\
\hline 26.00 & 68.64 & 106.82 & 111.25 & 111.42 & 111.47 & 111.46 & 111.35 & 110.98 & 89.43 & 65.27 \\
\hline 27.00 & 68.82 & 107.07 & 111.50 & 111.68 & 111.73 & 111.72 & 111.61 & 111.23 & 89.78 & 65.89 \\
\hline 28.00 & 69.00 & 107.33 & 111.77 & 111.94 & 111.99 & 111.98 & 111.87 & 111.49 & 89.90 & 65.35 \\
\hline 29.00 & 69.18 & 107.59 & 112.03 & 112.21 & 112.26 & 112.25 & 112.13 & 111.75 & 90.03 & 64.20 \\
\hline 30.00 & 69.24 & 107.22 & 111.62 & 111.80 & 111.85 & 111.84 & 111.72 & 111.33 & 89.86 & 63.78 \\
\hline 31.00 & 69.36 & 107.45 & 111.85 & 112.03 & 112.09 & 112.07 & 111.95 & 111.55 & 90.09 & 63.91 \\
\hline 32.00 & 69.24 & 106.12 & 110.40 & 110.59 & 110.64 & 110.63 & 110.50 & 110.10 & 89.31 & 63.65 \\
\hline 33.00 & 69.35 & 106.36 & 110.64 & 110.83 & 110.89 & 110.88 & 110.74 & 110.34 & 89.50 & 63.81 \\
\hline 34.00 & 69.22 & 105.01 & 109.17 & 109.37 & 109.42 & 109.41 & 109.27 & 108.86 & 88.72 & 64.40 \\
\hline 35.00 & 69.29 & 105.21 & 109.38 & 109.58 & 109.64 & 109.62 & 109.48 & 109.07 & 89.01 & 65.79 \\
\hline 36.00 & 69.03 & 103.26 & 107.26 & 107.46 & 107.52 & 107.51 & 107.36 & 106.95 & 87.96 & 66.43 \\
\hline 37.00 & 69.07 & 103.46 & 107.46 & 107.66 & 107.72 & 107.71 & 107.55 & 107.14 & 88.01 & 65.78 \\
\hline 38.00 & 68.67 & 100.71 & 104.48 & 104.68 & 104.75 & 104.73 & 104.57 & 104.16 & 86.24 & 64.23 \\
\hline 39.00 & 68.67 & 100.88 & 104.66 & 104.86 & 104.92 & 104.91 & 104.75 & 104.34 & 86.38 & 63.71 \\
\hline 40.00 & 68.08 & 96.92 & 100.36 & 100.57 & 100.63 & 100.61 & 100.45 & 100.04 & 83.95 & 63.43 \\
\hline 41.00 & 68.02 & 97.06 & 100.50 & 100.71 & 100.77 & 100.75 & 100.59 & 100.18 & 84.08 & 63.63 \\
\hline 42.00 & 67.29 & 92.58 & 95.63 & 95.83 & 95.90 & 95.88 & 95.71 & 95.31 & 81.26 & 62.93 \\
\hline 43.00 & 67.18 & 92.69 & 95.74 & 95.94 & 96.01 & 95.99 & 95.82 & 95.42 & 81.36 & 63.42 \\
\hline 44.00 & 63.22 & 64.39 & 64.93 & 65.13 & 65.21 & 65.19 & 64.99 & 64.62 & 63.66 & 61.93 \\
\hline 45.00 & 62.47 & 63.81 & 64.17 & 64.36 & 64.44 & 64.41 & 64.21 & 63.92 & 63.27 & 61.71 \\
\hline
\end{tabular}


Table B - 16. Temperature $\left({ }^{\circ} \mathrm{C}\right)$ map of the west side of plate 1B5 at EOC 142B (52.0 EFPD).

\begin{tabular}{|c||c|c|c|r|r|r|r|r|r|r|}
\hline \multirow{2}{*}{$\begin{array}{c}\text { Length of } \\
\text { Plate (in) }\end{array}$} & \multicolumn{10}{|c|}{ Width of Plate (inches) } \\
\hline 22.625 & 60.10 & 59.34 & 59.53 & 59.67 & 59.74 & 59.73 & 59.59 & 59.37 & 59.29 & 59.44 \\
\hline 23.00 & 62.04 & 65.00 & 66.30 & 66.47 & 66.53 & 66.52 & 66.38 & 65.96 & 62.87 & 60.11 \\
\hline 24.00 & 68.29 & 108.08 & 112.56 & 112.76 & 112.81 & 112.81 & 112.69 & 112.32 & 90.15 & 63.94 \\
\hline 25.00 & 68.56 & 108.41 & 112.90 & 113.10 & 113.16 & 113.16 & 113.03 & 112.65 & 90.47 & 64.41 \\
\hline 26.00 & 68.73 & 108.14 & 112.60 & 112.80 & 112.86 & 112.86 & 112.73 & 112.35 & 90.51 & 65.31 \\
\hline 27.00 & 68.95 & 108.43 & 112.90 & 113.10 & 113.17 & 113.16 & 113.03 & 112.64 & 90.90 & 65.75 \\
\hline 28.00 & 69.16 & 108.73 & 113.20 & 113.41 & 113.47 & 113.47 & 113.33 & 112.94 & 91.04 & 65.57 \\
\hline 29.00 & 69.36 & 109.04 & 113.52 & 113.73 & 113.80 & 113.79 & 113.65 & 113.25 & 91.22 & 65.16 \\
\hline 30.00 & 69.46 & 108.71 & 113.15 & 113.36 & 113.43 & 113.43 & 113.28 & 112.88 & 91.09 & 65.07 \\
\hline 31.00 & 69.62 & 108.98 & 113.42 & 113.64 & 113.71 & 113.70 & 113.56 & 113.15 & 91.38 & 65.29 \\
\hline 32.00 & 69.54 & 107.70 & 112.03 & 112.25 & 112.32 & 112.31 & 112.16 & 111.75 & 90.63 & 65.18 \\
\hline 33.00 & 69.69 & 107.96 & 112.29 & 112.51 & 112.59 & 112.58 & 112.42 & 112.01 & 90.86 & 65.22 \\
\hline 34.00 & 69.61 & 106.70 & 110.90 & 111.13 & 111.21 & 111.20 & 111.04 & 110.62 & 90.13 & 65.46 \\
\hline 35.00 & 69.73 & 106.95 & 111.16 & 111.39 & 111.47 & 111.46 & 111.30 & 110.87 & 90.49 & 66.17 \\
\hline 36.00 & 69.52 & 105.06 & 109.10 & 109.33 & 109.41 & 109.40 & 109.24 & 108.81 & 89.47 & 66.45 \\
\hline 37.00 & 69.61 & 105.29 & 109.34 & 109.58 & 109.66 & 109.65 & 109.48 & 109.05 & 89.56 & 66.16 \\
\hline 38.00 & 69.26 & 102.62 & 106.44 & 106.68 & 106.76 & 106.75 & 106.57 & 106.15 & 87.82 & 65.35 \\
\hline 39.00 & 69.32 & 102.84 & 106.66 & 106.90 & 106.98 & 106.97 & 106.79 & 106.37 & 88.00 & 65.19 \\
\hline 40.00 & 68.75 & 98.95 & 102.44 & 102.68 & 102.77 & 102.76 & 102.57 & 102.15 & 85.63 & 64.94 \\
\hline 41.00 & 68.76 & 99.14 & 102.63 & 102.87 & 102.96 & 102.95 & 102.76 & 102.34 & 85.80 & 65.04 \\
\hline 42.00 & 68.06 & 94.74 & 97.84 & 98.08 & 98.17 & 98.16 & 97.97 & 97.55 & 83.04 & 64.40 \\
\hline 43.00 & 68.02 & 94.89 & 97.99 & 98.23 & 98.32 & 98.31 & 98.12 & 97.71 & 83.18 & 64.58 \\
\hline 44.00 & 63.63 & 66.04 & 66.62 & 66.85 & 66.95 & 66.93 & 66.71 & 66.33 & 65.34 & 62.21 \\
\hline 45.00 & 62.83 & 65.46 & 65.87 & 66.09 & 66.18 & 66.17 & 65.95 & 65.64 & 64.97 & 61.81 \\
\hline
\end{tabular}


Table B - 17. Temperature $\left({ }^{\circ} \mathrm{C}\right)$ map of the east side of plate 1T2 at BOC 143A (52.0 EFPD cumulative).

\begin{tabular}{|c||c|c|c|c|c|c|c|c|c|c|}
\hline \multirow{2}{*}{$\begin{array}{c}\text { Length of } \\
\text { Plate (in) }\end{array}$} & \multicolumn{10}{|c|}{ Width of Plate (inches) } \\
\hline & 0.05 & 0.25 & 0.50 & 0.75 & 1.00 & 1.25 & 1.50 & 1.75 & 2.00 & 2.24 \\
\hline 0.125 & 52.08 & 51.79 & 51.77 & 51.77 & 51.77 & 51.77 & 51.77 & 51.77 & 51.85 & 52.12 \\
\hline 1.00 & 53.11 & 52.31 & 52.39 & 52.40 & 52.40 & 52.40 & 52.40 & 52.35 & 52.32 & 53.02 \\
\hline 2.00 & 55.88 & 73.28 & 75.32 & 75.36 & 75.37 & 75.37 & 75.36 & 75.23 & 65.20 & 54.60 \\
\hline 3.00 & 56.23 & 73.48 & 75.54 & 75.58 & 75.58 & 75.58 & 75.58 & 75.43 & 65.42 & 54.81 \\
\hline 4.00 & 57.09 & 78.29 & 80.73 & 80.78 & 80.79 & 80.79 & 80.78 & 80.62 & 68.58 & 55.46 \\
\hline 5.00 & 57.36 & 78.44 & 80.88 & 80.93 & 80.93 & 80.93 & 80.93 & 80.76 & 68.74 & 55.64 \\
\hline 6.00 & 58.04 & 81.70 & 84.38 & 84.44 & 84.45 & 84.44 & 84.43 & 84.26 & 70.91 & 56.07 \\
\hline 7.00 & 58.33 & 81.86 & 84.54 & 84.60 & 84.61 & 84.61 & 84.60 & 84.42 & 71.08 & 56.27 \\
\hline 8.00 & 59.05 & 85.16 & 88.08 & 88.14 & 88.15 & 88.15 & 88.14 & 87.95 & 73.32 & 56.75 \\
\hline 9.00 & 59.30 & 85.30 & 88.22 & 88.29 & 88.30 & 88.30 & 88.28 & 88.09 & 73.64 & 57.75 \\
\hline 10.00 & 59.99 & 88.38 & 91.52 & 91.59 & 91.60 & 91.60 & 91.58 & 91.38 & 75.68 & 58.05 \\
\hline 11.00 & 60.24 & 88.54 & 91.67 & 91.75 & 91.76 & 91.75 & 91.73 & 91.53 & 75.77 & 57.87 \\
\hline 12.00 & 60.82 & 90.71 & 93.99 & 94.07 & 94.08 & 94.08 & 94.06 & 93.85 & 77.30 & 58.12 \\
\hline 13.00 & 61.06 & 90.87 & 94.15 & 94.23 & 94.24 & 94.24 & 94.21 & 94.00 & 77.49 & 58.40 \\
\hline 14.00 & 61.55 & 92.75 & 96.15 & 96.23 & 96.24 & 96.24 & 96.22 & 96.00 & 78.83 & 58.82 \\
\hline 15.00 & 61.79 & 92.92 & 96.31 & 96.39 & 96.41 & 96.41 & 96.38 & 96.16 & 78.99 & 58.96 \\
\hline 16.00 & 62.26 & 94.47 & 97.96 & 98.05 & 98.07 & 98.06 & 98.03 & 97.81 & 80.11 & 59.20 \\
\hline 17.00 & 62.48 & 94.64 & 98.13 & 98.22 & 98.24 & 98.24 & 98.20 & 97.97 & 80.40 & 60.14 \\
\hline 18.00 & 62.87 & 96.00 & 99.67 & 99.78 & 99.80 & 99.79 & 99.75 & 99.49 & 81.49 & 60.73 \\
\hline 19.00 & 63.10 & 96.17 & 99.87 & 99.98 & 100.00 & 100.00 & 99.95 & 99.68 & 81.57 & 60.75 \\
\hline 20.00 & 63.36 & 96.65 & 100.42 & 100.54 & 100.56 & 100.56 & 100.51 & 100.23 & 81.88 & 60.40 \\
\hline 21.00 & 63.53 & 96.80 & 100.59 & 100.70 & 100.73 & 100.73 & 100.67 & 100.39 & 82.03 & 60.35 \\
\hline 22.00 & 59.20 & 61.08 & 62.12 & 62.22 & 62.25 & 62.24 & 62.17 & 61.86 & 59.36 & 57.88 \\
\hline 22.50 & 57.86 & 56.31 & 56.35 & 56.42 & 56.45 & 56.44 & 56.38 & 56.26 & 56.44 & 57.82 \\
\hline
\end{tabular}


Table B - 18. Temperature $\left({ }^{\circ} \mathrm{C}\right)$ map of the west side of plate 1T2 at BOC 143A (52.0 EFPD cumulative).

\begin{tabular}{|c|c|c|c|c|c|c|c|c|c|c|}
\hline \multirow{2}{*}{$\begin{array}{c}\text { Length of } \\
\text { Plate (in) }\end{array}$} & \multicolumn{10}{|c|}{ Width of Plate (inches) } \\
\hline & 0.05 & 0.25 & 0.50 & 0.75 & 1.00 & 1.25 & 1.50 & 1.75 & 2.00 & 2.24 \\
\hline 0.125 & 52.33 & 51.79 & 51.77 & 51.77 & 51.77 & 51.77 & 51.77 & 51.77 & 51.85 & 52.31 \\
\hline 1.00 & 53.58 & 52.31 & 52.39 & 52.40 & 52.40 & 52.40 & 52.40 & 52.35 & 52.32 & 53.35 \\
\hline 2.00 & 56.38 & 73.29 & 75.33 & 75.37 & 75.37 & 75.37 & 75.37 & 75.24 & 65.20 & 54.62 \\
\hline 3.00 & 56.79 & 73.49 & 75.55 & 75.59 & 75.60 & 75.59 & 75.59 & 75.44 & 65.42 & 54.69 \\
\hline 4.00 & 57.69 & 78.31 & 80.75 & 80.80 & 80.80 & 80.80 & 80.80 & 80.64 & 68.58 & 55.34 \\
\hline 5.00 & 58.01 & 78.46 & 80.90 & 80.95 & 80.96 & 80.96 & 80.95 & 80.78 & 68.74 & 55.46 \\
\hline 6.00 & 58.74 & 81.73 & 84.41 & 84.47 & 84.47 & 84.47 & 84.46 & 84.29 & 70.92 & 55.89 \\
\hline 7.00 & 59.08 & 81.90 & 84.58 & 84.64 & 84.65 & 84.65 & 84.63 & 84.45 & 71.10 & 56.13 \\
\hline 8.00 & 59.83 & 85.20 & 88.12 & 88.19 & 88.20 & 88.20 & 88.18 & 87.99 & 73.33 & 56.58 \\
\hline 9.00 & 60.12 & 85.35 & 88.28 & 88.35 & 88.35 & 88.35 & 88.33 & 88.14 & 73.66 & 58.41 \\
\hline 10.00 & 60.85 & 88.44 & 91.58 & 91.65 & 91.66 & 91.66 & 91.64 & 91.44 & 75.71 & 58.50 \\
\hline 11.00 & 61.14 & 88.60 & 91.74 & 91.82 & 91.83 & 91.83 & 91.80 & 91.59 & 75.81 & 57.88 \\
\hline 12.00 & 61.75 & 90.79 & 94.07 & 94.15 & 94.16 & 94.16 & 94.13 & 93.92 & 77.34 & 57.80 \\
\hline 13.00 & 62.03 & 90.96 & 94.24 & 94.32 & 94.33 & 94.33 & 94.30 & 94.08 & 77.54 & 58.04 \\
\hline 14.00 & 62.55 & 92.85 & 96.24 & 96.33 & 96.35 & 96.35 & 96.31 & 96.09 & 78.88 & 58.55 \\
\hline 15.00 & 62.82 & 93.02 & 96.42 & 96.51 & 96.52 & 96.52 & 96.49 & 96.25 & 79.05 & 58.74 \\
\hline 16.00 & 63.31 & 94.59 & 98.08 & 98.18 & 98.20 & 98.19 & 98.15 & 97.91 & 80.18 & 58.97 \\
\hline 17.00 & 63.56 & 94.77 & 98.27 & 98.38 & 98.40 & 98.40 & 98.35 & 98.08 & 80.47 & 60.68 \\
\hline 18.00 & 63.96 & 96.13 & 99.83 & 99.95 & 99.97 & 99.97 & 99.91 & 99.63 & 81.56 & 61.54 \\
\hline 19.00 & 64.21 & 96.32 & 100.04 & 100.16 & 100.19 & 100.18 & 100.13 & 99.84 & 81.66 & 61.43 \\
\hline 20.00 & 64.49 & 96.81 & 100.61 & 100.74 & 100.76 & 100.76 & 100.70 & 100.40 & 81.98 & 60.39 \\
\hline 21.00 & 64.66 & 96.97 & 100.79 & 100.92 & 100.94 & 100.94 & 100.87 & 100.57 & 82.13 & 59.99 \\
\hline 22.00 & 60.40 & 61.23 & 62.28 & 62.38 & 62.41 & 62.41 & 62.33 & 62.00 & 59.48 & 57.77 \\
\hline 22.50 & 59.13 & 56.48 & 56.52 & 56.59 & 56.62 & 56.62 & 56.54 & 56.41 & 56.55 & 59.01 \\
\hline
\end{tabular}


Table B - 19. Temperature $\left({ }^{\circ} \mathrm{C}\right)$ map of the east side of plate 1B5 at BOC 143A (52.0 EFPD cumulative).

\begin{tabular}{|c||c|c|r|r|r|r|r|r|r|c|}
\hline \multirow{2}{*}{$\begin{array}{l}\text { Length of } \\
\text { Plate (in) }\end{array}$} & \multicolumn{10}{|c|}{ Width of Plate (inches) } \\
\hline 22.625 & 57.91 & 56.36 & 56.41 & 56.48 & 56.51 & 56.50 & 56.43 & 56.31 & 56.45 & 57.65 \\
\hline 23.00 & 59.31 & 61.12 & 62.14 & 62.24 & 62.27 & 62.27 & 62.20 & 61.89 & 59.42 & 57.99 \\
\hline 24.00 & 63.92 & 97.10 & 100.89 & 101.01 & 101.04 & 101.04 & 100.98 & 100.71 & 82.35 & 60.72 \\
\hline 25.00 & 64.12 & 97.36 & 101.17 & 101.30 & 101.33 & 101.32 & 101.26 & 100.98 & 82.60 & 61.09 \\
\hline 26.00 & 64.27 & 97.55 & 101.37 & 101.50 & 101.53 & 101.52 & 101.46 & 101.17 & 82.88 & 61.85 \\
\hline 27.00 & 64.39 & 97.73 & 101.56 & 101.69 & 101.72 & 101.71 & 101.65 & 101.36 & 83.16 & 62.18 \\
\hline 28.00 & 64.38 & 96.91 & 100.66 & 100.79 & 100.82 & 100.82 & 100.75 & 100.46 & 82.58 & 61.91 \\
\hline 29.00 & 64.49 & 97.10 & 100.85 & 100.98 & 101.01 & 101.01 & 100.94 & 100.64 & 82.65 & 61.51 \\
\hline 30.00 & 64.44 & 96.06 & 99.71 & 99.85 & 99.88 & 99.87 & 99.80 & 99.51 & 82.03 & 61.34 \\
\hline 31.00 & 64.52 & 96.22 & 99.87 & 100.01 & 100.04 & 100.04 & 99.96 & 99.67 & 82.20 & 61.49 \\
\hline 32.00 & 64.40 & 94.98 & 98.51 & 98.64 & 98.68 & 98.67 & 98.59 & 98.30 & 81.44 & 61.37 \\
\hline 33.00 & 64.47 & 95.13 & 98.68 & 98.82 & 98.85 & 98.85 & 98.77 & 98.47 & 81.56 & 61.36 \\
\hline 34.00 & 64.30 & 93.79 & 97.16 & 97.30 & 97.34 & 97.33 & 97.25 & 96.95 & 80.73 & 61.49 \\
\hline 35.00 & 64.35 & 93.92 & 97.31 & 97.45 & 97.49 & 97.48 & 97.40 & 97.10 & 80.95 & 62.02 \\
\hline 36.00 & 64.03 & 91.73 & 94.82 & 94.96 & 95.00 & 94.99 & 94.91 & 94.62 & 79.64 & 62.14 \\
\hline 37.00 & 64.04 & 91.85 & 94.96 & 95.09 & 95.13 & 95.13 & 95.04 & 94.75 & 79.64 & 61.86 \\
\hline 38.00 & 63.66 & 89.36 & 92.23 & 92.35 & 92.39 & 92.38 & 92.30 & 92.04 & 77.95 & 61.15 \\
\hline 39.00 & 63.65 & 89.46 & 92.32 & 92.45 & 92.49 & 92.48 & 92.40 & 92.14 & 78.04 & 60.97 \\
\hline 40.00 & 63.20 & 86.57 & 89.21 & 89.34 & 89.38 & 89.37 & 89.29 & 89.03 & 76.19 & 60.77 \\
\hline 41.00 & 63.15 & 86.65 & 89.29 & 89.42 & 89.46 & 89.45 & 89.37 & 89.11 & 76.27 & 60.80 \\
\hline 42.00 & 62.48 & 82.48 & 84.79 & 84.91 & 84.95 & 84.95 & 84.86 & 84.60 & 73.55 & 60.22 \\
\hline 43.00 & 62.40 & 82.53 & 84.84 & 84.97 & 85.01 & 85.00 & 84.91 & 84.66 & 73.61 & 60.32 \\
\hline 44.00 & 59.54 & 60.34 & 60.69 & 60.82 & 60.86 & 60.85 & 60.73 & 60.50 & 59.92 & 58.90 \\
\hline 45.00 & 59.02 & 59.87 & 60.07 & 60.18 & 60.23 & 60.21 & 60.10 & 59.93 & 59.62 & 58.68 \\
\hline
\end{tabular}


Table B - 20. Temperature $\left({ }^{\circ} \mathrm{C}\right)$ map of the west side of plate 1B5 at BOC 143A (52.0 EFPD cumulative).

\begin{tabular}{|c||c|c|r|r|r|r|r|r|r|r|}
\hline \multirow{2}{*}{$\begin{array}{c}\text { Length of } \\
\text { Plate (in) }\end{array}$} & \multicolumn{10}{|c|}{ Width of Plate (inches) } \\
\hline 22.625 & 59.08 & 56.52 & 56.58 & 56.65 & 56.68 & 56.68 & 56.60 & 56.46 & 56.56 & 58.44 \\
\hline 23.00 & 60.51 & 61.29 & 62.31 & 62.42 & 62.45 & 62.44 & 62.36 & 62.04 & 59.55 & 57.88 \\
\hline 24.00 & 65.06 & 97.33 & 101.14 & 101.27 & 101.30 & 101.29 & 101.22 & 100.93 & 82.49 & 60.36 \\
\hline 25.00 & 65.27 & 97.61 & 101.43 & 101.57 & 101.60 & 101.59 & 101.52 & 101.21 & 82.74 & 61.07 \\
\hline 26.00 & 65.42 & 97.82 & 101.64 & 101.78 & 101.82 & 101.81 & 101.73 & 101.42 & 83.03 & 62.54 \\
\hline 27.00 & 65.53 & 98.01 & 101.85 & 101.99 & 102.02 & 102.01 & 101.93 & 101.61 & 83.32 & 63.02 \\
\hline 28.00 & 65.52 & 97.20 & 100.96 & 101.10 & 101.14 & 101.13 & 101.04 & 100.72 & 82.75 & 62.46 \\
\hline 29.00 & 65.62 & 97.41 & 101.16 & 101.31 & 101.35 & 101.34 & 101.25 & 100.92 & 82.83 & 61.47 \\
\hline 30.00 & 65.55 & 96.38 & 100.04 & 100.19 & 100.23 & 100.22 & 100.12 & 99.80 & 82.22 & 61.04 \\
\hline 31.00 & 65.61 & 96.55 & 100.22 & 100.37 & 100.40 & 100.40 & 100.30 & 99.97 & 82.39 & 61.12 \\
\hline 32.00 & 65.47 & 95.31 & 98.87 & 99.01 & 99.05 & 99.05 & 98.94 & 98.62 & 81.65 & 60.88 \\
\hline 33.00 & 65.52 & 95.49 & 99.05 & 99.20 & 99.24 & 99.23 & 99.13 & 98.80 & 81.78 & 60.98 \\
\hline 34.00 & 65.34 & 94.12 & 97.55 & 97.70 & 97.74 & 97.73 & 97.63 & 97.30 & 80.96 & 61.42 \\
\hline 35.00 & 65.35 & 94.27 & 97.71 & 97.86 & 97.90 & 97.89 & 97.78 & 97.45 & 81.18 & 62.50 \\
\hline 36.00 & 65.00 & 92.08 & 95.24 & 95.39 & 95.43 & 95.42 & 95.31 & 94.98 & 79.88 & 62.92 \\
\hline 37.00 & 64.98 & 92.21 & 95.38 & 95.53 & 95.58 & 95.56 & 95.45 & 95.12 & 79.89 & 62.35 \\
\hline 38.00 & 64.57 & 89.74 & 92.62 & 92.76 & 92.80 & 92.79 & 92.68 & 92.39 & 78.21 & 61.06 \\
\hline 39.00 & 64.52 & 89.84 & 92.73 & 92.87 & 92.91 & 92.90 & 92.79 & 92.49 & 78.30 & 60.61 \\
\hline 40.00 & 64.04 & 86.97 & 89.63 & 89.77 & 89.81 & 89.80 & 89.69 & 89.39 & 76.46 & 60.38 \\
\hline 41.00 & 63.95 & 87.06 & 89.72 & 89.86 & 89.90 & 89.89 & 89.78 & 89.48 & 76.55 & 60.49 \\
\hline 42.00 & 63.24 & 82.90 & 85.23 & 85.37 & 85.42 & 85.40 & 85.28 & 84.99 & 73.84 & 59.87 \\
\hline 43.00 & 63.10 & 82.96 & 85.29 & 85.43 & 85.47 & 85.46 & 85.34 & 85.05 & 73.90 & 60.21 \\
\hline 44.00 & 60.23 & 60.68 & 61.05 & 61.18 & 61.23 & 61.22 & 61.07 & 60.81 & 60.19 & 59.25 \\
\hline 45.00 & 59.68 & 60.21 & 60.42 & 60.54 & 60.59 & 60.58 & 60.44 & 60.24 & 59.89 & 59.11 \\
\hline
\end{tabular}


Table B - 21. Temperature $\left({ }^{\circ} \mathrm{C}\right)$ map of the east side of plate 1T2 at MOC 143A (70.0 EFPD cumulative).

\begin{tabular}{|c||c|c|c|c|c|c|c|c|c|c|}
\hline \multirow{2}{*}{$\begin{array}{c}\text { Length of } \\
\text { Plate (in) }\end{array}$} & \multicolumn{10}{|c|}{ Width of Plate (inches) } \\
\hline & 0.05 & 0.25 & 0.50 & 0.75 & 1.00 & 1.25 & 1.50 & 1.75 & 2.00 & 2.24 \\
\hline 0.125 & 52.08 & 51.79 & 51.77 & 51.77 & 51.77 & 51.77 & 51.77 & 51.77 & 51.85 & 52.12 \\
\hline 1.00 & 53.18 & 52.38 & 52.49 & 52.50 & 52.50 & 52.50 & 52.50 & 52.44 & 52.37 & 53.08 \\
\hline 2.00 & 56.38 & 76.54 & 78.86 & 78.91 & 78.91 & 78.91 & 78.90 & 78.76 & 67.31 & 54.90 \\
\hline 3.00 & 56.73 & 76.76 & 79.09 & 79.14 & 79.14 & 79.14 & 79.13 & 78.98 & 67.54 & 55.11 \\
\hline 4.00 & 57.58 & 81.34 & 84.02 & 84.08 & 84.08 & 84.08 & 84.07 & 83.91 & 70.58 & 55.77 \\
\hline 5.00 & 57.85 & 81.50 & 84.18 & 84.23 & 84.24 & 84.24 & 84.23 & 84.06 & 70.75 & 55.95 \\
\hline 6.00 & 58.56 & 84.80 & 87.72 & 87.78 & 87.79 & 87.79 & 87.78 & 87.60 & 72.97 & 56.39 \\
\hline 7.00 & 58.86 & 84.97 & 87.89 & 87.96 & 87.97 & 87.97 & 87.95 & 87.76 & 73.16 & 56.60 \\
\hline 8.00 & 59.54 & 87.98 & 91.11 & 91.18 & 91.19 & 91.19 & 91.17 & 90.98 & 75.22 & 57.06 \\
\hline 9.00 & 59.80 & 88.13 & 91.26 & 91.33 & 91.34 & 91.34 & 91.32 & 91.13 & 75.56 & 58.10 \\
\hline 10.00 & 60.36 & 90.35 & 93.63 & 93.70 & 93.71 & 93.71 & 93.69 & 93.49 & 77.03 & 58.30 \\
\hline 11.00 & 60.62 & 90.51 & 93.78 & 93.86 & 93.87 & 93.87 & 93.85 & 93.64 & 77.13 & 58.12 \\
\hline 12.00 & 61.22 & 92.75 & 96.17 & 96.25 & 96.27 & 96.26 & 96.24 & 96.03 & 78.71 & 58.38 \\
\hline 13.00 & 61.46 & 92.92 & 96.33 & 96.42 & 96.43 & 96.43 & 96.40 & 96.18 & 78.91 & 58.66 \\
\hline 14.00 & 61.77 & 93.56 & 97.00 & 97.09 & 97.11 & 97.10 & 97.08 & 96.85 & 79.42 & 58.98 \\
\hline 15.00 & 62.01 & 93.72 & 97.16 & 97.25 & 97.27 & 97.27 & 97.23 & 97.01 & 79.58 & 59.11 \\
\hline 16.00 & 62.39 & 94.78 & 98.29 & 98.39 & 98.41 & 98.41 & 98.37 & 98.13 & 80.36 & 59.30 \\
\hline 17.00 & 62.62 & 94.94 & 98.47 & 98.58 & 98.60 & 98.60 & 98.56 & 98.30 & 80.65 & 60.24 \\
\hline 18.00 & 62.88 & 95.48 & 99.09 & 99.20 & 99.22 & 99.22 & 99.17 & 98.91 & 81.19 & 60.77 \\
\hline 19.00 & 63.10 & 95.65 & 99.28 & 99.39 & 99.41 & 99.41 & 99.36 & 99.09 & 81.27 & 60.77 \\
\hline 20.00 & 63.32 & 95.88 & 99.54 & 99.66 & 99.68 & 99.68 & 99.63 & 99.35 & 81.40 & 60.40 \\
\hline 21.00 & 63.49 & 96.02 & 99.70 & 99.82 & 99.84 & 99.84 & 99.79 & 99.51 & 81.54 & 60.33 \\
\hline 22.00 & 59.27 & 61.11 & 62.14 & 62.24 & 62.27 & 62.27 & 62.19 & 61.88 & 59.43 & 57.93 \\
\hline 22.50 & 57.96 & 56.47 & 56.52 & 56.59 & 56.62 & 56.62 & 56.55 & 56.43 & 56.59 & 57.90 \\
\hline
\end{tabular}


Table B - 22. Temperature $\left({ }^{\circ} \mathrm{C}\right)$ map of the west side of plate 1T2 at MOC 143A (70.0 EFPD cumulative).

\begin{tabular}{|c||c|c|c|c|c|c|c|c|c|c|}
\hline \multirow{2}{*}{$\begin{array}{c}\text { Length of } \\
\text { Plate (in) }\end{array}$} & \multicolumn{10}{|c|}{ Width of Plate (inches) } \\
\hline & 0.05 & 0.25 & 0.50 & 0.75 & 1.00 & 1.25 & 1.50 & 1.75 & 2.00 & 2.24 \\
\hline 0.125 & 52.33 & 51.79 & 51.77 & 51.77 & 51.77 & 51.77 & 51.77 & 51.77 & 51.85 & 52.31 \\
\hline 1.00 & 53.65 & 52.38 & 52.49 & 52.50 & 52.50 & 52.50 & 52.50 & 52.44 & 52.37 & 53.40 \\
\hline 2.00 & 56.87 & 76.55 & 78.86 & 78.91 & 78.91 & 78.91 & 78.91 & 78.77 & 67.30 & 54.89 \\
\hline 3.00 & 57.29 & 76.77 & 79.10 & 79.15 & 79.15 & 79.15 & 79.14 & 78.99 & 67.54 & 54.96 \\
\hline 4.00 & 58.18 & 81.36 & 84.04 & 84.09 & 84.10 & 84.10 & 84.09 & 83.92 & 70.59 & 55.61 \\
\hline 5.00 & 58.51 & 81.52 & 84.20 & 84.26 & 84.26 & 84.26 & 84.25 & 84.08 & 70.76 & 55.74 \\
\hline 6.00 & 59.25 & 84.83 & 87.75 & 87.81 & 87.82 & 87.82 & 87.81 & 87.62 & 72.98 & 56.18 \\
\hline 7.00 & 59.56 & 84.98 & 87.91 & 87.97 & 87.98 & 87.98 & 87.96 & 87.77 & 73.15 & 56.41 \\
\hline 8.00 & 60.32 & 88.02 & 91.15 & 91.22 & 91.23 & 91.23 & 91.21 & 91.01 & 75.24 & 56.86 \\
\hline 9.00 & 60.62 & 88.18 & 91.31 & 91.39 & 91.40 & 91.40 & 91.38 & 91.17 & 75.57 & 58.75 \\
\hline 10.00 & 61.23 & 90.41 & 93.69 & 93.77 & 93.78 & 93.78 & 93.75 & 93.54 & 77.06 & 58.75 \\
\hline 11.00 & 61.52 & 90.57 & 93.85 & 93.93 & 93.95 & 93.94 & 93.92 & 93.70 & 77.16 & 58.10 \\
\hline 12.00 & 62.15 & 92.83 & 96.25 & 96.34 & 96.35 & 96.35 & 96.32 & 96.09 & 78.75 & 58.02 \\
\hline 13.00 & 62.43 & 93.00 & 96.42 & 96.51 & 96.52 & 96.52 & 96.49 & 96.26 & 78.95 & 58.26 \\
\hline 14.00 & 62.77 & 93.65 & 97.10 & 97.19 & 97.21 & 97.21 & 97.17 & 96.94 & 79.47 & 58.69 \\
\hline 15.00 & 63.04 & 93.82 & 97.27 & 97.36 & 97.38 & 97.38 & 97.34 & 97.10 & 79.63 & 58.87 \\
\hline 16.00 & 63.45 & 94.89 & 98.43 & 98.54 & 98.56 & 98.56 & 98.50 & 98.24 & 80.43 & 59.06 \\
\hline 17.00 & 63.70 & 95.07 & 98.63 & 98.74 & 98.76 & 98.76 & 98.70 & 98.43 & 80.72 & 60.78 \\
\hline 18.00 & 63.98 & 95.62 & 99.25 & 99.37 & 99.40 & 99.39 & 99.33 & 99.05 & 81.26 & 61.58 \\
\hline 19.00 & 64.21 & 95.79 & 99.45 & 99.57 & 99.60 & 99.60 & 99.53 & 99.25 & 81.35 & 61.45 \\
\hline 20.00 & 64.45 & 96.04 & 99.73 & 99.86 & 99.89 & 99.88 & 99.82 & 99.52 & 81.50 & 60.39 \\
\hline 21.00 & 64.62 & 96.19 & 99.90 & 100.03 & 100.06 & 100.06 & 99.99 & 99.69 & 81.65 & 59.98 \\
\hline 22.00 & 60.46 & 61.27 & 62.30 & 62.40 & 62.44 & 62.43 & 62.35 & 62.02 & 59.54 & 57.82 \\
\hline 22.50 & 59.23 & 56.64 & 56.69 & 56.76 & 56.80 & 56.79 & 56.71 & 56.57 & 56.69 & 59.09 \\
\hline
\end{tabular}


Table B - 23. Temperature $\left({ }^{\circ} \mathrm{C}\right)$ map of the east side of plate 1B5 at MOC 143A (70.0 EFPD cumulative).

\begin{tabular}{|c||c|c|r|r|r|r|r|r|r|c|}
\hline \multirow{2}{*}{$\begin{array}{l}\text { Length of } \\
\text { Plate (in) }\end{array}$} & \multicolumn{10}{|c|}{ Width of Plate (inches) } \\
\hline 22.625 & 58.01 & 56.52 & 56.58 & 56.65 & 56.68 & 56.67 & 56.60 & 56.48 & 56.60 & 57.73 \\
\hline 23.00 & 59.37 & 61.14 & 62.15 & 62.25 & 62.28 & 62.27 & 62.20 & 61.89 & 59.48 & 58.04 \\
\hline 24.00 & 63.85 & 96.19 & 99.86 & 99.99 & 100.01 & 100.01 & 99.95 & 99.68 & 81.79 & 60.69 \\
\hline 25.00 & 64.04 & 96.41 & 100.13 & 100.25 & 100.28 & 100.28 & 100.22 & 99.93 & 82.02 & 61.06 \\
\hline 26.00 & 64.18 & 96.51 & 100.23 & 100.36 & 100.39 & 100.39 & 100.32 & 100.04 & 82.25 & 61.80 \\
\hline 27.00 & 64.32 & 96.70 & 100.43 & 100.56 & 100.59 & 100.59 & 100.52 & 100.23 & 82.54 & 62.15 \\
\hline 28.00 & 64.48 & 97.14 & 100.90 & 101.03 & 101.06 & 101.06 & 100.99 & 100.69 & 82.76 & 61.98 \\
\hline 29.00 & 64.60 & 97.33 & 101.09 & 101.23 & 101.26 & 101.26 & 101.18 & 100.89 & 82.84 & 61.59 \\
\hline 30.00 & 64.60 & 96.66 & 100.36 & 100.50 & 100.53 & 100.53 & 100.45 & 100.15 & 82.45 & 61.45 \\
\hline 31.00 & 64.68 & 96.83 & 100.53 & 100.67 & 100.70 & 100.70 & 100.62 & 100.32 & 82.62 & 61.60 \\
\hline 32.00 & 64.62 & 95.97 & 99.59 & 99.73 & 99.77 & 99.76 & 99.68 & 99.38 & 82.12 & 61.51 \\
\hline 33.00 & 64.69 & 96.15 & 99.77 & 99.91 & 99.95 & 99.94 & 99.86 & 99.56 & 82.25 & 61.52 \\
\hline 34.00 & 64.54 & 94.78 & 98.28 & 98.42 & 98.46 & 98.45 & 98.37 & 98.07 & 81.43 & 61.65 \\
\hline 35.00 & 64.58 & 94.93 & 98.43 & 98.58 & 98.61 & 98.61 & 98.52 & 98.22 & 81.66 & 62.19 \\
\hline 36.00 & 64.47 & 93.99 & 97.39 & 97.53 & 97.57 & 97.57 & 97.48 & 97.17 & 81.20 & 62.44 \\
\hline 37.00 & 64.49 & 94.12 & 97.53 & 97.68 & 97.72 & 97.71 & 97.62 & 97.32 & 81.20 & 62.17 \\
\hline 38.00 & 64.12 & 91.70 & 94.80 & 94.94 & 94.98 & 94.98 & 94.88 & 94.58 & 79.56 & 61.45 \\
\hline 39.00 & 64.12 & 91.81 & 94.92 & 95.07 & 95.11 & 95.10 & 95.01 & 94.71 & 79.65 & 61.27 \\
\hline 40.00 & 63.70 & 89.13 & 91.95 & 92.08 & 92.12 & 92.12 & 92.03 & 91.76 & 77.93 & 61.09 \\
\hline 41.00 & 63.66 & 89.21 & 92.04 & 92.18 & 92.22 & 92.21 & 92.12 & 91.85 & 78.02 & 61.14 \\
\hline 42.00 & 63.08 & 85.62 & 88.17 & 88.30 & 88.34 & 88.33 & 88.24 & 87.97 & 75.66 & 60.60 \\
\hline 43.00 & 63.00 & 85.68 & 88.23 & 88.36 & 88.40 & 88.40 & 88.30 & 88.04 & 75.73 & 60.72 \\
\hline 44.00 & 59.77 & 60.69 & 61.08 & 61.21 & 61.26 & 61.25 & 61.13 & 60.88 & 60.23 & 59.08 \\
\hline 45.00 & 59.20 & 60.16 & 60.38 & 60.49 & 60.54 & 60.53 & 60.41 & 60.23 & 59.89 & 58.83 \\
\hline
\end{tabular}


Table B - 24. Temperature $\left({ }^{\circ} \mathrm{C}\right)$ map of the west side of plate 1B5 at MOC 143A (70.0 EFPD cumulative).

\begin{tabular}{|c||c|c|r|r|r|r|r|r|r|r|}
\hline \multirow{2}{*}{$\begin{array}{c}\text { Length of } \\
\text { Plate (in) }\end{array}$} & \multicolumn{10}{|c|}{ Width of Plate (inches) } \\
\hline 22.625 & 59.18 & 56.68 & 56.74 & 56.82 & 56.86 & 56.85 & 56.77 & 56.62 & 56.71 & 58.52 \\
\hline 23.00 & 60.57 & 61.31 & 62.31 & 62.42 & 62.45 & 62.45 & 62.36 & 62.04 & 59.60 & 57.92 \\
\hline 24.00 & 65.00 & 96.40 & 100.11 & 100.24 & 100.27 & 100.27 & 100.19 & 99.90 & 81.92 & 60.33 \\
\hline 25.00 & 65.20 & 96.66 & 100.39 & 100.53 & 100.56 & 100.55 & 100.47 & 100.17 & 82.17 & 61.05 \\
\hline 26.00 & 65.34 & 96.78 & 100.51 & 100.65 & 100.68 & 100.68 & 100.59 & 100.28 & 82.40 & 62.50 \\
\hline 27.00 & 65.47 & 96.98 & 100.72 & 100.86 & 100.90 & 100.89 & 100.80 & 100.48 & 82.69 & 63.00 \\
\hline 28.00 & 65.62 & 97.43 & 101.20 & 101.34 & 101.38 & 101.37 & 101.28 & 100.96 & 82.93 & 62.54 \\
\hline 29.00 & 65.73 & 97.64 & 101.41 & 101.56 & 101.60 & 101.59 & 101.49 & 101.16 & 83.02 & 61.55 \\
\hline 30.00 & 65.71 & 96.98 & 100.69 & 100.84 & 100.88 & 100.87 & 100.77 & 100.44 & 82.64 & 61.13 \\
\hline 31.00 & 65.78 & 97.16 & 100.87 & 101.02 & 101.06 & 101.05 & 100.95 & 100.62 & 82.82 & 61.23 \\
\hline 32.00 & 65.70 & 96.32 & 99.95 & 100.10 & 100.14 & 100.14 & 100.03 & 99.70 & 82.33 & 61.01 \\
\hline 33.00 & 65.75 & 96.49 & 100.12 & 100.28 & 100.32 & 100.31 & 100.20 & 99.87 & 82.45 & 61.04 \\
\hline 34.00 & 65.57 & 95.15 & 98.67 & 98.82 & 98.86 & 98.86 & 98.74 & 98.41 & 81.65 & 61.56 \\
\hline 35.00 & 65.59 & 95.31 & 98.83 & 98.99 & 99.03 & 99.02 & 98.91 & 98.57 & 81.88 & 62.67 \\
\hline 36.00 & 65.44 & 94.37 & 97.80 & 97.96 & 98.00 & 97.99 & 97.87 & 97.53 & 81.42 & 63.22 \\
\hline 37.00 & 65.43 & 94.52 & 97.95 & 98.11 & 98.16 & 98.15 & 98.03 & 97.69 & 81.45 & 62.65 \\
\hline 38.00 & 65.04 & 92.07 & 95.23 & 95.39 & 95.44 & 95.43 & 95.30 & 94.97 & 79.81 & 61.33 \\
\hline 39.00 & 64.99 & 92.19 & 95.36 & 95.52 & 95.57 & 95.56 & 95.43 & 95.10 & 79.91 & 60.88 \\
\hline 40.00 & 64.55 & 89.52 & 92.37 & 92.51 & 92.56 & 92.55 & 92.43 & 92.12 & 78.20 & 60.66 \\
\hline 41.00 & 64.46 & 89.62 & 92.46 & 92.62 & 92.67 & 92.65 & 92.53 & 92.22 & 78.30 & 60.79 \\
\hline 42.00 & 63.84 & 86.04 & 88.60 & 88.75 & 88.79 & 88.78 & 88.66 & 88.36 & 75.95 & 60.20 \\
\hline 43.00 & 63.71 & 86.11 & 88.68 & 88.82 & 88.87 & 88.85 & 88.73 & 88.43 & 76.02 & 60.57 \\
\hline 44.00 & 60.47 & 61.03 & 61.44 & 61.58 & 61.63 & 61.61 & 61.47 & 61.19 & 60.50 & 59.44 \\
\hline 45.00 & 59.87 & 60.50 & 60.73 & 60.86 & 60.91 & 60.90 & 60.75 & 60.54 & 60.15 & 59.27 \\
\hline
\end{tabular}


Table B - 25. Temperature $\left({ }^{\circ} \mathrm{C}\right)$ map of the east side of plate 1T2 at MOC 143A (90.0 EFPD cumulative).

\begin{tabular}{|c||c|c|c|c|c|c|c|c|c|c|}
\hline \multirow{2}{*}{$\begin{array}{c}\text { Length of } \\
\text { Plate (in) }\end{array}$} & \multicolumn{10}{|c|}{ Width of Plate (inches) } \\
\hline & 0.05 & 0.25 & 0.50 & 0.75 & 1.00 & 1.25 & 1.50 & 1.75 & 2.00 & 2.24 \\
\hline 0.125 & 52.08 & 51.79 & 51.77 & 51.77 & 51.77 & 51.77 & 51.77 & 51.77 & 51.85 & 52.12 \\
\hline 1.00 & 53.19 & 52.39 & 52.50 & 52.52 & 52.52 & 52.52 & 52.51 & 52.46 & 52.38 & 53.08 \\
\hline 2.00 & 56.45 & 77.03 & 79.38 & 79.43 & 79.43 & 79.43 & 79.43 & 79.28 & 67.62 & 54.95 \\
\hline 3.00 & 56.81 & 77.24 & 79.61 & 79.66 & 79.67 & 79.66 & 79.66 & 79.50 & 67.85 & 55.16 \\
\hline 4.00 & 57.67 & 81.93 & 84.66 & 84.71 & 84.72 & 84.72 & 84.71 & 84.54 & 70.97 & 55.82 \\
\hline 5.00 & 57.95 & 82.09 & 84.81 & 84.87 & 84.88 & 84.88 & 84.87 & 84.69 & 71.14 & 56.01 \\
\hline 6.00 & 58.61 & 85.05 & 87.99 & 88.05 & 88.06 & 88.06 & 88.05 & 87.86 & 73.14 & 56.42 \\
\hline 7.00 & 58.87 & 85.20 & 88.14 & 88.20 & 88.21 & 88.21 & 88.20 & 88.01 & 73.31 & 56.61 \\
\hline 8.00 & 59.45 & 87.39 & 90.48 & 90.55 & 90.56 & 90.56 & 90.54 & 90.35 & 74.83 & 57.01 \\
\hline 9.00 & 59.71 & 87.54 & 90.63 & 90.70 & 90.71 & 90.71 & 90.69 & 90.49 & 75.17 & 58.04 \\
\hline 10.00 & 60.15 & 88.97 & 92.15 & 92.23 & 92.24 & 92.23 & 92.21 & 92.01 & 76.11 & 58.16 \\
\hline 11.00 & 60.39 & 89.12 & 92.30 & 92.37 & 92.39 & 92.38 & 92.36 & 92.15 & 76.20 & 57.98 \\
\hline 12.00 & 60.76 & 89.89 & 93.11 & 93.19 & 93.20 & 93.20 & 93.17 & 92.96 & 76.79 & 58.09 \\
\hline 13.00 & 60.99 & 90.04 & 93.25 & 93.33 & 93.35 & 93.35 & 93.32 & 93.11 & 76.97 & 58.36 \\
\hline 14.00 & 61.28 & 90.61 & 93.84 & 93.93 & 93.94 & 93.94 & 93.91 & 93.70 & 77.42 & 58.67 \\
\hline 15.00 & 61.50 & 90.76 & 93.99 & 94.08 & 94.09 & 94.09 & 94.06 & 93.84 & 77.57 & 58.79 \\
\hline 16.00 & 61.78 & 91.17 & 94.41 & 94.50 & 94.52 & 94.52 & 94.48 & 94.26 & 77.91 & 58.92 \\
\hline 17.00 & 61.99 & 91.31 & 94.56 & 94.65 & 94.67 & 94.67 & 94.63 & 94.40 & 78.18 & 59.83 \\
\hline 18.00 & 62.20 & 91.51 & 94.75 & 94.85 & 94.87 & 94.86 & 94.83 & 94.60 & 78.48 & 60.30 \\
\hline 19.00 & 62.39 & 91.66 & 94.90 & 94.99 & 95.01 & 95.01 & 94.97 & 94.74 & 78.55 & 60.30 \\
\hline 20.00 & 62.58 & 91.71 & 94.94 & 95.03 & 95.06 & 95.05 & 95.01 & 94.78 & 78.56 & 59.92 \\
\hline 21.00 & 62.73 & 91.83 & 95.06 & 95.16 & 95.18 & 95.18 & 95.13 & 94.90 & 78.69 & 59.86 \\
\hline 22.00 & 58.97 & 60.37 & 61.28 & 61.37 & 61.40 & 61.39 & 61.32 & 61.04 & 58.89 & 57.73 \\
\hline 22.50 & 57.81 & 56.23 & 56.26 & 56.32 & 56.35 & 56.34 & 56.28 & 56.17 & 56.38 & 57.78 \\
\hline
\end{tabular}


Table B - 26. Temperature $\left({ }^{\circ} \mathrm{C}\right)$ map of the west side of plate 1T2 at MOC 143A (90.0 EFPD cumulative).

\begin{tabular}{|c||c|c|c|c|c|c|c|c|c|c|}
\hline \multirow{2}{*}{$\begin{array}{c}\text { Length of } \\
\text { Plate (in) }\end{array}$} & \multicolumn{10}{|c|}{ Width of Plate (inches) } \\
\hline & 0.05 & 0.25 & 0.50 & 0.75 & 1.00 & 1.25 & 1.50 & 1.75 & 2.00 & 2.24 \\
\hline 0.125 & 52.33 & 51.79 & 51.77 & 51.77 & 51.77 & 51.77 & 51.77 & 51.77 & 51.85 & 52.31 \\
\hline 1.00 & 53.66 & 52.39 & 52.50 & 52.52 & 52.52 & 52.52 & 52.51 & 52.46 & 52.38 & 53.41 \\
\hline 2.00 & 56.94 & 77.03 & 79.38 & 79.43 & 79.44 & 79.44 & 79.43 & 79.29 & 67.62 & 54.94 \\
\hline 3.00 & 57.37 & 77.25 & 79.62 & 79.67 & 79.68 & 79.68 & 79.67 & 79.51 & 67.85 & 55.01 \\
\hline 4.00 & 58.27 & 81.95 & 84.67 & 84.73 & 84.73 & 84.73 & 84.72 & 84.55 & 70.98 & 55.67 \\
\hline 5.00 & 58.60 & 82.11 & 84.83 & 84.89 & 84.90 & 84.90 & 84.89 & 84.71 & 71.15 & 55.79 \\
\hline 6.00 & 59.30 & 85.08 & 88.02 & 88.08 & 88.09 & 88.09 & 88.08 & 87.89 & 73.15 & 56.20 \\
\hline 7.00 & 59.61 & 85.23 & 88.17 & 88.24 & 88.25 & 88.25 & 88.23 & 88.04 & 73.32 & 56.44 \\
\hline 8.00 & 60.24 & 87.44 & 90.53 & 90.60 & 90.60 & 90.60 & 90.59 & 90.38 & 74.85 & 56.81 \\
\hline 9.00 & 60.53 & 87.59 & 90.68 & 90.75 & 90.76 & 90.76 & 90.74 & 90.54 & 75.18 & 58.69 \\
\hline 10.00 & 61.01 & 89.03 & 92.21 & 92.29 & 92.30 & 92.30 & 92.27 & 92.06 & 76.14 & 58.62 \\
\hline 11.00 & 61.30 & 89.19 & 92.37 & 92.45 & 92.46 & 92.46 & 92.43 & 92.21 & 76.23 & 57.97 \\
\hline 12.00 & 61.70 & 89.97 & 93.19 & 93.27 & 93.28 & 93.28 & 93.25 & 93.03 & 76.83 & 57.78 \\
\hline 13.00 & 61.96 & 90.13 & 93.34 & 93.43 & 93.44 & 93.44 & 93.41 & 93.18 & 77.02 & 58.01 \\
\hline 14.00 & 62.28 & 90.70 & 93.94 & 94.03 & 94.05 & 94.05 & 94.01 & 93.78 & 77.47 & 58.41 \\
\hline 15.00 & 62.53 & 90.86 & 94.10 & 94.19 & 94.21 & 94.21 & 94.17 & 93.93 & 77.63 & 58.59 \\
\hline 16.00 & 62.84 & 91.28 & 94.54 & 94.63 & 94.65 & 94.65 & 94.60 & 94.37 & 77.98 & 58.72 \\
\hline 17.00 & 63.07 & 91.44 & 94.69 & 94.79 & 94.81 & 94.81 & 94.76 & 94.52 & 78.26 & 60.39 \\
\hline 18.00 & 63.30 & 91.65 & 94.90 & 95.00 & 95.02 & 95.02 & 94.97 & 94.73 & 78.56 & 61.12 \\
\hline 19.00 & 63.51 & 91.81 & 95.06 & 95.16 & 95.18 & 95.18 & 95.12 & 94.88 & 78.64 & 60.99 \\
\hline 20.00 & 63.71 & 91.87 & 95.11 & 95.21 & 95.24 & 95.23 & 95.18 & 94.93 & 78.66 & 59.96 \\
\hline 21.00 & 63.86 & 92.01 & 95.24 & 95.35 & 95.37 & 95.37 & 95.31 & 95.06 & 78.80 & 59.56 \\
\hline 22.00 & 60.17 & 60.53 & 61.43 & 61.53 & 61.56 & 61.56 & 61.48 & 61.18 & 59.00 & 57.63 \\
\hline 22.50 & 59.07 & 56.40 & 56.42 & 56.49 & 56.52 & 56.52 & 56.44 & 56.32 & 56.48 & 58.95 \\
\hline
\end{tabular}


Table B - 27. Temperature $\left({ }^{\circ} \mathrm{C}\right)$ map of the east side of plate 1B5 at MOC 143A (90.0 EFPD cumulative).

\begin{tabular}{|c||c|c|c|c|c|c|c|c|c|c|}
\hline \multirow{2}{*}{$\begin{array}{l}\text { Length of } \\
\text { Plate (in) }\end{array}$} & 0.05 & 0.25 & 0.50 & 0.75 & 1.00 & 1.25 & 1.50 & 1.75 & 2.00 & 2.24 \\
\hline 22.625 & 57.86 & 56.27 & 56.30 & 56.37 & 56.40 & 56.39 & 56.33 & 56.22 & 56.38 & 57.60 \\
\hline 23.00 & 59.06 & 60.36 & 61.23 & 61.33 & 61.35 & 61.35 & 61.28 & 61.01 & 58.91 & 57.82 \\
\hline 24.00 & 63.03 & 91.67 & 94.84 & 94.94 & 94.96 & 94.96 & 94.91 & 94.69 & 78.70 & 60.17 \\
\hline 25.00 & 63.21 & 91.90 & 95.08 & 95.18 & 95.21 & 95.20 & 95.15 & 94.92 & 78.93 & 60.53 \\
\hline 26.00 & 63.38 & 92.33 & 95.53 & 95.63 & 95.66 & 95.66 & 95.60 & 95.37 & 79.38 & 61.26 \\
\hline 27.00 & 63.51 & 92.48 & 95.68 & 95.79 & 95.82 & 95.81 & 95.76 & 95.52 & 79.64 & 61.58 \\
\hline 28.00 & 63.64 & 92.75 & 95.97 & 96.08 & 96.10 & 96.10 & 96.04 & 95.80 & 79.77 & 61.41 \\
\hline 29.00 & 63.75 & 92.91 & 96.13 & 96.26 & 96.29 & 96.28 & 96.21 & 95.96 & 79.83 & 61.04 \\
\hline 30.00 & 63.81 & 92.78 & 95.98 & 96.11 & 96.14 & 96.13 & 96.06 & 95.80 & 79.79 & 60.94 \\
\hline 31.00 & 63.89 & 92.91 & 96.14 & 96.27 & 96.30 & 96.29 & 96.22 & 95.95 & 79.95 & 61.08 \\
\hline 32.00 & 63.92 & 92.74 & 95.94 & 96.07 & 96.10 & 96.10 & 96.02 & 95.75 & 79.87 & 61.05 \\
\hline 33.00 & 63.99 & 92.87 & 96.10 & 96.22 & 96.26 & 96.25 & 96.18 & 95.90 & 79.99 & 61.04 \\
\hline 34.00 & 63.95 & 92.43 & 95.60 & 95.73 & 95.76 & 95.76 & 95.68 & 95.40 & 79.75 & 61.25 \\
\hline 35.00 & 64.00 & 92.56 & 95.75 & 95.88 & 95.91 & 95.91 & 95.83 & 95.55 & 79.96 & 61.77 \\
\hline 36.00 & 63.94 & 92.09 & 95.22 & 95.35 & 95.39 & 95.38 & 95.30 & 95.02 & 79.80 & 62.05 \\
\hline 37.00 & 63.96 & 92.21 & 95.36 & 95.50 & 95.53 & 95.53 & 95.44 & 95.16 & 79.81 & 61.78 \\
\hline 38.00 & 63.68 & 90.37 & 93.33 & 93.45 & 93.49 & 93.48 & 93.40 & 93.15 & 78.55 & 61.14 \\
\hline 39.00 & 63.68 & 90.48 & 93.44 & 93.56 & 93.60 & 93.59 & 93.52 & 93.26 & 78.64 & 60.96 \\
\hline 40.00 & 63.35 & 88.42 & 91.22 & 91.34 & 91.38 & 91.37 & 91.29 & 91.04 & 77.33 & 60.84 \\
\hline 41.00 & 63.31 & 88.51 & 91.31 & 91.44 & 91.47 & 91.46 & 91.39 & 91.13 & 77.42 & 60.88 \\
\hline 42.00 & 62.87 & 85.89 & 88.49 & 88.61 & 88.64 & 88.64 & 88.56 & 88.30 & 75.70 & 60.44 \\
\hline 43.00 & 62.80 & 85.96 & 88.56 & 88.68 & 88.72 & 88.71 & 88.63 & 88.38 & 75.78 & 60.56 \\
\hline 44.00 & 59.48 & 60.22 & 60.59 & 60.71 & 60.76 & 60.74 & 60.63 & 60.40 & 59.79 & 58.85 \\
\hline 45.00 & 58.89 & 59.67 & 59.86 & 59.97 & 60.01 & 60.00 & 59.89 & 59.73 & 59.43 & 58.57 \\
\hline
\end{tabular}


Table B - 28. Temperature $\left({ }^{\circ} \mathrm{C}\right)$ map of the west side of plate 1B5 at MOC 143A (90.0 EFPD cumulative).

\begin{tabular}{|c||c|c|c|c|c|c|c|c|c|c|}
\hline \multirow{2}{*}{$\begin{array}{c}\text { Length of } \\
\text { Plate (in) }\end{array}$} & \multicolumn{10}{|c|}{ Width of Plate (inches) } \\
\hline 22.625 & 59.03 & 56.44 & 56.47 & 56.54 & 56.58 & 56.57 & 56.49 & 56.37 & 56.49 & 58.40 \\
\hline 23.00 & 60.26 & 60.52 & 61.40 & 61.50 & 61.53 & 61.53 & 61.44 & 61.16 & 59.03 & 57.73 \\
\hline 24.00 & 64.17 & 91.89 & 95.06 & 95.17 & 95.19 & 95.19 & 95.13 & 94.88 & 78.84 & 59.87 \\
\hline 25.00 & 64.37 & 92.13 & 95.31 & 95.43 & 95.45 & 95.45 & 95.38 & 95.13 & 79.08 & 60.57 \\
\hline 26.00 & 64.54 & 92.58 & 95.78 & 95.89 & 95.92 & 95.91 & 95.84 & 95.59 & 79.54 & 61.98 \\
\hline 27.00 & 64.65 & 92.74 & 95.95 & 96.07 & 96.10 & 96.10 & 96.02 & 95.75 & 79.81 & 62.44 \\
\hline 28.00 & 64.78 & 93.02 & 96.26 & 96.39 & 96.42 & 96.42 & 96.33 & 96.05 & 79.94 & 61.99 \\
\hline 29.00 & 64.88 & 93.19 & 96.46 & 96.59 & 96.62 & 96.62 & 96.53 & 96.24 & 80.02 & 61.05 \\
\hline 30.00 & 64.92 & 93.06 & 96.32 & 96.45 & 96.49 & 96.48 & 96.39 & 96.10 & 79.98 & 60.68 \\
\hline 31.00 & 64.98 & 93.21 & 96.49 & 96.63 & 96.66 & 96.65 & 96.56 & 96.26 & 80.15 & 60.77 \\
\hline 32.00 & 64.99 & 93.05 & 96.31 & 96.44 & 96.48 & 96.47 & 96.38 & 96.07 & 80.09 & 60.61 \\
\hline 33.00 & 65.03 & 93.19 & 96.47 & 96.61 & 96.65 & 96.64 & 96.54 & 96.23 & 80.21 & 60.63 \\
\hline 34.00 & 64.98 & 92.76 & 95.99 & 96.13 & 96.17 & 96.16 & 96.06 & 95.75 & 79.98 & 61.20 \\
\hline 35.00 & 64.99 & 92.90 & 96.15 & 96.29 & 96.33 & 96.32 & 96.22 & 95.91 & 80.20 & 62.26 \\
\hline 36.00 & 64.91 & 92.44 & 95.63 & 95.78 & 95.82 & 95.81 & 95.70 & 95.39 & 80.04 & 62.82 \\
\hline 37.00 & 64.90 & 92.57 & 95.78 & 95.93 & 95.97 & 95.96 & 95.85 & 95.54 & 80.06 & 62.28 \\
\hline 38.00 & 64.59 & 90.75 & 93.73 & 93.87 & 93.91 & 93.90 & 93.79 & 93.49 & 78.81 & 61.05 \\
\hline 39.00 & 64.54 & 90.87 & 93.86 & 94.01 & 94.05 & 94.04 & 93.92 & 93.61 & 78.91 & 60.60 \\
\hline 40.00 & 64.18 & 88.82 & 91.63 & 91.77 & 91.80 & 91.79 & 91.69 & 91.40 & 77.61 & 60.44 \\
\hline 41.00 & 64.10 & 88.92 & 91.73 & 91.87 & 91.91 & 91.90 & 91.79 & 91.50 & 77.71 & 60.56 \\
\hline 42.00 & 63.62 & 86.31 & 88.92 & 89.06 & 89.10 & 89.08 & 88.97 & 88.69 & 75.99 & 60.06 \\
\hline 43.00 & 63.50 & 86.39 & 89.00 & 89.14 & 89.18 & 89.17 & 89.05 & 88.76 & 76.07 & 60.42 \\
\hline 44.00 & 60.16 & 60.56 & 60.95 & 61.07 & 61.12 & 61.11 & 60.97 & 60.71 & 60.06 & 59.20 \\
\hline 45.00 & 59.54 & 60.01 & 60.22 & 60.33 & 60.38 & 60.36 & 60.23 & 60.04 & 59.70 & 59.00 \\
\hline
\end{tabular}


Table B - 29. Temperature $\left({ }^{\circ} \mathrm{C}\right)$ map of the east side of plate $1 \mathrm{~T} 2$ at EOC 143A (100.9 EFPD cumulative).

\begin{tabular}{|c||c|c|c|c|c|c|c|c|c|c|}
\hline \multirow{2}{*}{$\begin{array}{c}\text { Length of } \\
\text { Plate (in) }\end{array}$} & \multicolumn{10}{|c|}{ Width of Plate (inches) } \\
\hline & 0.05 & 0.25 & 0.50 & 0.75 & 1.00 & 1.25 & 1.50 & 1.75 & 2.00 & 2.24 \\
\hline 0.125 & 52.08 & 51.79 & 51.77 & 51.77 & 51.77 & 51.77 & 51.77 & 51.77 & 51.85 & 52.12 \\
\hline 1.00 & 53.17 & 52.37 & 52.48 & 52.49 & 52.49 & 52.49 & 52.49 & 52.43 & 52.37 & 53.07 \\
\hline 2.00 & 56.33 & 76.26 & 78.55 & 78.59 & 78.60 & 78.60 & 78.59 & 78.45 & 67.12 & 54.88 \\
\hline 3.00 & 56.68 & 76.46 & 78.77 & 78.82 & 78.82 & 78.82 & 78.82 & 78.66 & 67.35 & 55.08 \\
\hline 4.00 & 57.48 & 80.71 & 83.34 & 83.39 & 83.40 & 83.39 & 83.39 & 83.22 & 70.17 & 55.70 \\
\hline 5.00 & 57.74 & 80.85 & 83.49 & 83.54 & 83.55 & 83.55 & 83.54 & 83.37 & 70.33 & 55.88 \\
\hline 6.00 & 58.34 & 83.42 & 86.24 & 86.30 & 86.31 & 86.31 & 86.30 & 86.12 & 72.06 & 56.26 \\
\hline 7.00 & 58.59 & 83.56 & 86.38 & 86.44 & 86.45 & 86.45 & 86.44 & 86.25 & 72.22 & 56.45 \\
\hline 8.00 & 59.13 & 85.48 & 88.43 & 88.50 & 88.50 & 88.50 & 88.49 & 88.30 & 73.55 & 56.81 \\
\hline 9.00 & 59.38 & 85.63 & 88.57 & 88.64 & 88.65 & 88.65 & 88.63 & 88.44 & 73.88 & 57.81 \\
\hline 10.00 & 59.74 & 86.59 & 89.59 & 89.66 & 89.67 & 89.67 & 89.65 & 89.45 & 74.50 & 57.90 \\
\hline 11.00 & 59.98 & 86.73 & 89.73 & 89.80 & 89.81 & 89.81 & 89.79 & 89.59 & 74.59 & 57.72 \\
\hline 12.00 & 60.30 & 87.25 & 90.27 & 90.34 & 90.36 & 90.36 & 90.33 & 90.13 & 75.00 & 57.81 \\
\hline 13.00 & 60.52 & 87.39 & 90.41 & 90.48 & 90.50 & 90.50 & 90.47 & 90.26 & 75.18 & 58.07 \\
\hline 14.00 & 60.77 & 87.72 & 90.75 & 90.83 & 90.84 & 90.84 & 90.81 & 90.60 & 75.46 & 58.34 \\
\hline 15.00 & 60.98 & 87.86 & 90.88 & 90.97 & 90.98 & 90.98 & 90.95 & 90.74 & 75.60 & 58.46 \\
\hline 16.00 & 61.22 & 88.06 & 91.09 & 91.17 & 91.19 & 91.19 & 91.15 & 90.94 & 75.80 & 58.57 \\
\hline 17.00 & 61.42 & 88.20 & 91.22 & 91.31 & 91.33 & 91.32 & 91.29 & 91.07 & 76.06 & 59.45 \\
\hline 18.00 & 61.61 & 88.34 & 91.36 & 91.44 & 91.46 & 91.46 & 91.42 & 91.21 & 76.32 & 59.89 \\
\hline 19.00 & 61.79 & 88.48 & 91.49 & 91.58 & 91.60 & 91.60 & 91.56 & 91.34 & 76.37 & 59.89 \\
\hline 20.00 & 61.97 & 88.49 & 91.49 & 91.58 & 91.60 & 91.60 & 91.56 & 91.34 & 76.35 & 59.53 \\
\hline 21.00 & 62.12 & 88.61 & 91.60 & 91.70 & 91.72 & 91.71 & 91.67 & 91.45 & 76.47 & 59.46 \\
\hline 22.00 & 58.70 & 59.73 & 60.54 & 60.62 & 60.65 & 60.64 & 60.58 & 60.32 & 58.40 & 57.53 \\
\hline 22.50 & 57.65 & 55.96 & 55.96 & 56.02 & 56.04 & 56.04 & 55.98 & 55.89 & 56.13 & 57.64 \\
\hline
\end{tabular}


Table B - 30. Temperature $\left({ }^{\circ} \mathrm{C}\right)$ map of the west side of plate 1T2 at EOC 143A (100.9 EFPD cumulative).

\begin{tabular}{|c|c|c|c|c|c|c|c|c|c|c|}
\hline \multirow{2}{*}{$\begin{array}{c}\text { Length of } \\
\text { Plate (in) }\end{array}$} & \multicolumn{10}{|c|}{ Width of Plate (inches) } \\
\hline & 0.05 & 0.25 & 0.50 & 0.75 & 1.00 & 1.25 & 1.50 & 1.75 & 2.00 & 2.24 \\
\hline 0.125 & 52.33 & 51.79 & 51.77 & 51.77 & 51.77 & 51.77 & 51.77 & 51.77 & 51.85 & 52.31 \\
\hline 1.00 & 53.65 & 52.38 & 52.48 & 52.49 & 52.49 & 52.49 & 52.49 & 52.44 & 52.37 & 53.40 \\
\hline 2.00 & 56.82 & 76.26 & 78.55 & 78.60 & 78.60 & 78.60 & 78.60 & 78.46 & 67.12 & 54.87 \\
\hline 3.00 & 57.24 & 76.48 & 78.78 & 78.83 & 78.83 & 78.83 & 78.83 & 78.67 & 67.35 & 54.93 \\
\hline 4.00 & 58.08 & 80.72 & 83.35 & 83.41 & 83.41 & 83.41 & 83.40 & 83.23 & 70.17 & 55.56 \\
\hline 5.00 & 58.40 & 80.88 & 83.51 & 83.56 & 83.57 & 83.57 & 83.56 & 83.38 & 70.33 & 55.68 \\
\hline 6.00 & 59.03 & 83.45 & 86.27 & 86.33 & 86.34 & 86.34 & 86.32 & 86.14 & 72.07 & 56.06 \\
\hline 7.00 & 59.34 & 83.60 & 86.42 & 86.48 & 86.49 & 86.49 & 86.47 & 86.28 & 72.23 & 56.29 \\
\hline 8.00 & 59.92 & 85.53 & 88.47 & 88.54 & 88.55 & 88.55 & 88.53 & 88.34 & 73.57 & 56.64 \\
\hline 9.00 & 60.21 & 85.68 & 88.62 & 88.69 & 88.70 & 88.70 & 88.68 & 88.48 & 73.89 & 58.47 \\
\hline 10.00 & 60.61 & 86.65 & 89.65 & 89.73 & 89.74 & 89.74 & 89.71 & 89.51 & 74.53 & 58.36 \\
\hline 11.00 & 60.88 & 86.80 & 89.80 & 89.88 & 89.89 & 89.89 & 89.86 & 89.65 & 74.62 & 57.74 \\
\hline 12.00 & 61.24 & 87.32 & 90.35 & 90.43 & 90.44 & 90.44 & 90.41 & 90.20 & 75.04 & 57.53 \\
\hline 13.00 & 61.49 & 87.48 & 90.50 & 90.58 & 90.59 & 90.59 & 90.56 & 90.34 & 75.23 & 57.75 \\
\hline 14.00 & 61.77 & 87.82 & 90.85 & 90.93 & 90.95 & 90.95 & 90.91 & 90.69 & 75.52 & 58.13 \\
\hline 15.00 & 62.01 & 87.97 & 91.00 & 91.08 & 91.10 & 91.10 & 91.06 & 90.83 & 75.67 & 58.30 \\
\hline 16.00 & 62.28 & 88.19 & 91.21 & 91.30 & 91.32 & 91.32 & 91.28 & 91.05 & 75.87 & 58.40 \\
\hline 17.00 & 62.50 & 88.34 & 91.36 & 91.45 & 91.47 & 91.47 & 91.42 & 91.19 & 76.14 & 60.02 \\
\hline 18.00 & 62.71 & 88.48 & 91.50 & 91.60 & 91.62 & 91.61 & 91.57 & 91.34 & 76.40 & 60.72 \\
\hline 19.00 & 62.91 & 88.63 & 91.65 & 91.75 & 91.77 & 91.76 & 91.71 & 91.48 & 76.47 & 60.60 \\
\hline 20.00 & 63.10 & 88.66 & 91.66 & 91.76 & 91.78 & 91.78 & 91.73 & 91.49 & 76.46 & 59.60 \\
\hline 21.00 & 63.25 & 88.79 & 91.79 & 91.89 & 91.91 & 91.91 & 91.85 & 91.61 & 76.59 & 59.21 \\
\hline 22.00 & 59.88 & 59.88 & 60.69 & 60.78 & 60.81 & 60.81 & 60.73 & 60.46 & 58.52 & 57.46 \\
\hline 22.50 & 58.89 & 56.12 & 56.13 & 56.19 & 56.22 & 56.21 & 56.14 & 56.03 & 56.24 & 58.80 \\
\hline
\end{tabular}


Table B - 31. Temperature $\left({ }^{\circ} \mathrm{C}\right)$ map of the east side of plate 1B5 at EOC 143A (100.9 EFPD cumulative).

\begin{tabular}{|c||c|c|c|c|c|c|c|c|c|c|}
\hline \multirow{2}{*}{$\begin{array}{l}\text { Length of } \\
\text { Plate (in) }\end{array}$} & 0.05 & 0.25 & 0.50 & 0.75 & 1.00 & 1.25 & 1.50 & 1.75 & 2.00 & 2.24 \\
\hline 22.625 & 57.69 & 56.00 & 56.00 & 56.06 & 56.09 & 56.08 & 56.02 & 55.93 & 56.13 & 57.47 \\
\hline 23.00 & 58.78 & 59.72 & 60.50 & 60.58 & 60.61 & 60.60 & 60.54 & 60.29 & 58.42 & 57.62 \\
\hline 24.00 & 62.40 & 88.45 & 91.39 & 91.48 & 91.51 & 91.50 & 91.46 & 91.24 & 76.48 & 59.76 \\
\hline 25.00 & 62.57 & 88.68 & 91.63 & 91.72 & 91.75 & 91.74 & 91.69 & 91.48 & 76.71 & 60.11 \\
\hline 26.00 & 62.75 & 89.19 & 92.17 & 92.27 & 92.29 & 92.29 & 92.24 & 92.02 & 77.21 & 60.82 \\
\hline 27.00 & 62.86 & 89.33 & 92.31 & 92.41 & 92.44 & 92.43 & 92.38 & 92.16 & 77.46 & 61.13 \\
\hline 28.00 & 62.99 & 89.62 & 92.61 & 92.71 & 92.73 & 92.73 & 92.67 & 92.45 & 77.59 & 60.96 \\
\hline 29.00 & 63.09 & 89.76 & 92.75 & 92.86 & 92.88 & 92.88 & 92.82 & 92.59 & 77.64 & 60.60 \\
\hline 30.00 & 63.16 & 89.75 & 92.74 & 92.84 & 92.86 & 92.86 & 92.80 & 92.57 & 77.68 & 60.51 \\
\hline 31.00 & 63.23 & 89.88 & 92.86 & 92.97 & 92.99 & 92.99 & 92.93 & 92.70 & 77.83 & 60.65 \\
\hline 32.00 & 63.28 & 89.87 & 92.84 & 92.94 & 92.97 & 92.97 & 92.91 & 92.68 & 77.86 & 60.63 \\
\hline 33.00 & 63.34 & 89.99 & 92.97 & 93.07 & 93.10 & 93.09 & 93.03 & 92.80 & 77.96 & 60.62 \\
\hline 34.00 & 63.33 & 89.73 & 92.68 & 92.79 & 92.82 & 92.81 & 92.75 & 92.51 & 77.84 & 60.83 \\
\hline 35.00 & 63.36 & 89.85 & 92.80 & 92.91 & 92.94 & 92.94 & 92.87 & 92.64 & 78.04 & 61.32 \\
\hline 36.00 & 63.33 & 89.57 & 92.49 & 92.60 & 92.63 & 92.62 & 92.56 & 92.32 & 77.99 & 61.60 \\
\hline 37.00 & 63.35 & 89.68 & 92.61 & 92.72 & 92.75 & 92.74 & 92.68 & 92.44 & 78.00 & 61.35 \\
\hline 38.00 & 63.12 & 88.27 & 91.08 & 91.20 & 91.23 & 91.22 & 91.15 & 90.91 & 77.02 & 60.76 \\
\hline 39.00 & 63.11 & 88.37 & 91.19 & 91.30 & 91.33 & 91.33 & 91.26 & 91.02 & 77.11 & 60.59 \\
\hline 40.00 & 62.84 & 86.68 & 89.36 & 89.47 & 89.50 & 89.50 & 89.43 & 89.19 & 76.04 & 60.48 \\
\hline 41.00 & 62.80 & 86.77 & 89.45 & 89.57 & 89.60 & 89.59 & 89.52 & 89.28 & 76.13 & 60.52 \\
\hline 42.00 & 62.40 & 84.44 & 86.94 & 87.06 & 87.09 & 87.08 & 87.01 & 86.77 & 74.61 & 60.12 \\
\hline 43.00 & 62.33 & 84.51 & 87.02 & 87.13 & 87.16 & 87.16 & 87.08 & 86.84 & 74.68 & 60.22 \\
\hline 44.00 & 59.12 & 59.67 & 60.01 & 60.12 & 60.16 & 60.15 & 60.05 & 59.83 & 59.29 & 58.57 \\
\hline 45.00 & 58.55 & 59.14 & 59.31 & 59.40 & 59.44 & 59.43 & 59.33 & 59.18 & 58.94 & 58.29 \\
\hline
\end{tabular}


Table B - 32. Temperature $\left({ }^{\circ} \mathrm{C}\right)$ map of the west side of plate 1B5 at EOC 143A (100.9 EFPD cumulative).

\begin{tabular}{|c|c|c|c|c|c|c|c|c|c|c|}
\hline \multirow{2}{*}{$\begin{array}{c}\text { Length of } \\
\text { Plate (in) }\end{array}$} & \multicolumn{10}{|c|}{ Width of Plate (inches) } \\
\hline & 0.05 & 0.25 & 0.50 & 0.75 & 1.00 & 1.25 & 1.50 & 1.75 & 2.00 & 2.24 \\
\hline 22.625 & 58.85 & 56.16 & 56.17 & 56.23 & 56.26 & 56.26 & 56.19 & 56.08 & 56.24 & 58.27 \\
\hline 23.00 & 59.97 & 59.88 & 60.67 & 60.76 & 60.78 & 60.78 & 60.70 & 60.44 & 58.55 & 57.55 \\
\hline 24.00 & 63.54 & 88.67 & 91.61 & 91.72 & 91.74 & 91.74 & 91.68 & 91.44 & 76.63 & 59.52 \\
\hline 25.00 & 63.73 & 88.91 & 91.87 & 91.97 & 91.99 & 91.99 & 91.93 & 91.69 & 76.87 & 60.19 \\
\hline 26.00 & 63.89 & 89.44 & 92.42 & 92.53 & 92.55 & 92.55 & 92.48 & 92.24 & 77.37 & 61.55 \\
\hline 27.00 & 64.00 & 89.59 & 92.58 & 92.68 & 92.71 & 92.70 & 92.64 & 92.39 & 77.62 & 61.98 \\
\hline 28.00 & 64.12 & 89.89 & 92.88 & 92.99 & 93.02 & 93.01 & 92.94 & 92.69 & 77.77 & 61.56 \\
\hline 29.00 & 64.21 & 90.05 & 93.04 & 93.15 & 93.18 & 93.18 & 93.10 & 92.85 & 77.84 & 60.65 \\
\hline 30.00 & 64.26 & 90.05 & 93.04 & 93.15 & 93.18 & 93.17 & 93.10 & 92.84 & 77.88 & 60.30 \\
\hline 31.00 & 64.32 & 90.19 & 93.18 & 93.29 & 93.32 & 93.31 & 93.24 & 92.98 & 78.04 & 60.38 \\
\hline 32.00 & 64.34 & 90.18 & 93.17 & 93.28 & 93.31 & 93.30 & 93.22 & 92.97 & 78.08 & 60.24 \\
\hline 33.00 & 64.38 & 90.32 & 93.30 & 93.42 & 93.45 & 93.44 & 93.36 & 93.10 & 78.19 & 60.26 \\
\hline 34.00 & 64.34 & 90.07 & 93.03 & 93.15 & 93.18 & 93.17 & 93.09 & 92.83 & 78.08 & 60.81 \\
\hline 35.00 & 64.35 & 90.20 & 93.16 & 93.28 & 93.31 & 93.31 & 93.22 & 92.96 & 78.29 & 61.83 \\
\hline 36.00 & 64.29 & 89.93 & 92.86 & 92.98 & 93.01 & 93.00 & 92.92 & 92.65 & 78.24 & 62.37 \\
\hline 37.00 & 64.27 & 90.05 & 92.99 & 93.11 & 93.14 & 93.13 & 93.04 & 92.78 & 78.26 & 61.84 \\
\hline 38.00 & 64.02 & 88.65 & 91.48 & 91.60 & 91.63 & 91.62 & 91.53 & 91.26 & 77.29 & 60.70 \\
\hline 39.00 & 63.97 & 88.76 & 91.59 & 91.72 & 91.75 & 91.74 & 91.65 & 91.37 & 77.39 & 60.26 \\
\hline 40.00 & 63.66 & 87.08 & 89.78 & 89.90 & 89.93 & 89.92 & 89.83 & 89.56 & 76.33 & 60.12 \\
\hline 41.00 & 63.58 & 87.17 & 89.88 & 90.00 & 90.04 & 90.02 & 89.93 & 89.65 & 76.42 & 60.24 \\
\hline 42.00 & 63.14 & 84.86 & 87.38 & 87.50 & 87.54 & 87.53 & 87.43 & 87.16 & 74.91 & 59.77 \\
\hline 43.00 & 63.01 & 84.94 & 87.46 & 87.58 & 87.62 & 87.61 & 87.51 & 87.23 & 74.98 & 60.11 \\
\hline 44.00 & 59.80 & 60.01 & 60.37 & 60.48 & 60.52 & 60.51 & 60.39 & 60.14 & 59.56 & 58.92 \\
\hline 45.00 & 59.19 & 59.48 & 59.66 & 59.76 & 59.81 & 59.79 & 59.67 & 59.50 & 59.21 & 58.71 \\
\hline
\end{tabular}


Table B - 33. Temperature $\left({ }^{\circ} \mathrm{C}\right)$ map of the east side of plate 1T2 at BOC 143B (100.9 EFPD cumulative).

\begin{tabular}{|c||c|c|c|c|c|c|c|c|c|c|}
\hline \multirow{2}{*}{$\begin{array}{c}\text { Length of } \\
\text { Plate (in) }\end{array}$} & \multicolumn{10}{|c|}{ Width of Plate (inches) } \\
\hline & 0.05 & 0.25 & 0.50 & 0.75 & 1.00 & 1.25 & 1.50 & 1.75 & 2.00 & 2.24 \\
\hline 0.125 & 52.09 & 51.80 & 51.77 & 51.77 & 51.77 & 51.77 & 51.77 & 51.77 & 51.85 & 52.13 \\
\hline 1.00 & 53.34 & 52.25 & 52.29 & 52.30 & 52.30 & 52.30 & 52.30 & 52.27 & 52.31 & 53.23 \\
\hline 2.00 & 56.78 & 69.62 & 71.30 & 71.33 & 71.34 & 71.33 & 71.33 & 71.21 & 63.04 & 55.42 \\
\hline 3.00 & 57.20 & 69.85 & 71.53 & 71.57 & 71.57 & 71.57 & 71.56 & 71.44 & 63.29 & 55.69 \\
\hline 4.00 & 58.07 & 72.47 & 74.35 & 74.40 & 74.40 & 74.40 & 74.39 & 74.26 & 65.07 & 56.41 \\
\hline 5.00 & 58.41 & 72.68 & 74.56 & 74.61 & 74.62 & 74.62 & 74.60 & 74.46 & 65.29 & 56.66 \\
\hline 6.00 & 59.32 & 75.17 & 77.24 & 77.30 & 77.31 & 77.31 & 77.29 & 77.13 & 66.98 & 57.29 \\
\hline 7.00 & 59.66 & 75.41 & 77.47 & 77.53 & 77.55 & 77.55 & 77.52 & 77.36 & 67.23 & 57.55 \\
\hline 8.00 & 60.59 & 77.54 & 79.75 & 79.83 & 79.84 & 79.84 & 79.81 & 79.64 & 68.74 & 58.22 \\
\hline 9.00 & 60.92 & 77.80 & 80.01 & 80.09 & 80.10 & 80.10 & 80.07 & 79.89 & 69.17 & 59.30 \\
\hline 10.00 & 61.63 & 78.88 & 81.15 & 81.24 & 81.26 & 81.26 & 81.21 & 81.03 & 69.93 & 59.67 \\
\hline 11.00 & 61.97 & 79.16 & 81.43 & 81.52 & 81.54 & 81.54 & 81.49 & 81.30 & 70.14 & 59.57 \\
\hline 12.00 & 62.85 & 81.14 & 83.53 & 83.63 & 83.65 & 83.65 & 83.60 & 83.39 & 71.56 & 60.07 \\
\hline 13.00 & 63.19 & 81.44 & 83.83 & 83.93 & 83.96 & 83.96 & 83.90 & 83.69 & 71.89 & 60.42 \\
\hline 14.00 & 63.66 & 81.61 & 83.99 & 84.10 & 84.13 & 84.13 & 84.06 & 83.84 & 72.14 & 60.89 \\
\hline 15.00 & 64.00 & 81.92 & 84.30 & 84.41 & 84.45 & 84.45 & 84.37 & 84.15 & 72.44 & 61.11 \\
\hline 16.00 & 64.57 & 82.90 & 85.33 & 85.45 & 85.49 & 85.49 & 85.41 & 85.17 & 73.21 & 61.45 \\
\hline 17.00 & 64.90 & 83.23 & 85.65 & 85.78 & 85.83 & 85.83 & 85.74 & 85.50 & 73.66 & 62.48 \\
\hline 18.00 & 65.35 & 83.89 & 86.35 & 86.49 & 86.53 & 86.53 & 86.43 & 86.19 & 74.32 & 63.12 \\
\hline 19.00 & 65.69 & 84.23 & 86.69 & 86.83 & 86.88 & 86.88 & 86.78 & 86.52 & 74.57 & 63.25 \\
\hline 20.00 & 66.22 & 85.08 & 87.57 & 87.72 & 87.77 & 87.77 & 87.66 & 87.40 & 75.16 & 63.13 \\
\hline 21.00 & 66.52 & 85.40 & 87.89 & 88.05 & 88.10 & 88.10 & 87.99 & 87.72 & 75.48 & 63.16 \\
\hline 22.00 & 61.63 & 61.74 & 62.41 & 62.56 & 62.62 & 62.62 & 62.48 & 62.19 & 60.70 & 59.92 \\
\hline 22.50 & 59.84 & 58.81 & 58.88 & 59.01 & 59.08 & 59.07 & 58.93 & 58.77 & 58.90 & 59.45 \\
\hline
\end{tabular}


Table B - 34. Temperature $\left({ }^{\circ} \mathrm{C}\right)$ map of the west side of plate 1T2 at BOC 143B (100.9 EFPD cumulative).

\begin{tabular}{|c|c|c|c|c|c|c|c|c|c|c|}
\hline \multirow{2}{*}{$\begin{array}{c}\text { Length of } \\
\text { Plate (in) }\end{array}$} & \multicolumn{10}{|c|}{ Width of Plate (inches) } \\
\hline & 0.05 & 0.25 & 0.50 & 0.75 & 1.00 & 1.25 & 1.50 & 1.75 & 2.00 & 2.24 \\
\hline 0.125 & 52.34 & 51.80 & 51.77 & 51.77 & 51.77 & 51.77 & 51.77 & 51.77 & 51.85 & 52.33 \\
\hline 1.00 & 53.67 & 52.25 & 52.29 & 52.30 & 52.30 & 52.30 & 52.30 & 52.26 & 52.31 & 53.47 \\
\hline 2.00 & 56.44 & 69.59 & 71.27 & 71.30 & 71.31 & 71.31 & 71.30 & 71.19 & 63.02 & 54.96 \\
\hline 3.00 & 56.87 & 69.78 & 71.45 & 71.49 & 71.50 & 71.50 & 71.49 & 71.37 & 63.24 & 55.05 \\
\hline 4.00 & 57.59 & 72.35 & 74.22 & 74.27 & 74.27 & 74.27 & 74.26 & 74.13 & 64.97 & 55.67 \\
\hline 5.00 & 57.94 & 72.49 & 74.37 & 74.41 & 74.42 & 74.42 & 74.41 & 74.27 & 65.14 & 55.83 \\
\hline 6.00 & 58.68 & 74.92 & 76.98 & 77.03 & 77.03 & 77.03 & 77.02 & 76.87 & 66.77 & 56.34 \\
\hline 7.00 & 59.06 & 75.09 & 77.15 & 77.20 & 77.20 & 77.20 & 77.19 & 77.04 & 66.97 & 56.62 \\
\hline 8.00 & 59.75 & 77.11 & 79.31 & 79.37 & 79.37 & 79.37 & 79.36 & 79.20 & 68.38 & 57.11 \\
\hline 9.00 & 60.08 & 77.27 & 79.46 & 79.52 & 79.53 & 79.53 & 79.51 & 79.35 & 68.72 & 59.01 \\
\hline 10.00 & 60.62 & 78.26 & 80.50 & 80.57 & 80.58 & 80.57 & 80.55 & 80.38 & 69.40 & 59.07 \\
\hline 11.00 & 60.94 & 78.42 & 80.66 & 80.73 & 80.74 & 80.74 & 80.71 & 80.54 & 69.51 & 58.50 \\
\hline 12.00 & 61.63 & 80.27 & 82.64 & 82.71 & 82.72 & 82.72 & 82.69 & 82.51 & 70.83 & 58.52 \\
\hline 13.00 & 61.95 & 80.45 & 82.81 & 82.88 & 82.89 & 82.89 & 82.86 & 82.68 & 71.05 & 58.80 \\
\hline 14.00 & 62.29 & 80.49 & 82.83 & 82.90 & 82.92 & 82.91 & 82.88 & 82.70 & 71.19 & 59.28 \\
\hline 15.00 & 62.59 & 80.67 & 83.00 & 83.07 & 83.09 & 83.09 & 83.05 & 82.86 & 71.37 & 59.51 \\
\hline 16.00 & 63.04 & 81.50 & 83.87 & 83.95 & 83.96 & 83.96 & 83.92 & 83.73 & 72.01 & 59.76 \\
\hline 17.00 & 63.33 & 81.68 & 84.04 & 84.13 & 84.14 & 84.14 & 84.10 & 83.90 & 72.33 & 61.55 \\
\hline 18.00 & 63.68 & 82.20 & 84.59 & 84.67 & 84.69 & 84.69 & 84.64 & 84.45 & 72.86 & 62.37 \\
\hline 19.00 & 63.95 & 82.39 & 84.77 & 84.85 & 84.87 & 84.87 & 84.82 & 84.62 & 72.99 & 62.36 \\
\hline 20.00 & 64.35 & 83.06 & 85.47 & 85.56 & 85.58 & 85.58 & 85.53 & 85.32 & 73.43 & 61.45 \\
\hline 21.00 & 64.57 & 83.23 & 85.64 & 85.73 & 85.75 & 85.75 & 85.69 & 85.49 & 73.62 & 61.09 \\
\hline 22.00 & 61.09 & 59.96 & 60.55 & 60.65 & 60.69 & 60.68 & 60.59 & 60.36 & 58.96 & 58.83 \\
\hline 22.50 & 59.93 & 56.95 & 56.94 & 57.02 & 57.06 & 57.05 & 56.96 & 56.86 & 57.07 & 60.00 \\
\hline
\end{tabular}


Table B - 35. Temperature $\left({ }^{\circ} \mathrm{C}\right)$ map of the east side of plate 1B5 at BOC 143B (100.9 EFPD cumulative).

\begin{tabular}{|c||c|c|c|c|c|c|c|c|c|c|}
\hline \multirow{2}{*}{$\begin{array}{l}\text { Length of } \\
\text { Plate (in) }\end{array}$} & 0.05 & 0.25 & 0.50 & 0.75 & 1.00 & 1.25 & 1.50 & 1.75 & 2.00 & 2.24 \\
\hline 22.625 & 59.95 & 58.86 & 58.93 & 59.07 & 59.13 & 59.13 & 58.99 & 58.82 & 58.92 & 59.31 \\
\hline 23.00 & 61.47 & 61.89 & 62.56 & 62.71 & 62.77 & 62.77 & 62.63 & 62.35 & 60.84 & 59.81 \\
\hline 24.00 & 66.11 & 85.83 & 88.33 & 88.49 & 88.55 & 88.55 & 88.42 & 88.15 & 75.91 & 62.90 \\
\hline 25.00 & 66.35 & 86.15 & 88.65 & 88.82 & 88.88 & 88.88 & 88.75 & 88.47 & 76.23 & 63.31 \\
\hline 26.00 & 66.49 & 86.30 & 88.80 & 88.97 & 89.03 & 89.03 & 88.90 & 88.61 & 76.53 & 64.04 \\
\hline 27.00 & 66.66 & 86.57 & 89.07 & 89.25 & 89.31 & 89.31 & 89.17 & 88.88 & 76.90 & 64.37 \\
\hline 28.00 & 66.73 & 86.30 & 88.76 & 88.94 & 89.01 & 89.01 & 88.86 & 88.57 & 76.71 & 64.19 \\
\hline 29.00 & 66.91 & 86.60 & 89.06 & 89.25 & 89.32 & 89.31 & 89.17 & 88.87 & 76.90 & 63.88 \\
\hline 30.00 & 67.00 & 86.75 & 89.20 & 89.39 & 89.46 & 89.46 & 89.31 & 89.01 & 77.07 & 63.79 \\
\hline 31.00 & 67.14 & 87.01 & 89.47 & 89.66 & 89.73 & 89.73 & 89.57 & 89.27 & 77.34 & 63.98 \\
\hline 32.00 & 67.12 & 86.81 & 89.24 & 89.44 & 89.52 & 89.51 & 89.35 & 89.04 & 77.29 & 63.91 \\
\hline 33.00 & 67.25 & 87.09 & 89.54 & 89.75 & 89.83 & 89.82 & 89.65 & 89.32 & 77.53 & 63.97 \\
\hline 34.00 & 67.21 & 87.12 & 89.57 & 89.78 & 89.86 & 89.86 & 89.68 & 89.35 & 77.62 & 64.15 \\
\hline 35.00 & 67.30 & 87.35 & 89.83 & 90.04 & 90.13 & 90.12 & 89.94 & 89.60 & 77.94 & 64.70 \\
\hline 36.00 & 67.02 & 86.24 & 88.59 & 88.81 & 88.89 & 88.89 & 88.70 & 88.37 & 77.37 & 64.78 \\
\hline 37.00 & 67.08 & 86.46 & 88.82 & 89.04 & 89.13 & 89.12 & 88.94 & 88.60 & 77.47 & 64.58 \\
\hline 38.00 & 66.85 & 85.73 & 88.01 & 88.23 & 88.32 & 88.31 & 88.13 & 87.78 & 76.97 & 63.97 \\
\hline 39.00 & 66.88 & 85.92 & 88.22 & 88.45 & 88.54 & 88.53 & 88.34 & 88.00 & 77.14 & 63.82 \\
\hline 40.00 & 66.52 & 84.75 & 86.92 & 87.14 & 87.23 & 87.23 & 87.04 & 86.71 & 76.44 & 63.63 \\
\hline 41.00 & 66.50 & 84.92 & 87.11 & 87.33 & 87.42 & 87.41 & 87.22 & 86.88 & 76.60 & 63.69 \\
\hline 42.00 & 65.98 & 82.85 & 84.85 & 85.06 & 85.15 & 85.14 & 84.96 & 84.64 & 75.27 & 63.19 \\
\hline 43.00 & 65.92 & 82.99 & 84.99 & 85.20 & 85.28 & 85.28 & 85.09 & 84.77 & 75.39 & 63.31 \\
\hline 44.00 & 62.83 & 64.70 & 65.10 & 65.30 & 65.39 & 65.38 & 65.18 & 64.88 & 64.23 & 61.57 \\
\hline 45.00 & 62.21 & 64.34 & 64.64 & 64.83 & 64.92 & 64.91 & 64.71 & 64.46 & 63.99 & 61.22 \\
\hline
\end{tabular}


Table B - 36. Temperature $\left({ }^{\circ} \mathrm{C}\right)$ map of the west side of plate 1B5 at BOC 143B (100.9 EFPD cumulative).

\begin{tabular}{|c|c|c|c|c|c|c|c|c|c|c|}
\hline \multirow{2}{*}{$\begin{array}{c}\text { Length of } \\
\text { Plate (in) }\end{array}$} & \multicolumn{10}{|c|}{ Width of Plate (inches) } \\
\hline & 0.05 & 0.25 & 0.50 & 0.75 & 1.00 & 1.25 & 1.50 & 1.75 & 2.00 & 2.24 \\
\hline 22.625 & 59.91 & 57.00 & 56.99 & 57.07 & 57.10 & 57.09 & 57.01 & 56.90 & 57.09 & 59.46 \\
\hline 23.00 & 61.09 & 60.03 & 60.63 & 60.72 & 60.76 & 60.75 & 60.67 & 60.44 & 59.02 & 58.83 \\
\hline 24.00 & 64.63 & 83.36 & 85.74 & 85.84 & 85.86 & 85.85 & 85.80 & 85.59 & 73.77 & 61.13 \\
\hline 25.00 & 64.83 & 83.56 & 85.95 & 86.05 & 86.07 & 86.07 & 86.01 & 85.80 & 74.00 & 61.87 \\
\hline 26.00 & 64.96 & 83.61 & 85.99 & 86.09 & 86.11 & 86.11 & 86.04 & 85.83 & 74.20 & 63.29 \\
\hline 27.00 & 65.08 & 83.78 & 86.16 & 86.25 & 86.28 & 86.27 & 86.21 & 86.00 & 74.47 & 63.72 \\
\hline 28.00 & 65.12 & 83.39 & 85.73 & 85.83 & 85.85 & 85.85 & 85.78 & 85.57 & 74.19 & 63.28 \\
\hline 29.00 & 65.25 & 83.57 & 85.91 & 86.01 & 86.03 & 86.02 & 85.96 & 85.74 & 74.28 & 62.37 \\
\hline 30.00 & 65.32 & 83.60 & 85.94 & 86.04 & 86.06 & 86.05 & 85.98 & 85.77 & 74.35 & 62.00 \\
\hline 31.00 & 65.40 & 83.76 & 86.09 & 86.19 & 86.22 & 86.21 & 86.14 & 85.92 & 74.53 & 62.11 \\
\hline 32.00 & 65.37 & 83.45 & 85.75 & 85.85 & 85.88 & 85.87 & 85.79 & 85.58 & 74.38 & 61.94 \\
\hline 33.00 & 65.43 & 83.61 & 85.92 & 86.02 & 86.05 & 86.04 & 85.97 & 85.74 & 74.52 & 62.07 \\
\hline 34.00 & 65.40 & 83.54 & 85.83 & 85.94 & 85.97 & 85.96 & 85.88 & 85.65 & 74.52 & 62.59 \\
\hline 35.00 & 65.43 & 83.68 & 85.98 & 86.09 & 86.12 & 86.11 & 86.03 & 85.80 & 74.76 & 63.65 \\
\hline 36.00 & 65.19 & 82.45 & 84.64 & 84.75 & 84.78 & 84.77 & 84.69 & 84.46 & 74.09 & 64.02 \\
\hline 37.00 & 65.18 & 82.58 & 84.78 & 84.89 & 84.92 & 84.91 & 84.82 & 84.59 & 74.12 & 63.56 \\
\hline 38.00 & 64.98 & 81.74 & 83.86 & 83.97 & 84.00 & 83.99 & 83.90 & 83.67 & 73.53 & 62.42 \\
\hline 39.00 & 64.94 & 81.86 & 83.99 & 84.09 & 84.13 & 84.11 & 84.03 & 83.79 & 73.63 & 61.98 \\
\hline 40.00 & 64.64 & 80.58 & 82.60 & 82.71 & 82.74 & 82.72 & 82.63 & 82.41 & 72.85 & 61.82 \\
\hline 41.00 & 64.56 & 80.68 & 82.70 & 82.81 & 82.85 & 82.83 & 82.74 & 82.51 & 72.95 & 61.95 \\
\hline 42.00 & 64.08 & 78.48 & 80.32 & 80.43 & 80.46 & 80.45 & 80.36 & 80.13 & 71.53 & 61.44 \\
\hline 43.00 & 63.96 & 78.56 & 80.41 & 80.51 & 80.54 & 80.53 & 80.44 & 80.21 & 71.61 & 61.80 \\
\hline 44.00 & 61.34 & 61.31 & 61.60 & 61.72 & 61.77 & 61.75 & 61.63 & 61.42 & 60.90 & 60.79 \\
\hline 45.00 & 60.80 & 60.95 & 61.14 & 61.25 & 61.29 & 61.27 & 61.15 & 60.99 & 60.64 & 60.55 \\
\hline
\end{tabular}


Table B - 37. Temperature $\left({ }^{\circ} \mathrm{C}\right)$ map of the east side of plate $1 \mathrm{~T} 2$ at MOC 143B (118.9 EFPD cumulative).

\begin{tabular}{|c|c|c|c|c|c|c|c|c|c|c|}
\hline \multirow{2}{*}{$\begin{array}{c}\text { Length of } \\
\text { Plate (in) }\end{array}$} & \multicolumn{10}{|c|}{ Width of Plate (inches) } \\
\hline & 0.05 & 0.25 & 0.50 & 0.75 & 1.00 & 1.25 & 1.50 & 1.75 & 2.00 & 2.24 \\
\hline 0.125 & 52.09 & 51.80 & 51.77 & 51.77 & 51.77 & 51.77 & 51.77 & 51.77 & 51.85 & 52.13 \\
\hline 1.00 & 53.40 & 52.29 & 52.35 & 52.36 & 52.36 & 52.36 & 52.36 & 52.32 & 52.35 & 53.28 \\
\hline 2.00 & 57.14 & 71.46 & 73.30 & 73.34 & 73.34 & 73.34 & 73.33 & 73.21 & 64.22 & 55.66 \\
\hline 3.00 & 57.58 & 71.70 & 73.54 & 73.59 & 73.59 & 73.59 & 73.58 & 73.45 & 64.49 & 55.95 \\
\hline 4.00 & 58.50 & 74.41 & 76.46 & 76.51 & 76.51 & 76.51 & 76.50 & 76.36 & 66.33 & 56.71 \\
\hline 5.00 & 58.85 & 74.64 & 76.68 & 76.73 & 76.74 & 76.74 & 76.72 & 76.57 & 66.57 & 56.95 \\
\hline 6.00 & 59.68 & 76.72 & 78.91 & 78.98 & 78.99 & 78.99 & 78.96 & 78.80 & 68.01 & 57.54 \\
\hline 7.00 & 60.08 & 77.00 & 79.19 & 79.25 & 79.27 & 79.27 & 79.24 & 79.07 & 68.29 & 57.82 \\
\hline 8.00 & 60.87 & 78.61 & 80.90 & 80.98 & 80.99 & 80.99 & 80.96 & 80.78 & 69.46 & 58.42 \\
\hline 9.00 & 61.21 & 78.87 & 81.16 & 81.24 & 81.26 & 81.26 & 81.22 & 81.04 & 69.91 & 59.51 \\
\hline 10.00 & 61.73 & 79.25 & 81.54 & 81.63 & 81.65 & 81.65 & 81.61 & 81.42 & 70.20 & 59.75 \\
\hline 11.00 & 62.07 & 79.52 & 81.82 & 81.91 & 81.94 & 81.93 & 81.88 & 81.69 & 70.40 & 59.64 \\
\hline 12.00 & 62.79 & 80.71 & 83.07 & 83.17 & 83.20 & 83.20 & 83.14 & 82.93 & 71.32 & 60.03 \\
\hline 13.00 & 63.13 & 81.01 & 83.37 & 83.47 & 83.50 & 83.50 & 83.44 & 83.23 & 71.64 & 60.38 \\
\hline 14.00 & 63.57 & 81.40 & 83.76 & 83.87 & 83.91 & 83.91 & 83.84 & 83.62 & 72.02 & 60.82 \\
\hline 15.00 & 63.90 & 81.71 & 84.07 & 84.19 & 84.22 & 84.22 & 84.14 & 83.92 & 72.31 & 61.03 \\
\hline 16.00 & 64.34 & 82.22 & 84.60 & 84.72 & 84.76 & 84.76 & 84.68 & 84.44 & 72.78 & 61.28 \\
\hline 17.00 & 64.65 & 82.54 & 84.91 & 85.04 & 85.09 & 85.08 & 85.00 & 84.76 & 73.21 & 62.29 \\
\hline 18.00 & 65.07 & 82.99 & 85.38 & 85.52 & 85.56 & 85.56 & 85.47 & 85.22 & 73.73 & 62.92 \\
\hline 19.00 & 65.40 & 83.32 & 85.70 & 85.85 & 85.90 & 85.89 & 85.79 & 85.54 & 73.97 & 63.04 \\
\hline 20.00 & 65.74 & 83.46 & 85.83 & 85.98 & 86.03 & 86.03 & 85.92 & 85.66 & 74.09 & 62.78 \\
\hline 21.00 & 65.99 & 83.76 & 86.13 & 86.29 & 86.34 & 86.34 & 86.22 & 85.96 & 74.38 & 62.78 \\
\hline 22.00 & 61.45 & 61.50 & 62.13 & 62.28 & 62.34 & 62.33 & 62.20 & 61.92 & 60.53 & 59.78 \\
\hline 22.50 & 59.80 & 58.76 & 58.83 & 58.96 & 59.02 & 59.01 & 58.88 & 58.72 & 58.85 & 59.41 \\
\hline
\end{tabular}


Table B - 38. Temperature $\left({ }^{\circ} \mathrm{C}\right)$ map of the west side of plate 1T2 at MOC 143B (118.9 EFPD cumulative).

\begin{tabular}{|c|c|c|c|c|c|c|c|c|c|c|}
\hline \multirow{2}{*}{$\begin{array}{c}\text { Length of } \\
\text { Plate (in) }\end{array}$} & \multicolumn{10}{|c|}{ Width of Plate (inches) } \\
\hline & 0.05 & 0.25 & 0.50 & 0.75 & 1.00 & 1.25 & 1.50 & 1.75 & 2.00 & 2.24 \\
\hline 0.125 & 52.34 & 51.80 & 51.77 & 51.77 & 51.77 & 51.77 & 51.77 & 51.77 & 51.85 & 52.33 \\
\hline 1.00 & 53.72 & 52.29 & 52.34 & 52.35 & 52.35 & 52.35 & 52.35 & 52.31 & 52.34 & 53.51 \\
\hline 2.00 & 56.75 & 71.43 & 73.27 & 73.31 & 73.31 & 73.31 & 73.30 & 73.18 & 64.20 & 55.15 \\
\hline 3.00 & 57.19 & 71.63 & 73.47 & 73.51 & 73.51 & 73.51 & 73.50 & 73.37 & 64.43 & 55.24 \\
\hline 4.00 & 57.94 & 74.28 & 76.32 & 76.37 & 76.37 & 76.37 & 76.36 & 76.22 & 66.23 & 55.89 \\
\hline 5.00 & 58.30 & 74.44 & 76.47 & 76.52 & 76.53 & 76.53 & 76.52 & 76.37 & 66.40 & 56.05 \\
\hline 6.00 & 58.97 & 76.45 & 78.63 & 78.68 & 78.69 & 78.69 & 78.68 & 78.52 & 67.78 & 56.53 \\
\hline 7.00 & 59.36 & 76.63 & 78.80 & 78.86 & 78.87 & 78.87 & 78.85 & 78.69 & 67.98 & 56.81 \\
\hline 8.00 & 59.98 & 78.15 & 80.43 & 80.49 & 80.50 & 80.49 & 80.48 & 80.31 & 69.07 & 57.26 \\
\hline 9.00 & 60.31 & 78.31 & 80.58 & 80.65 & 80.66 & 80.65 & 80.63 & 80.46 & 69.43 & 59.18 \\
\hline 10.00 & 60.71 & 78.59 & 80.86 & 80.92 & 80.93 & 80.93 & 80.91 & 80.73 & 69.64 & 59.14 \\
\hline 11.00 & 61.03 & 78.75 & 81.01 & 81.08 & 81.09 & 81.09 & 81.06 & 80.89 & 69.75 & 58.55 \\
\hline 12.00 & 61.59 & 79.81 & 82.14 & 82.21 & 82.22 & 82.22 & 82.19 & 82.01 & 70.55 & 58.50 \\
\hline 13.00 & 61.90 & 79.98 & 82.30 & 82.37 & 82.39 & 82.39 & 82.35 & 82.17 & 70.77 & 58.78 \\
\hline 14.00 & 62.25 & 80.25 & 82.57 & 82.64 & 82.66 & 82.65 & 82.62 & 82.43 & 71.04 & 59.25 \\
\hline 15.00 & 62.55 & 80.42 & 82.73 & 82.81 & 82.83 & 82.83 & 82.79 & 82.60 & 71.22 & 59.48 \\
\hline 16.00 & 62.91 & 80.79 & 83.11 & 83.19 & 83.21 & 83.20 & 83.16 & 82.97 & 71.55 & 59.66 \\
\hline 17.00 & 63.18 & 80.97 & 83.28 & 83.36 & 83.38 & 83.38 & 83.33 & 83.14 & 71.87 & 61.43 \\
\hline 18.00 & 63.50 & 81.29 & 83.60 & 83.69 & 83.71 & 83.70 & 83.66 & 83.46 & 72.27 & 62.23 \\
\hline 19.00 & 63.77 & 81.47 & 83.78 & 83.86 & 83.88 & 83.88 & 83.83 & 83.63 & 72.38 & 62.21 \\
\hline 20.00 & 64.02 & 81.44 & 83.73 & 83.82 & 83.84 & 83.84 & 83.78 & 83.58 & 72.36 & 61.22 \\
\hline 21.00 & 64.22 & 81.60 & 83.88 & 83.97 & 84.00 & 83.99 & 83.94 & 83.73 & 72.53 & 60.84 \\
\hline 22.00 & 60.98 & 59.75 & 60.30 & 60.40 & 60.44 & 60.43 & 60.34 & 60.12 & 58.81 & 58.74 \\
\hline 22.50 & 59.90 & 56.94 & 56.92 & 57.00 & 57.04 & 57.03 & 56.94 & 56.84 & 57.06 & 59.97 \\
\hline
\end{tabular}


Table B - 39. Temperature $\left({ }^{\circ} \mathrm{C}\right)$ map of the east side of plate 1B5 at MOC 143B (118.9 EFPD cumulative).

\begin{tabular}{|c|c|c|c|c|c|c|c|c|c|c|}
\hline \multirow{2}{*}{$\begin{array}{l}\text { Length of } \\
\text { Plate (in) }\end{array}$} & \multicolumn{10}{|c|}{ Width of Plate (inches) } \\
\hline 22.625 & 59.89 & 58.80 & 58.88 & 59.01 & 59.07 & 59.07 & 58.93 & 58.77 & 58.87 & 59.26 \\
\hline 23.00 & 61.30 & 61.60 & 62.23 & 62.38 & 62.44 & 62.43 & 62.30 & 62.02 & 60.63 & 59.68 \\
\hline 24.00 & 65.59 & 83.89 & 86.24 & 86.40 & 86.46 & 86.46 & 86.33 & 86.07 & 74.61 & 62.54 \\
\hline 25.00 & 65.84 & 84.23 & 86.58 & 86.75 & 86.81 & 86.80 & 86.68 & 86.40 & 74.94 & 62.95 \\
\hline 26.00 & 66.03 & 84.75 & 87.13 & 87.30 & 87.36 & 87.35 & 87.22 & 86.94 & 75.47 & 63.70 \\
\hline 27.00 & 66.21 & 85.01 & 87.39 & 87.56 & 87.63 & 87.62 & 87.49 & 87.21 & 75.83 & 64.04 \\
\hline 28.00 & 66.37 & 85.20 & 87.58 & 87.76 & 87.82 & 87.82 & 87.68 & 87.39 & 75.94 & 63.92 \\
\hline 29.00 & 66.55 & 85.49 & 87.87 & 88.05 & 88.12 & 88.11 & 87.97 & 87.68 & 76.12 & 63.61 \\
\hline 30.00 & 66.67 & 85.78 & 88.17 & 88.35 & 88.42 & 88.41 & 88.27 & 87.98 & 76.38 & 63.55 \\
\hline 31.00 & 66.80 & 86.03 & 88.42 & 88.61 & 88.68 & 88.67 & 88.52 & 88.23 & 76.64 & 63.73 \\
\hline 32.00 & 66.85 & 86.15 & 88.53 & 88.72 & 88.79 & 88.79 & 88.64 & 88.34 & 76.79 & 63.71 \\
\hline 33.00 & 66.97 & 86.39 & 88.78 & 88.97 & 89.04 & 89.03 & 88.88 & 88.58 & 77.00 & 63.75 \\
\hline 34.00 & 66.88 & 86.09 & 88.44 & 88.63 & 88.70 & 88.70 & 88.54 & 88.24 & 76.88 & 63.92 \\
\hline 35.00 & 66.98 & 86.31 & 88.67 & 88.88 & 88.96 & 88.95 & 88.78 & 88.47 & 77.19 & 64.46 \\
\hline 36.00 & 66.96 & 86.55 & 88.93 & 89.14 & 89.22 & 89.21 & 89.04 & 88.71 & 77.49 & 64.72 \\
\hline 37.00 & 67.03 & 86.77 & 89.17 & 89.38 & 89.46 & 89.46 & 89.28 & 88.95 & 77.60 & 64.53 \\
\hline 38.00 & 66.86 & 86.40 & 88.77 & 88.98 & 89.07 & 89.06 & 88.88 & 88.54 & 77.34 & 63.95 \\
\hline 39.00 & 66.90 & 86.61 & 88.99 & 89.20 & 89.29 & 89.28 & 89.10 & 88.76 & 77.52 & 63.81 \\
\hline 40.00 & 66.58 & 85.62 & 87.89 & 88.11 & 88.20 & 88.19 & 88.01 & 87.67 & 76.94 & 63.66 \\
\hline 41.00 & 66.57 & 85.80 & 88.09 & 88.31 & 88.39 & 88.39 & 88.20 & 87.86 & 77.11 & 63.72 \\
\hline 42.00 & 66.09 & 83.92 & 86.01 & 86.22 & 86.30 & 86.29 & 86.12 & 85.80 & 75.90 & 63.25 \\
\hline 43.00 & 66.04 & 84.06 & 86.16 & 86.37 & 86.45 & 86.45 & 86.26 & 85.94 & 76.03 & 63.37 \\
\hline 44.00 & 62.74 & 64.54 & 64.95 & 65.14 & 65.23 & 65.22 & 65.02 & 64.73 & 64.07 & 61.50 \\
\hline 45.00 & 62.09 & 64.16 & 64.46 & 64.64 & 64.72 & 64.71 & 64.52 & 64.28 & 63.82 & 61.12 \\
\hline
\end{tabular}


Table B - 40. Temperature $\left({ }^{\circ} \mathrm{C}\right)$ map of the west side of plate 1B5 at MOC 143B (118.9 EFPD cumulative).

\begin{tabular}{|c|c|c|c|c|c|c|c|c|c|c|}
\hline \multirow{2}{*}{$\begin{array}{c}\text { Length of } \\
\text { Plate (in) }\end{array}$} & \multicolumn{10}{|c|}{ Width of Plate (inches) } \\
\hline & 0.05 & 0.25 & 0.50 & 0.75 & 1.00 & 1.25 & 1.50 & 1.75 & 2.00 & 2.24 \\
\hline 22.625 & 59.89 & 56.98 & 56.97 & 57.04 & 57.08 & 57.07 & 56.99 & 56.88 & 57.07 & 59.44 \\
\hline 23.00 & 60.97 & 59.79 & 60.33 & 60.43 & 60.46 & 60.45 & 60.37 & 60.15 & 58.85 & 58.74 \\
\hline 24.00 & 64.25 & 81.45 & 83.69 & 83.78 & 83.80 & 83.80 & 83.74 & 83.54 & 72.51 & 60.88 \\
\hline 25.00 & 64.46 & 81.68 & 83.93 & 84.02 & 84.04 & 84.04 & 83.98 & 83.77 & 72.74 & 61.62 \\
\hline 26.00 & 64.63 & 82.11 & 84.38 & 84.47 & 84.50 & 84.49 & 84.43 & 84.22 & 73.19 & 63.03 \\
\hline 27.00 & 64.76 & 82.27 & 84.54 & 84.64 & 84.66 & 84.65 & 84.59 & 84.38 & 73.46 & 63.46 \\
\hline 28.00 & 64.88 & 82.37 & 84.63 & 84.73 & 84.75 & 84.74 & 84.68 & 84.47 & 73.49 & 63.08 \\
\hline 29.00 & 65.00 & 82.54 & 84.81 & 84.90 & 84.93 & 84.92 & 84.85 & 84.64 & 73.58 & 62.18 \\
\hline 30.00 & 65.08 & 82.73 & 85.00 & 85.10 & 85.12 & 85.12 & 85.05 & 84.83 & 73.75 & 61.83 \\
\hline 31.00 & 65.16 & 82.88 & 85.15 & 85.25 & 85.28 & 85.27 & 85.20 & 84.98 & 73.92 & 61.94 \\
\hline 32.00 & 65.19 & 82.90 & 85.16 & 85.26 & 85.29 & 85.28 & 85.20 & 84.99 & 73.98 & 61.80 \\
\hline 33.00 & 65.25 & 83.06 & 85.32 & 85.42 & 85.45 & 85.44 & 85.37 & 85.15 & 74.12 & 61.93 \\
\hline 34.00 & 65.17 & 82.63 & 84.85 & 84.95 & 84.98 & 84.97 & 84.89 & 84.67 & 73.89 & 62.42 \\
\hline 35.00 & 65.19 & 82.76 & 84.99 & 85.09 & 85.12 & 85.11 & 85.03 & 84.81 & 74.12 & 63.47 \\
\hline 36.00 & 65.17 & 82.91 & 85.14 & 85.25 & 85.28 & 85.26 & 85.18 & 84.96 & 74.34 & 63.98 \\
\hline 37.00 & 65.18 & 83.04 & 85.28 & 85.39 & 85.42 & 85.41 & 85.32 & 85.10 & 74.38 & 63.53 \\
\hline 38.00 & 65.03 & 82.58 & 84.77 & 84.88 & 84.91 & 84.90 & 84.82 & 84.59 & 74.03 & 62.42 \\
\hline 39.00 & 64.99 & 82.70 & 84.90 & 85.01 & 85.04 & 85.03 & 84.94 & 84.71 & 74.14 & 61.99 \\
\hline 40.00 & 64.73 & 81.62 & 83.73 & 83.84 & 83.87 & 83.86 & 83.77 & 83.54 & 73.48 & 61.85 \\
\hline 41.00 & 64.66 & 81.72 & 83.84 & 83.95 & 83.98 & 83.97 & 83.88 & 83.65 & 73.59 & 61.99 \\
\hline 42.00 & 64.22 & 79.71 & 81.66 & 81.77 & 81.80 & 81.78 & 81.70 & 81.47 & 72.29 & 61.50 \\
\hline 43.00 & 64.09 & 79.80 & 81.75 & 81.86 & 81.89 & 81.87 & 81.78 & 81.56 & 72.37 & 61.86 \\
\hline 44.00 & 61.30 & 61.26 & 61.55 & 61.67 & 61.72 & 61.70 & 61.58 & 61.36 & 60.84 & 60.75 \\
\hline 45.00 & 60.73 & 60.87 & 61.05 & 61.16 & 61.21 & 61.19 & 61.07 & 60.90 & 60.57 & 60.48 \\
\hline
\end{tabular}


Table B - 41. Temperature $\left({ }^{\circ} \mathrm{C}\right)$ map of the east side of plate 1T2 at MOC 143B (138.9 EFPD cumulative).

\begin{tabular}{|c|c|c|c|c|c|c|c|c|c|c|}
\hline \multirow{2}{*}{$\begin{array}{c}\text { Length of } \\
\text { Plate (in) }\end{array}$} & \multicolumn{10}{|c|}{ Width of Plate (inches) } \\
\hline & 0.05 & 0.25 & 0.50 & 0.75 & 1.00 & 1.25 & 1.50 & 1.75 & 2.00 & 2.24 \\
\hline 0.125 & 52.09 & 51.80 & 51.77 & 51.77 & 51.77 & 51.77 & 51.77 & 51.77 & 51.85 & 52.13 \\
\hline 1.00 & 53.44 & 52.31 & 52.37 & 52.38 & 52.38 & 52.38 & 52.38 & 52.34 & 52.36 & 53.31 \\
\hline 2.00 & 57.35 & 72.13 & 74.02 & 74.06 & 74.07 & 74.07 & 74.06 & 73.93 & 64.66 & 55.81 \\
\hline 3.00 & 57.80 & 72.38 & 74.28 & 74.32 & 74.33 & 74.33 & 74.32 & 74.18 & 64.94 & 56.10 \\
\hline 4.00 & 58.72 & 75.27 & 77.38 & 77.43 & 77.44 & 77.44 & 77.43 & 77.28 & 66.90 & 56.86 \\
\hline 5.00 & 59.06 & 75.49 & 77.61 & 77.66 & 77.67 & 77.67 & 77.65 & 77.50 & 67.13 & 57.10 \\
\hline 6.00 & 59.78 & 76.77 & 78.97 & 79.03 & 79.04 & 79.04 & 79.02 & 78.85 & 68.06 & 57.61 \\
\hline 7.00 & 60.12 & 77.02 & 79.21 & 79.27 & 79.29 & 79.29 & 79.26 & 79.09 & 68.32 & 57.87 \\
\hline 8.00 & 60.87 & 78.42 & 80.69 & 80.77 & 80.79 & 80.79 & 80.75 & 80.57 & 69.35 & 58.42 \\
\hline 9.00 & 61.20 & 78.68 & 80.95 & 81.04 & 81.06 & 81.05 & 81.02 & 80.83 & 69.80 & 59.51 \\
\hline 10.00 & 61.67 & 78.69 & 80.94 & 81.02 & 81.04 & 81.04 & 81.00 & 80.81 & 69.85 & 59.71 \\
\hline 11.00 & 62.00 & 78.96 & 81.21 & 81.30 & 81.32 & 81.32 & 81.27 & 81.07 & 70.05 & 59.60 \\
\hline 12.00 & 62.59 & 79.71 & 81.98 & 82.08 & 82.11 & 82.11 & 82.05 & 81.85 & 70.67 & 59.90 \\
\hline 13.00 & 62.91 & 79.99 & 82.27 & 82.38 & 82.41 & 82.41 & 82.34 & 82.13 & 70.98 & 60.24 \\
\hline 14.00 & 63.30 & 80.11 & 82.37 & 82.48 & 82.51 & 82.51 & 82.44 & 82.22 & 71.18 & 60.64 \\
\hline 15.00 & 63.62 & 80.40 & 82.66 & 82.78 & 82.82 & 82.81 & 82.74 & 82.51 & 71.46 & 60.84 \\
\hline 16.00 & 63.97 & 80.40 & 82.63 & 82.75 & 82.80 & 82.79 & 82.71 & 82.48 & 71.58 & 61.04 \\
\hline 17.00 & 64.27 & 80.70 & 82.93 & 83.06 & 83.11 & 83.10 & 83.01 & 82.78 & 72.00 & 62.03 \\
\hline 18.00 & 64.55 & 80.69 & 82.89 & 83.03 & 83.08 & 83.07 & 82.98 & 82.74 & 72.21 & 62.54 \\
\hline 19.00 & 64.83 & 80.99 & 83.20 & 83.34 & 83.39 & 83.38 & 83.28 & 83.04 & 72.42 & 62.63 \\
\hline 20.00 & 65.22 & 81.46 & 83.67 & 83.81 & 83.87 & 83.86 & 83.76 & 83.50 & 72.75 & 62.42 \\
\hline 21.00 & 65.47 & 81.75 & 83.96 & 84.11 & 84.16 & 84.16 & 84.05 & 83.79 & 73.03 & 62.42 \\
\hline 22.00 & 61.24 & 61.13 & 61.70 & 61.84 & 61.90 & 61.90 & 61.77 & 61.51 & 60.24 & 59.62 \\
\hline 22.50 & 59.69 & 58.61 & 58.66 & 58.79 & 58.85 & 58.85 & 58.71 & 58.56 & 58.72 & 59.32 \\
\hline
\end{tabular}


Table B - 42. Temperature $\left({ }^{\circ} \mathrm{C}\right)$ map of the west side of plate 1T2 at MOC 143B (138.9 EFPD cumulative).

\begin{tabular}{|c|c|c|c|c|c|c|c|c|c|c|}
\hline \multirow{2}{*}{$\begin{array}{c}\text { Length of } \\
\text { Plate (in) }\end{array}$} & \multicolumn{10}{|c|}{ Width of Plate (inches) } \\
\hline & 0.05 & 0.25 & 0.50 & 0.75 & 1.00 & 1.25 & 1.50 & 1.75 & 2.00 & 2.24 \\
\hline 0.125 & 52.34 & 51.80 & 51.77 & 51.77 & 51.77 & 51.77 & 51.77 & 51.77 & 51.85 & 52.33 \\
\hline 1.00 & 53.74 & 52.31 & 52.36 & 52.37 & 52.37 & 52.37 & 52.37 & 52.33 & 52.36 & 53.53 \\
\hline 2.00 & 56.89 & 72.10 & 73.99 & 74.03 & 74.03 & 74.03 & 74.03 & 73.90 & 64.64 & 55.25 \\
\hline 3.00 & 57.34 & 72.30 & 74.20 & 74.24 & 74.24 & 74.24 & 74.23 & 74.10 & 64.87 & 55.35 \\
\hline 4.00 & 58.11 & 75.13 & 77.24 & 77.28 & 77.29 & 77.29 & 77.28 & 77.14 & 66.78 & 56.00 \\
\hline 5.00 & 58.46 & 75.28 & 77.39 & 77.44 & 77.44 & 77.44 & 77.43 & 77.28 & 66.96 & 56.16 \\
\hline 6.00 & 59.02 & 76.48 & 78.67 & 78.72 & 78.73 & 78.72 & 78.71 & 78.56 & 67.82 & 56.57 \\
\hline 7.00 & 59.36 & 76.64 & 78.82 & 78.87 & 78.88 & 78.88 & 78.86 & 78.70 & 68.00 & 56.83 \\
\hline 8.00 & 59.96 & 77.94 & 80.20 & 80.26 & 80.26 & 80.26 & 80.24 & 80.08 & 68.94 & 57.26 \\
\hline 9.00 & 60.29 & 78.10 & 80.35 & 80.41 & 80.42 & 80.42 & 80.40 & 80.23 & 69.30 & 59.18 \\
\hline 10.00 & 60.64 & 78.00 & 80.22 & 80.28 & 80.29 & 80.29 & 80.27 & 80.10 & 69.26 & 59.10 \\
\hline 11.00 & 60.95 & 78.16 & 80.37 & 80.44 & 80.45 & 80.45 & 80.42 & 80.25 & 69.37 & 58.51 \\
\hline 12.00 & 61.42 & 78.78 & 81.02 & 81.08 & 81.10 & 81.10 & 81.07 & 80.89 & 69.88 & 58.40 \\
\hline 13.00 & 61.72 & 78.94 & 81.18 & 81.25 & 81.26 & 81.26 & 81.23 & 81.05 & 70.09 & 58.67 \\
\hline 14.00 & 62.03 & 78.93 & 81.14 & 81.21 & 81.23 & 81.23 & 81.19 & 81.01 & 70.18 & 59.11 \\
\hline 15.00 & 62.31 & 79.10 & 81.30 & 81.38 & 81.39 & 81.39 & 81.35 & 81.17 & 70.35 & 59.33 \\
\hline 16.00 & 62.59 & 78.95 & 81.11 & 81.19 & 81.21 & 81.21 & 81.16 & 80.98 & 70.34 & 59.47 \\
\hline 17.00 & 62.86 & 79.11 & 81.27 & 81.35 & 81.37 & 81.37 & 81.32 & 81.14 & 70.65 & 61.21 \\
\hline 18.00 & 63.08 & 78.96 & 81.09 & 81.17 & 81.19 & 81.19 & 81.14 & 80.95 & 70.73 & 61.92 \\
\hline 19.00 & 63.32 & 79.13 & 81.25 & 81.33 & 81.36 & 81.35 & 81.30 & 81.11 & 70.83 & 61.88 \\
\hline 20.00 & 63.61 & 79.44 & 81.56 & 81.65 & 81.67 & 81.66 & 81.61 & 81.42 & 71.03 & 60.95 \\
\hline 21.00 & 63.81 & 79.59 & 81.71 & 81.80 & 81.82 & 81.82 & 81.76 & 81.57 & 71.19 & 60.58 \\
\hline 22.00 & 60.81 & 59.40 & 59.90 & 60.00 & 60.03 & 60.02 & 59.94 & 59.73 & 58.55 & 58.62 \\
\hline 22.50 & 59.81 & 56.82 & 56.79 & 56.87 & 56.90 & 56.89 & 56.81 & 56.72 & 56.95 & 59.88 \\
\hline
\end{tabular}


Table B - 43. Temperature $\left({ }^{\circ} \mathrm{C}\right)$ map of the east side of plate 1B5 at MOC 143B (138.9 EFPD cumulative).

\begin{tabular}{|c|c|c|c|c|c|c|c|c|c|c|}
\hline \multirow{2}{*}{$\begin{array}{l}\text { Length of } \\
\text { Plate (in) }\end{array}$} & \multicolumn{10}{|c|}{ Width of Plate (inches) } \\
\hline 22.625 & 59.78 & 58.65 & 58.71 & 58.84 & 58.90 & 58.89 & 58.76 & 58.61 & 58.72 & 59.18 \\
\hline 23.00 & 61.09 & 61.22 & 61.79 & 61.94 & 62.00 & 61.99 & 61.86 & 61.60 & 60.34 & 59.52 \\
\hline 24.00 & 65.07 & 81.85 & 84.03 & 84.19 & 84.25 & 84.24 & 84.12 & 83.86 & 73.22 & 62.17 \\
\hline 25.00 & 65.30 & 82.15 & 84.35 & 84.51 & 84.57 & 84.56 & 84.44 & 84.17 & 73.53 & 62.58 \\
\hline 26.00 & 65.49 & 82.58 & 84.79 & 84.95 & 85.02 & 85.01 & 84.88 & 84.62 & 74.00 & 63.32 \\
\hline 27.00 & 65.67 & 82.83 & 85.04 & 85.21 & 85.27 & 85.27 & 85.14 & 84.87 & 74.34 & 63.64 \\
\hline 28.00 & 65.87 & 83.43 & 85.67 & 85.84 & 85.90 & 85.90 & 85.77 & 85.49 & 74.70 & 63.55 \\
\hline 29.00 & 66.03 & 83.70 & 85.95 & 86.12 & 86.18 & 86.18 & 86.04 & 85.76 & 74.87 & 63.25 \\
\hline 30.00 & 66.12 & 83.71 & 85.94 & 86.11 & 86.18 & 86.17 & 86.03 & 85.75 & 74.94 & 63.17 \\
\hline 31.00 & 66.25 & 83.94 & 86.18 & 86.36 & 86.42 & 86.42 & 86.28 & 85.99 & 75.20 & 63.35 \\
\hline 32.00 & 66.34 & 84.36 & 86.62 & 86.80 & 86.87 & 86.86 & 86.72 & 86.43 & 75.53 & 63.36 \\
\hline 33.00 & 66.46 & 84.60 & 86.85 & 87.04 & 87.11 & 87.10 & 86.95 & 86.66 & 75.74 & 63.40 \\
\hline 34.00 & 66.57 & 85.26 & 87.56 & 87.75 & 87.82 & 87.81 & 87.66 & 87.37 & 76.24 & 63.69 \\
\hline 35.00 & 66.66 & 85.49 & 87.79 & 87.98 & 88.05 & 88.04 & 87.89 & 87.59 & 76.55 & 64.22 \\
\hline 36.00 & 66.55 & 85.23 & 87.51 & 87.70 & 87.77 & 87.77 & 87.61 & 87.31 & 76.53 & 64.41 \\
\hline 37.00 & 66.62 & 85.45 & 87.73 & 87.92 & 87.99 & 87.99 & 87.83 & 87.53 & 76.64 & 64.23 \\
\hline 38.00 & 66.58 & 85.69 & 87.98 & 88.18 & 88.26 & 88.25 & 88.08 & 87.78 & 76.77 & 63.75 \\
\hline 39.00 & 66.62 & 85.89 & 88.19 & 88.40 & 88.48 & 88.48 & 88.30 & 87.98 & 76.95 & 63.61 \\
\hline 40.00 & 66.36 & 85.15 & 87.38 & 87.58 & 87.66 & 87.66 & 87.48 & 87.17 & 76.53 & 63.50 \\
\hline 41.00 & 66.36 & 85.33 & 87.57 & 87.78 & 87.86 & 87.86 & 87.68 & 87.36 & 76.70 & 63.56 \\
\hline 42.00 & 65.96 & 84.07 & 86.19 & 86.39 & 86.47 & 86.46 & 86.30 & 85.98 & 75.89 & 63.14 \\
\hline 43.00 & 65.91 & 84.21 & 86.35 & 86.55 & 86.62 & 86.62 & 86.45 & 86.14 & 76.02 & 63.27 \\
\hline 44.00 & 62.52 & 64.19 & 64.59 & 64.77 & 64.85 & 64.84 & 64.66 & 64.37 & 63.74 & 61.33 \\
\hline 45.00 & 61.85 & 63.80 & 64.08 & 64.25 & 64.34 & 64.32 & 64.14 & 63.91 & 63.47 & 60.93 \\
\hline
\end{tabular}


Table B - 44. Temperature $\left({ }^{\circ} \mathrm{C}\right)$ map of the west side of plate 1B5 at MOC 143B (138.9 EFPD cumulative).

\begin{tabular}{|c|c|c|c|c|c|c|c|c|c|c|}
\hline \multirow{2}{*}{$\begin{array}{c}\text { Length of } \\
\text { Plate (in) }\end{array}$} & \multicolumn{10}{|c|}{ Width of Plate (inches) } \\
\hline & 0.05 & 0.25 & 0.50 & 0.75 & 1.00 & 1.25 & 1.50 & 1.75 & 2.00 & 2.24 \\
\hline 22.625 & 59.80 & 56.86 & 56.83 & 56.91 & 56.94 & 56.93 & 56.85 & 56.76 & 56.96 & 59.36 \\
\hline 23.00 & 60.80 & 59.44 & 59.93 & 60.02 & 60.06 & 60.05 & 59.96 & 59.76 & 58.59 & 58.62 \\
\hline 24.00 & 63.83 & 79.42 & 81.50 & 81.59 & 81.61 & 81.60 & 81.55 & 81.36 & 71.15 & 60.61 \\
\hline 25.00 & 64.03 & 79.63 & 81.72 & 81.81 & 81.83 & 81.83 & 81.77 & 81.57 & 71.37 & 61.33 \\
\hline 26.00 & 64.19 & 79.98 & 82.08 & 82.17 & 82.19 & 82.18 & 82.12 & 81.93 & 71.76 & 62.71 \\
\hline 27.00 & 64.32 & 80.13 & 82.24 & 82.33 & 82.35 & 82.34 & 82.28 & 82.08 & 72.02 & 63.12 \\
\hline 28.00 & 64.47 & 80.64 & 82.77 & 82.87 & 82.89 & 82.88 & 82.82 & 82.62 & 72.31 & 62.78 \\
\hline 29.00 & 64.59 & 80.81 & 82.94 & 83.03 & 83.06 & 83.05 & 82.98 & 82.78 & 72.39 & 61.90 \\
\hline 30.00 & 64.63 & 80.72 & 82.83 & 82.92 & 82.95 & 82.94 & 82.87 & 82.67 & 72.38 & 61.54 \\
\hline 31.00 & 64.71 & 80.86 & 82.98 & 83.07 & 83.10 & 83.09 & 83.02 & 82.82 & 72.55 & 61.65 \\
\hline 32.00 & 64.77 & 81.19 & 83.33 & 83.42 & 83.45 & 83.44 & 83.37 & 83.16 & 72.81 & 61.53 \\
\hline 33.00 & 64.83 & 81.35 & 83.49 & 83.58 & 83.61 & 83.60 & 83.53 & 83.32 & 72.94 & 61.67 \\
\hline 34.00 & 64.91 & 81.91 & 84.08 & 84.18 & 84.20 & 84.19 & 84.12 & 83.91 & 73.35 & 62.23 \\
\hline 35.00 & 64.93 & 82.05 & 84.22 & 84.32 & 84.34 & 84.33 & 84.26 & 84.05 & 73.58 & 63.26 \\
\hline 36.00 & 64.84 & 81.70 & 83.84 & 83.94 & 83.97 & 83.95 & 83.88 & 83.67 & 73.48 & 63.71 \\
\hline 37.00 & 64.83 & 81.83 & 83.97 & 84.07 & 84.10 & 84.09 & 84.01 & 83.80 & 73.51 & 63.27 \\
\hline 38.00 & 64.79 & 81.98 & 84.14 & 84.24 & 84.26 & 84.25 & 84.17 & 83.96 & 73.57 & 62.25 \\
\hline 39.00 & 64.76 & 82.10 & 84.26 & 84.37 & 84.39 & 84.38 & 84.30 & 84.08 & 73.68 & 61.83 \\
\hline 40.00 & 64.53 & 81.27 & 83.36 & 83.46 & 83.49 & 83.48 & 83.40 & 83.18 & 73.18 & 61.72 \\
\hline 41.00 & 64.46 & 81.38 & 83.48 & 83.58 & 83.61 & 83.59 & 83.51 & 83.29 & 73.29 & 61.85 \\
\hline 42.00 & 64.11 & 80.00 & 81.99 & 82.09 & 82.12 & 82.10 & 82.02 & 81.80 & 72.39 & 61.41 \\
\hline 43.00 & 63.99 & 80.09 & 82.08 & 82.18 & 82.21 & 82.20 & 82.11 & 81.89 & 72.48 & 61.78 \\
\hline 44.00 & 61.13 & 61.00 & 61.29 & 61.40 & 61.45 & 61.43 & 61.31 & 61.10 & 60.60 & 60.60 \\
\hline 45.00 & 60.53 & 60.60 & 60.77 & 60.88 & 60.92 & 60.90 & 60.78 & 60.63 & 60.32 & 60.32 \\
\hline
\end{tabular}


Table B - 45. Temperature $\left({ }^{\circ} \mathrm{C}\right)$ map of the east side of plate 1T2 at EOC 143B (158.2 EFPD cumulative).

\begin{tabular}{|c||c|c|c|c|c|c|c|c|c|c|}
\hline \multirow{2}{*}{$\begin{array}{c}\text { Length of } \\
\text { Plate (in) }\end{array}$} & \multicolumn{10}{|c|}{ Width of Plate (inches) } \\
\hline & 0.05 & 0.25 & 0.50 & 0.75 & 1.00 & 1.25 & 1.50 & 1.75 & 2.00 & 2.24 \\
\hline 0.125 & 52.09 & 51.80 & 51.77 & 51.77 & 51.77 & 51.77 & 51.77 & 51.77 & 51.85 & 52.13 \\
\hline 1.00 & 53.41 & 52.32 & 52.38 & 52.39 & 52.39 & 52.39 & 52.39 & 52.35 & 52.36 & 53.28 \\
\hline 2.00 & 57.25 & 72.64 & 74.58 & 74.62 & 74.63 & 74.62 & 74.62 & 74.49 & 64.96 & 55.72 \\
\hline 3.00 & 57.68 & 72.89 & 74.83 & 74.88 & 74.88 & 74.88 & 74.87 & 74.74 & 65.23 & 55.99 \\
\hline 4.00 & 58.56 & 75.93 & 78.10 & 78.16 & 78.16 & 78.16 & 78.15 & 78.00 & 67.29 & 56.71 \\
\hline 5.00 & 58.89 & 76.14 & 78.32 & 78.37 & 78.38 & 78.38 & 78.37 & 78.21 & 67.51 & 56.94 \\
\hline 6.00 & 59.52 & 77.57 & 79.84 & 79.90 & 79.91 & 79.91 & 79.89 & 79.72 & 68.52 & 57.38 \\
\hline 7.00 & 59.88 & 77.82 & 80.09 & 80.16 & 80.17 & 80.17 & 80.15 & 79.97 & 68.79 & 57.64 \\
\hline 8.00 & 60.52 & 79.43 & 81.81 & 81.88 & 81.90 & 81.89 & 81.86 & 81.68 & 69.93 & 58.11 \\
\hline 9.00 & 60.84 & 79.66 & 82.04 & 82.12 & 82.14 & 82.14 & 82.10 & 81.91 & 70.35 & 59.18 \\
\hline 10.00 & 61.22 & 79.73 & 82.09 & 82.18 & 82.20 & 82.19 & 82.15 & 81.96 & 70.43 & 59.31 \\
\hline 11.00 & 61.52 & 79.97 & 82.33 & 82.42 & 82.44 & 82.44 & 82.40 & 82.20 & 70.60 & 59.18 \\
\hline 12.00 & 62.02 & 80.85 & 83.26 & 83.35 & 83.38 & 83.37 & 83.32 & 83.12 & 71.28 & 59.40 \\
\hline 13.00 & 62.31 & 81.10 & 83.51 & 83.61 & 83.63 & 83.63 & 83.57 & 83.36 & 71.56 & 59.72 \\
\hline 14.00 & 62.63 & 81.15 & 83.53 & 83.63 & 83.66 & 83.66 & 83.60 & 83.38 & 71.70 & 60.06 \\
\hline 15.00 & 62.91 & 81.40 & 83.78 & 83.89 & 83.92 & 83.92 & 83.85 & 83.63 & 71.94 & 60.24 \\
\hline 16.00 & 63.20 & 81.51 & 83.87 & 83.98 & 84.02 & 84.02 & 83.94 & 83.72 & 72.11 & 60.38 \\
\hline 17.00 & 63.46 & 81.76 & 84.12 & 84.24 & 84.28 & 84.28 & 84.20 & 83.97 & 72.49 & 61.34 \\
\hline 18.00 & 63.67 & 81.69 & 84.02 & 84.14 & 84.18 & 84.18 & 84.10 & 83.87 & 72.63 & 61.79 \\
\hline 19.00 & 63.92 & 81.94 & 84.27 & 84.40 & 84.44 & 84.44 & 84.35 & 84.11 & 72.80 & 61.86 \\
\hline 20.00 & 64.22 & 82.22 & 84.56 & 84.69 & 84.73 & 84.73 & 84.64 & 84.40 & 72.99 & 61.58 \\
\hline 21.00 & 64.43 & 82.46 & 84.79 & 84.93 & 84.97 & 84.97 & 84.87 & 84.63 & 73.22 & 61.55 \\
\hline 22.00 & 60.55 & 60.63 & 61.23 & 61.36 & 61.41 & 61.40 & 61.29 & 61.04 & 59.68 & 59.07 \\
\hline 22.50 & 59.19 & 57.92 & 57.95 & 58.06 & 58.11 & 58.11 & 58.00 & 57.86 & 58.05 & 58.91 \\
\hline
\end{tabular}


Table B - 46. Temperature $\left({ }^{\circ} \mathrm{C}\right)$ map of the west side of plate 1T2 at EOC 143B (158.2 EFPD cumulative).

\begin{tabular}{|c|c|c|c|c|c|c|c|c|c|c|}
\hline \multirow{2}{*}{$\begin{array}{c}\text { Length of } \\
\text { Plate (in) }\end{array}$} & \multicolumn{10}{|c|}{ Width of Plate (inches) } \\
\hline & 0.05 & 0.25 & 0.50 & 0.75 & 1.00 & 1.25 & 1.50 & 1.75 & 2.00 & 2.24 \\
\hline 0.125 & 52.34 & 51.80 & 51.77 & 51.77 & 51.77 & 51.77 & 51.77 & 51.77 & 51.85 & 52.33 \\
\hline 1.00 & 53.74 & 52.32 & 52.38 & 52.39 & 52.39 & 52.39 & 52.39 & 52.34 & 52.36 & 53.52 \\
\hline 2.00 & 56.89 & 72.61 & 74.55 & 74.59 & 74.60 & 74.60 & 74.59 & 74.46 & 64.94 & 55.21 \\
\hline 3.00 & 57.34 & 72.82 & 74.76 & 74.80 & 74.81 & 74.81 & 74.80 & 74.66 & 65.18 & 55.31 \\
\hline 4.00 & 58.10 & 75.81 & 77.98 & 78.03 & 78.03 & 78.03 & 78.02 & 77.88 & 67.19 & 55.95 \\
\hline 5.00 & 58.45 & 75.96 & 78.13 & 78.18 & 78.19 & 78.19 & 78.18 & 78.02 & 67.36 & 56.09 \\
\hline 6.00 & 58.99 & 77.32 & 79.58 & 79.64 & 79.64 & 79.64 & 79.63 & 79.47 & 68.31 & 56.47 \\
\hline 7.00 & 59.36 & 77.49 & 79.75 & 79.81 & 79.82 & 79.81 & 79.80 & 79.63 & 68.51 & 56.74 \\
\hline 8.00 & 59.92 & 79.02 & 81.39 & 81.45 & 81.46 & 81.46 & 81.44 & 81.27 & 69.59 & 57.13 \\
\hline 9.00 & 60.24 & 79.18 & 81.54 & 81.60 & 81.61 & 81.61 & 81.59 & 81.42 & 69.93 & 59.03 \\
\hline 10.00 & 60.56 & 79.17 & 81.50 & 81.57 & 81.58 & 81.58 & 81.56 & 81.38 & 69.94 & 58.92 \\
\hline 11.00 & 60.86 & 79.32 & 81.66 & 81.72 & 81.74 & 81.73 & 81.71 & 81.53 & 70.04 & 58.32 \\
\hline 12.00 & 61.31 & 80.11 & 82.48 & 82.55 & 82.56 & 82.56 & 82.53 & 82.35 & 70.64 & 58.17 \\
\hline 13.00 & 61.60 & 80.27 & 82.64 & 82.71 & 82.72 & 82.72 & 82.69 & 82.50 & 70.84 & 58.43 \\
\hline 14.00 & 61.87 & 80.22 & 82.56 & 82.64 & 82.65 & 82.65 & 82.61 & 82.43 & 70.90 & 58.84 \\
\hline 15.00 & 62.14 & 80.38 & 82.72 & 82.79 & 82.81 & 82.81 & 82.77 & 82.58 & 71.06 & 59.04 \\
\hline 16.00 & 62.40 & 80.38 & 82.69 & 82.77 & 82.79 & 82.79 & 82.75 & 82.55 & 71.14 & 59.16 \\
\hline 17.00 & 62.65 & 80.54 & 82.85 & 82.93 & 82.95 & 82.94 & 82.90 & 82.71 & 71.43 & 60.86 \\
\hline 18.00 & 62.84 & 80.37 & 82.65 & 82.73 & 82.75 & 82.75 & 82.70 & 82.50 & 71.49 & 61.54 \\
\hline 19.00 & 63.06 & 80.52 & 82.80 & 82.88 & 82.90 & 82.90 & 82.85 & 82.65 & 71.58 & 61.48 \\
\hline 20.00 & 63.31 & 80.70 & 82.97 & 83.06 & 83.08 & 83.08 & 83.03 & 82.83 & 71.68 & 60.52 \\
\hline 21.00 & 63.49 & 80.85 & 83.11 & 83.20 & 83.22 & 83.22 & 83.16 & 82.96 & 71.83 & 60.14 \\
\hline 22.00 & 60.53 & 59.34 & 59.88 & 59.97 & 60.00 & 60.00 & 59.92 & 59.70 & 58.41 & 58.31 \\
\hline 22.50 & 59.57 & 56.58 & 56.56 & 56.62 & 56.66 & 56.65 & 56.57 & 56.48 & 56.72 & 59.61 \\
\hline
\end{tabular}


Table B - 47. Temperature $\left({ }^{\circ} \mathrm{C}\right)$ map of the east side of plate 1B5 at EOC 143B (158.2 EFPD cumulative).

\begin{tabular}{|c||c|c|c|c|c|c|c|c|c|c|}
\hline \multirow{2}{*}{$\begin{array}{l}\text { Length of } \\
\text { Plate (in) }\end{array}$} & 0.05 & 0.25 & 0.50 & 0.75 & 1.00 & 1.25 & 1.50 & 1.75 & 2.00 & 2.24 \\
\hline 22.625 & 59.27 & 57.96 & 58.00 & 58.11 & 58.16 & 58.15 & 58.04 & 57.91 & 58.06 & 58.76 \\
\hline 23.00 & 60.47 & 60.70 & 61.29 & 61.42 & 61.47 & 61.46 & 61.35 & 61.10 & 59.76 & 59.03 \\
\hline 24.00 & 64.22 & 82.53 & 84.83 & 84.97 & 85.02 & 85.02 & 84.92 & 84.67 & 73.39 & 61.47 \\
\hline 25.00 & 64.44 & 82.81 & 85.12 & 85.26 & 85.31 & 85.31 & 85.21 & 84.96 & 73.67 & 61.86 \\
\hline 26.00 & 64.64 & 83.27 & 85.60 & 85.75 & 85.80 & 85.80 & 85.69 & 85.44 & 74.15 & 62.59 \\
\hline 27.00 & 64.81 & 83.49 & 85.82 & 85.97 & 86.02 & 86.02 & 85.91 & 85.65 & 74.46 & 62.91 \\
\hline 28.00 & 65.00 & 84.15 & 86.52 & 86.67 & 86.72 & 86.71 & 86.60 & 86.35 & 74.86 & 62.82 \\
\hline 29.00 & 65.15 & 84.38 & 86.75 & 86.90 & 86.96 & 86.95 & 86.84 & 86.58 & 74.99 & 62.50 \\
\hline 30.00 & 65.22 & 84.22 & 86.57 & 86.72 & 86.78 & 86.77 & 86.66 & 86.39 & 74.95 & 62.41 \\
\hline 31.00 & 65.33 & 84.42 & 86.77 & 86.93 & 86.99 & 86.98 & 86.86 & 86.60 & 75.17 & 62.58 \\
\hline 32.00 & 65.44 & 84.76 & 87.12 & 87.28 & 87.33 & 87.33 & 87.21 & 86.94 & 75.44 & 62.60 \\
\hline 33.00 & 65.55 & 84.98 & 87.35 & 87.51 & 87.57 & 87.56 & 87.44 & 87.17 & 75.63 & 62.65 \\
\hline 34.00 & 65.66 & 85.54 & 87.94 & 88.10 & 88.16 & 88.16 & 88.03 & 87.76 & 76.07 & 62.94 \\
\hline 35.00 & 65.74 & 85.74 & 88.14 & 88.31 & 88.36 & 88.36 & 88.23 & 87.96 & 76.34 & 63.45 \\
\hline 36.00 & 65.66 & 85.32 & 87.68 & 87.85 & 87.91 & 87.90 & 87.77 & 87.49 & 76.22 & 63.67 \\
\hline 37.00 & 65.72 & 85.51 & 87.87 & 88.04 & 88.10 & 88.10 & 87.96 & 87.68 & 76.30 & 63.47 \\
\hline 38.00 & 65.70 & 85.57 & 87.94 & 88.10 & 88.17 & 88.16 & 88.03 & 87.74 & 76.32 & 63.02 \\
\hline 39.00 & 65.73 & 85.75 & 88.12 & 88.29 & 88.35 & 88.35 & 88.21 & 87.93 & 76.47 & 62.88 \\
\hline 40.00 & 65.48 & 84.74 & 87.02 & 87.19 & 87.26 & 87.25 & 87.11 & 86.82 & 75.87 & 62.78 \\
\hline 41.00 & 65.48 & 84.90 & 87.19 & 87.36 & 87.42 & 87.42 & 87.28 & 86.99 & 76.03 & 62.84 \\
\hline 42.00 & 65.10 & 83.45 & 85.61 & 85.79 & 85.85 & 85.85 & 85.70 & 85.42 & 75.10 & 62.44 \\
\hline 43.00 & 65.05 & 83.59 & 85.75 & 85.93 & 85.99 & 85.99 & 85.84 & 85.55 & 75.22 & 62.56 \\
\hline 44.00 & 61.66 & 62.95 & 63.32 & 63.48 & 63.55 & 63.54 & 63.38 & 63.12 & 62.54 & 60.64 \\
\hline 45.00 & 61.00 & 62.54 & 62.78 & 62.93 & 63.00 & 62.99 & 62.83 & 62.63 & 62.25 & 60.25 \\
\hline
\end{tabular}


Table B - 48. Temperature $\left({ }^{\circ} \mathrm{C}\right)$ map of the west side of plate 1B5 at EOC 143B (158.2 EFPD cumulative).

\begin{tabular}{|c|c|c|c|c|c|c|c|c|c|c|}
\hline \multirow{2}{*}{$\begin{array}{c}\text { Length of } \\
\text { Plate (in) }\end{array}$} & \multicolumn{10}{|c|}{ Width of Plate (inches) } \\
\hline & 0.05 & 0.25 & 0.50 & 0.75 & 1.00 & 1.25 & 1.50 & 1.75 & 2.00 & 2.24 \\
\hline 22.625 & 59.55 & 56.62 & 56.59 & 56.66 & 56.70 & 56.69 & 56.61 & 56.52 & 56.73 & 59.09 \\
\hline 23.00 & 60.55 & 59.37 & 59.90 & 59.99 & 60.02 & 60.01 & 59.94 & 59.73 & 58.44 & 58.34 \\
\hline 24.00 & 63.59 & 80.74 & 82.97 & 83.06 & 83.08 & 83.07 & 83.02 & 82.82 & 71.85 & 60.25 \\
\hline 25.00 & 63.79 & 80.96 & 83.19 & 83.28 & 83.31 & 83.30 & 83.24 & 83.04 & 72.07 & 60.96 \\
\hline 26.00 & 63.95 & 81.37 & 83.62 & 83.71 & 83.73 & 83.73 & 83.67 & 83.47 & 72.50 & 62.32 \\
\hline 27.00 & 64.07 & 81.52 & 83.77 & 83.87 & 83.89 & 83.88 & 83.82 & 83.62 & 72.75 & 62.74 \\
\hline 28.00 & 64.24 & 82.12 & 84.41 & 84.50 & 84.53 & 84.52 & 84.46 & 84.25 & 73.10 & 62.39 \\
\hline 29.00 & 64.34 & 82.28 & 84.57 & 84.66 & 84.69 & 84.68 & 84.62 & 84.41 & 73.17 & 61.51 \\
\hline 30.00 & 64.36 & 82.06 & 84.32 & 84.41 & 84.44 & 84.43 & 84.36 & 84.15 & 73.07 & 61.15 \\
\hline 31.00 & 64.43 & 82.20 & 84.46 & 84.55 & 84.58 & 84.57 & 84.50 & 84.29 & 73.23 & 61.24 \\
\hline 32.00 & 64.50 & 82.46 & 84.74 & 84.83 & 84.86 & 84.85 & 84.78 & 84.57 & 73.45 & 61.14 \\
\hline 33.00 & 64.55 & 82.62 & 84.89 & 84.99 & 85.02 & 85.01 & 84.94 & 84.72 & 73.57 & 61.26 \\
\hline 34.00 & 64.63 & 83.12 & 85.43 & 85.52 & 85.55 & 85.54 & 85.47 & 85.25 & 73.95 & 61.82 \\
\hline 35.00 & 64.65 & 83.25 & 85.56 & 85.66 & 85.69 & 85.68 & 85.60 & 85.38 & 74.17 & 62.84 \\
\hline 36.00 & 64.54 & 82.76 & 85.03 & 85.13 & 85.15 & 85.14 & 85.07 & 84.85 & 73.99 & 63.30 \\
\hline 37.00 & 64.54 & 82.89 & 85.16 & 85.26 & 85.28 & 85.27 & 85.20 & 84.98 & 74.02 & 62.85 \\
\hline 38.00 & 64.49 & 82.88 & 85.15 & 85.25 & 85.28 & 85.26 & 85.19 & 84.96 & 73.97 & 61.84 \\
\hline 39.00 & 64.45 & 83.00 & 85.27 & 85.37 & 85.40 & 85.39 & 85.31 & 85.08 & 74.08 & 61.42 \\
\hline 40.00 & 64.21 & 81.91 & 84.09 & 84.19 & 84.22 & 84.21 & 84.13 & 83.90 & 73.42 & 61.30 \\
\hline 41.00 & 64.14 & 82.02 & 84.20 & 84.30 & 84.33 & 84.32 & 84.24 & 84.01 & 73.52 & 61.43 \\
\hline 42.00 & 63.77 & 80.48 & 82.54 & 82.64 & 82.67 & 82.65 & 82.57 & 82.34 & 72.52 & 61.00 \\
\hline 43.00 & 63.65 & 80.56 & 82.63 & 82.73 & 82.76 & 82.74 & 82.66 & 82.43 & 72.61 & 61.35 \\
\hline 44.00 & 60.73 & 60.60 & 60.89 & 61.00 & 61.04 & 61.02 & 60.91 & 60.70 & 60.21 & 60.16 \\
\hline 45.00 & 60.13 & 60.18 & 60.34 & 60.44 & 60.48 & 60.46 & 60.35 & 60.20 & 59.91 & 59.88 \\
\hline
\end{tabular}

